\title{
Supplementary Information of:
}

\section{Can MCDA guide transdisciplinary endeavors? A framework applied to co-developing a flood forecasting system in West Africa}

\author{
Judit Lienert $^{1}$, Jafet Andersson ${ }^{2}$, Daniel Hofmann ${ }^{1}$, Francisco Silva Pinto ${ }^{1}$, Martijn Kuller ${ }^{1}$ \\ ${ }^{1}$ Eawag: Swiss Federal Institute of Aquatic Science \& Technology, Environmental Social Sciences (ESS) Department, Ueber- \\ landstrasse 133, 8600 Duebendorf, Switzerland \\ 2 Swedish Meteorological and Hydrological Institute (SMHI), Hydrology Research, 60176 Norrköping, Sweden
}

Correspondence to: Judit Lienert (judit.lienert@eawag.ch)

This document is the Supplementary Information for the preprint of an article that has been accepted for posting on Hydrology and Earth System Sciences (HESS). This manuscript version is made available under the Creative Commons Attribution 4.0 license https://creativecommons.org/licenses/by/4.0/

\author{
Hydrology and Earth Systems Sciences (HESS) Preprints (2021) \\ https://hess.copernicus.org/preprints/preprints.html
}




\section{Contents}

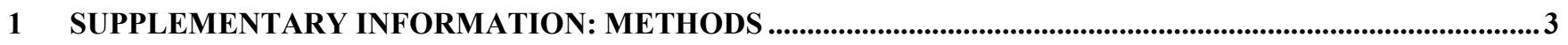

1.1 GENERATING FANFAR SYSTEM CONFIGURATIONS....................................................................................... 3

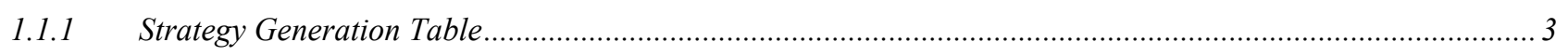

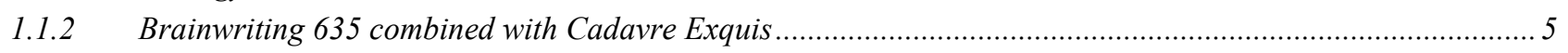

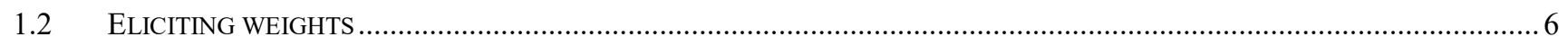

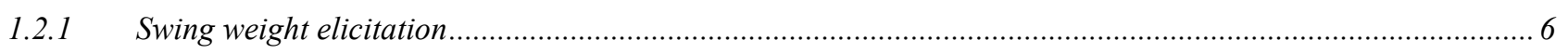

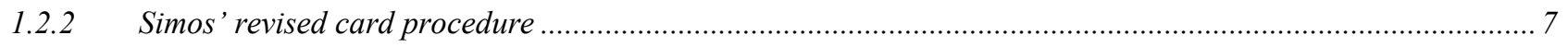

1.2.3 Observations from weight elicitation informing sensitivity analyses.......................................................... 8

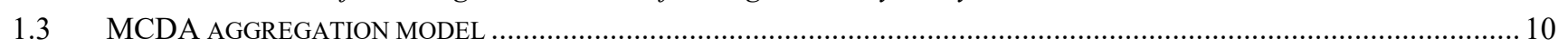

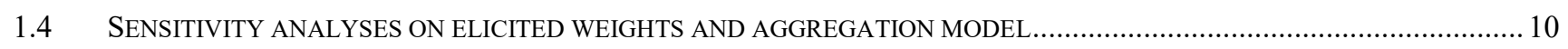

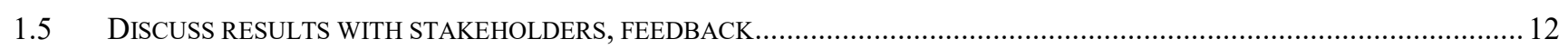

2 SUPPLEMENTARY INFORMATION: RESULTS ……........................................................................................... 14

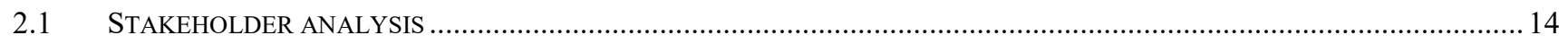

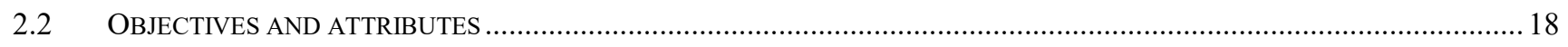

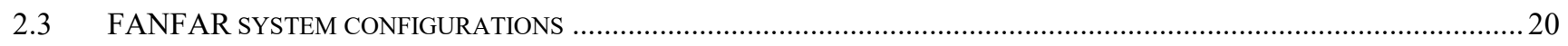

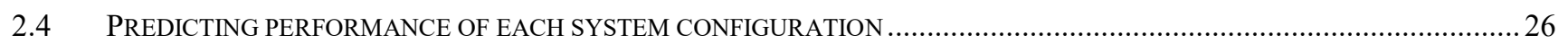

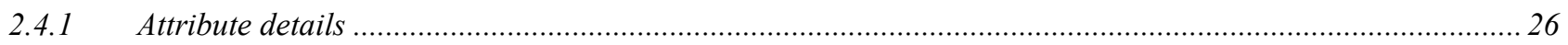

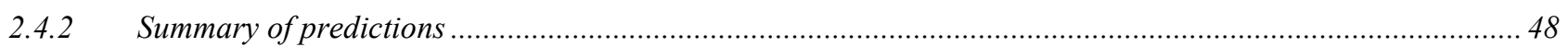

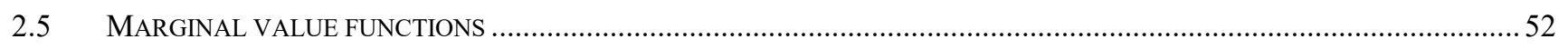

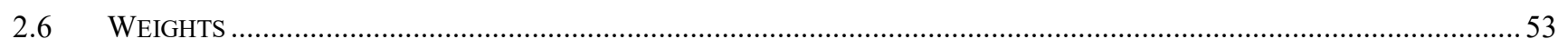

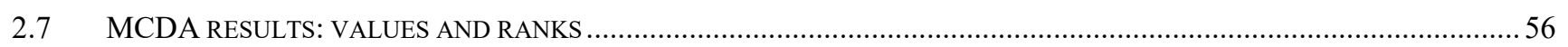

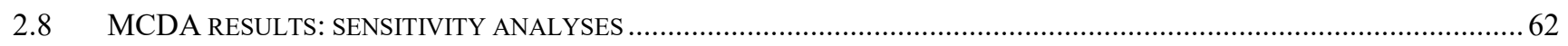

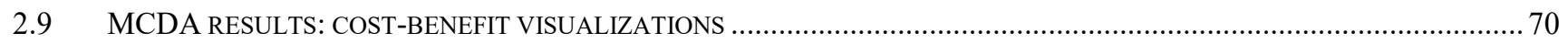

2.10 RESULTS: STAKEHOLDERS’ PERCEIVED SATISFACTION WITH FANFAR SYSTEM .................................................... 74

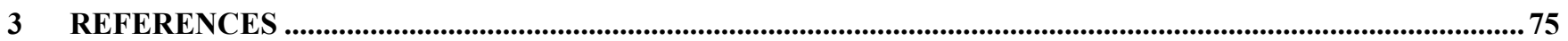




\section{Supplementary Information: Methods}

\subsection{Generating FANFAR system configurations}

The FANFAR system configurations consisted of different possible configurations of the FANFAR flood forecasting and alert system. These were defined using several participatory approaches during the first FANFAR workshop (Niamey, Niger, Sept. 2018). We utilized the Strategy Generation Table (Gregory et al., 2012;Howard, 1988) to co-design possible system configurations for the two FANFAR platforms "Hydrology Thematic Exploitation Platform" (Hydrology-TEP or H-TEP) and the "Interactive Visualization Portal" (IVP) together with the workshop participants. The Hydrology-TEP is the ICT environment in which the forecast production system is deployed to produce new forecasts and alerts every day. In the FANFAR co-design workshops, this is primarily intended for experienced hydrologists and ICT experts. The IVP is the web visualization interface of FANFAR (displaying outputs from H-TEP), which is normally accessed by any user. All emergency managers and most of the participating national hydrologists interacted with the FANFAR system through the IVP in the co-design workshops and in between.

To prepare the workshop, we defined different system elements of the H-TEP and IVP (e.g., forecasted variables, observed variables, distribution channels, etc.). Possible characteristics of these elements were developed by experts of the FANFAR consortium (e.g., possible characteristics for the element "forecasted variables" were river discharge, water level, precipitation, etc., and different combinations). During the "Strategy Generation Table" session, we presented these elements and their possible characteristics to the participants. We pre-defined three strategies, for which we asked the participants to discuss and decide on suitable characteristics for each system element, one by one: 1) "The most easy-to-use system", 2) "The most attractive system for West Africa", and 3) "The most robust system that works in all sorts of current realities in West Africa". For instance, frequent electricity shortcuts may occur, or internet connection may be poor or unstable. For the H-TEP session specifically, we defined one additional strategy: 4) "The system that requires the least resources for West African end users" (e.g., skilled personnel, good internet connection, good / constant power supply).

Additionally, we performed a combination of "Brainwriting 635" (Litcanu et al., 2015;Paulus and Yang, 2000) with the "Cadavre Exquis" game. This consists of a collection of words, written on a paper by a participant. It is then folded and handed over to the next person. The aim of this session was thus to also interactively develop additional system configurations for the IVP using the same strategies as in the Strategy Generation Table sessions. The Brainwriting 635 method allowed the participants to extensively discuss the system characteristics before deciding. In contrast to the Strategy Generation Table session, the participants decided alone on the characteristics of one single system element, independently of the other elements, only knowing the strategy. These results were used to consolidate the configurations developed with the Strategy Generation Table.

After all three parallel sessions, results were discussed in a plenary session. As post-processing, the strategies from all sessions were combined to characterize the entire FANFAR system, i.e., combining both the IVP and H-TEP part. Two experts from SMHI and Eawag later discussed and defined further system configurations to cover technically interesting system configurations.

\subsubsection{Strategy Generation Table}

We adapted the "Strategy Generation Table" method from Gregory et al. (2012). Each session was moderated by two FANFAR consortium members. The moderators prepared big sticky notes with possible system configuration "elements" and smaller sticky notes with characteristics for each "element". The moderator and participants built a table with the big sticky notes (elements) as headers, and possible characteristics for each element in rows (example see Table SI-1). The system configuration elements were discussed independently from each other (i.e., characteristics of column A were created independently of column B).

Table SI-1. Example of Strategy Generation Table: system configuration elements (columns A-D) and corresponding characteristics (rows a - e belonging to each column) to generate suitable system configurations for the Interactive Visualization Portal. Note: an 
unequal number of characteristics can be used. This example is a shorter representation of the table actually generated in workshop 1.

\begin{tabular}{|c|c|c|c|c|c|c|}
\hline & A & B & C & D & & $F$ \\
\hline & Forecasted variables & Observed variables & $\begin{array}{l}\text { Model performance } \\
\text { accuracy }\end{array}$ & I Data download & $\begin{array}{l}\text { Distribution } \\
\text { channels }\end{array}$ & Language \\
\hline$a$ & River discharge & None & $\begin{array}{l}\text { No performance metrics } \\
\text { shown }\end{array}$ & $\begin{array}{l}\text { cs No option to down- } \\
\text { load data }\end{array}$ & $\begin{array}{l}\text { Website with in- } \\
\text { teractive features }\end{array}$ & English \\
\hline b & $\begin{array}{l}\text { River discharge \& water } \\
\text { level }\end{array}$ & $\begin{array}{l}\text { Water level from satel- } \\
\text { lites }\end{array}$ & $\begin{array}{l}\text { Display performance } \\
\text { metric for forecasts }\end{array}$ & $\begin{array}{l}\text { Tabular data for se- } \\
\text { lected station in } \\
\text { TXT format }\end{array}$ & $\begin{array}{l}\text { Website with } \\
\text { static images }\end{array}$ & $\begin{array}{l}\text { English } \\
\text { French }\end{array}$ \\
\hline c & $\begin{array}{l}\text { River discharge, water } \\
\text { level, \& precipitation }\end{array}$ & $\begin{array}{l}\text { Water level from in- } \\
\text { situ measurements } \\
\text { and satellites }\end{array}$ & $\begin{array}{l}\text { Blank out areas where } \\
\text { forecasting performance } \\
\text { is too low }\end{array}$ & $\begin{array}{l}\text { Tabular data for se- } \\
\text { ce lected station in Ex- } \\
\text { cel format }\end{array}$ & $\begin{array}{l}\text { Website with text } \\
\text { summaries only }\end{array}$ & $\begin{array}{l}\text { English, French, } \\
\text { and Portuguese }\end{array}$ \\
\hline $\mathrm{d}$ & $\begin{array}{l}\text { River discharge, water } \\
\text { level, precipitation, \& } \\
\text { evaporation }\end{array}$ & $\begin{array}{l}\text { River discharge from } \\
\text { in-situ measurements }\end{array}$ & & $\begin{array}{l}\text { Map of displayed } \\
\text { variable(s), in PNG } \\
\text { format }\end{array}$ & $\begin{array}{l}\text { SMS alert notifi- } \\
\text { cations }\end{array}$ & $\begin{array}{l}\text { English, French, } \\
\text { Portuguese, Ara- } \\
\text { bic }\end{array}$ \\
\hline e & $\begin{array}{l}\text { River discharge, water } \\
\text { level, precipitation, evapo- } \\
\text { ration, soil moisture stor- } \\
\text { age }\end{array}$ & $\begin{array}{l}\text { In-situ water level and } \\
\text { - river discharge, and } \\
\text { - water level from satel- } \\
\text { lites }\end{array}$ & & $\begin{array}{l}\text { Map of displayed } \\
\text { variable(s), in } \\
\text { Shapefile format }\end{array}$ & $\begin{array}{l}\text { Email alert notifi- } \\
\text { cations }\end{array}$ & \\
\hline
\end{tabular}

In the H-TEP session, the following system configuration elements were discussed:

- Input data: "diversity of observational data sources", "data processing options (input data)", and "meteorological input / forcing data

- Related to the models: "number of models used"

- Outputs: "type of information derived / forecast outputs from HTEP"

- Distribution channels: "distribution options from TEP"

- Additional tool features: "degree of automatization", "language", and "support system".

In the IVP session, the main elements were: "Forecasted variables"; "Observed variables"; "Model performance / accuracy"; and "Reference thresholds to compare with". Additional tool features were also discussed, namely "Data download"; "Distribution channels"; "Language"; and "Alert notification system".

Thereafter, workshop participants formulated strategies to define different FANFAR system configurations. In this context, a strategy is a logically consistent set of characteristics (of every element) that are combined to create a comprehensive system option (i.e., FANFAR system configuration) through a recognizable question or driver (Howard, 1988). Thus, for each strategy one characteristic from each column was selected. In both sessions, three strategies were used as guiding question (example see Figure SI-1).

\footnotetext{
Strategy 1: Most easy-to-use $\rightarrow$ What is the most easy-to-use system? (What could your neighbor use?).

Strategy 2: Most attractive $\rightarrow$ What is the most attractive system for West-Africa?

Strategy 3: Most robust

$\rightarrow$ What is the most robust system that works in all sorts of current realities in West Africa?

In the H-TEP session, an additional strategy was created. This "Least resources" strategy refers to a system that requires the least resources for West-Africa (note: not the least resources for the FANFAR consortium or SMHI), e.g., skilled personnel, good internet connection, and good / constant power supply.
} 


\begin{tabular}{|c|c|c|c|c|c|c|}
\hline & $\mathbf{A}$ & B & C & D & $E$ & $F$ \\
\hline & $\begin{array}{c}\text { Forecasted } \\
\text { variables }\end{array}$ & $\begin{array}{l}\text { Observed } \\
\text { variables }\end{array}$ & $\begin{array}{c}\text { Model } \\
\text { performance / } \\
\text { accuracy }\end{array}$ & $\begin{array}{c}\text { Data } \\
\text { download }\end{array}$ & $\begin{array}{c}\text { Distribution } \\
\text { channels }\end{array}$ & Language \\
\hline$a$ & River discharge & None & $\begin{array}{l}\text { No performance } \\
\text { metrics shown }\end{array}$ & $\begin{array}{l}\text { No option to } \\
\text { download }\end{array}$ & $\begin{array}{l}\text { Website } \\
\text { with }\end{array}$ & \\
\hline $\begin{array}{l}\text { The one that } \mathrm{c} \\
\text { implemented } \mathrm{f}\end{array}$ & $\begin{array}{l}n \text { be } \\
\text { ster. }\end{array}$ & & & & $\begin{array}{l}\text { interactive } \\
\text { features }\end{array}$ & / \\
\hline $\mathrm{b}$ & $\begin{array}{l}\text { River discharge \& } \\
\text { water level }\end{array}$ & $\begin{array}{c}\text { Water level } \\
\text { from satellites }\end{array}$ & $\begin{array}{l}\text { Display } \\
\text { performance } \\
\text { metric for } \\
\text { forecasts }\end{array}$ & $\begin{array}{c}\text { Tabular data } \\
\text { for selected } \\
\text { station in } T X T \\
\text { format }\end{array}$ & $\begin{array}{c}\text { Website } \\
\text { with static } \\
\text { images }\end{array}$ & $\begin{array}{l}\text { E/glish and } \\
\text { French }\end{array}$ \\
\hline c & $\begin{array}{c}\text { River discharge, } \\
\text { water level, \& } \\
\text { precipitation }\end{array}$ & $\begin{array}{l}\text { Water level } \\
\text { from in-situ } \\
\text { measurements } \\
\text { and satellites }\end{array}$ & $\begin{array}{c}\text { Blank out areas } \\
\text { where forecasting } \\
\text { performance is } \\
\text { oo lov }\end{array}$ & $\begin{array}{l}\text { Tabular data } \\
\text { for selected } \\
\text { station in } \\
\text { Excel format }\end{array}$ & $\begin{array}{c}\text { SMS alert } \\
\text { notifications }\end{array}$ & $\begin{array}{l}\text { English, } \\
\text { French, and } \\
\text { Portuguese }\end{array}$ \\
\hline $\mathrm{d}$ & $\begin{array}{c}\text { River discharge, } \\
\text { water level, } \\
\text { precipitation, \& } \\
\text { evaporation }\end{array}$ & $\begin{array}{l}\text { River discharge } \\
\text { from in-situ } \\
\text { measurements }\end{array}$ & & $\begin{array}{c}\text { Map of } \\
\text { displayed } \\
\text { variable(s), in } \\
\text { PNG format }\end{array}$ & $\begin{array}{l}\text { Enfail flert } \\
\text { notifications }\end{array}$ & $\begin{array}{l}\text { English, } \\
\text { French, } \\
\text { Portáguese, } \\
\text { Arabic }\end{array}$ \\
\hline $\begin{array}{l}\text { e } \\
\text { The most d } \\
\text { an ideal wo }\end{array}$ & $\begin{array}{l}\text { River discharge, } \\
\text { sired in level, } \\
\text { d? itation, } \\
\text { d? moisture storage }\end{array}$ & $\begin{array}{c}\text { In-situ water } \\
\text { level and/iver } \\
\text { discharge, and } \\
\text { water level from } \\
\text { satellites }\end{array}$ & & $\begin{array}{l}\text { abular data } \\
\text { in XXT format } \\
\text { and map } \\
\text { displayeuiir } \\
\text { PNG format }\end{array}$ & $\begin{array}{l}\text { Website } \\
\text { with } \\
\text { interaglive } \\
\text { features, } \\
\text { SMS and } \\
\text { Email }\end{array}$ & \\
\hline
\end{tabular}

Figure SI-1. Example of creating strategies to generate system configurations for the FANFAR Interactive Visualization Portal. Examples of system configuration: "The strategy that can be implemented fastest" (red); "The most desired strategy in an ideal world" (green).

\subsubsection{Brainwriting 635 combined with Cadavre Exquis}

The session combining "Brainwriting 635" (Litcanu et al., 2015;Paulus and Yang, 2000) with the "Cadavre Exquis" game was also moderated by two people. There were 17 French speaking workshop participants. This group aimed at creating plausible FANFAR system configurations of the Interactive Visualization Portal (IVP). The approach followed the same structure as the "Strategy Generation Table" groups. Brainwriting 635 was used as interactive brainstorming method to develop characteristics for each element of possible FANFAR system configurations (see sect. SI-1.1.1 for details concerning elements and characteristics). Session participants were split into three smaller groups. Each group discussed six elements: "Observed variables", "Model performance / accuracy", "Forecasted variables", "Reference thresholds to compare with", "Representation of variables", and "Distribution channels". After this part, a speaker was elected in each group to highlight the results to the other groups.

Thereafter, the Cadavre Exquis game was used to generate system configurations for the IVP. The same three guiding questions were used as for the Strategy Generation Table sessions (sect. SI-1.1.1). Each group tackled only one question. The characteristics developed and discussed during the Brainwriting 635 part (with the exception of "Model performance / accuracy") were now selected in such a way that they were suitable for the guiding question, e.g., "What is the most attractive system for WestAfrica?" For this, each participant selected the most suitable characteristic for one element, wrote it on a sheet of paper, and handed it to her neighbor. This person now selected the most suitable characteristic for the next element, and handed the folded paper to his neighbor. The Cadavre Exquis game is thus a structured way of asking each participant to choose a characteristic for the next system configuration element without knowing the previous selections (they only know the strategy). 


\subsection{Eliciting weights}

We elicited weights in five groups in the second FANFAR workshop (Table SI-2). More information see main text.

Table SI-2. Overview of stakeholder groups and weight elicitation method used during the second FANFAR workshop. Group-ID abbreviation: G1 - G5 = group number, A / B = sub-group, EM = emergency managers, HY = hydrologists, $F / E=$ language.

\begin{tabular}{lllll}
\hline \hline Group-ID & Stakeholder type & Language & No. of participants & Method \\
\hline G1A_EM_F & EM & F & 8 & Swing \\
G2A_HY_F & HY & F & Sub-group of total 11 & Swing \\
G2B_HY_F & HY & F & Sub-group of total 11 & Swing \\
G3A_HY_E & HY & E & 14 & Simos card \\
G4A_EM_E & EM & E & 3 & Simos card \\
G5A_AGRHYMET & AGRHYMET & F and E & 3 & Simos card \\
\hline \hline
\end{tabular}

\subsubsection{Swing weight elicitation}

We used a bottom-up hierarchical standard Swing weight elicitation method (Eisenführ et al., 2010) for the French speaking workshop participants. We describe the procedure here in some detail for readers not familiar with Decision Analysis methods. We first carried out the weight elicitation on the lower-level objectives of the objectives hierarchy, one branch after the other until all four branches were covered (i.e., two sub-objectives belonging to "High information accuracy and clarity", three from "Good information access", two from "Low costs", and three from "High sustainability"). In Swing, the stakeholders first order all objectives in question (i.e., those from one branch) in terms of importance, answering the question: "Imagine that al objectives are on their worst possible level. You can now choose to "swing" one to its best level. Which objective is most important to you to improve to its best possible level?" After setting all objectives back to the worst level, the second most important objective is moved to the best possible level, and so on. Note: it is very important that the stakeholders understand the objectives and attributes, which need to be explained, along with the ranges (worst and best possible case). After ranking objectives, they are rated. Per default, the hypothetical option, where the most important objective is on its best level, and all others on their worst levels, receives 100 points. The hypothetical option with all objectives being on their worst level receives 0 points. The stakeholders are asked to assign points to the hypothetical options in between, which reflect their preferences For instance, if they assign 50 points to the hypothetical option where the second most important objective is on its best level, it is half as important to them to improve this objective to its best level, compared to the most important objective.

After having done this for each hierarchy branch, we repeated the procedure for the higher-level objectives across the hierarchy. We used the most important lower level objective of each branch identified in the first step. To check for consistency, we repeated the procedure across the hierarchy for the higher level objectives using the second most important lower level objectives.

Stakeholders can be uncertain about their preferences or in group sessions, there may be disagreement among group members about the exact numbers. Therefore, we allowed the participants to state a range of swing points representing their uncertainty. We took the mean of these ranges as main preference statement and considered the ranges in the later sensitivity analyses.

We transformed the swing points to local weights $\boldsymbol{w}_{\boldsymbol{r}}$ (i.e., the weight of the lower level objective relative to the other lower level objectives within the same branch; eq. SI-1; note that the sum of all local weights in one branch equals 1), using following formula (Eisenführ et al., 2010):

$$
w_{r}=\frac{t_{r}}{\sum_{i=1}^{m} t_{i}}
$$

$\mathrm{w}_{\mathrm{r}}=$ local weight of objective $\mathrm{r} ; \mathrm{t}_{\mathrm{r}}=$ swing points of objective $\mathrm{r} ; \mathrm{t}_{\mathrm{i}}=$ swing points of objectives within same branch; $\mathrm{m}=$ number of objectives within branch 
Second, we calculated the global weight $W_{r}$ (i.e., the weight of the lower level objective relative to all other lower level objectives; eq. SI-2) by multiplying the local weight of the lower-level objective by the local weight of the respective higher-level objective (Marttunen et al., 2018). Note that the sum of all global weights equals one.

$$
W_{r}=w_{L 1_{r}} \times w_{L 2_{r}}
$$

$\mathrm{W}_{\mathrm{r}}=$ global weight of objective $\mathrm{r} ; \mathrm{w}_{\mathrm{L} 1 \mathrm{r}}=$ local weight of lower-level objective $\mathrm{r} ; \mathrm{t}_{\mathrm{L} 2 \mathrm{r}}=$ local weight of higher-level objective which includes sub-objective $\mathrm{r}$

We translated the ranges of uncertainty stated during the assignment of the swing points into weight ranges using the same formula. These were used to inform the later sensitivity analyses. Below, we describe our procedure in some more detail:

The minimum local weight resulted from considering the minimum Swing points for the given objective, and the maximum Swing points for all other objectives within the same branch. Likewise, the maximum local weight resulted from the maximum Swing points of the objective, and the minimum Swing points of the other objectives. A justification for this approach is that sensitivity analyses should exactly challenge the assumptions of the model, in this case the weight preference statements, by covering (plausible) extremes. We calculated the ranges of the global weight for each objective by multiplying the limits of the local weight of the lower-level objective by the limits of the local weight of the corresponding higher level objective.

The minimum global weight $\left(W_{r_{\text {min }}}\right)$ was calculated by eq. SI-3:

$$
W_{r_{\text {min }}}=w_{L 1_{r_{\text {min }}}} \times w_{L r_{\text {min }}}
$$

Where the minimum local weight of the lower level objective $w_{L 1_{r_{m i n}}}$ resulted from considering the lowest value of Swing points for that objective and the highest value of Swing points for the other objectives within that branch. Likewise, the minimum local weight of the higher level objective $w_{L 2_{r_{m i n}}}$ resulted from considering the minimum value of Swing points assigned to this higher level objective and the maximum value of Swing points assigned to all other higher-level objectives.

Likewise, we calculated the upper limit of the range in weights for each objective by considering the scenario that results in the highest possible global weight for that objective. The maximum global weight $\left(W_{r_{\max }}\right)$ was calculated by eq. SI-4:

$$
W_{r_{\max }}=w_{L 1_{r_{\max }}} \times w_{L r_{r_{\max }}}
$$

As stated above, this method results in the largest possible range of global weights given the preference statements of the participants. However, the most extreme global weights of the range $\left(W_{r_{\min }}\right.$ and $\left.W_{r_{\max }}\right)$ are multiplications of the extremes of uncertainty of different individual preference statements and therefore are unlikely. The extremes should be interpreted as boundary of possible uncertainty and serve as qualitative indication of which objectives for each stakeholder group require a sensitivity analysis on weights.

\subsubsection{Simos' revised card procedure}

In three sessions, we used an adaptation of the revised Simos' procedure to elicit weights with cards (Figueira and Roy, 2002; Pictet and Bollinger, 2008). Again, we describe the procedure here for readers not so familiar with Decision Analysis methods. First, we presented the different objectives including worst/best cases (range) to the participants on sticky notes, and asked them to stick the notes onto a blank wall ordering them side-by-side according to their importance. Note that we did not introduce numbers or give any signals that could lead to an obvious bias, e.g., numbering the objectives in whatever predefined order. Whenever there were two or more objectives with the exact same importance, they were put in the same position.

After rank-ordering all objectives on the wall, the moderator assigned a default score of 100 to the most important objective and asked the group members for a score between $0-100$ for the least important objective. This allowed to obtain the range (in points) between least important (chosen points) and most important objective (per default 100 points). Later, the ratio of points of the most important objective (100) to the points of the least important objective will be called $Z$ (see below). The $Z$ value thus indicates how many times the most important objective is more important than the least important objective. In this step, the moderator allowed for a range for the score on the least important objective. 
Second, we tasked the group members to discuss the difference in importance of successively ranked objectives. They were asked to stick blank notes between objectives to represent the relative differences in importance between successive objectives (i.e., the more sticky notes between two objectives, the larger the difference in importance). The moderator asked questions to check consistency regarding the blank sticky notes, e.g., between two non-consecutive objectives, and the score given to the least important objective, e.g., if the most important objective was x times more important that the least important objective.

To translate the resulting composition of sticky notes on the wall into weights, we first calculated the $Z$ value. This is the ratio of the score of the most important objective (100 per definition) by the score of the least important objective assigned by the group. Thereafter, we first calculated the initial weight $O_{r}$ of each objective (eq. SI-5), and afterwards rescaled the weights to achieve the final global weights $W_{r}$ (eq. SI-6), which sum up to one (Pictet and Bollinger, 2008):

$$
O_{r}=r_{\min }+(Z-1) \frac{r-r_{\min }}{r_{\max }-r_{\min }}
$$

$\mathrm{O}_{\mathrm{r}}=$ initial weight of objective $\mathrm{r} ; \mathrm{Z}=$ ratio of most important objective and least important objective; $r=$ inverse rank; $r_{\text {min }}=$ def. $1 ; \mathrm{r}_{\max }=$ maximum rank

$$
W_{r}=\frac{1}{\sum_{i=1}^{n} o_{i}} \times O_{r}
$$

$\mathrm{W}_{\mathrm{r}}=$ global weight of objective $\mathrm{r}$; $\mathrm{O}_{\mathrm{r}}=$ initial weight of objective $\mathrm{r}, \mathrm{n}=$ number of objectives

As the groups were allowed to state a range for the score of the least important objective, we translated the min/max boundaries of that range into $Z$ values as well. The moderator decided on a value within the range to focus the analysis on. In addition to the weight set resulting from the focus $Z$ value, we calculated the individual weights using the $\min / \max$ values of the $Z$-value. This resulted in a total of three different weight sets, in each set the weights sum up to one. The set resulting from the focus $Z$ value was used for the main MCDA, while the other two sets were incorporated in sensitivity analyses (sect. SI-1.4).

\subsubsection{Observations from weight elicitation informing sensitivity analyses}

To examine for which groups and objectives sensitivity analyses should be carried out, we gathered all comments documented by the moderators (Table SI-3). This allows us to understand, where uncertainties in the elicited preferences are to be expected.

Table SI-3. Summary of remarks on preferences during weight elicitation and uncertainty for each stakeholder group (SH-Group).

\section{SH-GROUP SUMMARIZED REMARKS ON PREFERENCE STATEMENTS AND UNCERTAINTY}

G1_EM_F Weight elicitation on lower level objectives of hierarchy: Consensus on Swing points, uncertainty ranges given on (SWING) Some objectives $\rightarrow$ sensitivity analysis on uncertainty ranges.

Weight elicitation on higher level objectives (comparison of most important objective of each branch): There was a long discussion. Result: give equal weight to each top-level objective, because of connected chain in emergency response that needs all four main objectives. They are all equally important. $\rightarrow$ No uncertainty in this statement; no sensitivity analysis needed.

Consistency check on higher level objectives (comparison of second most important objective of each branch): This consistency check did not seem to work properly, which was also an elicitation problem. It would have been necessary to discuss inconsistencies to initial weight elicitation, but there was no time. Proposal of moderator (JL): regard first set of weights from initial elicitation as "good set" and do sensitivity analysis with second set from consistency check. $\rightarrow$ sensitivity analysis with different weight set from consistency check.

Group comments and reasoning concerning preferences on objectives within branches:

High information accuracy and clarity: From the point of view of the emergency managers it is more important to have clear information than highly precise information (this may be different for hydrologists).

Good information access: We have to receive the information BEFORE the flood. This is more important than the reliable distribution channels.

Low costs: It must possible that we can finance / pay / buy the product; we can wait for a good product.

High sustainability: If there are no people in West Africa that can use and maintain the system, the financing is of no use; the system does not help us in such a case. 


\section{SH-GROUP SUMMARIZED REMARKS ON PREFERENCE STATEMENTS AND UNCERTAINTY \\ G2_HY_F Weight elicitation on lower level objectives: Two groups of different opinion for the two sets of objectives within (SWING) branches "High information accuracy and clarity" and "High sustainability". Consensus on the other two sets of objec- tives within branches "Good information access" and "Low costs". Both groups gave a range of Swing points for most lower-level objectives. $\rightarrow$ Sensitivity analysis on uncertainty ranges. \\ Weight elicitation on higher level objectives (conducted for each preference set for the lower level objectives): Two groups of different preferences for higher level objectives with different ranking of higher level objectives and dif- ferent ratings (Swing points) including uncertainty ranges. Not sure if the participants were always the same ones in these groups with different preference sets. Both groups gave a range of Swing points for some higher level objectives. $\rightarrow$ Sensitivity analysis on uncertainty ranges.}

Group comments and reasoning concerning preferences on objectives within branches:

High information accuracy and clarity: Group 1: the (un-) precise information is really the most important problem we have; Group 2 was a minority who found it more important to have clear risk information

Good information access: The ranking was very clear. For objective 2, there was disagreement about the importance (points). Some said: objective 2 is nearly as important as 1 because you really need the information on time; Weight of objective 3: everybody easily agreed.

Low costs: They all agreed. They explained: if we can improve objective 1 (short development time), we can save lives; which is why this objective is more important.

High sustainability: There was a relatively long discussion concerning the order (ranking) of the objectives, which is why the group was split up into two groups. Reasoning of some: we can have all the money we like (objective 1), but if we do not have qualified people, the money is of no use. Others said: with money, we can also employ qualified people; no money, then nothing works. There was no discussion about the third objective, but they wanted agreed on a range.

G3_HY_E Some disagreement on certain values: solved by registration of all contributions and specific value assigned by majority (SIMOS CARD) rule. One specific participant from NIHSA had a slightly different preference set. The remaining participants had very similar preferences.

Z-value range from 5 to 20 , focus on 10 (decided by moderator) $\rightarrow$ sensitivity analysis on Z-value range.

G4_EM_E Very small group. Easy to discuss in detail and explain objectives and their ranges well, while focusing on objectives (SIMOS CARD) that were difficult to understand.

Z-value range from 3.33 to 5 , focus on 3.33 (decided by moderator) $\rightarrow$ sensitivity analysis on Z-value range.

G5_AGRHY-MET Bilingual session EN and FR. Small and very fast group. Some participants helped translate objectives and understood (SIMOS CARD) them fast. Participants work together and know each other well.

Z-value range from 3.33 to 5 , focus on 5 (decided by moderator) $\rightarrow$ sensitivity analysis on Z-value range.

The Swing weight elicitation resulted in many more remarks from the moderator on uncertainty and differing opinions within groups, than from the Simos card method. This is not a property of the respective method but due to the fact that two different moderators elicited the weights. In the Swing process, the moderator actively encouraged the participants to discuss and state their differing opinions, which then were separately documented. In Simos card method, the moderator requested the group to reach a common opinion, and only this consensus was documented.

Because of very strongly differing weight sets of one Swing group, we decided to split the group consisting of French hydrologists into two separate stakeholder groups for the analysis. One preference set was assigned to the group G2A_HY_F and the opposing opinion formed the group G2B_HY_F.

During the weight elicitation of the French speaking emergency managers (G1A_EM_F) there was a consensus of all group members on the ranking and weighting of the objectives on both levels of the hierarchy. However, the consistency check conducted on the higher-level objectives (using the second-most important objective) resulted in a preference statement that was inconsistent with the initial weight elicitation. The time restrictions during the workshop did not allow to discuss the inconsistence with the participants and resolve the issue. Therefore, we split this group into two sub-groups for the analysis: Sub-group 1 with the preference set that resulted from the initial weight elicitation, and sub-group 2 with the preference set for the higher-level objectives, which resulted from the consistency check. For the MCDA analysis, we used the weight set of 
Sub-group 1 and conducted a sensitivity analysis with the weight set of Sub-group 2 to check whether the MCDA results differ for the two sub-groups.

In the English-speaking groups (G3A_HY_E, G4A_EM_E, and G5A_AGRHYMET) that used the Simos card method, the moderator requested the group members to reach a consensus within each group. Therefore, there were no differing preference sets elicited within these groups and consequently we did not form sub-groups.

\subsection{MCDA aggregation model}

We used an unconventional non-additive aggregation model to integrate the predictions and stakeholder preferences in the MCDA (see main text). The properties and advantages of the weighted power mean model are extensively discussed in (Haag et al., 2019). The implications are visualized for two dimensions in Figure SI-2, i.e., for two exemplary objectives only. Using the additive aggregation model (weighted arithmetic mean) would result in linear isolines (right plot), while a nonlinear model results in curved isolines (left plot). As a consequence, for the power mean aggregation model, if one attribute is on a very bad level (i.e., value is low), the total value after aggregation is also low (red coloring), even if the second attribute achieves a very good level (i.e., high value). At the other extreme, if both attributes each achieve a good level (i.e., high value), the aggregation model is close to linear (blue coloring). In additive aggregation, a poor achievement on one attribute can be fully compensated if the other attribute achieves a good level. The ValueDecisions app (Haag et al., subm.) has the advantage that different aggregation models can easily be implemented, which allows testing the sensitivity of the results to model assumptions.
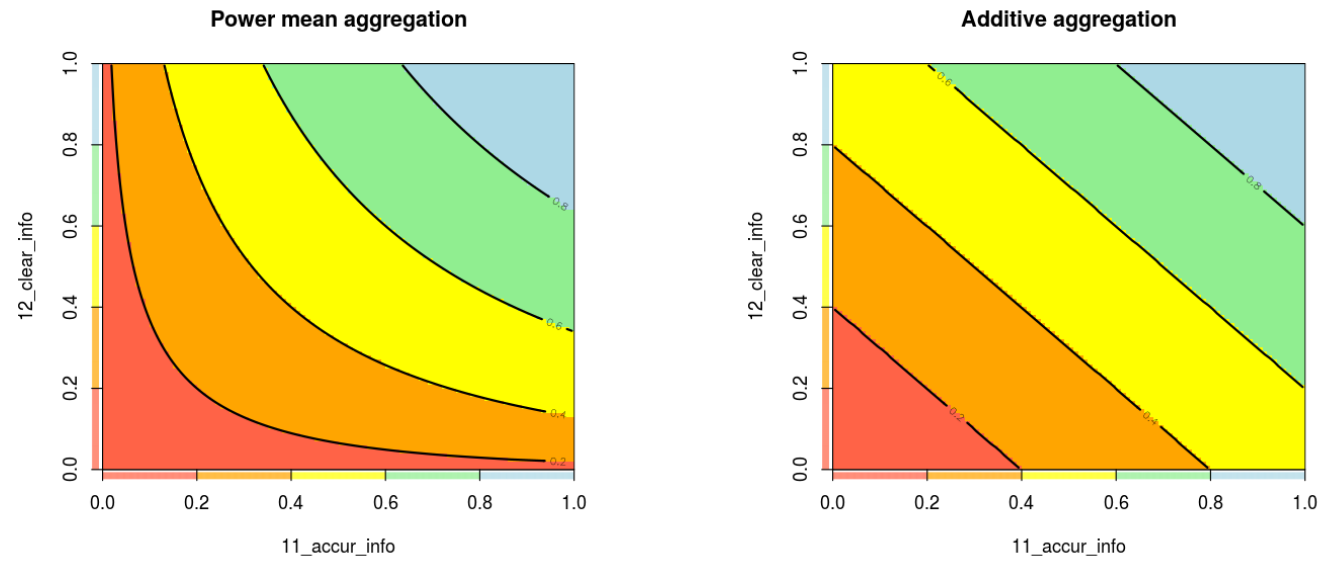

Figure SI-2. Visualization of the power mean aggregation model with $\gamma=0.2$ as utilized in our MCDA (left) and the commonly used additive aggregation model, or weighted arithmetic mean (right; which can be inferred from the power mean model if $\gamma=1$ ). Displayed here is a hypothetical case where the two objectives 11_accur_info, and 12_clear_info are aggregated with equal weights. The axes represent the option's (i.e., FANFAR system configurations') achieved value on the two objectives. The isolines (or any other x-ypoint in the plot) indicate the option's total value after aggregation of the two objectives.

\subsection{Sensitivity analyses on elicited weights and aggregation model}

It is common to use local sensitivity analyses to check the sensitivity of the MCDA results to diverging preferences (e.g., Eisenführ et al., 2010;Zheng et al., 2016), or to model assumptions. We checked the sensitivity of our results to other aggregation models, and to other weights, since there was not always consensus concerning the exact numbers among group members of the weight elicitation workshops (see sect. SI-1.2.3, and Table SI-3). We give the settings of sensitivity analyses in the Results (Error! Reference source not found. main text), with setting S0 as default.

To test implications of the aggregation model (see main text, sect. 2.2.9), we recalculated the MCDA for other reasonable models (Haag et al., 2019), running a new MCDA for each. Applying alternative models requires one click in the ValueDecisions app (Haag et al., subm.).

During weight elicitation, consistency checks are carried out. For the group of French speaking emergency managers (G1A_EM_F), the consistency check revealed an inconsistency for the weighting of the higher-level objectives, which was 
not possible to resolve during the workshop (Table SI-3). The difference between the weight sets of these two sub-groups were large, especially for the higher level objectives 2_access, and 3_costs (Figure SI-3). Therefore, we examined the effect of the alternative weight set (sub-group 2) on the MCDA results in a sensitivity analysis (sensitivity analysis S21).
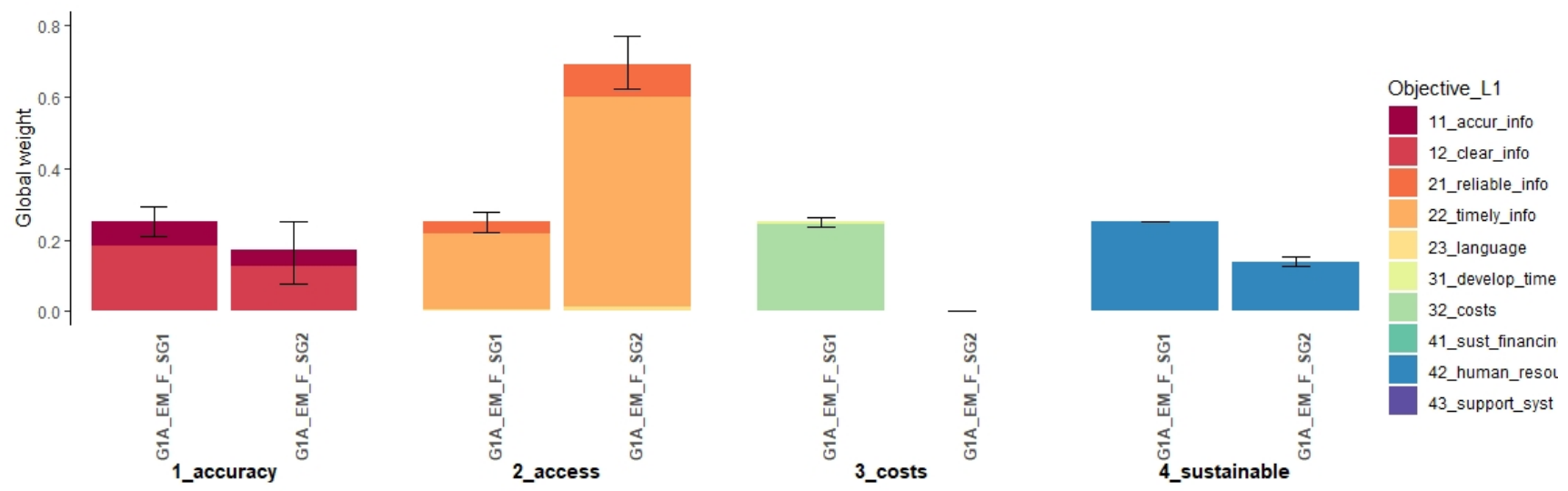

Figure SI-3. Comparison of global weights (y-axis) from the two sub-groups within the French speaking emergency managers, where the consistency check revealed inconsistent weight preferences. For each higher level objective (e.g., 1_accuracy, 2_access, ...), sub-group 1 is depicted to the left (G1A_EM_F_SG1), and sub-group 2 to the right (G1A_EM_F_SG2). Colored stacked bars indicate lower level objectives. Error bars denote the minimum and maxim weights, which result from the ranges in points given by stakeholders in Swing weight elicitation.

Our approach for the further sensitivity analyses depended on the method used to elicit the weights. For Swing weight elicitation, we had allowed the workshop participants to state ranges rather than giving precise numbers if they wished. In this case, a sensitivity analysis towards weight changes of each individual objective was required (sensitivity analyses S22). We defined a threshold of $\Delta=0.02$ for the ranges; i.e., the difference of the maximum or minimum weight from the average global weight had to be greater than 0.02 , else we did not expect a strong effect on the MCDA results. The cases exceeding the defined threshold are highlighted in Table SI-31. For these cases, we visually investigated whether the respective objective (for the respective stakeholder group) was prone to be sensitive towards weight changes. This is easily possible in the ValueDecisions app, which provides weight sensitivity plots with a mouse click. These plots visualize for which configurations severe changes in values are to be expected if the weight of the objective is altered (for the respective stakeholder group).

An example of these sensitivity plots is provided for the objectives of stakeholder group G2B_HY_F (Figure SI-4). We identified several cases of weight ranges exceeding the threshold of $\Delta=0.02$. Consulting the weight sensitivity plots, we could exclude three cases (21_reliable_info, 22_timely_info, 41_sust_financin), which were not sensitive towards weight changes in the corresponding objectives (highlighted green in Table SI-31). However, we identified two objectives (11_accur_info and 12_clear_info) which seemed to be sensitive towards weight changes.

Thereafter, we individually tested each identified case by recalculating the MCDA. As input into the MCDA model, we utilized the maximum, respectively minimum weight of the sensitive objective, as given by the ranges in the sessions with stakeholders. In the MCDA, the ratios of the weights of all the other objectives are kept constant, and are then normalized to keep the sum of all weights equal to 1 . This is done automatically in the ValueDecisions app if new weights are entered for local sensitivity analyses. For a thorough explanation of the method we refer to Eisenführ et al. (2010). Thus, we reran the ValueDecisions app with the new weights and re-calculated the aggregated values of all system configurations (local sensitivity analyses (S22_11_min/max and S22_12_min/max). The results then reveal whether larger changes in the values of configurations and/or rank reversals of the configurations are provoked by these weight changes.

For the three stakeholder groups whose weights were elicited using Simos' card method, the sensitivity analyses on weight ranges were more straightforward. The Z-value was elicited as range (sect. SI-1.2.2). We recalculated MCDA results for each group, using the two additional weight sets resulting from the minimum, respectively maxim Z-value (sensitivity analysis S231 and S232). For each group, we compared these two additional MCDA results with the one resulting from the weight set of the focus Z-value and identified any major changes in the system configurations' values and any apparent rank reversals. Using the maximum $\mathrm{Z}$ value was only necessary for group G3A (S232), because $\mathrm{Z}$ min was used as focus value for groups G4A and G5A (i.e., as S0). 
It is common to test other interesting objectives by doubling the elicited weight. We did this for "Several languages", because its importance might have been underestimated (S31). For each setting, we compared results (i.e., ranks of configurations) with those of the default MCDA (S0; Table 3, main text), using the nonparametric Kendall's $\tau$ correlation coefficient (Kendall, 1938) to measure rank reversals (as in Zheng et al., 2016). As input in the correlation analyses, we used the mean ranks resulting from 1'000 Monte Carlo simulation runs with the changed parameter settings.

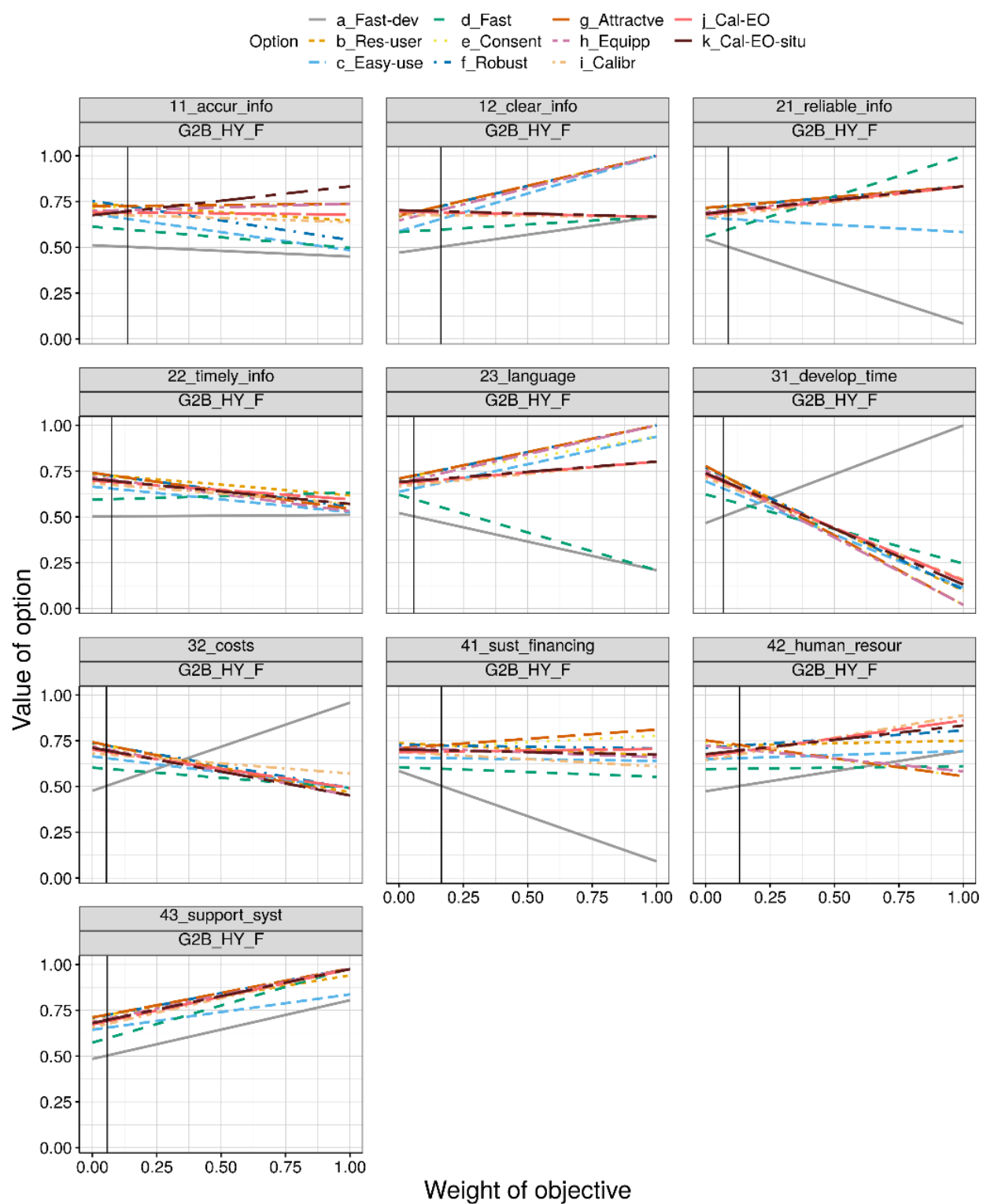

Figure SI-4. Weight sensitivity plots for stakeholder group G2B_HY_F. For each objective (boxes), the weight (x-axis) can vary between 0 (objective does not count in this decision) to 1 (only this objective is important, all other objectives do not count). Colored lines: FANFAR configurations, and the value ( $y$-axis) that is achieved by an configuration for a given weight. Vertical black line: actual weight assigned by this stakeholder group. If configuration value lines intersect near the vertical line, a sensitivity of the configurations' ranking towards weight change is to be expected. If configuration value lines run parallel or diverge, no sensitivity towards weight changes is to be expected.

\subsection{Discuss results with stakeholders, feedback}

We organized two half day online rainy season reflection workshops from 21-22 January 2021, inviting the same stakeholders from the 17 West and Central African countries that were invited during the three co-design workshops. The workshops took place on the Zoom platform (https://zoom.us/), with simultaneous translation between English and French. The aim of the reflection workshops was to share experiences with floods and the use of the FANFAR system over the 2020 rainy season. 
During the first workshop day, stakeholders were given one hour to fill out an online survey (provided in English and French) on the Limesurvey platform (https://www.limesurvey.org/). Using this survey, we aimed to elicit perceived and expected performance of the FANFAR system. To this end, the survey contained three questions for each of the 10 objectives:

a. How much does FANFAR currently fulfil this objective?

b. Would you use the FANFAR system in the future if it remains as is?

c. What is the minimum acceptable to you? This means: below which level would you NOT use the FANFAR system?

Answers to questions a and c could be provided using a 7-point Likert scale for objective 11_accur_info, and a 5-point Likert scale for all other objectives. The results generated by Limesurvey were shared and discussed during the second workshop day. In addition to the direct question $b$, data were analyzed to assess whether the FANFAR system is perceived to meet each objective by assigning descending numbers between 1-5 (or 1-7) to Likert scale answers from best to worst. Then, the answer of $\mathrm{c}$ was subtracted from the answer of a for each response $(\mathrm{c}-\mathrm{a})$. 


\section{Supplementary Information: Results}

\subsection{Stakeholder analysis}

Table SI-4. Overview of the 68 stakeholders that were mentioned to play a role in developing the FANFAR forecast and alert system. The columns relate to the specific questions asked in the survey (see main text and details in Silva Pinto and Lienert (2018)); the task number is given in square brackets, e.g., task number 6 for importance of considering key West African organizations involved in producing and operating flood forecasts and early warning systems in co-design, and 14 for downstream stakeholders, respectively: [6, 14]. SH = stakeholder; Impt. = Importance of considering the SH's needs and interests; Infl. = Influence (power) in the implementation, Affect. = How strongly SH is affected by FANFAR system. The Likert scale for Impt., Infl., and Affect. ranges from 0 ("stakeholder has no influence/is not at all affected") to 10 ("stakeholder decides/ is very strongly affected by"); we present the average. Count $=$ total number of respondents that mentioned this SH in the survey. The survey was completed by 18 respondent groups, usually two (to three) people from the same country, with a total of 31 workshop participants.

\begin{tabular}{|c|c|c|c|c|c|c|c|c|c|}
\hline $\begin{array}{l}\text { Stakeholder } \\
{[1,9]}\end{array}$ & $\begin{array}{l}\text { Main interest } \\
{[3,11]}\end{array}$ & $\begin{array}{l}\text { Why use system } \\
{[4,12]}\end{array}$ & Information profile & Decisional level & Field & $\begin{array}{l}\text { Impt. } \\
{[6,14]}\end{array}$ & $\begin{array}{l}\text { Infl. } \\
{[7,15]}\end{array}$ & $\begin{array}{l}\text { Affect. } \\
{[8,16]}\end{array}$ & Count \\
\hline ABN & Resource planning & Forecast refinement & Hydro-innovation & Supranational & Water resources & 8,8 & 7,6 & 6,2 & 5 \\
\hline ABV & Resource planning & Forecast refinement & Hydro-innovation & Supranational & Water resources & 9,3 & 7,7 & 9,3 & 6 \\
\hline ACF & Rescue and aid & Alert information & Downstream & Supranational & Humanitarian aid & 6,0 & 6,0 & 5,0 & 2 \\
\hline ACMAD & Technical & Meteorological data & Hydro-innovation & Supranational & Meteorology & 8,3 & 8,3 & 8,0 & 6 \\
\hline AGRHYMET & Technical & Forecast production & Hydro-innovation & Supranational & Water resources & 9,8 & 9,1 & 8,7 & 10 \\
\hline ALG & $\begin{array}{l}\text { Economic service operations } \\
\text { and planning }\end{array}$ & Alert information & Downstream & Supranational & Agriculture & 4,0 & 4,0 & 2,0 & 1 \\
\hline ARC & $\begin{array}{l}\text { Economic service operations } \\
\text { and planning }\end{array}$ & Alert information & Downstream & Supranational & Other & 10,0 & 4,0 & 2,0 & 1 \\
\hline ASECNA & $\begin{array}{l}\text { Economic service operations } \\
\text { and planning }\end{array}$ & Meteorological data & Downstream & Supranational & Transportation & 6,0 & 4,0 & 5,0 & 1 \\
\hline CBLT & Resource planning & Forecast refinement & Hydro-innovation & Supranational & Water resources & 8,0 & 8,0 & 8,0 & 1 \\
\hline Community & Civil society & Alert information & Downstream & Local & Development & 6,6 & 4,3 & 10,0 & 7 \\
\hline CRS & Rescue and aid & Alert information & Downstream & Supranational & Humanitarian aid & 8,0 & 8,0 & 8,0 & 1 \\
\hline $\begin{array}{l}\text { Dam Management En- } \\
\text { tity }\end{array}$ & $\begin{array}{l}\text { Economic service operations } \\
\text { and planning }\end{array}$ & Water related information & Downstream & National & Energy & 8,2 & 7,6 & 8,4 & 5 \\
\hline ECOWAS & $\begin{array}{l}\text { Economic service operations } \\
\text { and planning }\end{array}$ & Other & Downstream & Supranational & $\begin{array}{l}\text { Industry and Com- } \\
\text { merce }\end{array}$ & 10,0 & 10,0 & 10,0 & 1 \\
\hline
\end{tabular}




\begin{tabular}{|c|c|c|c|c|c|c|c|c|c|}
\hline $\begin{array}{l}\text { Stakeholder } \\
{[1,9]}\end{array}$ & $\begin{array}{l}\text { Main interest } \\
{[3,11]}\end{array}$ & $\begin{array}{l}\text { Why use system } \\
{[4,12]}\end{array}$ & Information profile & Decisional level & Field & $\begin{array}{l}\text { Impt. } \\
{[6,14]}\end{array}$ & $\begin{array}{l}\text { Infl. } \\
{[7,15]}\end{array}$ & $\begin{array}{l}\text { Affect. } \\
{[8,16]}\end{array}$ & Count \\
\hline Educational Institution & Resource planning & Alert information & Hydro-innovation & National & $\begin{array}{l}\text { Research and edu- } \\
\text { cation }\end{array}$ & 0,0 & 0,0 & 10,0 & 1 \\
\hline Electricity Utility & $\begin{array}{l}\text { Economic service operations } \\
\text { and planning }\end{array}$ & Water related information & Downstream & National & Energy & 8,4 & 9,2 & 8,4 & 5 \\
\hline $\begin{array}{l}\text { Environmental Re- } \\
\text { search Institution }\end{array}$ & Environment & Other & Hydro-innovation & National & $\begin{array}{l}\text { Research and edu- } \\
\text { cation }\end{array}$ & 5,5 & 5,0 & 8,0 & 3 \\
\hline EU & Resource planning & Other & Downstream & Supranational & Other & 10,0 & 10,0 & 10,0 & 1 \\
\hline FEWSNET & Rescue and aid & Alert information & Downstream & Supranational & Humanitarian aid & 8,0 & 8,0 & 8,0 & 1 \\
\hline Firestone & $\begin{array}{l}\text { Economic service operations } \\
\text { and planning }\end{array}$ & Water related information & Downstream & National & Agriculture & 4,0 & 2,0 & 2,0 & 1 \\
\hline GWPIAO & Environment & Water related information & Hydro-innovation & Supranational & Water resources & 4,0 & 4,0 & 2,0 & 1 \\
\hline $\begin{array}{l}\text { Industry and Com- } \\
\text { merce Entities }\end{array}$ & $\begin{array}{l}\text { Economic service operations } \\
\text { and planning }\end{array}$ & Alert information & Downstream & Local & $\begin{array}{l}\text { Industry and Com- } \\
\text { merce }\end{array}$ & 6,0 & 6,0 & 3,0 & 3 \\
\hline IUCN & Environment & Water related information & Hydro-innovation & Supranational & Other & 4,0 & 6,0 & 2,0 & 1 \\
\hline $\begin{array}{l}\text { Local Administrative } \\
\text { Entity }\end{array}$ & Civil society & Alert information & Downstream & Local & Administration & 8,7 & 8,6 & 8,2 & 14 \\
\hline $\begin{array}{l}\text { Local Association for } \\
\text { Agriculture }\end{array}$ & $\begin{array}{l}\text { Economic service operations } \\
\text { and planning }\end{array}$ & Alert information & Downstream & Local & Agriculture & 5,7 & 3,0 & 4,9 & 9 \\
\hline $\begin{array}{l}\text { Local Entity for Civil } \\
\text { Security Enforcement }\end{array}$ & Rescue and aid & Alert information & Downstream & Local & Civil protection & 10,0 & 10,0 & 10,0 & 1 \\
\hline $\begin{array}{l}\text { Local Entity for Devel- } \\
\text { opment }\end{array}$ & Resource planning & Other & Downstream & Local & Development & 6,0 & 4,0 & 3,5 & 2 \\
\hline $\begin{array}{l}\text { Local Entity for Water } \\
\text { Resources Planning }\end{array}$ & Resource planning & Forecast refinement & Hydro-innovation & Local & Water resources & 4,3 & 3,8 & 7,3 & 4 \\
\hline Media & Civil society & Alert information & Downstream & NA & Other & 10,0 & 6,0 & 2,0 & 2 \\
\hline Metal Steel & $\begin{array}{l}\text { Economic service operations } \\
\text { and planning }\end{array}$ & Meteorological data & Downstream & National & $\begin{array}{l}\text { Industry and Com- } \\
\text { merce }\end{array}$ & 4,0 & 2,0 & 2,0 & 1 \\
\hline MNG & $\begin{array}{l}\text { Economic service operations } \\
\text { and planning }\end{array}$ & Meteorological data & Downstream & National & $\begin{array}{l}\text { Industry and Com- } \\
\text { merce }\end{array}$ & 4,0 & 2,0 & 2,0 & 1 \\
\hline $\begin{array}{l}\text { National Administra- } \\
\text { tive Entity }\end{array}$ & Civil society & Alert information & Downstream & National & Administration & 7,2 & 8,0 & 5,8 & 5 \\
\hline
\end{tabular}




\begin{tabular}{|c|c|c|c|c|c|c|c|c|c|}
\hline $\begin{array}{l}\text { Stakeholder } \\
{[1,9]}\end{array}$ & $\begin{array}{l}\text { Main interest } \\
{[3,11]}\end{array}$ & $\begin{array}{l}\text { Why use system } \\
{[4,12]}\end{array}$ & Information profile & Decisional level & Field & $\begin{array}{l}\text { Impt. } \\
{[6,14]}\end{array}$ & $\begin{array}{l}\text { Infl. } \\
{[7,15]}\end{array}$ & $\begin{array}{l}\text { Affect. } \\
{[8,16]}\end{array}$ & Count \\
\hline $\begin{array}{l}\text { National Agency for } \\
\text { Disaster Management } \\
\text { Planning }\end{array}$ & Disaster management & Alert information & Downstream & National & Civil protection & 8,7 & 8,4 & 7,3 & 12 \\
\hline $\begin{array}{l}\text { National Agency for } \\
\text { Meteorology }\end{array}$ & Technical & Meteorological data & Hydro-innovation & National & Meteorology & 10,0 & 9,2 & 6,7 & 7 \\
\hline $\begin{array}{l}\text { National Agency for } \\
\text { Water Resources }\end{array}$ & Resource planning & Forecast refinement & Hydro-innovation & National & Water resources & 7,0 & 8,3 & 6,5 & 8 \\
\hline $\begin{array}{l}\text { National Association } \\
\text { for Agriculture }\end{array}$ & $\begin{array}{l}\text { Economic service operations } \\
\text { and planning }\end{array}$ & Alert information & Downstream & National & Agriculture & 9,0 & 9,0 & 9,0 & 4 \\
\hline $\begin{array}{l}\text { National Entity for Aer- } \\
\text { ial Transportation }\end{array}$ & $\begin{array}{l}\text { Economic service operations } \\
\text { and planning }\end{array}$ & Meteorological data & Downstream & National & Transportation & 10,0 & 10,0 & 0,0 & 1 \\
\hline $\begin{array}{l}\text { National Entity for Ag- } \\
\text { riculture Management }\end{array}$ & $\begin{array}{l}\text { Economic service operations } \\
\text { and planning }\end{array}$ & Water related information & Downstream & National & Agriculture & 5,9 & 5,3 & 9,1 & 7 \\
\hline $\begin{array}{l}\text { National Entity for Civil } \\
\text { Security Enforcement }\end{array}$ & Rescue and aid & Alert information & Downstream & National & Civil protection & 8,0 & 8,1 & 6,9 & 7 \\
\hline $\begin{array}{l}\text { National Entity for De- } \\
\text { velopment }\end{array}$ & Resource planning & Alert information & Downstream & National & Development & 6,0 & 9,0 & 2,0 & 2 \\
\hline $\begin{array}{l}\text { National Entity for En- } \\
\text { ergy Planning }\end{array}$ & Resource planning & Alert information & Downstream & National & Energy & 10,0 & 10,0 & 10,0 & 1 \\
\hline $\begin{array}{l}\text { National Entity for } \\
\text { Transportation }\end{array}$ & $\begin{array}{l}\text { Economic service operations } \\
\text { and planning }\end{array}$ & Alert information & Downstream & National & Transportation & 10,0 & 10,0 & 10,0 & 1 \\
\hline $\begin{array}{l}\text { National Entity for Wa- } \\
\text { ter Infrastructure }\end{array}$ & Resource planning & Water related information & Hydro-innovation & National & Water services & 7,0 & 10,0 & 1,0 & 2 \\
\hline $\begin{array}{l}\text { National Entity for Wa- } \\
\text { terways Transport }\end{array}$ & $\begin{array}{l}\text { Economic service operations } \\
\text { and planning }\end{array}$ & Water related information & Downstream & National & Transportation & 6,3 & 5,5 & 6,5 & 4 \\
\hline $\begin{array}{l}\text { National Environment } \\
\text { Protection Entity }\end{array}$ & Environment & Water related information & Hydro-innovation & National & Other & 6,8 & 6,0 & 6,3 & 4 \\
\hline $\begin{array}{l}\text { National Governmen- } \\
\text { tal Entity for Agricul- } \\
\text { ture Planning }\end{array}$ & $\begin{array}{l}\text { Economic service operations } \\
\text { and planning }\end{array}$ & Alert information & Downstream & National & Agriculture & 7,3 & 6,0 & 7,3 & 7 \\
\hline $\begin{array}{l}\text { National Governmen- } \\
\text { tal Entity for Disaster }\end{array}$ & Disaster management & Alert information & Downstream & National & Civil protection & 8,9 & 9,1 & 7,0 & 9 \\
\hline
\end{tabular}




\begin{tabular}{|c|c|c|c|c|c|c|c|c|c|}
\hline $\begin{array}{l}\text { Stakeholder } \\
{[1,9]}\end{array}$ & $\begin{array}{l}\text { Main interest } \\
{[3,11]}\end{array}$ & $\begin{array}{l}\text { Why use system } \\
{[4,12]}\end{array}$ & Information profile & Decisional level & Field & $\begin{array}{l}\text { Impt. } \\
{[6,14]}\end{array}$ & $\begin{array}{l}\text { Infl. } \\
{[7,15]}\end{array}$ & $\begin{array}{l}\text { Affect. } \\
{[8,16]}\end{array}$ & Count \\
\hline \multicolumn{10}{|l|}{ Management Planning } \\
\hline $\begin{array}{l}\text { National Governmen- } \\
\text { tal Entity for Meteorol- } \\
\text { ogy }\end{array}$ & Technical & Meteorological data & Hydro-innovation & National & Meteorology & 10,0 & 9,3 & 9,0 & 3 \\
\hline $\begin{array}{l}\text { National Governmen- } \\
\text { tal Entity for Water Re- } \\
\text { sources }\end{array}$ & Resource planning & Forecast refinement & Hydro-innovation & National & Water resources & 9,6 & 9,4 & 8,5 & 14 \\
\hline $\begin{array}{l}\text { National Governmen- } \\
\text { tal Entity for Water } \\
\text { Services }\end{array}$ & $\begin{array}{l}\text { Economic service operations } \\
\text { and planning }\end{array}$ & Water related information & Downstream & National & Water services & 7,3 & 9,3 & 6,3 & 3 \\
\hline $\begin{array}{l}\text { National Health Ser- } \\
\text { vice Entity }\end{array}$ & Rescue and aid & Alert information & Downstream & National & Other & 0,0 & 0,0 & 10,0 & 1 \\
\hline $\begin{array}{l}\text { National Humanitarian } \\
\text { Aid Entity }\end{array}$ & Rescue and aid & Alert information & Downstream & National & Humanitarian aid & 6,0 & 4,0 & 4,0 & 1 \\
\hline NGO & Rescue and aid & Alert information & Downstream & NA & Humanitarian aid & 8,3 & 8,4 & 8,3 & 7 \\
\hline $\mathrm{OCHA}$ & Rescue and aid & Alert information & Downstream & Supranational & Humanitarian aid & 8,0 & 9,0 & 8,0 & 2 \\
\hline OMVG & Resource planning & Forecast refinement & Hydro-innovation & Supranational & Water resources & 8,0 & 8,0 & 9,3 & 4 \\
\hline OMVS & Resource planning & Forecast refinement & Hydro-innovation & Supranational & Water resources & 7,4 & 7,4 & 8,8 & 5 \\
\hline OXFAM & Rescue and aid & Alert information & Downstream & Supranational & Humanitarian aid & 6,0 & 6,0 & 5,0 & 2 \\
\hline PAM & Rescue and aid & Alert information & Downstream & Supranational & Humanitarian aid & 8,0 & 8,0 & 8,0 & 1 \\
\hline Red Cross & Rescue and aid & Alert information & Downstream & Supranational & Humanitarian aid & 7,5 & 6,5 & 5,4 & 8 \\
\hline $\begin{array}{l}\text { Regional Dam Man- } \\
\text { agement Entity }\end{array}$ & $\begin{array}{l}\text { Economic service operations } \\
\text { and planning }\end{array}$ & Water related information & Downstream & Supranational & Energy & 10,0 & 10,0 & 10,0 & 2 \\
\hline $\begin{array}{l}\text { Regional Entity for De- } \\
\text { velopment }\end{array}$ & Resource planning & Alert information & Downstream & Supranational & Development & 4,0 & 10,0 & 2,0 & 1 \\
\hline Research Institution & $\begin{array}{l}\text { Economic service operations } \\
\text { and planning }\end{array}$ & Other & Hydro-innovation & National & $\begin{array}{l}\text { Research and edu- } \\
\text { cation }\end{array}$ & 5,5 & 4,5 & 7,0 & 2 \\
\hline Statistics Institution & Technical & Other & Downstream & National & $\begin{array}{l}\text { Research and edu- } \\
\text { cation }\end{array}$ & 7,0 & 6,0 & 7,0 & 3 \\
\hline TOR & $\begin{array}{l}\text { Economic service operations } \\
\text { and planning }\end{array}$ & Meteorological data & Downstream & National & Energy & 4,0 & 0,0 & 0,0 & 1 \\
\hline
\end{tabular}




\begin{tabular}{|c|c|c|c|c|c|c|c|c|c|}
\hline $\begin{array}{l}\text { Stakeholder } \\
{[1,9]}\end{array}$ & $\begin{array}{l}\text { Main interest } \\
{[3,11]}\end{array}$ & $\begin{array}{l}\text { Why use system } \\
{[4,12]}\end{array}$ & Information profile & Decisional level & Field & $\begin{array}{l}\text { Impt. } \\
{[6,14]}\end{array}$ & $\begin{array}{l}\text { Infl. } \\
{[7,15]}\end{array}$ & $\begin{array}{l}\text { Affect. } \\
{[8,16]}\end{array}$ & Count \\
\hline Tullow & $\begin{array}{l}\text { Economic service operations } \\
\text { and planning }\end{array}$ & Meteorological data & Downstream & Supranational & Energy & 4,0 & 0,0 & 0,0 & 1 \\
\hline UN & Resource planning & Other & Downstream & Supranational & Other & 10,0 & 10,0 & 10,0 & 1 \\
\hline WASCAL & Environment & Alert information & Hydro-innovation & Supranational & $\begin{array}{l}\text { Research and edu- } \\
\text { cation }\end{array}$ & 4,0 & 4,0 & 2,0 & 1 \\
\hline $\begin{array}{l}\text { Water Resources De- } \\
\text { velopment Programs }\end{array}$ & Resource planning & Water related information & Downstream & National & Water resources & 4,0 & 8,5 & 3,0 & 3 \\
\hline Water Utility & $\begin{array}{l}\text { Economic service operations } \\
\text { and planning }\end{array}$ & Water related information & Downstream & National & Water services & 8,5 & 8,3 & 8,8 & 4 \\
\hline
\end{tabular}

\subsection{Objectives and attributes}

Table SI-5. Fundamental objectives and corresponding attributes, to quantify the system configurations performance, including short names, attribute unit, attribute range in parentheses (worst and best possible case), and short description of attribute.

\begin{tabular}{|c|c|c|c|c|c|c|}
\hline No. & $\begin{array}{l}\text { Higher level / lower } \\
\text { level objective full } \\
\text { name }\end{array}$ & $\begin{array}{l}\text { Short } \\
\text { objective } \\
\text { name }\end{array}$ & $\begin{array}{l}\text { Attribute full } \\
\text { name }\end{array}$ & $\begin{array}{l}\text { Short attribute } \\
\text { name }\end{array}$ & $\begin{array}{l}\text { Unit } \\
\text { (range) }\end{array}$ & Attribute description \\
\hline 1 & $\begin{array}{l}\text { High information accu- } \\
\text { racy and clarity }\end{array}$ & 1_accuracy & & & & \\
\hline 1.1 & $\begin{array}{l}\text { High accuracy of infor- } \\
\text { mation }\end{array}$ & 11_accur_info & $\begin{array}{l}\text { Value of KGE index for } \\
1,3,10 \text { day forecasts }\end{array}$ & 11_kge & $\begin{array}{l}\text { Value } \\
(0-1)\end{array}$ & $\begin{array}{l}\text { Level of accuracy consisting of KGE for } 1,3 \text {, and } 10 \text { day forecasts; index } \\
\text { transformed into [0:1] value by aggregation with a weighted sum, using differ- } \\
\text { ent weights for the sub-attributes. }\end{array}$ \\
\hline 1.2 & $\begin{array}{l}\text { Clear flood risk infor- } \\
\text { mation }\end{array}$ & 12_clear_info & $\begin{array}{l}\text { Clarity of flood risk } \\
\text { thresholds in risk man- } \\
\text { agement }\end{array}$ & 12_info & $\begin{array}{l}\text { Value } \\
(0-1)\end{array}$ & $\begin{array}{l}\text { Levels of flood risk threshold of three categorical sub-attributes: 1) location, } \\
\text { 2) possibility to calculate risk, and 3) ease of applying to flood risk manage- } \\
\text { ment; aggregated into [0:1] value, using an equally weighted sum. }\end{array}$ \\
\hline 2 & $\begin{array}{l}\text { Good information ac- } \\
\text { cess }\end{array}$ & 2_access & & & & \\
\hline 2.1 & $\begin{array}{l}\text { Reliable access to infor- } \\
\text { mation }\end{array}$ & 21_reliable_info & $\begin{array}{l}\text { Stability of distribution } \\
\text { channel }\end{array}$ & 21_channel & $\begin{array}{l}\text { Score } \\
(0-2.2)\end{array}$ & $\begin{array}{l}\text { Access to information calculated by the weighted sum of categorical stability } \\
\text { of access (i.e., stable, sporadic, none) for each distribution channel (i.e., } \\
\text { SMS, Email, fanfar.eu). }\end{array}$ \\
\hline
\end{tabular}




\begin{tabular}{|c|c|c|c|c|c|c|}
\hline No. & $\begin{array}{l}\text { Higher level / lower } \\
\text { level objective full } \\
\text { name }\end{array}$ & $\begin{array}{l}\text { Short } \\
\text { objective } \\
\text { name }\end{array}$ & $\begin{array}{l}\text { Attribute full } \\
\text { name }\end{array}$ & $\begin{array}{l}\text { Short attribute } \\
\text { name }\end{array}$ & $\begin{array}{l}\text { Unit } \\
\text { (range) }\end{array}$ & Attribute description \\
\hline 2.2 & $\begin{array}{l}\text { Timely production, distri- } \\
\text { bution, and access to } \\
\text { info }\end{array}$ & 22_timely_info & $\begin{array}{l}\text { Time to produce, distrib- } \\
\text { ute, and access infor- } \\
\text { mation }\end{array}$ & 22_time & $\begin{array}{l}\text { Value } \\
(0-1)\end{array}$ & $\begin{array}{l}\text { Time required to produce forecast, access distribution channels, and access } \\
\text { IVP; transformed to [0:1] value with linear interpolation, aggregated with } \\
\text { equally weighted sum. }\end{array}$ \\
\hline 2.3 & Several languages & 23_language & $\begin{array}{l}\text { Number of languages } \\
\text { available for system } \\
\text { components }\end{array}$ & 23_langue & $\begin{array}{l}\text { Score }(0- \\
17.64)\end{array}$ & $\begin{array}{l}\text { Number of languages (En, Fr, Pt, Ara) for different system components and } \\
\text { channels with different weights for languages and system components; } \\
\text { weighted sum resulting in a score. }\end{array}$ \\
\hline 3 & Low costs & 3_costs & & & & \\
\hline 3.1 & Short development time & 31_develop_time & $\begin{array}{l}\text { Time required to de- } \\
\text { velop and implement } \\
\text { system components }\end{array}$ & 31_devlptime & $\begin{array}{l}\text { Days } \\
(0-1095)\end{array}$ & $\begin{array}{l}\text { Total time needed for the development and implementation of all technical } \\
\text { system components in days. }\end{array}$ \\
\hline 3.2 & Low operation costs & 32_costs & $\begin{array}{l}\text { Annual operation and } \\
\text { maintenance costs }\end{array}$ & 32_costs & $\begin{array}{l}\text { Value } \\
(0-1)\end{array}$ & $\begin{array}{l}\text { Annual costs }(€ / \text { year) to operate and maintain the system for entire West Af- } \\
\text { rica; estimated costs based on operation and maintenance of individual sys- } \\
\text { tem components included in each configuration; transformed into [0:1] value. }\end{array}$ \\
\hline 4 & High sustainability & 4_sustainable & & & & \\
\hline 4.1 & $\begin{array}{l}\text { Long-term financing se- } \\
\text { cured }\end{array}$ & 41_sust_financing & $\begin{array}{l}\text { Secured financing be- } \\
\text { yond } 2020\end{array}$ & 41_finance & $\begin{array}{l}\text { Value } \\
(0-1)\end{array}$ & $\begin{array}{l}\text { Level of secured financing beyond } 2020 \text { consisting of two sub-attributes } \\
\text { (costs covered, and duration of financing) transformed to [0:1] value, aggre- } \\
\text { gated with equally weighed sum. }\end{array}$ \\
\hline 4.2 & $\begin{array}{l}\text { Skillful human resources } \\
\text { available }\end{array}$ & 42_human_resour & $\begin{array}{l}\text { Number of people in W } \\
\text { Africa for development, } \\
\text { O\&M, and access \& in- } \\
\text { terpretation }\end{array}$ & 42_experts & $\begin{array}{l}\text { Value } \\
(0-1)\end{array}$ & $\begin{array}{l}\text { Skillful human resources available, based on three sub-attributes (number of } \\
\text { people in West Africa available to 1) develop \& maintain, 2) operate, and 3) } \\
\text { access \& interpret the system) transformed to a [0:1] value, aggregated with } \\
\text { equally weighted sum. }\end{array}$ \\
\hline 4.3 & Good support system & 43_support_syst & $\begin{array}{l}\text { Information quantity in } \\
\text { support system and time } \\
\text { of response to resolve } \\
\text { issues }\end{array}$ & 43_suppsys & $\begin{array}{l}\text { Value } \\
(0-1)\end{array}$ & $\begin{array}{l}\text { Support system quality consisting of two sub-attributes: 1) information quan- } \\
\text { tity, and 2) time for response of support system; transformed into a [0:1] } \\
\text { value, aggregated with equally weighted sum. }\end{array}$ \\
\hline
\end{tabular}




\subsection{FANFAR system configurations}

The FANFAR system configurations, were generated using the Strategy Generation Table method, and Brainwriting 635 combined with Cadavre Exquis. The system configurations are combinations of different system characteristics. These were generated separately for the Hydrology-TEP (Table SI-6), and the Interactive Visualization Portal (IVP; Table SI-7). The latter is the interface of FANFAR, with which most users will normally interact. In the FANFAR co-design workshops, all emergency managers interacted with the IVP, and most of the hydrologists that need the FANFAR system to create forecasts and alerts. The Hydrology-TEP, i.e., the actual forecast production system, was only accessed by very experienced hydrologists, which in the co-design workshops were members of the FANFAR consortium from AGRHYMET.

Table SI-6. Characteristics of the system configurations for the Hydrology-TEP (development stage summer 2019). The columns display the key components of the FANFAR system with relevance for Hydrology-TEP including hydrological observation data types, meteorological analysis and forecast data, hydrological model (WWH: World-Wide HYPE: Arheimer et al., 2020) (Niger HYPE: Andersson et al., 2017), forecasted output variables, distribution options of derived information (forecasts and alerts), degree of automatization of information distribution, language, and support system. In situ: local hydrometric gauge observation data; EO: Earth Observations; HydroGFD: Hydrological Global Forcing Data (merged data set of historical precipitation and temperature from meteorological reanalysis and global observations; Berg et al., 2020;Berg et al., 2018); HydroGFD-West Africa from AGRHYMET: HydroGFD2 adjusted by West African meteorological observations; GFS: Global Forecast System (https://www.ncdc.noaa.gov/); ECMWF: European Centre for Medium-Range Weather Forecasts adjusted by West African meteorological observations; GFS: Global Forecast System (htt
(https://www.ecmwf.int/en/forecasts); En: English; Fr: French; Pt: Portuguese; Ar: Arabic.

\begin{tabular}{|c|c|c|c|c|c|c|c|c|c|}
\hline Code & $\begin{array}{l}\text { System Con- } \\
\text { figuration }\end{array}$ & $\begin{array}{l}\text { Hydrologi- } \\
\text { cal obser- } \\
\text { vation } \\
\text { types }\end{array}$ & $\begin{array}{l}\text { Meteorological in- } \\
\text { put/forcing data }\end{array}$ & $\begin{array}{l}\text { Hydrological } \\
\text { models }\end{array}$ & $\begin{array}{l}\text { Forecast output varia- } \\
\text { bles }\end{array}$ & $\begin{array}{l}\text { Distribution configurations } \\
\text { of derived information } \\
\text { (forecasts and alerts) }\end{array}$ & $\begin{array}{l}\text { Automatization } \\
\text { (of information } \\
\text { distribution) }\end{array}$ & Language & Support system \\
\hline $\begin{array}{l}\text { a_Fast- } \\
\text { dev }\end{array}$ & $\begin{array}{l}\text { Least re- } \\
\text { sources for } \\
\text { development }\end{array}$ & None & $\begin{array}{l}\text { Current data from } \\
\text { SMHI } \\
\text { (HydroGFD v2) }\end{array}$ & Niger HYPE & Streamflow & Web visualization & $\begin{array}{l}\text { Automatic push of } \\
\text { data to distribution } \\
\text { channels }\end{array}$ & En & $\begin{array}{l}\text { - Forum; } \\
\text { - Knowledge base; } \\
\text { - Help desk }\end{array}$ \\
\hline $\begin{array}{l}\text { b_Res- } \\
\text { user }\end{array}$ & $\begin{array}{l}\text { Least re- } \\
\text { sources for } \\
\text { users }\end{array}$ & $\begin{array}{l}\text { In situ } \\
\text { country } \\
\text { level data: } \\
\text { water level, } \\
\text { discharge }\end{array}$ & $\begin{array}{l}\text { Improved data from } \\
\text { SMHI } \\
\text { (HydroGFD v3) }\end{array}$ & WWH & $\begin{array}{l}\text { - Streamflow; } \\
\text { - Water level; } \\
\text { - Precipitation; } \\
\text { - Evaporation; } \\
\text { - Soil moisture }\end{array}$ & $\begin{array}{l}\text { - Web visualization; } \\
\text { - Login to H-TEP } \\
\text { - SMS alert } \\
\text { - Email alert; } \\
\text { - WhatsApp alert; } \\
\text { - Radio, TV; } \\
\text { - Traditional word of mouth }\end{array}$ & $\begin{array}{l}\text { Automatic pro- } \\
\text { cessing with man- } \\
\text { ual control of dis- } \\
\text { tribution by opera- } \\
\text { tor }\end{array}$ & $\begin{array}{l}\text { - En; } \\
\text { - } \mathrm{Fr} \\
\text { - } \mathrm{Pt} \\
\text { - } \mathrm{Ar}\end{array}$ & $\begin{array}{l}\text { - Forum } \\
\text { - Knowledge base }\end{array}$ \\
\hline $\begin{array}{l}\text { c_Easy- } \\
\text { use }\end{array}$ & $\begin{array}{l}\text { Most easy-to } \\
\text { use }\end{array}$ & $\begin{array}{l}\text { EO: water } \\
\text { level }\end{array}$ & $\begin{array}{l}\text { Current data from } \\
\text { SMHI } \\
\text { (HydroGFD v2) }\end{array}$ & Niger HYPE & Water level & $\begin{array}{l}\text { - Web visualization; } \\
\text { - SMS alert; } \\
\text { - WhatsApp alert; } \\
\text { - Radio, TV; } \\
\text { - Traditional word of mouth }\end{array}$ & $\begin{array}{l}\text { Automatic push of } \\
\text { data to distribution } \\
\text { channels }\end{array}$ & $\begin{array}{l}\text { - En; } \\
\text { - } \mathrm{Fr} \\
\text { - } \mathrm{Pt} \\
\text { - } \mathrm{Ar}\end{array}$ & $\begin{array}{l}\text { - Forum } \\
\text { - Knowledge base }\end{array}$ \\
\hline
\end{tabular}




\begin{tabular}{|c|c|c|c|c|c|c|c|c|c|}
\hline Code & $\begin{array}{l}\text { System Con- } \\
\text { figuration }\end{array}$ & $\begin{array}{l}\text { Hydrologi- } \\
\text { cal obser- } \\
\text { vation } \\
\text { types }\end{array}$ & $\begin{array}{l}\text { Meteorological in- } \\
\text { put/forcing data }\end{array}$ & $\begin{array}{l}\text { Hydrological } \\
\text { models }\end{array}$ & $\begin{array}{l}\text { Forecast output varia- } \\
\text { bles }\end{array}$ & $\begin{array}{l}\text { Distribution configurations } \\
\text { of derived information } \\
\text { (forecasts and alerts) }\end{array}$ & $\begin{array}{l}\text { Automatization } \\
\text { (of information } \\
\text { distribution) }\end{array}$ & Language & Support system \\
\hline d_Fast & $\begin{array}{l}\text { Fastest sys- } \\
\text { tem }\end{array}$ & $\begin{array}{l}\text { EO: water } \\
\text { level }\end{array}$ & $\begin{array}{l}\text { Current data from } \\
\text { SMHI } \\
\text { (HydroGFD v2) }\end{array}$ & Niger HYPE & Water level & $\begin{array}{l}\text { - Web visualization; } \\
\text { - SMS automatic push of } \\
\text { data; } \\
\text { - SMS alert } \\
\text { - Email alert; } \\
\text { - WhatsApp alert; } \\
\text { - Radio, TV; } \\
\text { - Traditional word of mouth }\end{array}$ & $\begin{array}{l}\text { Automatic push of } \\
\text { data to distribution } \\
\text { channels }\end{array}$ & En & $\begin{array}{l}\text { - Forum } \\
\text { - Knowledge base; } \\
\text { - Help desk; } \\
\text { - Demos, tutorials; } \\
\text { - SMS, Email; } \\
\text { - Phone }\end{array}$ \\
\hline $\begin{array}{l}\text { e_Con- } \\
\text { sent }\end{array}$ & $\begin{array}{l}\text { Highest con- } \\
\text { sensus }\end{array}$ & $\begin{array}{l}\text { System can } \\
\text { handle all } \\
\text { types of } \\
\text { data: in } \\
\text { situ, EO }\end{array}$ & $\begin{array}{l}\text { - American meteo } \\
\text { (e.g. GFS); } \\
\text { - ECMWF ensem- } \\
\text { ble forecasts; } \\
\text { - HydroGFD-West } \\
\text { Africa from } \\
\text { AGRHYMET }\end{array}$ & $\begin{array}{l}\text { - Niger HYPE; } \\
\text { - WWH }\end{array}$ & $\begin{array}{l}\text { - Streamflow; } \\
\text { - Water level; } \\
\text { - Precipitation; } \\
\text { - Evaporation; } \\
\text { - Soil moisture }\end{array}$ & $\begin{array}{l}\text { - Web visualization; } \\
\text { - Login to H-TEP; } \\
\text { - SMS alert; } \\
\text { - Email alert; } \\
\text { - WhatsApp alert; } \\
\text { - Radio, TV; } \\
\text { - Traditional word of mouth }\end{array}$ & $\begin{array}{l}\text { Automatic pro- } \\
\text { cessing with man- } \\
\text { ual control of dis- } \\
\text { tribution by opera- } \\
\text { tor }\end{array}$ & $\begin{array}{l}\text { - En; } \\
\text { - Fr; } \\
\text { - Pt; } \\
\text { - Ar }\end{array}$ & $\begin{array}{l}\text { - Forum } \\
\text { - Knowledge base; } \\
\text { - Help desk; } \\
\text { - Demos, tutorials; } \\
\text { - SMS, Email; } \\
\text { - Phone }\end{array}$ \\
\hline f_Robust & Most robust & $\begin{array}{l}\text { EO: water } \\
\text { level }\end{array}$ & $\begin{array}{l}\text { Current data from } \\
\text { SMHI } \\
\text { (HydroGFD v2) }\end{array}$ & $\begin{array}{l}\text { - Niger HYPE; } \\
\text { - WWH (or } \\
\text { WWH alone) }\end{array}$ & $\begin{array}{l}\text { - Streamflow; } \\
\text { - Water level; } \\
\text { - Precipitation; } \\
\text { - Evaporation; } \\
\text { - Soil moisture }\end{array}$ & $\begin{array}{l}\text { - Web visualization; } \\
\text { - Login to H-TEP; } \\
\text { - SMS alert; } \\
\text { - Email alert; } \\
\text { - WhatsApp alert; } \\
\text { - Radio, TV; } \\
\text { - Traditional word of mouth }\end{array}$ & $\begin{array}{l}\text { Automatic pro- } \\
\text { cessing with man- } \\
\text { ual control of dis- } \\
\text { tribution by opera- } \\
\text { tor }\end{array}$ & $\begin{array}{l}\text { - En; } \\
\text { - } \mathrm{Fr} \\
\text { - } \mathrm{Pt} \\
\text { - } \mathrm{Ar}\end{array}$ & $\begin{array}{l}\text { - Forum } \\
\text { - Knowledge base; } \\
\text { - Help desk; } \\
\text { - Demos, tutorials; } \\
\text { - SMS, Email; } \\
\text { - Phone }\end{array}$ \\
\hline $\begin{array}{l}\text { g_At- } \\
\text { tractve }\end{array}$ & $\begin{array}{l}\text { Most attrac- } \\
\text { tive }\end{array}$ & $\begin{array}{l}\text { System can } \\
\text { handle all } \\
\text { types of } \\
\text { data: in } \\
\text { situ, EO }\end{array}$ & $\begin{array}{l}\text { - American meteo } \\
\text { (e.g. GFS); } \\
\text { - ECMWF ensem- } \\
\text { ble forecasts; } \\
\text { - HydroGFD-West } \\
\text { Africa from } \\
\text { AGRHYMET }\end{array}$ & $\begin{array}{l}\text { - Niger- } \\
\text { HYPE; } \\
\text { - WWH }\end{array}$ & $\begin{array}{l}\text { - Streamflow; } \\
\text { - Water level; } \\
\text { - Precipitation; } \\
\text { - Evaporation; } \\
\text { - Soil moisture }\end{array}$ & $\begin{array}{l}\text { - Web visualization; } \\
\text { - Login to H-TEP; } \\
\text { - SMS alert; } \\
\text { - Email alert; } \\
\text { - WhatsApp alert; } \\
\text { - Radio, TV; } \\
\text { - Traditional word of mouth }\end{array}$ & $\begin{array}{l}\text { Automatic pro- } \\
\text { cessing with man- } \\
\text { ual control of dis- } \\
\text { tribution by opera- } \\
\text { tor }\end{array}$ & $\begin{array}{l}\text { - En; } \\
\text { - } \mathrm{Fr} \\
\text { - } \mathrm{Pt} \\
\text { - } \mathrm{Ar}\end{array}$ & $\begin{array}{l}\text { - Forum } \\
\text { - Knowledge base; } \\
\text { - Help desk; } \\
\text { - Demos, tutorials; } \\
\text { - SMS, Email; } \\
\text { - Phone }\end{array}$ \\
\hline h_Equipp & $\begin{array}{l}\text { Fully } \\
\text { equipped }\end{array}$ & $\begin{array}{l}\text { System can } \\
\text { handle all } \\
\text { types of }\end{array}$ & $\begin{array}{l}\text { - American meteo } \\
\text { (e.g. GFS); }\end{array}$ & $\begin{array}{l}\text { - Niger HYPE; } \\
\text { - WWH }\end{array}$ & $\begin{array}{l}\text { - Streamflow; } \\
\text { - Water level; }\end{array}$ & $\begin{array}{l}\text { - Web visualization; } \\
\text { - Login to H-TEP; }\end{array}$ & $\begin{array}{l}\text { Automatic pro- } \\
\text { cessing, with pos- } \\
\text { sibility to choose }\end{array}$ & $\begin{array}{l}\text { - En; } \\
\text { - Fr; }\end{array}$ & $\begin{array}{l}\text { - Forum } \\
\text { - Knowledge base; }\end{array}$ \\
\hline
\end{tabular}




\begin{tabular}{|c|c|c|c|c|c|c|c|c|c|}
\hline Code & $\begin{array}{l}\text { System Con- } \\
\text { figuration }\end{array}$ & $\begin{array}{l}\text { Hydrologi- } \\
\text { cal obser- } \\
\text { vation } \\
\text { types }\end{array}$ & $\begin{array}{l}\text { Meteorological in- } \\
\text { put/forcing data }\end{array}$ & $\begin{array}{l}\text { Hydrological } \\
\text { models }\end{array}$ & $\begin{array}{l}\text { Forecast output varia- } \\
\text { bles }\end{array}$ & $\begin{array}{l}\text { Distribution configurations } \\
\text { of derived information } \\
\text { (forecasts and alerts) }\end{array}$ & $\begin{array}{l}\text { Automatization } \\
\text { (of information } \\
\text { distribution) }\end{array}$ & Language & Support system \\
\hline & & $\begin{array}{l}\text { data: in } \\
\text { situ, EO }\end{array}$ & $\begin{array}{l}\text { - ECMWF ensem- } \\
\text { ble forecasts; } \\
\text { - HydroGFD-West } \\
\text { Africa from } \\
\text { AGRHYMET }\end{array}$ & & $\begin{array}{l}\text { - Precipitation; } \\
\text { - Evaporation; } \\
\text { - Soil moisture }\end{array}$ & $\begin{array}{l}\text { - FANFAR and national } \\
\text { FTP; } \\
\text { - API; } \\
\text { - SMS alert; } \\
\text { - Email alert; } \\
\text { - WhatsApp alert; } \\
\text { - Radio, TV; } \\
\text { - Traditional word of mouth }\end{array}$ & $\begin{array}{l}\text { automatic and } \\
\text { manual control of } \\
\text { distribution by op- } \\
\text { erator }\end{array}$ & $\begin{array}{l}\text { - } \mathrm{Pt} \\
\text { - } \mathrm{Ar}\end{array}$ & $\begin{array}{l}\text { - Help desk; } \\
\text { - Demos, tutorials; } \\
\text { - SMS, Email; } \\
\text { - Phone }\end{array}$ \\
\hline i_Calibr & $\begin{array}{l}\text { Recalibrated } \\
\text { HYPE models }\end{array}$ & None & $\begin{array}{l}\text { Current data from } \\
\text { SMHI } \\
\text { (HydroGFD v2) }\end{array}$ & $\begin{array}{l}\text { WWH cali- } \\
\text { brated for West } \\
\text { Africa }\end{array}$ & $\begin{array}{l}\text { - Streamflow; } \\
\text { - Water level; } \\
\text { - Precipitation; } \\
\text { - Evaporation; } \\
\text { - Soil moisture }\end{array}$ & $\begin{array}{l}\text { - Web visualization; } \\
\text { - Login to H-TEP; } \\
\text { - SMS alert; } \\
\text { - Email alert; } \\
\text { - WhatsApp alert; } \\
\text { - Radio, TV; } \\
\text { - Traditional word of mouth }\end{array}$ & $\begin{array}{l}\text { Automatic pro- } \\
\text { cessing with man- } \\
\text { ual control of dis- } \\
\text { tribution by opera- } \\
\text { tor }\end{array}$ & $\begin{array}{l}\text { - En; } \\
\text { - Fr; } \\
\text { - Pt }\end{array}$ & $\begin{array}{l}\text { - Forum; } \\
\text { - Knowledge base; } \\
\text { - Help desk }\end{array}$ \\
\hline j_Cal-EO & $\begin{array}{l}\text { Recalibrated } \\
\text { HYPE models } \\
\text { and EO data }\end{array}$ & $\begin{array}{l}\text { EO: water } \\
\text { level }\end{array}$ & $\begin{array}{l}\text { Current data from } \\
\text { SMHI } \\
\text { (HydroGFD v2) }\end{array}$ & $\begin{array}{l}\text { WWH cali- } \\
\text { brated for West } \\
\text { Africa }\end{array}$ & $\begin{array}{l}\text { - Streamflow; } \\
\text { - Water level; } \\
\text { - Precipitation; } \\
\text { - Evaporation; } \\
\text { - Soil moisture }\end{array}$ & $\begin{array}{l}\text { - Web visualization; } \\
\text { - Login to H-TEP; } \\
\text { - SMS alert; } \\
\text { - Email alert; } \\
\text { - WhatsApp alert; } \\
\text { - Radio, TV; } \\
\text { - Traditional word of mouth }\end{array}$ & $\begin{array}{l}\text { Automatic pro- } \\
\text { cessing with man- } \\
\text { ual control of dis- } \\
\text { tribution by opera- } \\
\text { tor }\end{array}$ & $\begin{array}{l}\text { - En; } \\
\text { - Fr; } \\
\text { - } \mathrm{Pt}\end{array}$ & $\begin{array}{l}\text { - Forum; } \\
\text { - Knowledge base; } \\
\text { - Help desk }\end{array}$ \\
\hline $\begin{array}{l}\text { k_Cal- } \\
\text { EO-situ }\end{array}$ & $\begin{array}{l}\text { Recalibrated } \\
\text { HYPE models } \\
\text { and EO data }\end{array}$ & $\begin{array}{l}\text { System can } \\
\text { handle all } \\
\text { types of } \\
\text { data: in } \\
\text { situ, EO }\end{array}$ & $\begin{array}{l}\text { Current data from } \\
\text { SMHI } \\
\text { (HydroGFD v2) }\end{array}$ & $\begin{array}{l}\text { WWH cali- } \\
\text { brated for West } \\
\text { Africa }\end{array}$ & $\begin{array}{l}\text { - Streamflow; } \\
\text { - Water level; } \\
\text { - Precipitation; } \\
\text { - Evaporation; } \\
\text { - Soil moisture }\end{array}$ & $\begin{array}{l}\text { - Web visualization; } \\
\text { - Login to H-TEP; } \\
\text { - SMS alert; } \\
\text { - Email alert; } \\
\text { - WhatsApp alert; } \\
\text { - Radio, TV; } \\
\text { - Traditional word of mouth }\end{array}$ & $\begin{array}{l}\text { Automatic pro- } \\
\text { cessing with man- } \\
\text { ual control of dis- } \\
\text { tribution by opera- } \\
\text { tor }\end{array}$ & $\begin{array}{l}\text { - En; } \\
\text { - Fr; } \\
\text { - Pt }\end{array}$ & $\begin{array}{l}\text { - Forum; } \\
\text { - Knowledge base; } \\
\text { - Help desk }\end{array}$ \\
\hline
\end{tabular}


Table SI-7. Characteristics of the system configurations for the Interactive Visualization Portal (IVP; development stage summer 2019). Columns indicate observed variable, displayed model performance (accuracy of forecast), forecasted variable, flood hazard reference threshold type, data download, interactivity, language, and alert notification system. NSE: NashSutcliffe Efficiency; KGE: Kling-Gupta Efficiency; En: English; Fr: French; Pt: Portuguese; Ar: Arabic.

\begin{tabular}{|c|c|c|c|c|c|c|c|c|c|}
\hline Code & $\begin{array}{l}\text { System } \\
\text { Configura- } \\
\text { tion }\end{array}$ & $\begin{array}{l}\text { Observed } \\
\text { variable }\end{array}$ & $\begin{array}{l}\text { Model performance, } \\
\text { accuracy }\end{array}$ & $\begin{array}{l}\text { Forecasted varia- } \\
\text { ble }\end{array}$ & $\begin{array}{l}\text { Flood hazard refer- } \\
\text { ence threshold type }\end{array}$ & Data download & Interactivity & $\begin{array}{l}\text { Lan- } \\
\text { guage }\end{array}$ & $\begin{array}{l}\text { Alert notification to operating } \\
\text { agencies and stakeholders }\end{array}$ \\
\hline $\begin{array}{l}\text { a_Fast- } \\
\text { dev }\end{array}$ & $\begin{array}{l}\text { Least re- } \\
\text { sources for } \\
\text { development }\end{array}$ & None & $\begin{array}{l}\text { No performance metrics } \\
\text { shown }\end{array}$ & River discharge & $\begin{array}{l}\text { Return periods (simu- } \\
\text { lation) }\end{array}$ & None & $\begin{array}{l}\text { Interactive } \\
\text { website } \\
\text { (zoom, click, } \\
\text { etc.) }\end{array}$ & En & None \\
\hline $\begin{array}{l}\text { b_Res- } \\
\text { user }\end{array}$ & $\begin{array}{l}\text { Least re- } \\
\text { sources for } \\
\text { users }\end{array}$ & $\begin{array}{l}\text { In situ: water } \\
\text { level }\end{array}$ & $\begin{array}{l}\text { No performance metrics } \\
\text { shown }\end{array}$ & River discharge & $\begin{array}{l}\text { - Return periods } \\
\text { (simulation); } \\
\text { - Return periods (ob- } \\
\text { servations; only } \\
\text { stations); } \\
\text { - Selected historic } \\
\text { years (e.g., 2012); } \\
\text { - User defined } \\
\text { thresholds for spe- } \\
\text { cific location }\end{array}$ & $\begin{array}{l}\text { - Excel table for se- } \\
\text { lected station; } \\
\text { - Map of displayed var- } \\
\text { iables (PNG); } \\
\text { - Map of variables } \\
\text { (Shapefile); } \\
\text { - Charts, Graphs (e.g., } \\
\text { histogram, pie, bars) }\end{array}$ & $\begin{array}{l}\text { Interactive } \\
\text { website } \\
\text { (zoom, click, } \\
\text { etc.) }\end{array}$ & $\begin{array}{l}\text { - En; } \\
\text { - } \mathrm{Fr} \\
\text { - } \mathrm{Pt} \\
\text { - } \mathrm{Ar}\end{array}$ & $\begin{array}{l}\text { - Automatic alerts to agencies, } \\
\text { that can forward alerts to } \\
\text { stakeholders via existing dis- } \\
\text { tribution channels; } \\
\text { - Automatic alerts to agencies } \\
\text { that can authorize system to } \\
\text { forward alert to stakeholders }\end{array}$ \\
\hline $\begin{array}{l}\text { c_Easy- } \\
\text { use }\end{array}$ & $\begin{array}{l}\text { Most easy to } \\
\text { use }\end{array}$ & $\begin{array}{l}\text { - In situ: wa- } \\
\text { ter level; } \\
\text { - EO: water } \\
\text { level }\end{array}$ & $\begin{array}{l}\text { Performance metric } \\
\text { shown (e.g., NSE, KGE; } \\
\text { with colored levels) }\end{array}$ & $\begin{array}{l}\text { - River discharge; } \\
\text { - Water level; } \\
\text { - Precipitation; } \\
\text { - Evaporation }\end{array}$ & $\begin{array}{l}\text { - Return periods } \\
\text { (simulation); } \\
\text { - Selected historic } \\
\text { years (e.g., 2012) }\end{array}$ & $\begin{array}{l}\text { - Excel table for se- } \\
\text { lected station; } \\
\text { - Charts, Graphs (e.g., } \\
\text { histogram, pie, bars) }\end{array}$ & $\begin{array}{l}\text { Website with } \\
\text { static images } \\
\text { (no zoom, no } \\
\text { click) }\end{array}$ & $\begin{array}{l}\text { - En; } \\
\text { - Fr; } \\
\text { - Pt }\end{array}$ & $\begin{array}{l}\text { - Automatic alerts to agencies } \\
\text { - Automatic alerts sent directly } \\
\text { to stakeholders }\end{array}$ \\
\hline d_Fast & $\begin{array}{l}\text { Fastest sys- } \\
\text { tem }\end{array}$ & None & $\begin{array}{l}\text { No performance metrics } \\
\text { shown }\end{array}$ & River discharge & $\begin{array}{l}\text { Return periods (simu- } \\
\text { lation) }\end{array}$ & None & $\begin{array}{l}\text { Website with } \\
\text { static images } \\
\text { (no zoom, no } \\
\text { click) }\end{array}$ & $\mathrm{En}$ & $\begin{array}{l}\text { - Automatic alerts to agencies } \\
\text { to stakeholders }\end{array}$ \\
\hline $\begin{array}{l}\text { e_Con- } \\
\text { sent }\end{array}$ & $\begin{array}{l}\text { Highest con- } \\
\text { sensus }\end{array}$ & $\begin{array}{l}\text { - In situ: wa- } \\
\text { ter level, } \\
\text { river dis- } \\
\text { charge; } \\
\text { - EO: water } \\
\text { level, pre- } \\
\text { cipitation }\end{array}$ & $\begin{array}{l}\text { - Performance metric } \\
\text { shown (e.g., NSE, } \\
\text { KGE; with colored } \\
\text { levels) } \\
\text { - Blank areas with too } \\
\text { low forecast perfor- } \\
\text { mance (based on } \\
\text { NSE, KGE) }\end{array}$ & $\begin{array}{l}\text { - River discharge; } \\
\text { - Water level; } \\
\text { - Precipitation; } \\
\text { - Evaporation }\end{array}$ & $\begin{array}{l}\text { - Return periods } \\
\text { (simulation); } \\
\text { - Selected historic } \\
\text { years (e.g., 2012) }\end{array}$ & $\begin{array}{l}\text { - Excel table for se- } \\
\text { lected station; } \\
\text { - Map of displayed var- } \\
\text { iables (PNG); } \\
\text { - Map of variables } \\
\text { (Shapefile); } \\
\text { - Charts, Graphs (e.g., } \\
\text { histogram, pie, bars) }\end{array}$ & $\begin{array}{l}\text { Interactive } \\
\text { website } \\
\text { (zoom, click, } \\
\text { etc.) }\end{array}$ & $\begin{array}{l}\text { - En; } \\
\text { - Fr; } \\
\text { - Pt }\end{array}$ & $\begin{array}{l}\text { - Automatic alerts to agencies, } \\
\text { that can forward alerts to } \\
\text { stakeholders via existing dis- } \\
\text { tribution channels; } \\
\text { - Automatic alerts to agencies } \\
\text { that can authorize system to } \\
\text { forward alert to stakeholders }\end{array}$ \\
\hline
\end{tabular}




\begin{tabular}{|c|c|c|c|c|c|c|c|c|c|}
\hline Code & $\begin{array}{l}\text { System } \\
\text { Configura- } \\
\text { tion }\end{array}$ & $\begin{array}{l}\text { Observed } \\
\text { variable }\end{array}$ & $\begin{array}{l}\text { Model performance, } \\
\text { accuracy }\end{array}$ & $\begin{array}{l}\text { Forecasted varia- } \\
\text { ble }\end{array}$ & $\begin{array}{l}\text { Flood hazard refer- } \\
\text { ence threshold type }\end{array}$ & Data download & Interactivity & $\begin{array}{l}\text { Lan- } \\
\text { guage }\end{array}$ & $\begin{array}{l}\text { Alert notification to operating } \\
\text { agencies and stakeholders }\end{array}$ \\
\hline f_Robust & Most robust & $\begin{array}{l}\text { - In situ: wa- } \\
\text { ter level, } \\
\text { river dis- } \\
\text { charge; } \\
\text { - EO: water } \\
\text { level, pre- } \\
\text { cipitation }\end{array}$ & $\begin{array}{l}\text { - Performance metric } \\
\text { shown (e.g., NSE, } \\
\text { KGE; with colored } \\
\text { levels) } \\
\text { - Blank areas with too } \\
\text { low forecast perfor- } \\
\text { mance (based on } \\
\text { NSE, KGE) }\end{array}$ & $\begin{array}{l}\text { - River discharge; } \\
\text { - Water level; } \\
\text { - Precipitation }\end{array}$ & $\begin{array}{l}\text { - Return periods } \\
\text { (simulation); } \\
\text { - Return periods (ob- } \\
\text { servations; only } \\
\text { stations); } \\
\text { - Selected historic } \\
\text { years (e.g., 2012); } \\
\text { - User defined } \\
\text { thresholds for spe- } \\
\text { cific location }\end{array}$ & $\begin{array}{l}\text { - Excel table for se- } \\
\text { lected station; } \\
\text { - Map of displayed var- } \\
\text { iables (PNG); } \\
\text { - Map of variables } \\
\text { (Shapefile); } \\
\text { - Charts, Graphs (e.g., } \\
\text { histogram, pie, bars) }\end{array}$ & $\begin{array}{l}\text { Interactive } \\
\text { website } \\
\text { (zoom, click, } \\
\text { etc.) }\end{array}$ & $\begin{array}{l}\text { - En; } \\
\text { - Fr; } \\
\text { - Pt; } \\
\text { - Ar }\end{array}$ & $\begin{array}{l}\text { - Automatic alerts to agencies, } \\
\text { that can forward alerts to } \\
\text { stakeholders via existing dis- } \\
\text { tribution channels; } \\
\text { - Automatic alerts to agencies } \\
\text { that can authorize system to } \\
\text { forward alert to stakeholders }\end{array}$ \\
\hline $\begin{array}{l}\text { g_At- } \\
\text { tractve }\end{array}$ & $\begin{array}{l}\text { - Most at- } \\
\text { tractive }\end{array}$ & $\begin{array}{l}\text { - In situ: wa- } \\
\text { ter level, } \\
\text { river dis- } \\
\text { charge; } \\
\text { - EO: water } \\
\text { level, pre- } \\
\text { cipitation }\end{array}$ & $\begin{array}{l}\text { - Performance metric } \\
\text { shown (e.g., NSE, } \\
\text { KGE; with colored } \\
\text { levels) } \\
\text { - Blank areas with too } \\
\text { low forecast perfor- } \\
\text { mance (based on } \\
\text { NSE, KGE) }\end{array}$ & $\begin{array}{l}\text { - River discharge; } \\
\text { - Water level; } \\
\text { - Precipitation; } \\
\text { - Evaporation } \\
\text { - Soil moisture } \\
\text { storage; } \\
\text { - Water quality }\end{array}$ & $\begin{array}{l}\text { - Return periods } \\
\text { (simulation); } \\
\text { - Return periods (ob- } \\
\text { servations; only } \\
\text { stations); } \\
\text { - Selected historic } \\
\text { years (e.g., 2012); } \\
\text { - User defined } \\
\text { thresholds for spe- } \\
\text { cific location }\end{array}$ & $\begin{array}{l}\text { - Excel table for se- } \\
\text { lected station; } \\
\text { - Map of displayed var- } \\
\text { iables (PNG); } \\
\text { - Map of variables } \\
\text { (Shapefile); } \\
\text { - Charts, Graphs (e.g., } \\
\text { histogram, pie, bars) }\end{array}$ & $\begin{array}{l}\text { Interactive } \\
\text { website } \\
\text { (zoom, click, } \\
\text { etc.) }\end{array}$ & $\begin{array}{l}\text { - En; } \\
\text { - } \mathrm{Fr} ; \\
\text { - } \mathrm{Pt} ; \\
\text { - } \mathrm{Ar}\end{array}$ & $\begin{array}{l}\text { - Automatic alerts to agencies, } \\
\text { that can forward alerts to } \\
\text { stakeholders via existing dis- } \\
\text { tribution channels; } \\
\text { - Automatic alerts to agencies } \\
\text { that can authorize system to } \\
\text { forward alert to stakeholders }\end{array}$ \\
\hline h_Equipp & $\begin{array}{l}\text { Fully } \\
\text { equipped }\end{array}$ & $\begin{array}{l}\text { - In situ: wa- } \\
\text { ter level, } \\
\text { river dis- } \\
\text { charge; } \\
\text { - EO: water } \\
\text { level, pre- } \\
\text { cipitation }\end{array}$ & $\begin{array}{l}\text { - Performance metric } \\
\text { shown (e.g., NSE, } \\
\text { KGE; with colored } \\
\text { levels) } \\
\text { - Blank areas with too } \\
\text { low forecast perfor- } \\
\text { mance (based on } \\
\text { NSE, KGE) }\end{array}$ & $\begin{array}{l}\text { - River discharge; } \\
\text { - Water level; } \\
\text { - Precipitation; } \\
\text { - Evaporation } \\
\text { - Soil moisture } \\
\text { storage; } \\
\text { - Water quality }\end{array}$ & $\begin{array}{l}\text { - Return periods } \\
\text { (simulation); } \\
\text { - Return periods (ob- } \\
\text { servations; only } \\
\text { stations); } \\
\text { - Selected historic } \\
\text { years (e.g., 2012); } \\
\text { - User defined } \\
\text { thresholds for spe- } \\
\text { cific location }\end{array}$ & $\begin{array}{l}\text { - Excel table for se- } \\
\text { lected station; } \\
\text { - Map of displayed var- } \\
\text { iables (PNG); } \\
\text { - Map of variables } \\
\text { (Shapefile); } \\
\text { - Charts, Graphs (e.g., } \\
\text { histogram, pie, bars) }\end{array}$ & $\begin{array}{l}\text { - Interactive } \\
\text { website } \\
\text { (zoom, } \\
\text { click, etc.); } \\
\text { - and with } \\
\text { static im- } \\
\text { ages }\end{array}$ & $\begin{array}{l}\text { - En; } \\
\text { - Fr; } \\
\text { - } \mathrm{Pt} ; \\
\text { - Ar }\end{array}$ & $\begin{array}{l}\text { - Automatic alerts to agencies, } \\
\text { that can forward alerts to } \\
\text { stakeholders via existing dis- } \\
\text { tribution channels; } \\
\text { - Automatic alerts to agencies } \\
\text { that can authorize system to } \\
\text { forward alert to stakeholders }\end{array}$ \\
\hline i_Calibr & $\begin{array}{l}\text { Recalibrated } \\
\text { HYPE mod- } \\
\text { els }\end{array}$ & $\begin{array}{l}\text { - In situ: wa- } \\
\text { ter level, } \\
\text { river dis- } \\
\text { charge; }\end{array}$ & $\begin{array}{l}\text { - Performance metric } \\
\text { shown (e.g., NSE, } \\
\text { KGE; with colored } \\
\text { levels) }\end{array}$ & $\begin{array}{l}\text { - River discharge; } \\
\text { - Water level; } \\
\text { - Precipitation; } \\
\text { - Evaporation }\end{array}$ & $\begin{array}{l}\text { Return periods (sim- } \\
\text { ulation) }\end{array}$ & $\begin{array}{l}\text { - Excel table for se- } \\
\text { lected station; } \\
\text { - Map of displayed var- } \\
\text { iables (PNG); }\end{array}$ & $\begin{array}{l}\text { Interactive } \\
\text { website } \\
\text { (zoom, click, } \\
\text { etc.) }\end{array}$ & $\begin{array}{l}\text { - En; } \\
\text { - Fr; } \\
\text { - Pt }\end{array}$ & $\begin{array}{l}\text { - Automatic alerts to agencies, } \\
\text { that can forward alerts to } \\
\text { stakeholders via existing dis- } \\
\text { tribution channels; }\end{array}$ \\
\hline
\end{tabular}




\begin{tabular}{|c|c|c|c|c|c|c|c|c|c|}
\hline Code & $\begin{array}{l}\text { System } \\
\text { Configura- } \\
\text { tion }\end{array}$ & $\begin{array}{l}\text { Observed } \\
\text { variable }\end{array}$ & $\begin{array}{l}\text { Model performance, } \\
\text { accuracy }\end{array}$ & $\begin{array}{l}\text { Forecasted varia- } \\
\text { ble }\end{array}$ & $\begin{array}{l}\text { Flood hazard refer- } \\
\text { ence threshold type }\end{array}$ & Data download & Interactivity & $\begin{array}{l}\text { Lan- } \\
\text { guage }\end{array}$ & $\begin{array}{l}\text { Alert notification to operating } \\
\text { agencies and stakeholders }\end{array}$ \\
\hline & & $\begin{array}{l}\text { - EO: water } \\
\text { level, pre- } \\
\text { cipitation }\end{array}$ & $\begin{array}{l}\text { - Blank areas with too } \\
\text { low forecast perfor- } \\
\text { mance (based on } \\
\text { NSE, KGE) }\end{array}$ & & & $\begin{array}{l}\text { - Map of variables } \\
\text { (Shapefile); } \\
\text { - Charts, Graphs (e.g., } \\
\text { histogram, pie, bars) }\end{array}$ & & & $\begin{array}{l}\text { - Automatic alerts to agencies } \\
\text { that can authorize system to } \\
\text { forward alert to stakeholders }\end{array}$ \\
\hline j_Cal-EO & $\begin{array}{l}\text { Recalibrated } \\
\text { HYPE mod- } \\
\text { els and EO } \\
\text { data }\end{array}$ & $\begin{array}{l}\text { - In situ: wa- } \\
\text { ter level, } \\
\text { river dis- } \\
\text { charge; } \\
\text { - EO: water } \\
\text { level, pre- } \\
\text { cipitation }\end{array}$ & $\begin{array}{l}\text { - Performance metric } \\
\text { shown (e.g., NSE, } \\
\text { KGE; with colored } \\
\text { levels) } \\
\text { - Blank areas with too } \\
\text { low forecast perfor- } \\
\text { mance (based on } \\
\text { NSE, KGE) }\end{array}$ & $\begin{array}{l}\text { - River discharge; } \\
\text { - Water level; } \\
\text { - Precipitation; } \\
\text { - Evaporation }\end{array}$ & $\begin{array}{l}\text { Return periods (simu- } \\
\text { lation) }\end{array}$ & $\begin{array}{l}\text { - Excel table for se- } \\
\text { lected station; } \\
\text { - Map of displayed var- } \\
\text { iables (PNG); } \\
\text { - Map of variables } \\
\text { (Shapefile); } \\
\text { - Charts, Graphs (e.g., } \\
\text { histogram, pie, bars) }\end{array}$ & $\begin{array}{l}\text { Interactive } \\
\text { website } \\
\text { (zoom, click, } \\
\text { etc.) }\end{array}$ & $\begin{array}{l}\text { - En; } \\
\text { - Fr; } \\
\text { - Pt }\end{array}$ & $\begin{array}{l}\text { - Automatic alerts to agencies, } \\
\text { that can forward alerts to } \\
\text { stakeholders via existing dis- } \\
\text { tribution channels; } \\
\text { - Automatic alerts to agencies } \\
\text { that can authorize system to } \\
\text { forward alert to stakeholders }\end{array}$ \\
\hline $\begin{array}{l}\text { k_Cal- } \\
\text { EO-situ }\end{array}$ & $\begin{array}{l}\text { Recalibrated } \\
\text { HYPE mod- } \\
\text { els and EO } \\
\text { data }\end{array}$ & $\begin{array}{l}\text { - In situ: wa- } \\
\text { ter level, } \\
\text { river dis- } \\
\text { charge; } \\
\text { - EO: water } \\
\text { level, pre- } \\
\text { cipitation }\end{array}$ & $\begin{array}{l}\text { - Performance metric } \\
\text { shown (e.g., NSE, } \\
\text { KGE; with colored } \\
\text { levels) } \\
\text { - Blank areas with too } \\
\text { low forecast perfor- } \\
\text { mance (based on } \\
\text { NSE, KGE) }\end{array}$ & $\begin{array}{l}\text { - River discharge; } \\
\text { - Water level; } \\
\text { - Precipitation; } \\
\text { - Evaporation }\end{array}$ & $\begin{array}{l}\text { Return periods (simu- } \\
\text { lation) }\end{array}$ & $\begin{array}{l}\text { - Excel table for se- } \\
\text { lected station; } \\
\text { - Map of displayed var- } \\
\text { iables (PNG); } \\
\text { - Map of variables } \\
\text { (Shapefile); } \\
\text { - Charts, Graphs (e.g., } \\
\text { histogram, pie, bars) }\end{array}$ & $\begin{array}{l}\text { Interactive } \\
\text { website } \\
\text { (zoom, click, } \\
\text { etc.) }\end{array}$ & $\begin{array}{l}\text { - En; } \\
\text { - Fr; } \\
\text { - Pt }\end{array}$ & $\begin{array}{l}\text { - Automatic alerts to agencies, } \\
\text { that can forward alerts to } \\
\text { stakeholders via existing dis- } \\
\text { tribution channels; } \\
\text { - Automatic alerts to agencies } \\
\text { that can authorize system to } \\
\text { forward alert to stakeholders }\end{array}$ \\
\hline
\end{tabular}




\subsection{Predicting performance of each system configuration}

\subsubsection{Attribute details}

\subsection{High accuracy of information}

Description: Hydrological forecasts and flood risk alerts should consistently reach high accuracy (matching hydrological observations and local knowledge of flood occurrence and magnitude).

Attribute: Value of KGE index for 1, 3, 10 day forecasts (11_kge)

Unit: $\quad$ value [0:1]

Description: Level of accuracy consisting of $K G E$ for 1, 3, and 10 day forecasts index transformed into [0:1] value by aggregation with a weighted sum using different weights for the sub-attributes.

Best case: Operational forecasts are always 100\% accurate and have no errors $(K G E=1)$ across the 1, 3, and 10 lead days.

Worst case: Forecasts are 0\% accurate and misleading (KGE $\left.=-\infty /-1^{\prime} 000\right)$ across the 1, 3, and 10 lead days.

Addit. Info: The accuracy refers to the streamflow (fluvial floods) information sent out (disseminated forecasts and alerts). The manual operator may decide to send it or not.

\section{Attribute details:}

11_kge is an artificial (constructed) attribute, consisting of the three sub-attributes (KGE index for 1 day, 3 day, and 10 day forecasts).

There are several indices used for accuracy in hydrology (see e.g., Deliverable 3.2, section 5 in https://fanfar.eu/wp-content/uploads/sites/4/2020/05/FANFAR-D3.2-Hydrological-Models.pdf). The FANFAR attribute accuracy however is solely measured through the KGE index (Kling-Gupta Efficiency; Gupta et al., 2009), which is commonly used in hydrology for model evaluation; i.e., to estimate the error of a set predicted values vs observed values. To predict the performance of the FANFAR forecasting system towards the observed streamflow, we used expert estimates. These were elicited from Jafet Andersson (SMHI, hydrologist from FANFAR) in July 2019. He estimated the KGE index over three typically used lead days: the 1 day, 3 day, and 10 day forecasts. These lead days indicate the time between the forecast production and the actual flood event. We used 7 levels for the assessment, which initially had a (continuous linear) level ranging from 0 (worst), over very bad (16.667), (...), to very good (83.333), and 100 (best). We later transformed the levels to a value from 0 to 1 . The expert assigned a KGE index number to each level for each of the three lead days (

Table SI-8). Thereafter, each configuration (FANFAR system configuration) received a KGE index number for each of the three lead days (

Table SI-9). This KGE index number was transformed to a value using a nonlinear value function between all the above levels from 0 (worst) to 1 (best; with linear interpolation between the levels from worst to best;

Table SI-8; Figure SI-5). We then aggregated all three lead day values into a single value (between 0 and 1) with a weighted sum, where the lead times $(1,3$, and 10 days) received different weights. The accuracy of the 1 day forecast was weighted the highest $[\mathrm{w}=0.5]$, the 3 day forecast received a slightly lower weight $[\mathrm{w}=0.4]$, and the 10 day forecast was given small importance $[\mathrm{w}=0.1]$. As consequence of the transformation of the sub-attributes into one [0:1] value, a linear value function $[0: 1]$ is used for this attribute for the subsequent MCDA modelling.

\section{Uncertainty of predictions:}

We dealt with the uncertainty of each single lead-day estimate using triangle distributions. These contain a best (most probable) expert estimate for the 1, 3, and 10 day estimate, and a lower (minimum) / upper bound (maximum) of uncertainty in the expert's statement. The uncertainty of the sub-attributes is not symmetrical around the prediction. The uncertainty distributions of the sub-attributes are skewed towards lower values, as for many reasons, the lower values in the uncertainty range are more likely, than higher values. To aggregate over the three sub-attributes, we used 1'000 Monte Carlo simulation runs, each of 
them drawing from the triangle uncertainty distribution of each sub-attribute. For the subsequent MCDA, we used a normal distribution to model the aggregated uncertainty of the three sub-attributes. To estimate the shape of the aggregated uncertainty distribution, we chose a normal distribution with mean $=$ mean of Monte Carlo simulation, and standard deviation $=1 / 4$ of the $95 \%$ confidence interval resulting from the Monte Carlo simulation with 1'000 runs. This resulted in an individual uncertainty distribution for each configuration for this attribute. These distributions are symmetrical but have a lower mean than the aggregated prediction to account for the skewedness of uncertainty (Figure SI-6).

Table SI-8. Expert estimates for levels of sub-attributes: KGE-index for 1, 3, and 10 day forecasts (lead days).

\begin{tabular}{lr|rrr}
\hline $\begin{array}{l}\text { Error of forecast } \\
\text { level }\end{array}$ & Value & \multicolumn{3}{|c}{ KGE (lead days) } \\
\hline Best & 1.000 & 1.00 & 1.00 & KGE-10 \\
Very good & 0.833 & 0.90 & 0.80 & 1.00 \\
Good & 0.667 & 0.70 & 0.60 & 0.50 \\
Neutral & 0.500 & 0.40 & 0.30 & 0.25 \\
Bad & 0.333 & 0.00 & -0.50 & 0.00 \\
Very bad & 0.167 & -1.00 & -2.00 & -1.00 \\
Worst & 0.000 & $\infty \mid-1^{\prime} 000.00$ & $\infty \mid-1^{\prime} 000.00$ & $\infty \mid-1^{\prime} 000.00$ \\
\hline \hline
\end{tabular}

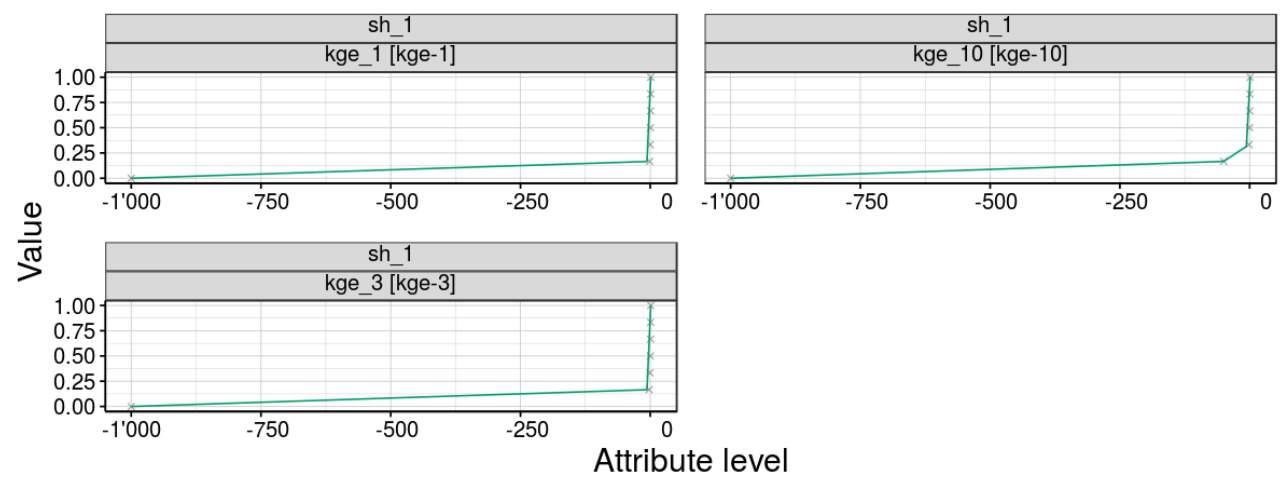

Figure SI-5: Value functions to aggregate the three sub-attributes kge-1, kge-3, and kge-10 into one [0:1] value. The functions were approximated from the level estimates by the expert using linear interpolation with 140 approximated points.

Table SI-9: Predictions of the three sub-attributes kge-1, kge-3, and kge-10 for each system configuration with min/ max values of uncertainty in the estimate. These predictions are expert estimates done by Jafet Andersson and were aggregated into one value (last column), which is the value used as prediction for the aggregated attribute "11_kge" in the subsequent MCDA modelling.

\begin{tabular}{|c|c|c|c|c|c|c|c|c|c|c|}
\hline $\begin{array}{l}\text { System con- } \\
\text { figurations }\end{array}$ & $\begin{array}{r}\text { Pre- } \\
\text { dicted } \\
\text { KGE } \\
\text { (1 day) }\end{array}$ & $\begin{array}{r}\min \\
\text { (1 day) }\end{array}$ & $\begin{array}{r}\max \\
\text { (1 day) }\end{array}$ & $\begin{array}{r}\text { Pre- } \\
\text { dicted } \\
\text { KGE } \\
(3 \text { days })\end{array}$ & $\begin{array}{r}\min \\
\text { (3 days) }\end{array}$ & $\begin{array}{r}\max \\
\text { (3 days) }\end{array}$ & $\begin{array}{r}\text { Pre- } \\
\text { dicted } \\
\text { KGE } \\
(10 \\
\text { days) }\end{array}$ & $\begin{array}{r}\min \\
(10 \\
\text { days })\end{array}$ & $\begin{array}{r}\max \\
(10 \\
\text { days })\end{array}$ & $\begin{array}{l}\text { Aggre- } \\
\text { gated } \\
\text { value of } \\
\text { predic- } \\
\text { tion }\end{array}$ \\
\hline a_Fast-dev & 0.30 & 0.00 & 0.60 & 0.15 & -0.45 & 0.35 & -2.00 & -20.00 & 0.20 & 0.450 \\
\hline b_Res-user & 0.79 & 0.00 & 0.88 & 0.49 & 0.00 & 0.61 & -1.94 & -19.58 & 0.22 & 0.646 \\
\hline c_Easy-use & 0.42 & 0.00 & 0.67 & 0.24 & -0.31 & 0.42 & -1.99 & -19.90 & 0.20 & 0.484 \\
\hline d_Fast & 0.46 & 0.00 & 0.69 & 0.26 & -0.26 & 0.43 & -1.98 & -19.87 & 0.20 & 0.497 \\
\hline e_Consent & 0.86 & 0.00 & 0.92 & 0.58 & 0.00 & 0.68 & 0.40 & -3.20 & 0.84 & 0.738 \\
\hline f_Robust & 0.46 & 0.00 & 0.69 & 0.26 & -0.26 & 0.43 & 0.40 & -3.20 & 0.84 & 0.540 \\
\hline g_Attractve & 0.86 & 0.00 & 0.92 & 0.58 & 0.00 & 0.68 & 0.40 & -3.20 & 0.84 & 0.738 \\
\hline h_Equipp & 0.86 & 0.00 & 0.92 & 0.58 & 0.00 & 0.68 & 0.40 & -3.20 & 0.84 & 0.738 \\
\hline i_Calibr & 0.65 & 0.00 & 0.80 & 0.49 & 0.00 & 0.61 & 0.40 & -3.20 & 0.84 & 0.638 \\
\hline j_Cal-EO & 0.72 & 0.00 & 0.84 & 0.58 & 0.00 & 0.68 & 0.40 & -3.20 & 0.84 & 0.679 \\
\hline k_Cal-EO-situ & 0.93 & 0.00 & 0.96 & 0.75 & 0.00 & 0.81 & 0.40 & -3.20 & 0.84 & 0.833 \\
\hline
\end{tabular}



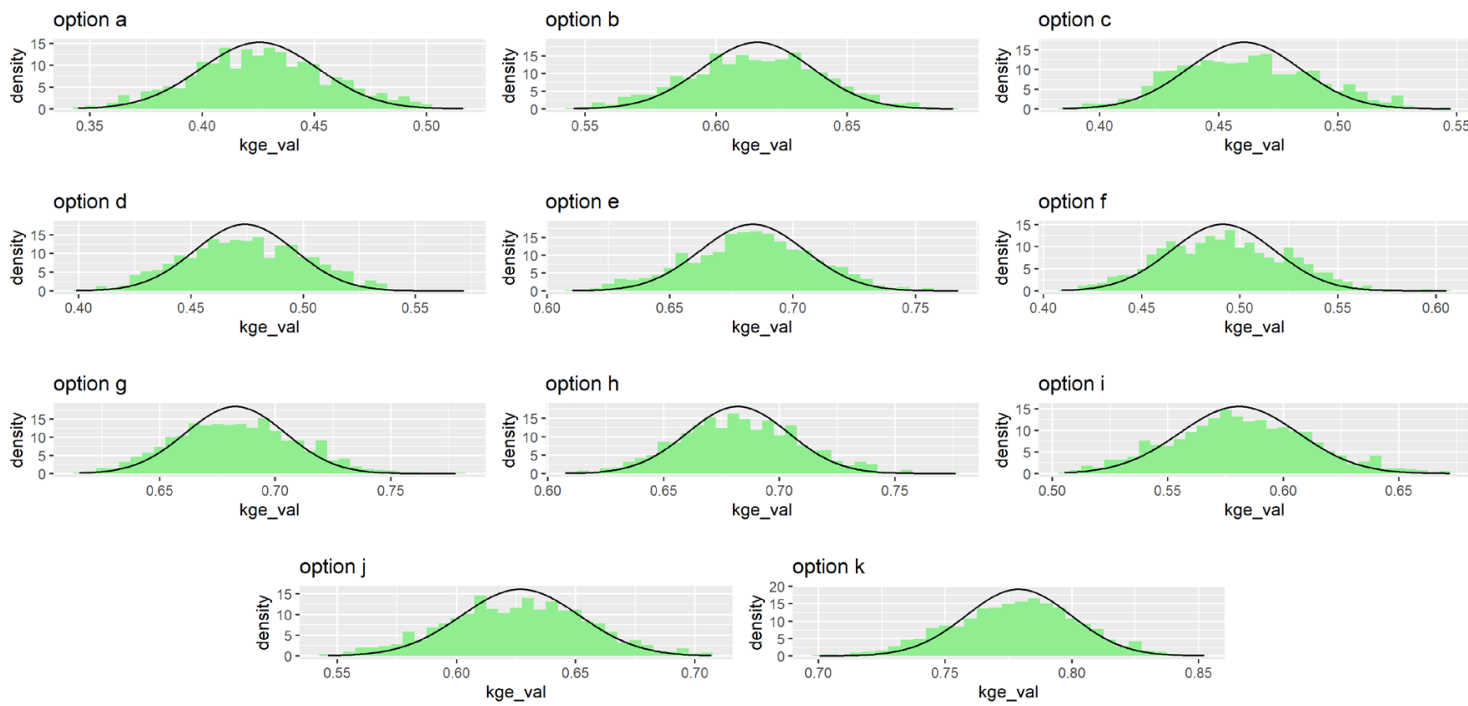

Figure SI-6: Distribution of resulting uncertainties (green) after aggregating the three sub-attributes and 1'000 Monte Carlo simulation runs. Results for the 11 FANFAR system configurations $\mathbf{a}-\mathbf{k}$. A normal distribution with mean = mean of Monte Carlo simulation, and sd $=1 / 4$ of the $95 \%$ confidence interval (black curve) fits the resulting uncertainty well. A normal distribution is thus used as input of the uncertainty of the aggregated prediction of the attribute 11 kge in the subsequent MCDA. The means of these distributions are lower than the aggregated predictions in

Table SI-9 to account for skewedness of the uncertainty in the expert estimates.
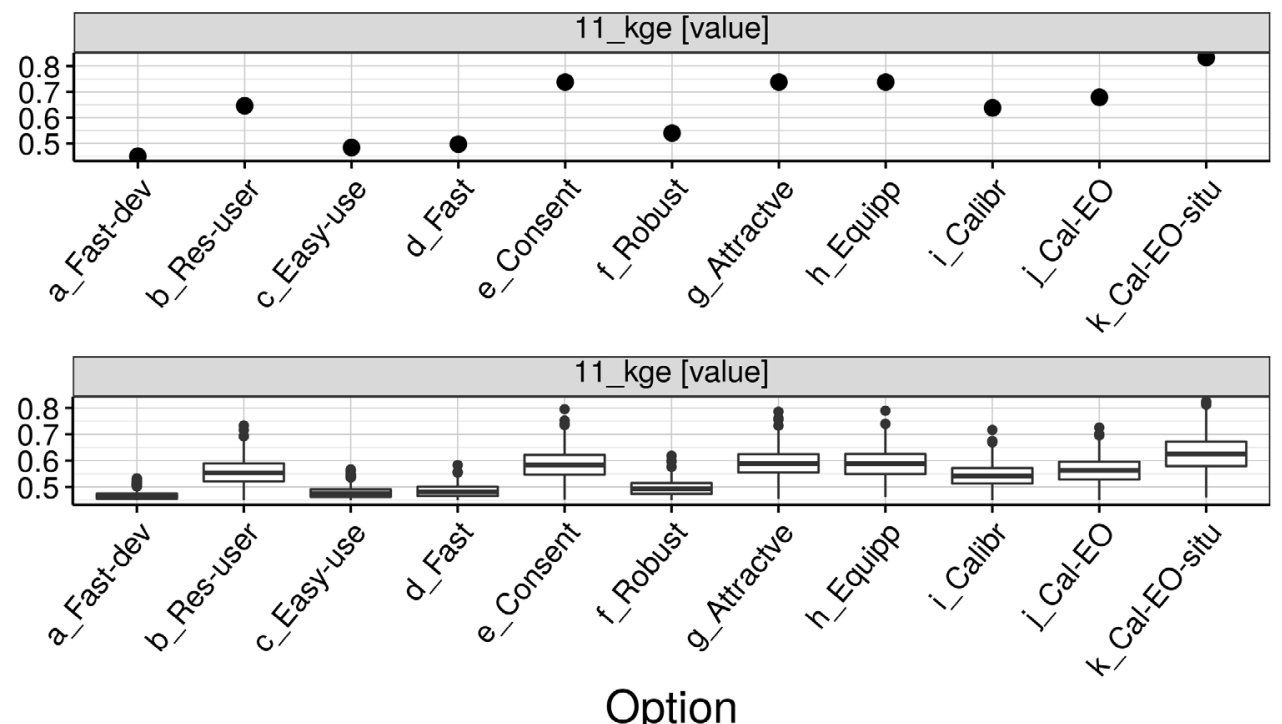

Figure SI-7: Visualization of the aggregated value of predictions of all system configurations for the attribute $11 \mathrm{kge}$. The plot on top represents the predictions without uncertainty, while the plot below shows the uncertainty distribution resulting from the Monte Carlo Simulation with 1000 runs.

\subsection{Clear flood risk information}

Description: Flood risk thresholds should be clearly defined to be used to calculate flood risks, and are easy to understand and apply in flood management

Attribute: Clarity of flood risk thresholds in risk management (12_info)

Unit: $\quad$ value [0:1]

Description: Levels of flood risk threshold of three categorical sub-attributes: 1) location, 2) possibility to calculate risk, 3) ease of understanding in applied risk management aggregated into [0:1] value, using an equally weighted sum. 
Best case: Flood risk thresholds are clearly defined everywhere. No ambiguities of calculating flood risk or understanding and application.

Worst case: No flood risk definitions. No adequate notifications can be derived, distributed, understood or applied.

\section{Attribute details:}

12_info is an artificial (constructed) attribute, consisting of the three categorical sub-attributes location, possibility of flood risk calculation, and ease of application to flood management.

Our definition of the flood risk thresholds depends on: 1) place where they are defined (e.g., everywhere or gauged locations); 2) possibility to calculate the flood risk; and 3) how easy the flood risk thresholds are to apply in flood management. Estimates on these categorical sub-attributes were elicited from Bernard Minoungou (AGRHYMET, hydrologist from FANFAR) in July 2019. We used 7 levels for the assessment, which initially had a (continuous linear) level ranging from 0 (worst), over very bad (16.667), (...), to very good (83.333), and 100 (best). We later transformed the levels to a value from 0 to 1 . To each level, the expert assigned a combination of the three categorical sub-attributes (

Table SI-10). Note, when giving the predictions for each of our FANFAR system configurations, the expert later decided that our configurations are all defined "everywhere", and that it is possible to calculate the flood risk for all configurations. For these two sub-attributes a value of 1 is achieved by all configurations in our case, and thus they are not needed to evaluate the currently chosen FANFAR system configurations. Therefore, we only used yes (1) / no (0) categorical input data for the subattribute on ease of application to flood management. Consequently, the respective values of the configurations are either 1 (best-possible level), or 0.667 (good level;

Table SI-10 bold text). As consequence of the transformation of the sub-attributes into one [0:1] value, a linear value function $[0: 1]$ is used for this attribute for the subsequent MCDA (Figure SI-8).

\section{Uncertainty of predictions:}

For this attribute there was no uncertainty in the experts' estimate.

Table SI-10: Expert estimates for levels of the flood risk threshold for the three categorical sub-attributes 1) location, 2) possibility of flood risk calculation, and 3) ease of application to flood management. In our case we only considered sub-attribute 3, as all configurations are identical for sub-attribute 1 , and 2.

\begin{tabular}{ll|lll}
\hline \hline $\begin{array}{l}\text { Flood risk threshold } \\
\text { definition level }\end{array}$ & Value & $\begin{array}{l}\text { Place where flood risk } \\
\text { is defined }\end{array}$ & $\begin{array}{l}\text { Possible to calculate } \\
\text { flood risk? }\end{array}$ & $\begin{array}{l}\text { Possible to apply to } \\
\text { flood management? }\end{array}$ \\
\hline Best & 1.000 & Everywhere & Yes & Yes \\
Very good & 0.833 & Gauged loc. & Yes & Yes \\
Good & 0.667 & Everywhere & Yes & No \\
Neutral & 0.500 & Gauged loc. & Yes & No \\
Bad & 0.333 & Everywhere & No & Yes \\
Bad & 0.333 & Gauged loc. & No & Yes \\
Very bad & 0.167 & Everywhere & No & No \\
Very bad & 0.167 & Gauged loc. & No & No \\
Worst & 0.000 & Not defined & No & No \\
\hline \hline
\end{tabular}

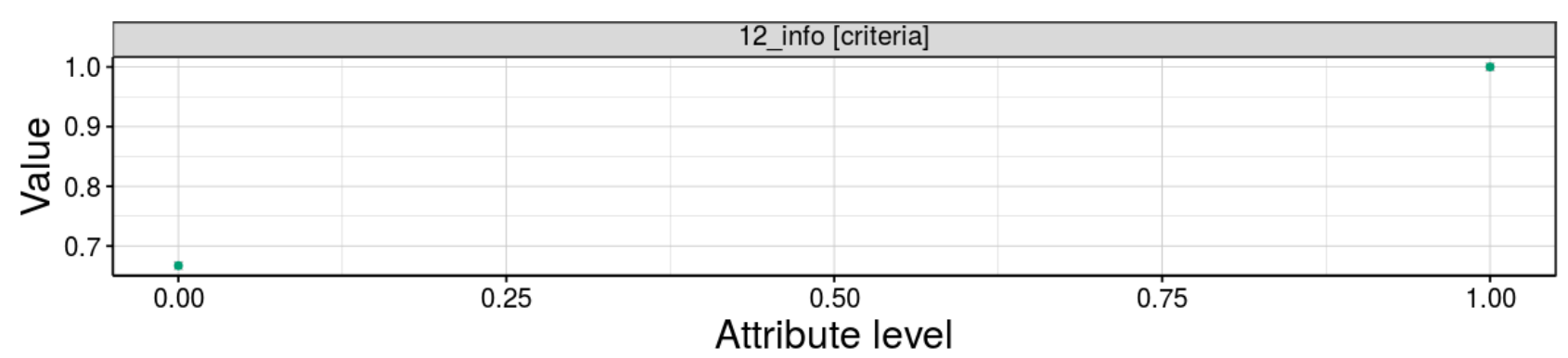

Figure SI-8: Discrete value function for the transformation of the categorical sub-attribute on ease of application to flood management into a [1:0] value. A sub-attribute category "No" $(0)$ achieved the level "Good" (value = 0.667$)$, and a category "Yes" (1) resulted in the 
best case (value = 1). If there would have been differences between configurations concerning the levels of the other two sub-attributes, a more complex aggregation approach would be required.

Table SI-11: Predictions on the three categorical sub-attributes of 12 info for each configuration. All configurations are defined everywhere and enable to calculate flood risk, thus only the ease of understanding in applied risk management is important for the FANFAR system configurations. Consequently, the aggregated value for the subsequent MCDA is either 0.667 or 1.

\begin{tabular}{llllr}
\hline $\begin{array}{l}\text { System configura- } \\
\text { tions }\end{array}$ & Prediction (place) & $\begin{array}{l}\text { Prediction (calcu- } \\
\text { lation risk) }\end{array}$ & $\begin{array}{l}\text { Prediction (understand- } \\
\text { able for flood manage- } \\
\text { ment) }\end{array}$ & $\begin{array}{r}\text { Aggregated value } \\
\text { of prediction }\end{array}$ \\
\hline a_Fast-dev & Everywhere & Yes & No & 0.667 \\
b_Res-user & Everywhere & Yes & Yes & 1.000 \\
c_Easy-use & Everywhere & Yes & Yes & 1.000 \\
d_Fast & Everywhere & Yes & No & 0.667 \\
e_Consent & Everywhere & Yes & Yes & 1.000 \\
f_Robust & Everywhere & Yes & Yes & 1.000 \\
g_Attractve & Everywhere & Yes & Yes & 1.000 \\
h_Equipp & Everywhere & Yes & Yes & 1.000 \\
i_Calibr & Everywhere & Yes & No & 0.667 \\
j_Cal-EO & Everywhere & Yes & No & 0.667 \\
k_Cal-EO-situ & Everywhere & Yes & No & 0.667 \\
\hline
\end{tabular}

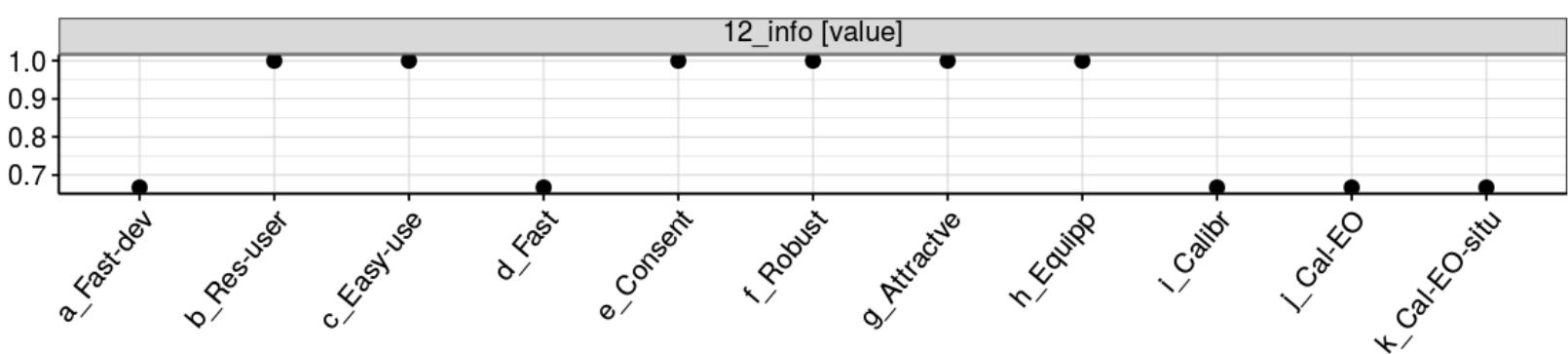

Figure SI-9: Visualization of the aggregated value of predictions of all system configurations for the attribute 12 info. There was no uncertainty defined for this attribute.

\subsection{Reliable access to information}

Description: Access to forecasts and flood risk notifications should be reliable: always reach intended recipients / key stakeholders.

Attribute: Stability of distribution channel (21_channel)

Unit: $\quad$ score [0:2.2]

Description: Access to information calculated by the weighted sum of categorical stability of access (i.e., stable, sporadic, none) for each distribution channel (i.e., SMS, Email, fanfar.eu).

Best case: Forecasts and flood risk notifications always reach the intended stakeholders $[\Sigma=2.2]$.

Worst case: Forecasts and flood risk notifications do not reach the intended stakeholders [ $\Sigma=0]$.

\section{Attribute details:}

21_channel is an artificial (constructed) attribute, consisting of the weighted sum of the reliability of the most important distribution channels: SMS, Email, and FANFAR homepage.

For each configuration (FANFAR system configuration) and each distribution channel, i.e., SMS, Email, FANFAR homepage (fanfar.eu), the reliability of access was assigned categorically as a value, i.e., stable [v=1], sporadic [v=0.7], none [v=0]. This estimate was done by Francisco Silva Pinto (Eawag) in June 2019 (Table SI-13). To obtain a single overall score for each configuration, we calculated the weighted sum over all three distribution channels, with following weights: SMS [w=1], Email $[\mathrm{w}=0.7]$, homepage $[\mathrm{w}=0.5]$. Thereafter, we used 7 assessment levels, which initially had a (continuous linear) level ranging 
from 0 (worst), over very bad (16.667), (...), to very good (83.333), and 100 (best; Table SI-12Table SI-13). After the transformation into values from 0 to 1 , these levels serve as a nonlinear marginal value function based on the expert estimate for the subsequent MCDA (Figure SI-10).

\section{Uncertainty of predictions:}

According to Francisco Silva Pinto, who did the predictions, the estimates have an uncertainty of a normal distribution with $10 \%$ of the prediction as standard deviation.

Table SI-12: Expert estimates for levels of the weighted sum (score) of the reliability of access to the three distribution channels, SMS, Email, and homepage (fanfar.eu). Note: These levels are directly used for the marginal value function in the subsequent MCDA modelling.

\begin{tabular}{lr|r}
\hline \hline $\begin{array}{l}\text { Reliability of distribution } \\
\text { channel level }\end{array}$ & Value & Weighted sum (score) \\
\hline Best & 1.000 & 2.20 \\
Very good & 0.833 & 2.05 \\
Good & 0.667 & 1.84 \\
Neutral & 0.500 & 1.54 \\
Bad & 0.333 & 1.19 \\
Very bad & 0.167 & 0.70 \\
Worst & 0.000 & 0.00 \\
\hline \hline
\end{tabular}

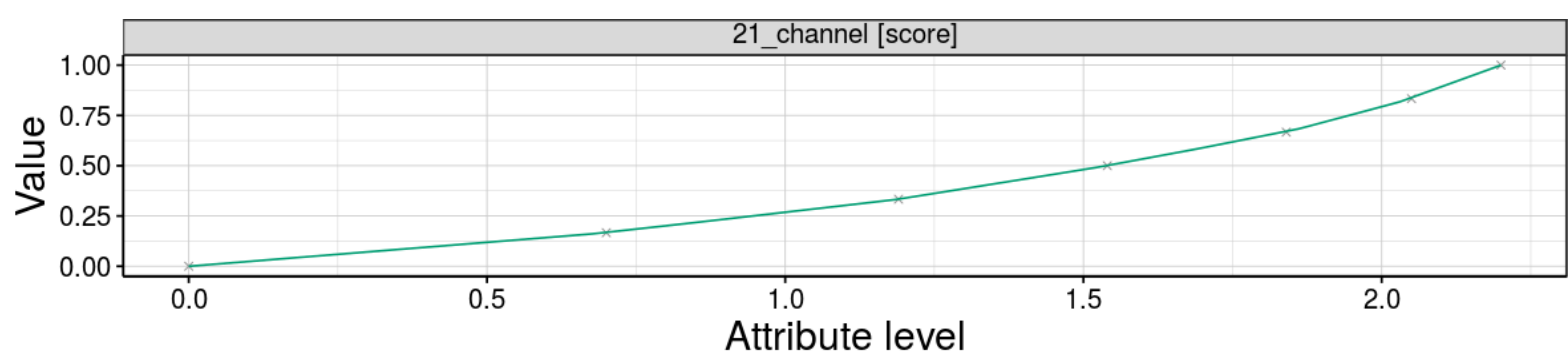

Figure SI-10: Nonlinear marginal value function for the subsequent MCDA modelling of attribute "reliable access to information". The shape of the value function is based on the experts' estimates on levels ( Table SI-12).

Note: In contrast to the previous attributes, the levels here were not used for aggregation of sub-attributes, but as a nonlinear single attribute value function for the subsequent MCDA. In other words, here the predictions were directly adopted for the input of the subsequent MCDA.

Table SI-13: Predictions on the reliability of the three distribution channels SMS, Email, and FANFAR homepage. The predictions were transformed into one score for the subsequent MCDA using a weighted sum with different weights for the distribution channels.

\begin{tabular}{llllr}
\hline System configurations & $\begin{array}{l}\text { Predicted reliabil- } \\
\text { ity of SMS }\end{array}$ & $\begin{array}{l}\text { Predicted reliabil- } \\
\text { ity of Email }\end{array}$ & $\begin{array}{l}\text { Predicted reliability } \\
\text { of fanfar_eu }\end{array}$ & $\begin{array}{r}\text { Total weighted } \\
\text { sum (score) }\end{array}$ \\
\hline a_Fast-dev & none & none & sporadic & 0.35 \\
b_Res-user & stable & stable & sporadic \\
C_Easy-use & sporadic & sporadic & stable & 2.05 \\
d_Fast & stable & stable & stable & 1.69 \\
e_Consent & stable & stable & sporadic & 2.20 \\
f_Robust & stable & stable & sporadic & 2.05 \\
g_Attractve & stable & stable & sporadic & 2.05 \\
h_Equipp & stable & stable & sporadic & 2.05 \\
i_Calibr & stable & stable & sporadic & 2.05 \\
j_Cal-EO & stable & stable & sporadic & 2.05 \\
k_Cal-EO-situ & stable & stable & sporadic & 2.05 \\
\hline
\end{tabular}



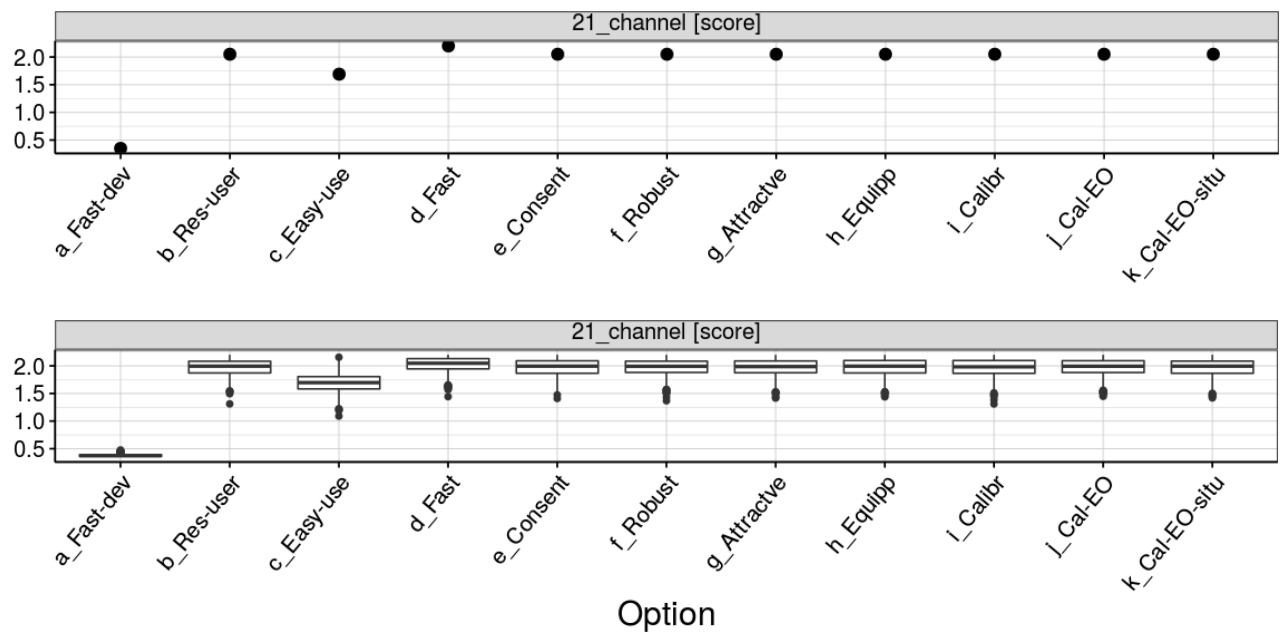

Figure SI-11: Visualization of the total weighted sum of all system configurations used as predictions for the attribute 21_channel. The plot on top represents the predictions without uncertainty, while the plot below shows the uncertainty distribution resulting from the Monte Carlo Simulation with 1'000 runs.

\subsection{Timely production, distribution, and access to information}

Description: The time to produce, distribute and access forecasts and flood risk notifications. A faster system allows for a more timely response.

Attribute: $\quad$ Time to produce, distribute, and access information (22_time)

Unit: $\quad$ value [0:1]

Description: Level of time required to produce forecast, access distribution channels, and access IVP; transformed to [0:1] value with linear interpolation, aggregated with equally weighted sum.

Best case: Flood forecasts and hazard notifications are produced in $0.5 \mathrm{~h}$, and to access the information in West Africa it takes less than 30 seconds on all platforms.

Worst case: Flood forecasts and hazard notifications are produced in more than $24 \mathrm{~h}$. In West Africa, it takes more than 10 min to access the information through the IVP, SMS, and Email, and more than 15 min to access through the production system.

Additional information:

Production time: whenever the first component starts until the last component finishes (Hydrology-TEP) in an optimized production chain. From when there is data on the SMHI FTP until the forecast is finished and sent out.

Access time through distribution channels: time that it takes for a user from when it was sent until it is possible to be seen in West Africa (as in the FANFAR workshops).

Access time through production system: loading time of the Hydrology-TEP and navigation time (imagine someone navigating in West Africa throughout the H-TEP).

\section{Attribute details:}

22 time is an artificial (constructed) attribute consisting of three sub-attributes, the time it takes to: 1) produce a forecast; 2) access the information through the distribution channels; and 3) access the information in the forecast production system.

The predictions for these sub-attributes were estimated by Emmanuel Mathot (Terradue) in July 2019. For the production time, he estimated a basic production time (i.e., the time a system with the least resources (configuration a) requires). To that basic production time, additional time was added depending on whether the system configuration utilizes satellite data $(+0.5 \mathrm{~h})$, local observations $(+0.75 \mathrm{~h})$, or manual control $(+1 \mathrm{~h})$. Criteria for the estimated time needed for access through the distribution channels, were whether the configuration uses SMS, or Email (which both reduce the access time). The access time through the production system depends on the complexity of the components used in each configuration e.g., diversity in observational data inputs, number of models used, type of information derived, and language. The more complex the configuration the more time is needed to load menus and output (Table SI-14). 
Note: During the operational period of the FANFAR pre-operational system in 2020, the production times were generally much higher than what was estimated by the expert in July 2019. However, the assumption, that a more complex system configuration requires more time, still holds. Therefore, the relative differences of the system configurations are still valid.

For each sub-attribute, levels were created, which initially had a (continuous linear) level ranging from 0 (worst), over very bad (16.667), (...), to very good (83.333), and 100 (best; Table SI-14). After the transformation into values from 0 to 1 we used these levels as value functions for the aggregation of the three sub-attributes into one single [0:1] value using a weighted sum with different weights for the sub-attributes. As the production time and access via the distribution channels is more important for the users to access every day, these two sub-attributes received a slightly higher weights (each w=0.4), and access via the production system received a lower weight of 0.2. (Figure SI-12). This resulting [0:1] value of the aggregation forms the prediction for the subsequent MCDA. As consequence of the transformation of the sub-attributes into one [0:1] value, a linear value function $[0: 1]$ is used for this attribute for the subsequent MCDA modelling.

\section{Uncertainty of predictions:}

We dealt with the uncertainty of each sub-attribute estimate using a triangle distribution. This contains a best (most probable) expert estimate for the three time estimates, and a lower (minimum) / upper bound (maximum) for each, which are both very unlikely. The expert initially stated uncertainty ranges exceeding the value function range. These uncertainty ranges were trimmed off to lie within the value function, as any predictions outside the value function range will achieve a value of 0 (if undershooting) or 1 (if overshooting). To aggregate over the three sub-attributes, we used 1'000 Monte Carlo simulation runs, each of them drawing from the triangle uncertainty distribution of each sub-attribute. For the subsequent MCDA, we used a normal distribution to model the aggregated uncertainty of the three sub-attributes. To estimate the shape of the aggregated uncertainty distribution, we chose to utilize a normal distribution with mean = mean of Monte Carlo simulation, and standard deviation $=1 / 4$ of the $95 \%$ confidence interval resulting from the Monte Carlo simulation with 1'000 runs. This resulted in an individual uncertainty distribution for each configuration for this attribute (Figure SI-13).

Table SI-14: Expert estimates for levels of the three sub-attributes time to 1) produce, 2) access via distribution channels, and 3) access the flood risk information in the forecast production system.

\begin{tabular}{lr|rrr}
\hline \hline $\begin{array}{l}\text { Timely information } \\
\text { level }\end{array}$ & Value & Production time $(\mathrm{h})$ & $\begin{array}{r}\text { Access via distribution } \\
\text { channel (min) }\end{array}$ & $\begin{array}{r}\text { Access via produc- } \\
\text { tion system (min) }\end{array}$ \\
\hline Best & 1.000 & 0.5 & 0.5 & 0.5 \\
Very good & 0.833 & 1.0 & 1.0 & 2.0 \\
Good & 0.667 & 2.0 & 2.0 & 5.0 \\
Neutral & 0.500 & 3.0 & 3.5 & 7.5 \\
Bad & 0.333 & 6.0 & 5.0 & 10.0 \\
Very bad & 0.167 & 12.0 & 7.5 & 12.5 \\
Worst & 0.000 & 24.0 & 10.0 & 15.0 \\
\hline \hline
\end{tabular}

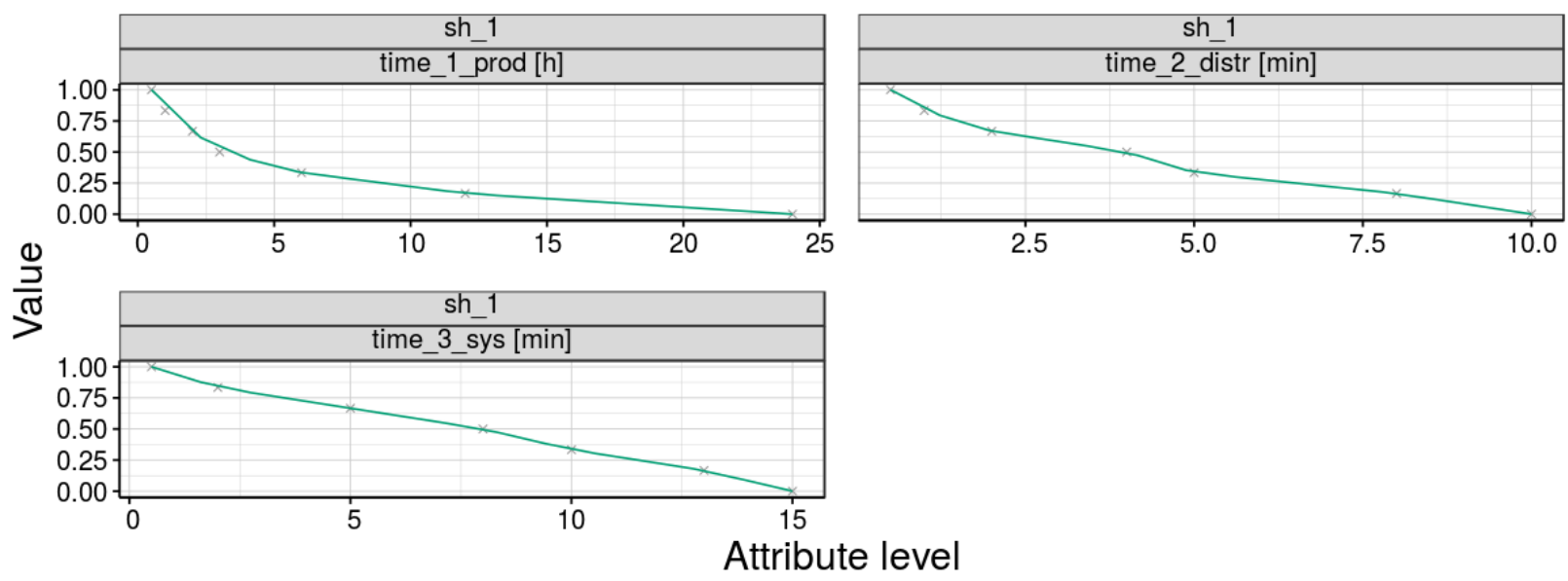

Figure SI-12: Value functions used for the aggregation of the three sub-attributes into one [0:1] value. The functions were approximated from the level estimates by the expert using linear interpolation with 14 approximated points. 
Table SI-15: Predictions of the three sub-attributes production time, access time through distribution channel, and access time through production system for each system configuration with $\mathrm{min} / \mathrm{max}$ values of uncertainty in the estimate. These predictions are expert estimates done by Emmanuel Mathot and were aggregated into one value (last column), which is the value used as prediction for the aggregated attribute 22_time in the subsequent MCDA modelling.

\begin{tabular}{|c|c|c|c|c|c|c|c|c|c|c|}
\hline $\begin{array}{l}\text { System configu- } \\
\text { rations }\end{array}$ & $\begin{array}{l}\text { Predicted } \\
\text { production } \\
\text { time }(\mathrm{h})\end{array}$ & Min & Max & $\begin{array}{l}\text { Predicted } \\
\text { access time } \\
\text { distr ch } \\
\text { (min) }\end{array}$ & Min & Max & $\begin{array}{l}\text { Predicted } \\
\text { access time } \\
\text { prod syst } \\
\text { (min) }\end{array}$ & Min & Max & $\begin{array}{l}\text { Aggregated } \\
\text { value of } \\
\text { prediction }\end{array}$ \\
\hline a_Fast-dev & 4.0 & 2.0 & 6.0 & 2.0 & 1.0 & 4.0 & 10.0 & 5.0 & 15.0 & 0.511 \\
\hline b_Res-user & 4.0 & 2.0 & 6.0 & 0.5 & 0.3 & 1.0 & 12.0 & 6.0 & 15.0 & 0.618 \\
\hline c_Easy-use & 5.8 & 3.8 & 7.8 & 1.0 & 0.5 & 2.0 & 11.0 & 5.5 & 15.0 & 0.526 \\
\hline d_Fast & 4.5 & 2.5 & 6.5 & 0.5 & 0.3 & 1.0 & 10.0 & 5.0 & 15.0 & 0.633 \\
\hline e_Consent & 4.5 & 2.5 & 6.5 & 0.5 & 0.3 & 1.0 & 15.0 & 7.5 & 15.0 & 0.567 \\
\hline f_Robust & 6.3 & 4.3 & 8.3 & 0.5 & 0.3 & 1.0 & 13.0 & 6.5 & 15.0 & 0.557 \\
\hline g_Attractve & 5.5 & 3.5 & 7.5 & 0.5 & 0.3 & 1.0 & 15.0 & 7.5 & 15.0 & 0.544 \\
\hline h_Equipp & 6.3 & 4.3 & 8.3 & 0.5 & 0.3 & 1.0 & 15.0 & 7.5 & 15.0 & 0.531 \\
\hline i_Calibr & 6.3 & 4.3 & 8.3 & 0.5 & 0.3 & 1.0 & 12.0 & 6.0 & 15.0 & 0.571 \\
\hline j_Cal-EO & 5.0 & 3.0 & 7.0 & 0.5 & 0.3 & 1.0 & 12.0 & 6.0 & 15.0 & 0.596 \\
\hline k_Cal-EO-situ & 5.5 & 3.5 & 7.5 & 0.5 & 0.3 & 1.0 & 13.0 & 6.5 & 15.0 & 0.571 \\
\hline
\end{tabular}
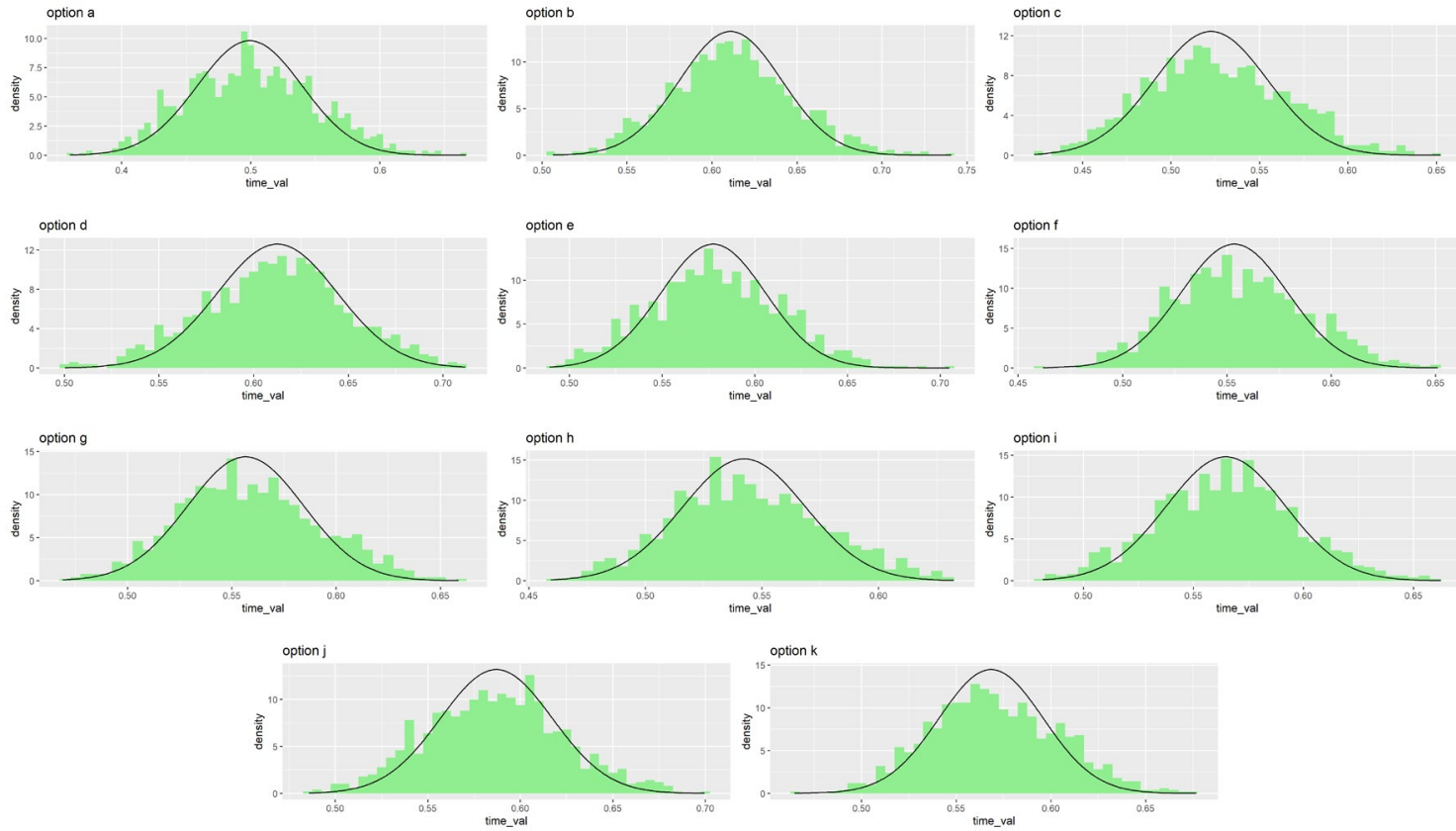

Figure SI-13: Distribution of resulting uncertainties (green) after aggregating the three sub-attributes and 1'000 Monte Carlo simulation runs. Results for the 11 FANFAR system configurations a-k. A normal distribution with mean = mean of Monte Carlo simulation, and $s d=1 / 4$ of the $95 \%$ confidence interval (black curve) fits the resulting uncertainty well. A normal distribution is thus used as input of the uncertainty of the aggregated prediction of the attribute 22 time in the subsequent MCDA. 

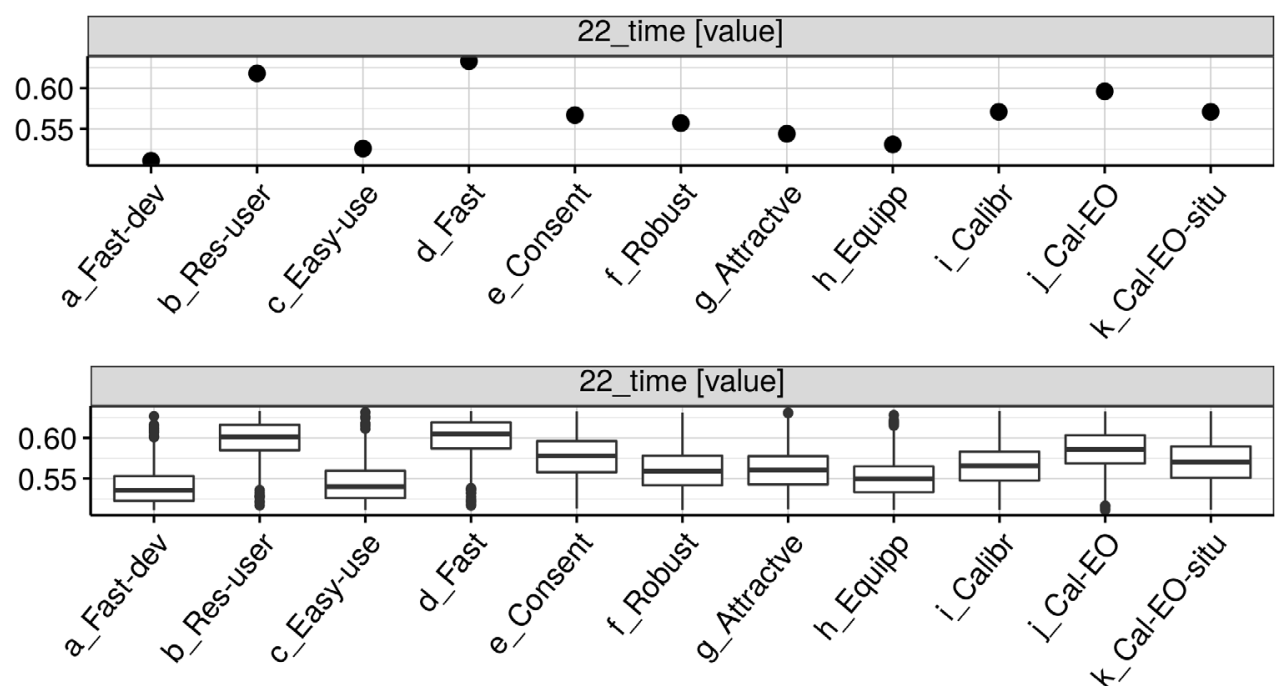

Option

Figure SI-14: Visualization of the aggregated value of predictions of all system configurations for the attribute 22_time. The plot on top represents the predictions without uncertainty, while the plot below shows the uncertainty distribution resulting from the Monte Carlo Simulation with 1'000 runs.

\subsection{Several languages}

Description: System components should be available in multiple languages. System = distribution channels (fanfar.eu, IVP, Email, SMS), support (knowledge base, forum, help desk), and Hydrology-TEP (general interface, community page, FANFAR app, processing services).

Attribute: Number of languages available for system components (23_langue)

Unit: $\quad$ score [0:17.64]

Description: Weighted sum of number of languages (En, Fr, Pt, Ar) for different system components and channels with different weights for different languages and system components; weighted sum resulting in a score.

Best case: The system and its documentations and manuals are available in English, French, Portuguese, and Arabic.

Worst case: The system and its documentations and manuals are only available in English.

\section{Attribute details:}

23_langue is an artificial (constructed) attribute calculated with a weighted sum of languages available in different system components.

Each system component can be available in several languages. Francisco Silva Pinto (Eawag) calculated the weighted sum (with different weights (w) for different languages and system components) of the number of languages available (i.e., En $[\mathrm{w}=1], \operatorname{Fr}[\mathrm{w}=0.7], \mathrm{Pt}[\mathrm{w}=0.3], \operatorname{Ar}[\mathrm{w}=0.1])$ in different system components (i.e., fanfar.eu [w=1], IVP [w=1], Email [w=1], SMS [w=1], Knowledge base [w=1], Forum [w=0.7], Help Desk [w=0.7], H-TEP General Interface [w=0.5], H-TEP Community $[\mathrm{w}=0.5]$, H-TEP FANFAR App [w=0.5], H-TEP Proc Services [w=0.5]. This weighted sum resulted in a single score of languages available for each configuration. Different levels for this attribute were created (Worst, Very Bad, Bad, Neutral, Good, Very Good, Best) which initially had a (continuous linear) level ranging from 0 (worst), over very bad (16.667), (...), to very good (83.333), and 100 (best). We later transformed the levels to a value from 0 to 1 (Table SI-16) in order to achieve a marginal value function for this attribute, which is used for the subsequent MCDA (Figure SI-15).

\section{Uncertainty of predictions:}

For this attribute there was no uncertainty in the experts' estimate. 
Table SI-16: Expert estimates for the levels of weighted sum (score) of languages for the individual system components (fanfar.eu, IVP, Email, SMS, Knowledge base, Forum, Help Desk, H-TEP General Interface, H-TEP Community, H-TEP FANFAR App, H-TEP Proc Services). Note: These levels are directly used as marginal value function in the subsequent MCDA.

\begin{tabular}{ll|l}
\hline \hline Language Level & Value & Score \\
\hline Best & 1.000 & 17.64 \\
Very Good & 0.833 & 17.10 \\
Good & 0.667 & 15.48 \\
Neutral & 0.500 & 14.58 \\
Bad & 0.333 & 10.99 \\
Very bad & 0.167 & 9.80 \\
Worst & 0.000 & 0.00 \\
\hline \hline
\end{tabular}

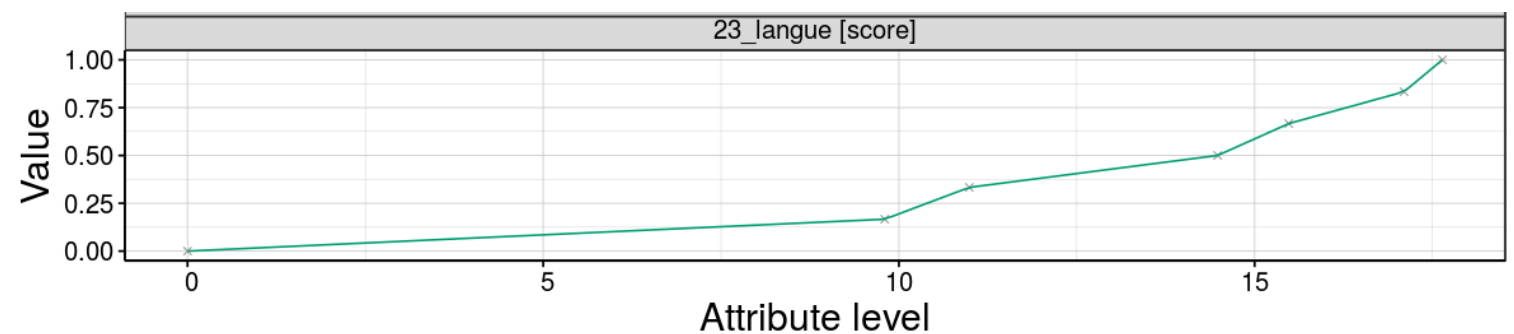

Figure SI-15: Nonlinear marginal value function for the subsequent MCDA modelling of the attribute "several languages". The shape of the value function is based on the experts' estimates on levels.

Table SI-17: Predictions on the availability of the system components in different languages. The predictions were transformed into one score for the subsequent analysis using a weighted sum with different weights for the different languages and different system components. The resulting total weighted sum (score) was directly adopted for the input of the subsequent MCDA.

\begin{tabular}{|c|c|c|c|c|c|c|c|c|c|c|c|c|}
\hline $\begin{array}{l}\text { System confi- } \\
\text { gurations }\end{array}$ & 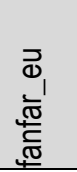 & $\stackrel{n}{\geq}$ & 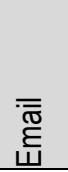 & $\sum_{\infty}^{\infty}$ & 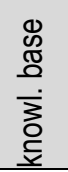 & 튼 & $\begin{array}{l}\text { के } \\
\text { ঠे } \\
\text { 을 }\end{array}$ & 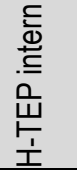 & $\begin{array}{l}\text { 응 } \\
\text { 믐 } \\
\text { I }\end{array}$ & $\begin{array}{l}\text { 응 } \\
\text { ㅁ } \\
\text { ㅁ } \\
\text { ㅁ } \\
\text { I }\end{array}$ & 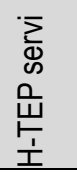 & $\begin{array}{l}\text { Total weigh- } \\
\text { ted sum } \\
\text { (score) }\end{array}$ \\
\hline a Fast-dev & efp & e & ef & e & $e$ & e & $e$ & $\mathrm{e}$ & e & $e$ & $\mathrm{e}$ & 10.10 \\
\hline b_Res-user & efpa & efpa & efpa & efpa & efpa & efpa & efpa & efpa & efpa & efpa & efpa & 17.64 \\
\hline d_Fast & efp & e & ef & e & e & e & $\mathrm{e}$ & $\mathrm{e}$ & $\mathrm{e}$ & $\mathrm{e}$ & $\mathrm{e}$ & 10.10 \\
\hline e_Consent & efpa & efpa & efpa & efpa & efpa & efpa & efpa & efp & efp & efp & efp & 17.44 \\
\hline f_Robust & efpa & efpa & efpa & efpa & efpa & efpa & efpa & efpa & efpa & efpa & efpa & 17.64 \\
\hline g_Attractve & efpa & efpa & efpa & efpa & efpa & efpa & efpa & efpa & efpa & efpa & efpa & 17.64 \\
\hline h_Equipp & efpa & efpa & efpa & efpa & efpa & efpa & efpa & efpa & efpa & efpa & efpa & 17.64 \\
\hline i_Calibr & efp & efp & efp & efp & efp & efp & efp & efp & efp & efp & efp & 16.80 \\
\hline j_Cal-EO & efp & efp & efp & efp & efp & efp & efp & efp & efp & efp & efp & 16.80 \\
\hline
\end{tabular}

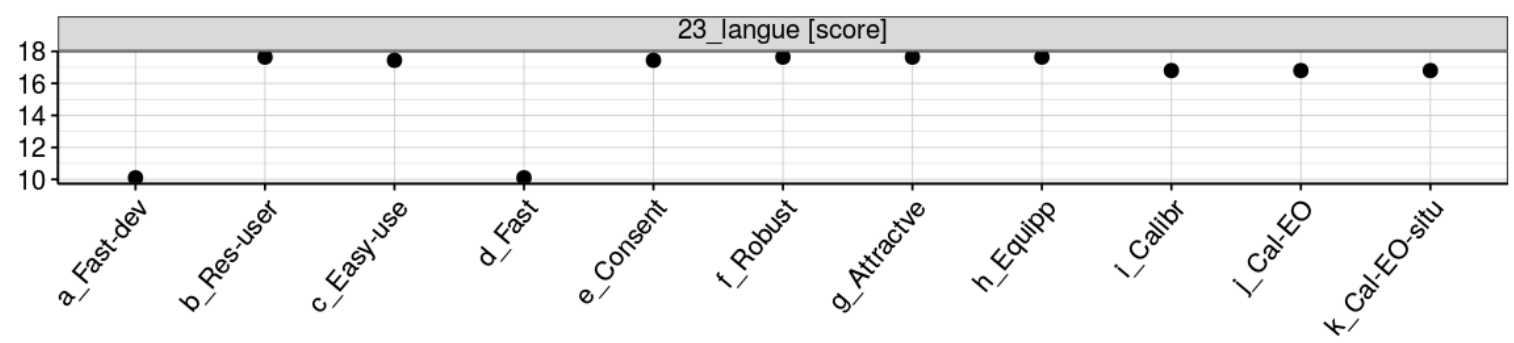

Figure SI-16: Total weighted sum of all system configurations used as predictions for the attribute 23 langue. The plot represents the predictions without uncertainty. There was no uncertainty defined for this attribute. 


\subsection{Short development time}

Description: The time required to develop the system component/ configuration. Each system configuration will demand different amount of time to develop from the FANFAR consortium.

Attribute: $\quad$ Time required to develop and implement system components (31_devlptime)

Unit: $\quad$ days [1:1095]

Description: Total time needed for the development and implementation of all technical system components in days.

Best case: 1 day is needed to develop the system component/ configuration.

Worst case: 3 years (1'095 days) are needed to develop the system component/ configuration.

\section{Attribute details:}

31_devlptime is a natural attribute directly measured in days needed for the development and implementation of all technical components.

The time required to develop and implement the individual technical system components in days was estimated by Emmanuel Mathot (Terradue) in July 2019. The time required for the development and implementation of the technical components was estimated and different components were assigned to the involved development teams at Terradue, IsardSAT, and SMHI. For each system configuration, the time needed for the technical components included in the configuration was summed up for each development team. The final development time for each configuration resulted from the maximum time requirement of the three development teams; i.e., the development takes as much time as that team needs which requires the longest time (predictions see Table SI-19). Different levels for this attribute were created (Worst, Very Bad, Bad, Neutral, Good, Very Good, Best), which initially had a (continuous linear) level ranging from 0 (worst), over very bad (16.667), (...), to very good (83.333), and 100 (best). We later transformed the levels to a value from 0 to 1 (Table SI-18) to achieve a marginal value function for this attribute for the following MCDA modelling (Figure SI-17).

\section{Uncertainty of predictions:}

The expert stated an uncertainty range around the predictions (Table SI-19). This uncertainty is included in the subsequent MCDA using a triangular distribution with mode $=$ prediction and $\mathrm{min} / \mathrm{max}=\mathrm{min} / \mathrm{max}$ estimates.

Table SI-18: Expert estimate for the levels of the value function for the attribute 3.1 Short development time. Note: These levels are directly used for the marginal value function in the subsequent MCDA.

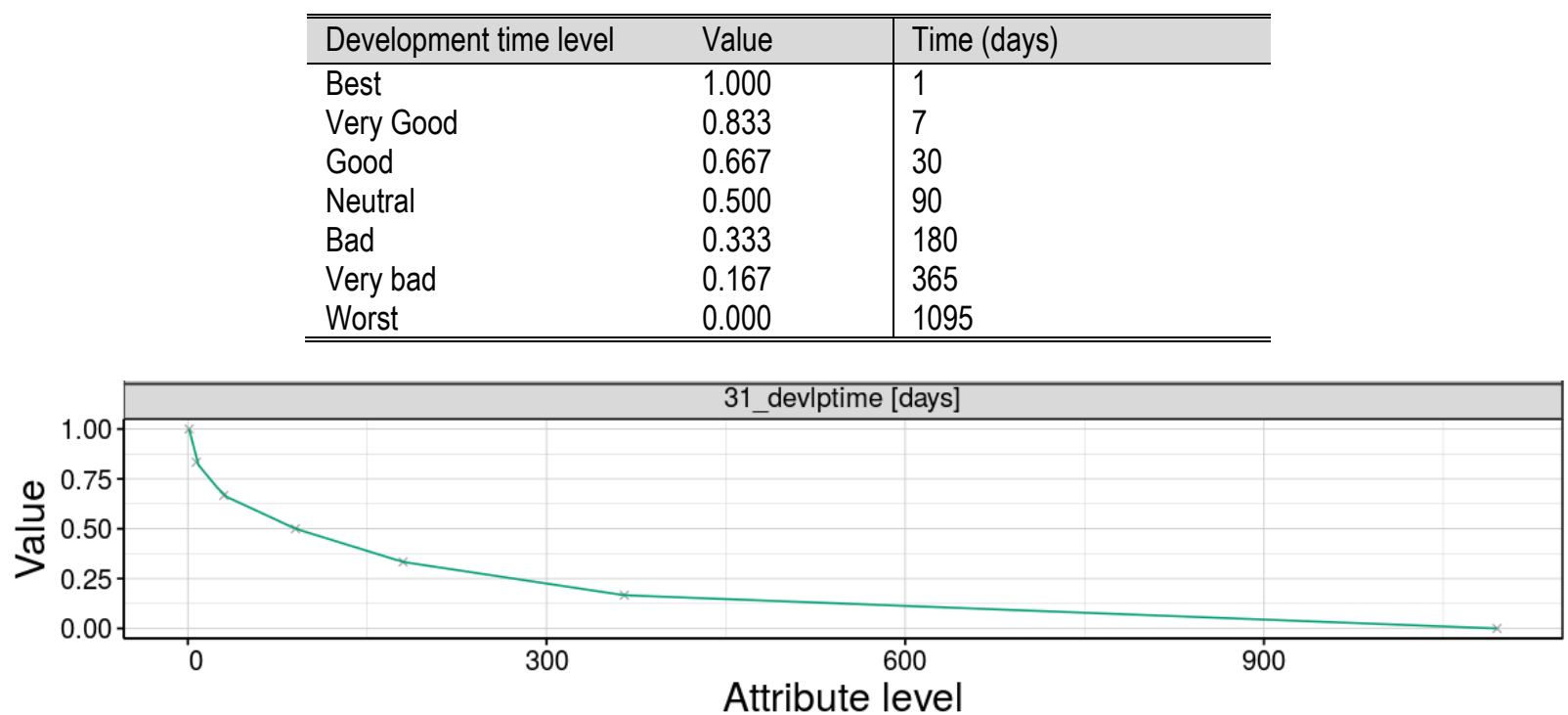

Figure SI-17: Nonlinear marginal value function for the subsequent MCDA of the attribute "short development time". The shape of the value function is based on the experts' estimates on levels ( Table SI-18). 
Table SI-19: Predictions on the development time of the system components in days for each FANFAR system configuration with $\mathrm{min} / \mathrm{max}$ values of the uncertainty range in the estimate ( $\pm 20 \%$ of prediction). The predictions (days) were directly adopted as predictions for the input of the subsequent MCDA.

\begin{tabular}{llll}
\hline System configurations & $\begin{array}{l}\text { Predicted dev. } \\
\text { Time (days) }\end{array}$ & $\begin{array}{l}\text { Min } \\
\text { (days) }\end{array}$ & $\begin{array}{l}\text { Max } \\
\text { (days) }\end{array}$ \\
\hline a_Fast-dev & 1 & 1 & 1 \\
b_Res-user & 672 & 537 & 806 \\
c_Easy-use & 600 & 480 & 720 \\
d_Fast & 278 & 222 & 333 \\
e_Consent & 1008 & 806 & 1095 \\
f_Robust & 645 & 516 & 774 \\
g_Attractve & 1008 & 806 & 1095 \\
h_Equipp & 1008 & 806 & 1095 \\
i_Calibr & 402 & 321 & 482 \\
j_Cal-EO & 432 & 345 & 518 \\
k_Cal-EO-situ & 522 & 417 & 626 \\
\hline
\end{tabular}
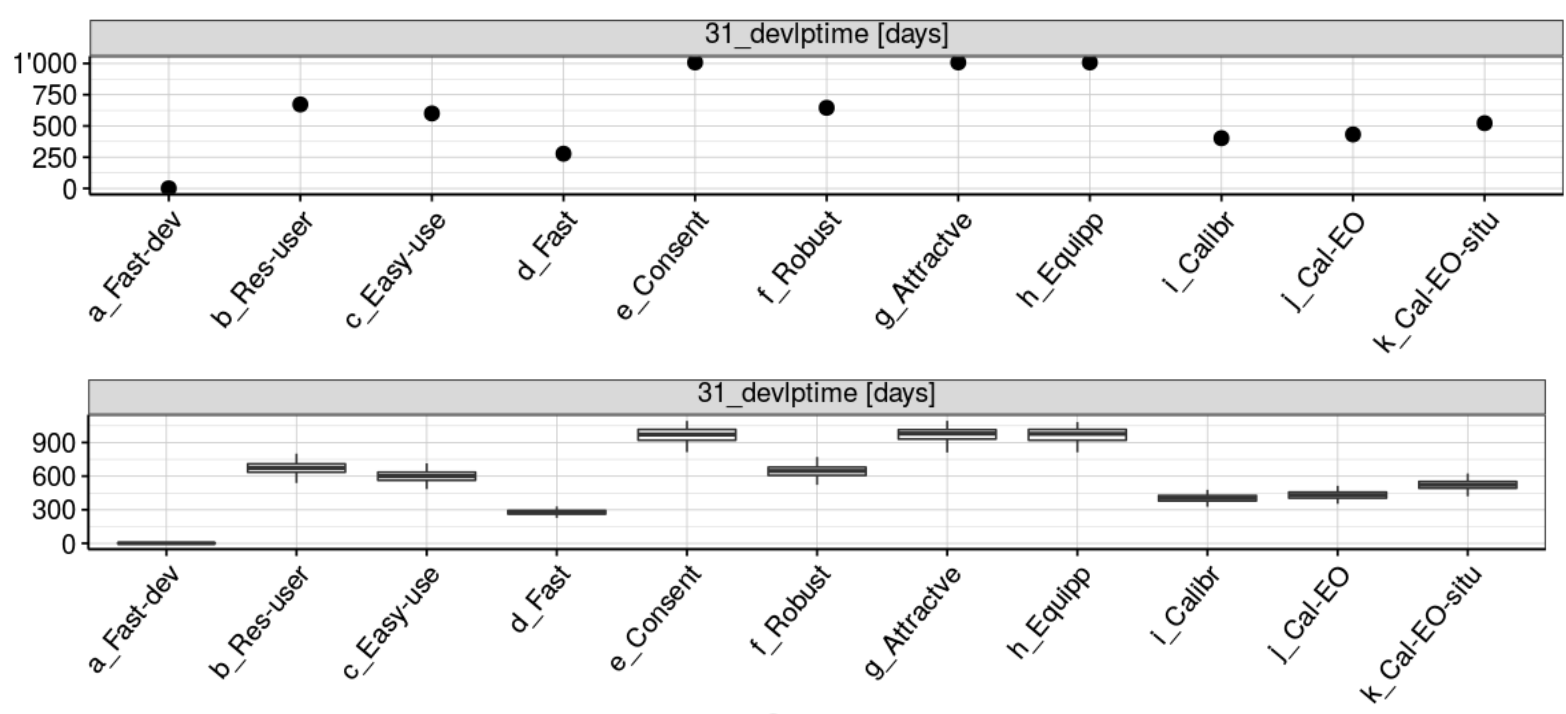

Option

Figure SI-18 Visualization of the predicted development time of all system configurations. The plot on top represents the predictions without uncertainty, while the plot below shows the uncertainty distribution resulting from the Monte Carlo Simulation with 1'000 runs.

\subsection{Low operation costs}

Description: The cost to operate and maintain the ICT infrastructure should be as low as possible (e.g., devices, networks, cloud computing resources).

Attribute: Annual operation and maintenance costs (32_costs)

Unit: $\quad$ Value [0:1]

Description: Level of annual costs to operate and maintain the system for entire West Africa.

Best case: The operation and maintenance costs are very low (e.g., 10'000 € per year, for entire West Africa).

Worst case: The operation and maintenance costs are very high (e.g., 1 million $€$ per year, for entire West Africa).

\section{Attribute details:}

31_costs is a natural attribute measured in $€ /$ year required to operate and maintain the system. For confidentiality reasons, the cost estimates of the FANFAR system configurations are not public. Therefore, the costs were transformed into a value from 0:1 representing the relative differences in estimated costs between the system configurations. 
The estimate for operation and maintenance costs are calculated based on expert estimates from Aytor Naranjo (IsardSAT), and Fabrizio Pacini (Terradue) in July 2019 for the individual system components included in each configuration. A basic minimum operation cost was estimated and the expected costs to maintain additional system components, i.e., the inclusion of observation data, and utilization of satellite data, were added depending on which components the configuration includes. Additionally, the estimated cost of the work time of the personnel needed to operate and maintain the system was added. This resulted in one final value for operation and maintenance costs for each configuration (Table SI-21). Different levels for this attribute were created (Worst, Very Bad, Bad, Neutral, Good, Very Good, Best), which initially had a continuous (linear) level ranging from 0 (worst), over very bad (16.667), (...), to very good (83.333), and 100 (best). We later transformed the levels to a value from 0 to 1 to highlight the relative performance of the system configurations for this attribute. Accordingly, the single attribute value function of this attribute in the final MCDA is linear with a range from 0:1 (Figure SI-19).

\section{Uncertainty of predictions:}

The expert stated an uncertainty range around the predictions for each component of the summed operation costs. The costs of operating a system utilizing observation data, the costs to operate satellite data, and the cost estimate on the work time needed to operate the system were each stated with a range of uncertainty. The minimum and maximum total predicted operation costs resulted from adding up the min and max values of these ranges depending on which components the system configuration includes. (see Table SI-21). This uncertainty is included in the subsequent MCDA using a triangular distribution with mode $=$ prediction and $\min / \max =$ transformed $\max / \min$ estimates.

Table SI-20: Single attribute value function of the transformed cost levels used in the subsequent MCDA.

\begin{tabular}{ll|l}
\hline \hline Cost level & Value & Level of operation costs (value) \\
\hline Best & 1.000 & 1.000 \\
Very Good & 0.833 & 0.833 \\
Good & 0.667 & 0.667 \\
Neutral & 0.500 & 0.500 \\
Bad & 0.333 & 0.333 \\
Very bad & 0.167 & 0.167 \\
Worst & 0.000 & 0.000 \\
\hline \hline
\end{tabular}

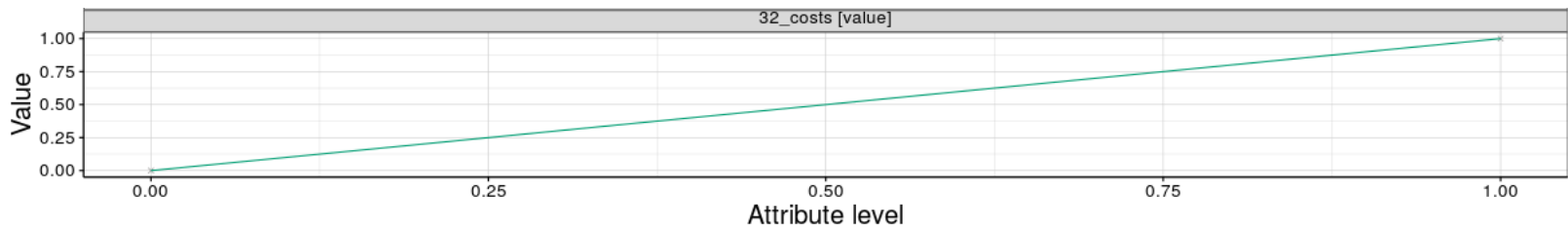

Figure SI-19: Marginal value function for the subsequent MCDA of the attribute "low operation costs". The shape of the value function is based on the experts' estimates on levels.

Table SI-21: Predictions on the values of operation costs of the system for each system configuration with min/ max values of the uncertainty range in the transformed estimate.

\begin{tabular}{llllll}
\hline $\begin{array}{l}\text { System configura- } \\
\text { tions }\end{array}$ & Observation data & Satellite Data & $\begin{array}{l}\text { Predicted opera- } \\
\text { tion costs (value) }\end{array}$ & $\begin{array}{l}\text { Min } \\
\text { (value) }\end{array}$ & $\begin{array}{l}\text { Max } \\
\text { (value) }\end{array}$ \\
\hline a_Fast-dev & No & No & 0.958 & 0.933 & 0.983 \\
b_Res-user & Yes & No & 0.468 & 0.441 & 0.495 \\
C_Easy-use & No & Yes & 0.492 & 0.472 & 0.587 \\
d_Fast & No & Yes & 0.492 & 0.472 & 0.587 \\
e_Consent & Yes & Yes & 0.451 & 0.416 & 0.486 \\
f_Robust & No & Yes & 0.492 & 0.472 & 0.587 \\
g_Attractve & Yes & Yes & 0.451 & 0.416 & 0.486 \\
h_Equipp & Yes & Yes & 0.451 & 0.416 & 0.486 \\
i_Calibr & No & No & 0.571 & 0.497 & 0.662 \\
j_Cal-EO & No & Yes & 0.492 & 0.472 & 0.587 \\
k_Cal-EO-situ & Yes & Yes & 0.451 & 0.416 & 0.486 \\
\hline
\end{tabular}



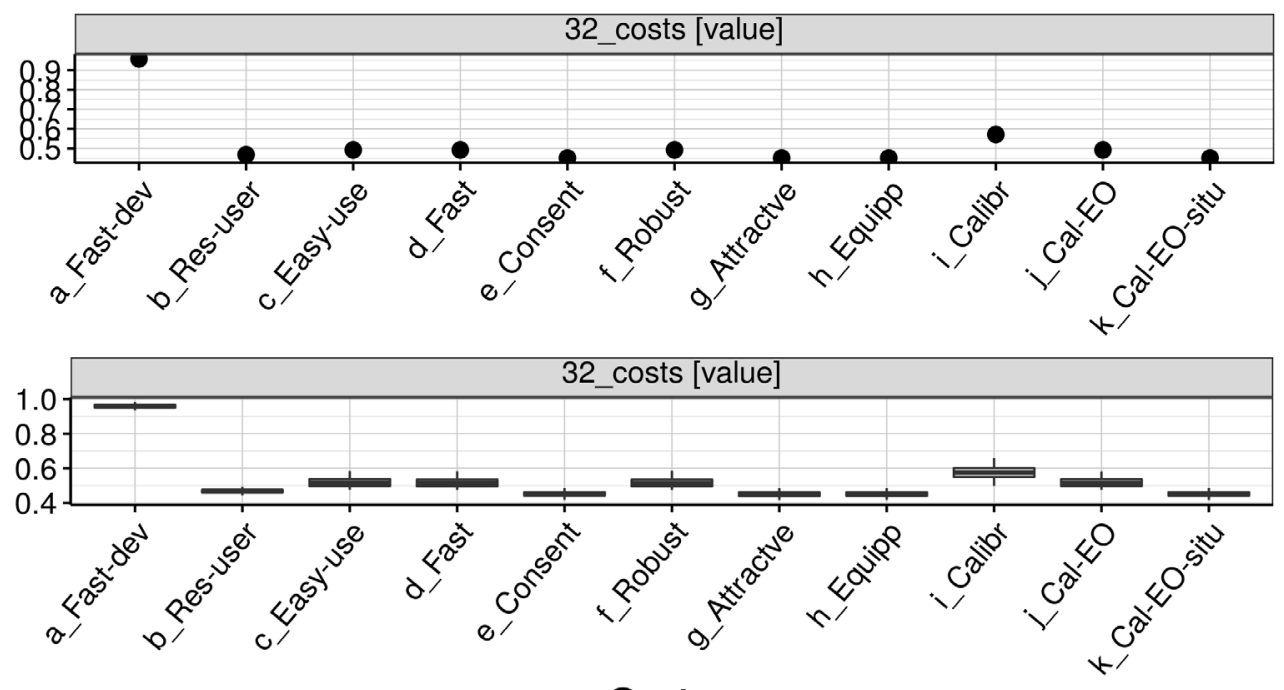

Option

Figure SI-20: Visualization of the predicted operation costs of all system configurations transformed into a 0:1 value. The plot on top represents the predictions without uncertainty, while the plot below shows the uncertainty distribution resulting from the Monte Carlo Simulation with 1'000 runs.

\subsection{Long-term financing secured}

Description: The time period for which the financing of the system is secured. This can be only for the duration of the FANFAR project, or for the entire lifespan of the system.

Attribute: $\quad$ Secured financing beyond 2020 (41_finance)

Unit: $\quad$ value [0:1]

Description: Level of secured financing beyond 2020 consisting of two sub-attributes (costs covered, and duration of financing) transformed to [0:1] value integrated with equally weighed sum.

Best case: Financing is secured for the entire lifespan of the FANFAR system (100\% for 30 years).

Worst case: No financing beyond 2020: financing ends when the FANFAR project ends.

\section{Attribute details:}

41_finance is an artificial (constructed) attribute, consisting of two sub-attributes, namely the percentage of costs covered, and the time period for which the financing of the system is secured. This can be only for the duration of the FANFAR project $(0$ years), or for the entire lifespan of the system (30 years).

The predictions for these two sub-attributes were assessed from an expert, Abdou Ali (AGRHYMET) in July 2019 (Table SI23). Seven levels were created for each sub-attribute (Worst, Very Bad, Bad, Neutral, Good, Very Good, Best), which initially had a continuous (linear) level ranging from 0 (worst), over very bad (16.667), (...), to very good (83.333), and 100 (best). We later transformed the levels to a value from 0 to 1 (Table SI-22). The two sub-attributes were then aggregated into one single value by using the levels as value functions and calculating the weighted sum with equal weight for both sub-attributes. As consequence of the transformation of the sub-attributes into one [0:1] value, a linear value function [0:1] is used for this attribute for the subsequent MCDA.

\section{Uncertainty of predictions:}

The expert stated an uncertainty range of $10 \%$ around the predictions for the first sub-attribute (costs covered). For the duration of financing, the uncertainty ranged from one year less than the prediction to maximally the prediction. This uncertainty is included in the aggregation of the two sub-attributes using a triangular distribution with $\operatorname{mode}=\operatorname{prediction}$ and $\min / \mathrm{max}=$ prediction $\pm 10 \%$. To aggregate over the two sub-attributes, we used 1'000 Monte Carlo simulation runs, each of them drawing from the triangle uncertainty distribution of each sub-attribute. For the subsequent MCDA, we used a normal distribution to model the resulting uncertainty of the two aggregated sub-attributes. To estimate the shape of the resulting uncertainty 
distribution after aggregation, we chose to utilize a normal distribution with mean = mean of Monte Carlo simulation, and standard deviation $=1 / 4$ of the $95 \%$ confidence interval resulting from the Monte Carlo simulation with 1000 runs. This resulted in an individual uncertainty distribution for each configuration for this attribute (Figure SI-22).

Table SI-22: Expert estimates for levels of the sub-attributes 1) percentage of costs covered, and 2) duration of financing secured beyond 2020 (years). These levels were used for the aggregation of the two sub-attributes into one [0:1] value.

\begin{tabular}{ll|ll}
\hline \hline Long-term financing level & Value & Costs covered & Duration of financing (years) \\
\hline Best & 1.000 & $100 \%$ & 30 \\
Very Good & 0.833 & $100 \%$ & 15 \\
Good & 0.667 & $100 \%$ & 5 \\
Neutral & 0.500 & $78 \%$ & 4 \\
Bad & 0.333 & $75 \%$ & 3 \\
Very bad & 0.167 & $70 \%$ & 1.5 \\
Worst & 0.000 & $0 \%$ & 0 \\
\hline \hline
\end{tabular}
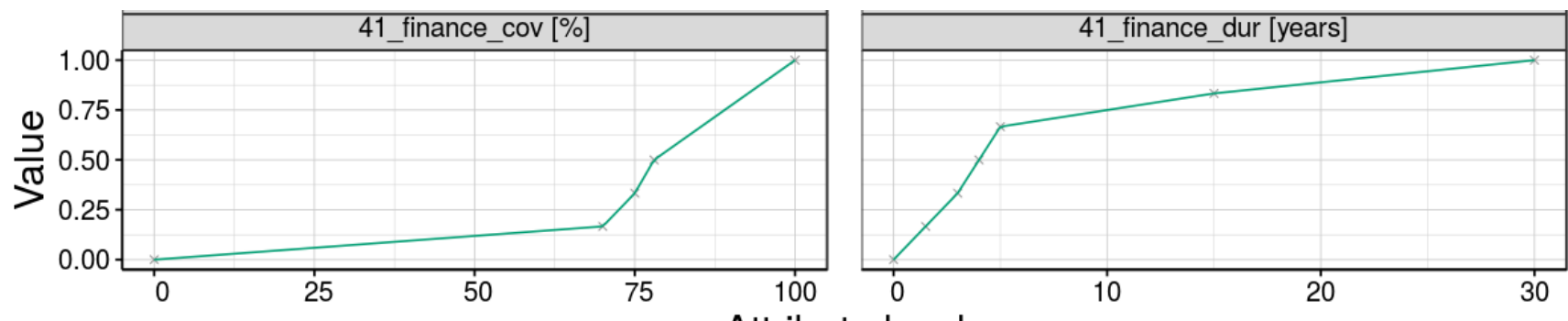

Attribute level

Figure SI-21: Value functions to aggregate the two sub-attributes into one [0:1] value. The functions were approximated from the level estimates by the expert (

Table SI-22) using linear interpolation with 14 approximated points.

Table SI-23: Predictions of the two sub-attributes costs covered, and duration of financing for each system configuration with min/ max values of uncertainty in the estimate. These predictions are expert estimates done by Abdou Ali and were aggregated into one value (last column), which is the value used as prediction for the aggregated attribute 41 finance in the subsequent MCDA modelling.

\begin{tabular}{llllllll}
\hline $\begin{array}{l}\text { System configura- } \\
\text { tions }\end{array}$ & $\begin{array}{l}\text { Predicted } \\
\text { costs cove- } \\
\text { red }\end{array}$ & Min & Max & $\begin{array}{l}\text { Predicted du- } \\
\text { ration of fi- } \\
\text { nancing } \\
\text { (years) }\end{array}$ & Min & Max & $\begin{array}{l}\text { Aggregated } \\
\text { value of pre- } \\
\text { diction }\end{array}$ \\
\hline a_Fast-dev & $30 \%$ & $27 \%$ & $33 \%$ & 1 & 0 & 1 & 0.091 \\
b_Res-user & $93 \%$ & $83 \%$ & $100 \%$ & 4 & 3 & 4 & 0.670 \\
c_Easy-use & $83 \%$ & $74 \%$ & $91 \%$ & 5 & 4 & 5 & 0.640 \\
d_Fast & $90 \%$ & $81 \%$ & $99 \%$ & 3 & 2 & 3 & 0.553 \\
e_Consent & $95 \%$ & $85 \%$ & $100 \%$ & 5 & 4 & 5 & 0.777 \\
f_Robust & $89 \%$ & $80 \%$ & $97 \%$ & 5 & 4 & 5 & 0.708 \\
g_Attractve & $98 \%$ & $88 \%$ & $100 \%$ & 5 & 4 & 5 & 0.811 \\
h_Equipp & $84 \%$ & $75 \%$ & $92 \%$ & 6 & 5 & 6 & 0.660 \\
i_Calibr & $95 \%$ & $85 \%$ & $100 \%$ & 3 & 2 & 3 & 0.610 \\
j_Cal-EO & $96 \%$ & $86 \%$ & $100 \%$ & 4 & 3 & 4 & 0.705 \\
k_Cal-EO-situ & $86 \%$ & $77 \%$ & $94 \%$ & 5 & 4 & 5 & 0.674 \\
\hline
\end{tabular}
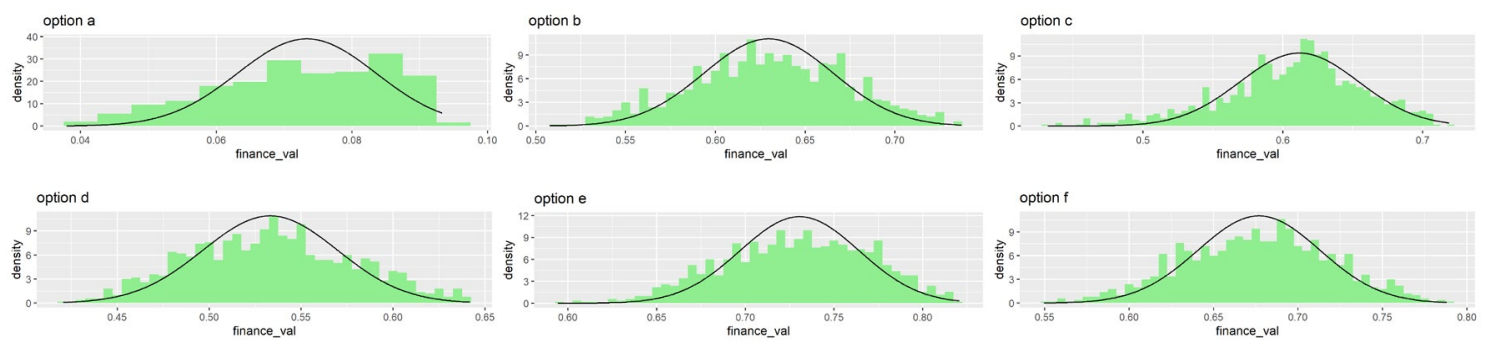


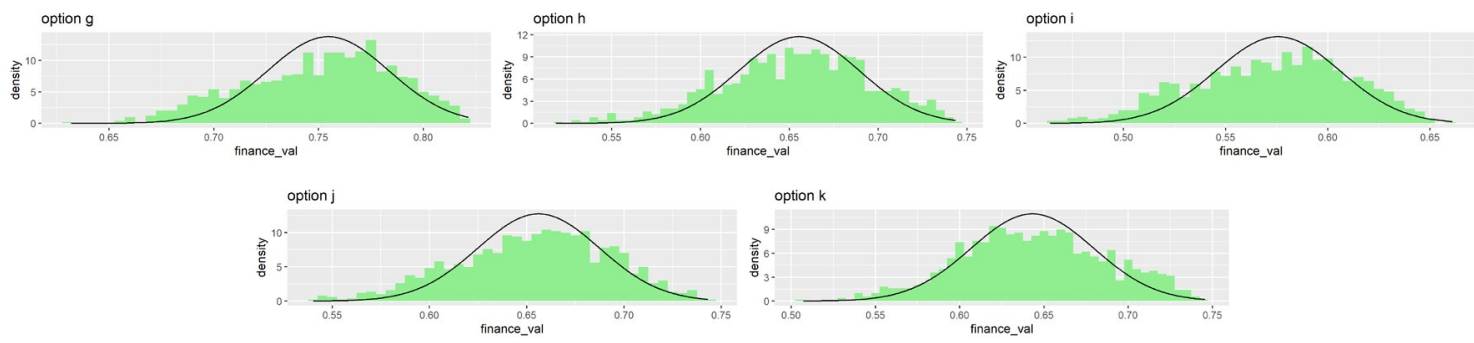

Figure SI-22: Distribution of resulting uncertainties (green) after aggregating the three sub-attributes and 1'000 Monte Carlo simulation runs. Results for the 11 FANFAR system configurations a $-k$. A normal distribution with mean = mean of Monte Carlo simulation, and $s d=1 / 4$ of the $95 \%$ confidence interval (black curve) fits the resulting uncertainty well. A normal distribution is thus used as input of the uncertainty of the aggregated prediction of the attribute 41_finance in the subsequent MCDA.
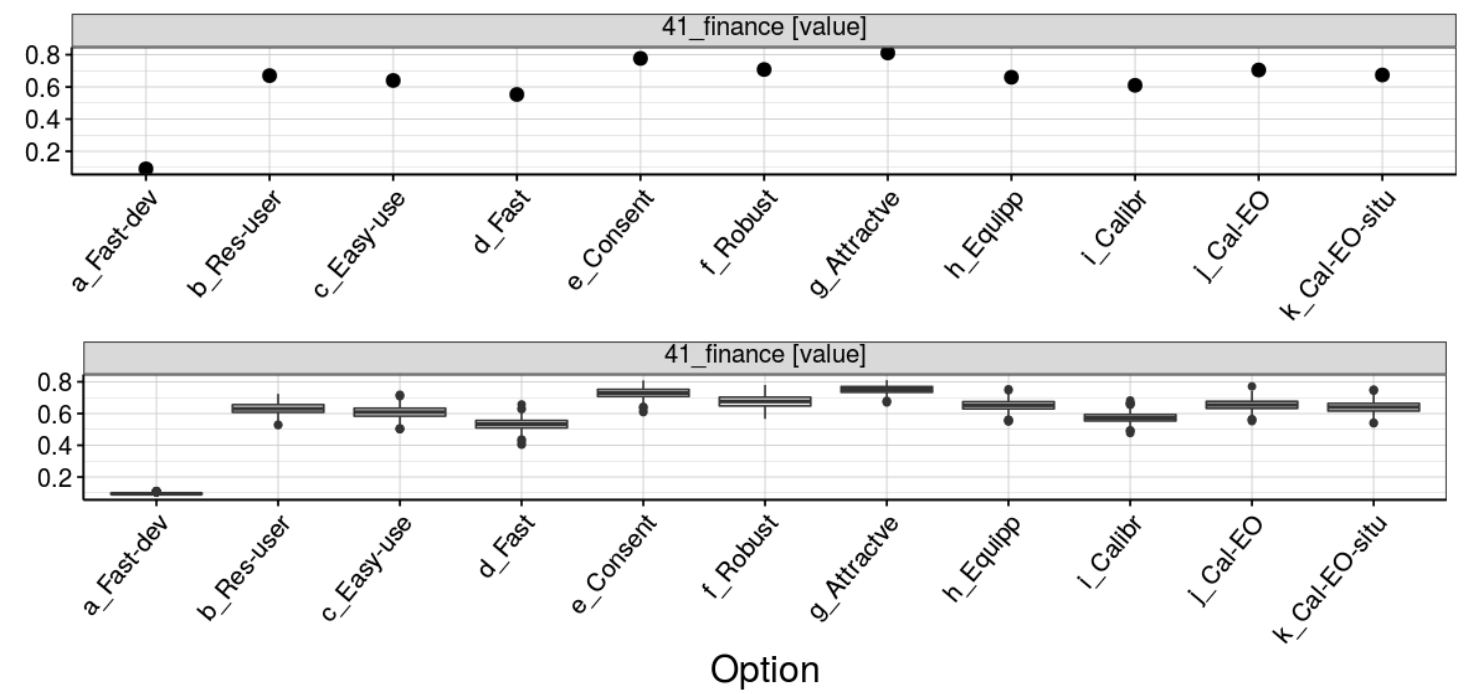

Figure SI-23: Visualization of the aggregated value of predictions of all system configurations for the attribute 41_finance. The plot on top represents the predictions without uncertainty, while the plot below shows the uncertainty distribution resulting from the Monte Carlo Simulation with 1'000 runs.

\subsection{Skillful human resources available}

Description: At the end of the FANFAR project (and included training), the availability of skilled staff at West African institutions that are able to access, interpret, operate, maintain, and develop the forecasting and alert system.

Attribute: $\quad$ Number of people in $W$ Africa for development, $O \& M$, and access \& interpretation (42_experts)

Unit: $\quad$ value [0:1]

Description: Level of skillful human resources available of three sub-attributes (number of people in West Africa available to 1) develop \& maintain, 2) operate, and 3) access \& interpret the system) transformed to a [0:1] value aggregated with equally weighted sum.

Best case: 5 persons are able to develop and maintain the system, 10 to operate it. 100 persons are able to access and interpret the forecasts and flood risk notifications (at least 3 in each country).

Worst case: Nobody is able to develop, maintain, and operate the forecasting system, and nobody is able to access and interpret its outputs.

\section{Attribute details:}

42 experts is an artificial (constructed) attribute consisting of three sub-attributes, namely the number of persons: 1) able to develop and maintain the system, 2) able to operate it, and 3) able to access and interpret the forecasts and flood risk notifications.

To predict the outcomes of the individual FANFAR system configurations, we used expert estimates. These were elicited from Jafet Andersson (SMHI, hydrologist from FANFAR) in July 2019. He estimated the number of people able to 1) develop and 
maintain, 2) able to operate, and 3) able to access and interpret the forecasts and flood risk notifications for each system configuration (predictions see Table SI-25). Seven levels were created for each sub-attribute (Worst, Very Bad, Bad, Neutral, Good, Very Good, Best), which initially had a (continuous linear) level ranging from 0 (worst), over very bad (16.667), (...), to very good (83.333), and 100 (best). We later transformed the levels to a value from 0 to 1 (Table SI-24). These three subattributes were transformed to one single [0:1] value using nonlinear value functions based on the estimated levels, and aggregated with a weighted sum, where each sub-attribute received the same weight of 0.33 . As consequence of the transformation of the sub-attributes into one [0:1] value, a linear value function [0:1] is used for this attribute for the subsequent MCDA modelling.

\section{Uncertainty of predictions:}

For each sub-attribute, the expert gave a different uncertainty range. For the estimate of people able to develop and maintain the system, he stated an uncertainty of $10 \%$ around his prediction. The prediction of people able to operate it lies within an uncertainty range of $20 \%$ around the predicted number. And with $40 \%$ around the prediction, even more uncertainty was expected in the estimate of how many people are able to access and interpret the system. The expert initially stated uncertainty ranges exceeding the value function range. These uncertainty ranges were trimmed off to lie within the value function, as any predictions outside the value function range will achieve a value of 0 (if undershooting) or 1 (if overshooting). The uncertainty ranges were included in the aggregation of the three sub-attributes using a triangular distribution with mode $=$ prediction, and $\min / \max =\min / \max$ of stated uncertainty range. To aggregate over the three sub-attributes, we used 1'000 Monte Carlo simulation runs, each of them drawing from the triangle uncertainty distribution of each sub-attribute. For the subsequent MCDA, we used a normal distribution to model the aggregated uncertainty of the three sub-attributes. To estimate the shape of the aggregated uncertainty distribution, we chose to utilize a normal distribution with mean = mean of Monte Carlo simulation, and standard deviation $=1 / 4$ of the $95 \%$ confidence interval resulting from the Monte Carlo simulation with 1 '000 runs. This resulted in an individual uncertainty distribution for each configuration for this attribute (Figure SI-25).

Table SI-24: Expert estimates for levels of the three sub-attributes: people able to 1) develop and maintain, 2) operate, and 3) access and interpret the FANFAR system. These levels were used to shape the value functions for aggregating the three sub-attributes.

\begin{tabular}{ll|lll}
\hline \hline Human resource level & Value & $\begin{array}{l}\text { People able to develop } \\
\text { and maintain }\end{array}$ & $\begin{array}{l}\text { People able to operate } \\
\text { Best }\end{array}$ & $\begin{array}{l}\text { People able to access } \\
\text { and interpret }\end{array}$ \\
\hline Very Good & 1.000 & 5 & 10 & 100 \\
Good & 0.833 & 3 & 7 & 80 \\
Neutral & 0.667 & 2 & 5 & 60 \\
Bad & 0.500 & 1 & 3 & 40 \\
Very bad & 0.333 & 0 & 1 & 20 \\
Worst & 0.167 & 0 & 0 & 5 \\
\hline \hline
\end{tabular}

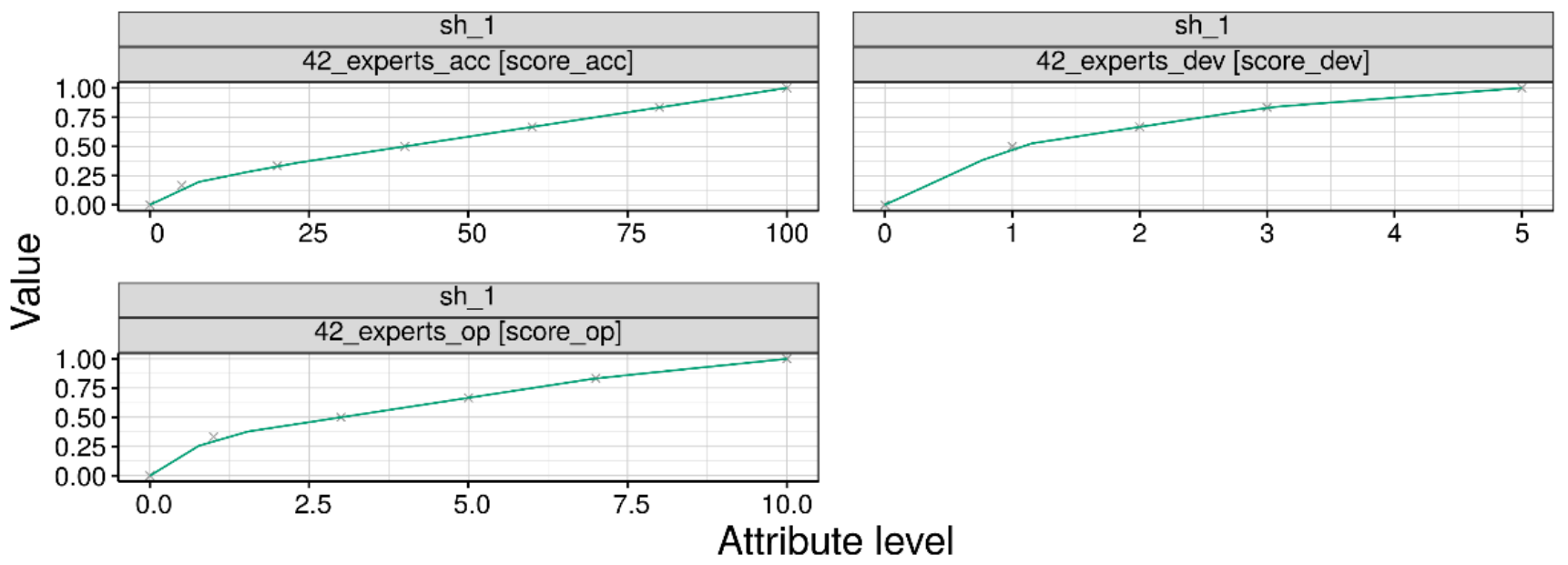

Figure SI-24: Value functions to aggregate the three sub-attributes into one [0:1] value. The functions were approximated from the level estimates by the expert using linear interpolation with 14 approximated points. 
Table SI-25: Predictions of the three sub-attributes people able to 1) develop and maintain the system, 2) operate the system, and 3) access and interpret the forecasts and notifications for each system configuration with min/ max values of uncertainty in the estimate. These predictions are expert estimates done by Jafet Andersson and were aggregated into one value (last column), which is the value used as prediction for the aggregated attribute 42_experts in the subsequent MCDA modelling.

\begin{tabular}{|c|c|c|c|c|c|c|c|c|c|c|}
\hline $\begin{array}{l}\text { System confi- } \\
\text { gurations }\end{array}$ & $\begin{array}{l}\text { Predicted } \\
\text { people able } \\
\text { to develop } \\
\text { \& maintain }\end{array}$ & Min & Max & $\begin{array}{l}\text { Predicted } \\
\text { people } \\
\text { able to op- } \\
\text { erate }\end{array}$ & Min & Max & $\begin{array}{l}\text { Predicted } \\
\text { people able } \\
\text { to access \& } \\
\text { interpret }\end{array}$ & Min & Max & $\begin{array}{l}\text { Aggregated } \\
\text { value of } \\
\text { prediction }\end{array}$ \\
\hline a_Fast-dev & 3 & 2 & 4 & 5 & 4 & 6 & 50 & 30 & 70 & 0.694 \\
\hline b_Res-user & 3 & 2 & 4 & 3 & 2 & 4 & 90 & 54 & 100 & 0.750 \\
\hline c_Easy-use & 2 & 1 & 3 & 4 & 3 & 5 & 80 & 48 & 100 & 0.694 \\
\hline d_Fast & 1 & 0 & 2 & 4 & 3 & 5 & 70 & 42 & 98 & 0.611 \\
\hline e_Consent & 0 & 0 & 0 & 6 & 4 & 8 & 90 & 54 & 100 & 0.556 \\
\hline f_Robust & 2 & 1 & 3 & 7 & 5 & 9 & 90 & 54 & 100 & 0.806 \\
\hline g_Attractve & 0 & 0 & 0 & 6 & 4 & 8 & 90 & 54 & 100 & 0.556 \\
\hline h_Equipp & 0 & 0 & 0 & 6 & 4 & 8 & 100 & 60 & 100 & 0.583 \\
\hline i_Calibr & 4 & 3 & 5 & 7 & 5 & 9 & 90 & 54 & 100 & 0.889 \\
\hline j_Cal-EO & 4 & 3 & 5 & 6 & 4 & 8 & 90 & 54 & 100 & 0.861 \\
\hline k_Cal-EO-situ & 4 & 3 & 5 & 5 & 4 & 6 & 90 & 54 & 126 & 0.833 \\
\hline
\end{tabular}
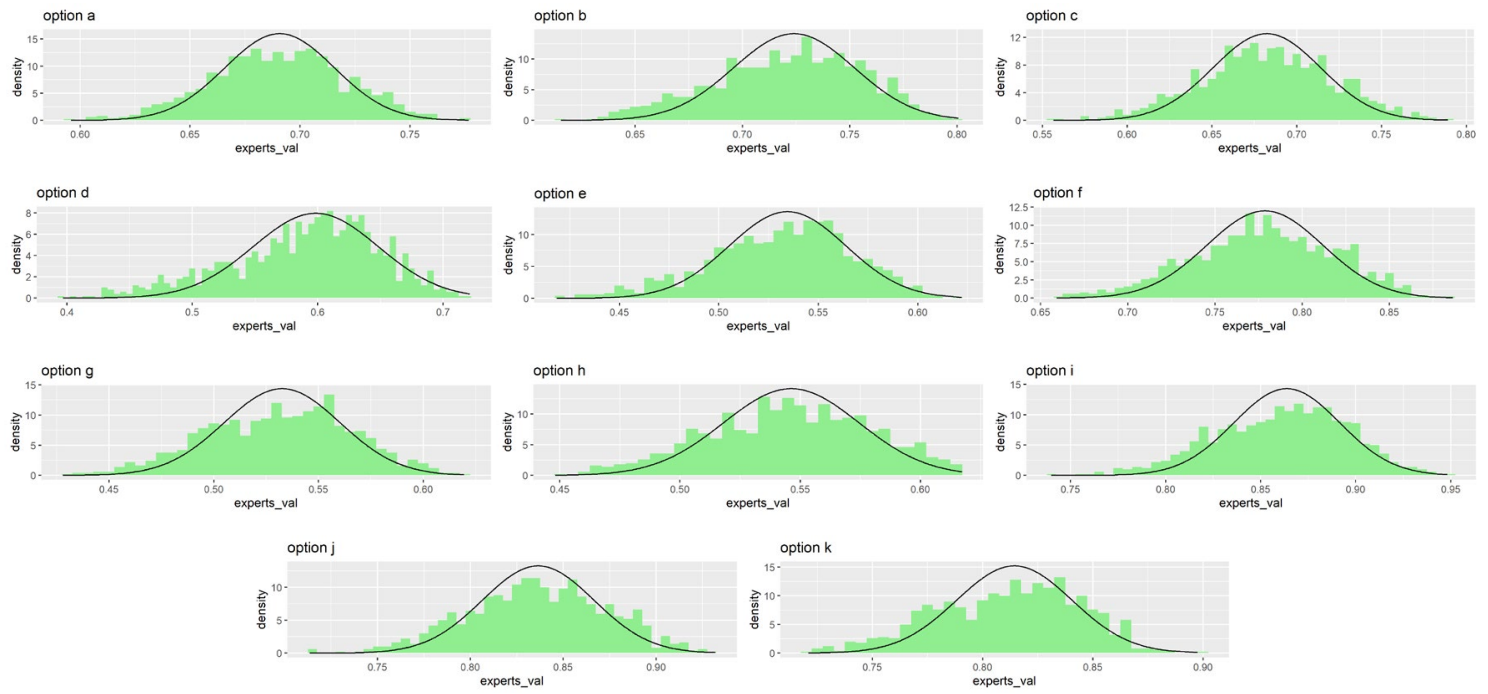

Figure SI-25: Distribution of resulting uncertainties (green) after aggregating the three sub-attributes and 1'000 Monte Carlo simulation runs. Results for the 11 FANFAR system configurations a $-k$. A normal distribution with mean = mean of Monte Carlo simulation, and $s d=1 / 4$ of the $95 \%$ confidence interval (black curve) fits the resulting uncertainty well. A normal distribution is thus used as input of the uncertainty of the aggregated prediction of the attribute 42_experts in the subsequent MCDA.
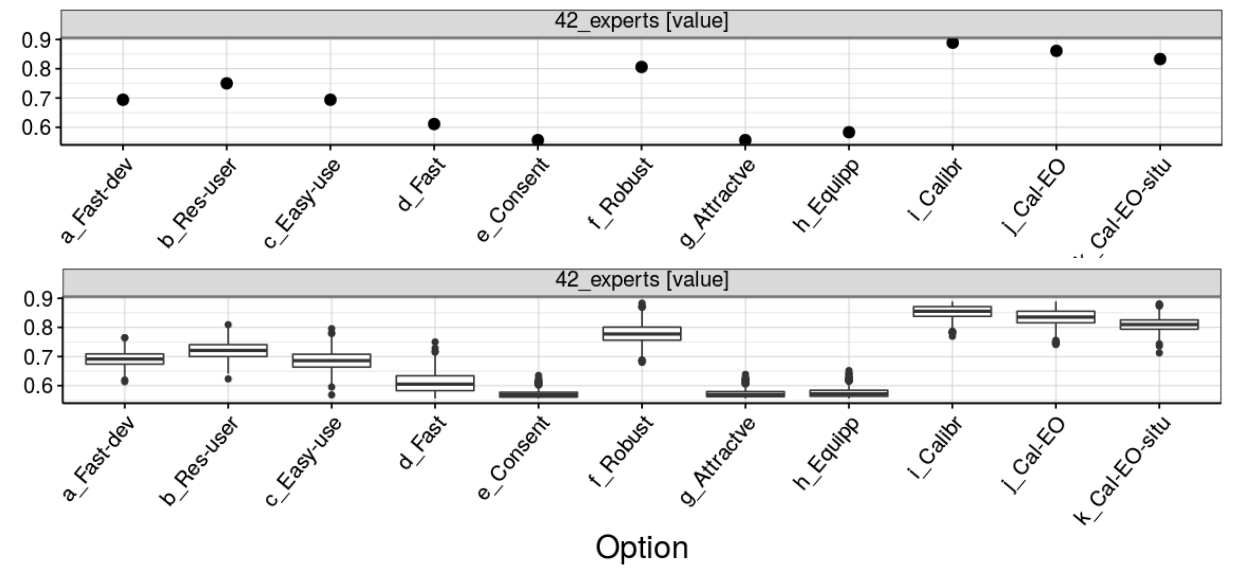

Figure SI-26: Aggregated value of predictions of all system configurations for the attribute 42 experts. The plot on top represents the predictions without uncertainty, the plot below shows the uncertainty distribution resulting from 1'000 Monte Carlo Simulation runs. 


\subsection{Good support system}

Description: The support system should quickly help users with any questions or problems they have regarding fanfar.eu, IVP, Email, SMS, Knowledge base, Forum, Help Desk, H-TEP General Interface, H-TEP Community, H-TEP FANFAR App, and H-TEP Proc Services.

Attribute: Information quantity in support system and time of response to resolve issues (43_suppsys)

Unit: $\quad$ value [0:1]

Description: Level of support system quality consisting of two sub-attributes (1) Information quantity, and 2) time for response of support system; transformed into a [0:1] value aggregated with equally weighted sum.

Best case: The support system is informative (instructive answers to all issues are available in the knowledge base or forum, Score $=6.8$ ). The support team responds quickly to questions posed (1 hour).

Worst case: The support system is not informative. The support team does not react when there are queries and does not help to solve problems.

\section{Attribute details:}

43 suppsys is an artificial (constructed) attribute consisting of two sub-attributes: 1) quantity of information available, and 2) time of response to solve issues.

For sub-attribute 1, we calculated a weighted sum to achieve a single score of the availability of information for each configuration. In this weighted sum, we assigned different weights (w) for different system components of the estimated information available in the knowledge base (i.e., fanfar.eu [w=0.3], IVP [w=1.75], Email [w=0.8], SMS [w=0.8], Knowledge base $[w=1]$, Forum [w=0.5], Help Desk [w=0.5], H-TEP General Interface [w=0.5], H-TEP Community [w=0.1], H-TEP FANFAR App [w=0.7], and H-TEP Proc Services [w=0.6]). The estimates were elicited from Aytor Naranjo (IsardSAT) in July 2019. For the second sub-attribute, Aytor Naranjo estimated the minimum and maximum time required for a response to resolve issues. The average from these two estimates formed the prediction (Table SI-27). Levels were created for each subattribute (Worst, Very Bad, Bad, Neutral, Good, Very Good, Best; Table SI-26). These levels were then used as value functions for the aggregation of the two sub-attributes into one final value [0-1] using the weighted sum with equal weights for both subattributes (Figure SI-27). As consequence of the transformation of the sub-attributes into one [0:1] value, a linear value function $[0: 1]$ is used for this attribute for the subsequent MCDA modelling.

\section{Uncertainty of predictions:}

For the first sub-attribute "Quantity of information available", there was no uncertainty in the estimate. For the second subattribute "Time of response", minimum and maximum values were estimated, which were then averaged to calculate the prediction. The minimum and maximum times of response were included as uncertainty range in the aggregation of the two subattributes using a triangular distribution with mode $=$ prediction, and $\mathrm{min} / \mathrm{max}=\mathrm{min} / \mathrm{max}$ estimated time. To aggregate over the two sub-attributes, we used the fixed predictions for sub-attribute 1), and 1'000 Monte Carlo simulation runs, each of them drawing from the triangle uncertainty distribution of sub-attribute 2). For the subsequent MCDA, we used a normal distribution to model the resulting uncertainty of aggregated values. To estimate the shape of the aggregated uncertainty distribution, we chose to utilize a normal distribution with mean = mean of Monte Carlo simulation, and standard deviation $=1 / 4$ of the $95 \%$ confidence interval resulting from the Monte Carlo simulation with 1000 runs. This resulted in an individual uncertainty distribution for each configuration for this attribute (Figure SI-28).

Table SI-26: Expert estimates for levels of the two sub-attributes 1) quantity of information available, and 2) time of response to resolve issues. These levels were used to shape the value functions for the aggregation of the two sub-attributes into one [0:1] value.

\begin{tabular}{ll|ll}
\hline \hline $\begin{array}{l}\text { Support system } \\
\text { level }\end{array}$ & Value & $\begin{array}{l}\text { Quantity of information available } \\
\text { (score) }\end{array}$ & $\begin{array}{l}\text { Time of response } \\
(\mathrm{h})\end{array}$ \\
\hline Best & 1.000 & 6,8 & 1 \\
Very Good & 0.833 & 5,7 & 6 \\
Good & 0.667 & 4,5 & 24 \\
Neutral & 0.500 & 3,4 & 72 \\
Bad & 0.333 & 2,3 & 168 \\
Very bad & 0.167 & 1,1 & 720 \\
Worst & 0.000 & 0,0 & $26^{\prime} 280$ \\
\hline \hline
\end{tabular}



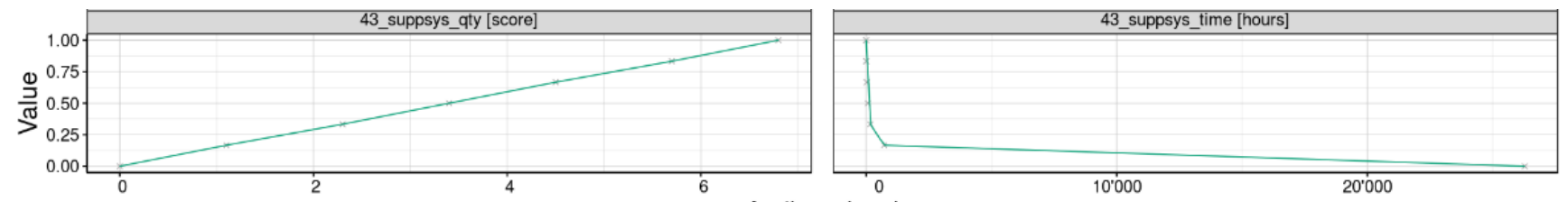

Figure SI-27: Value functions used to aggregate the two sub-attributes into one [0:1] value. The functions were approximated from the level estimates by the expert using linear interpolation with 140 approximated points.

Table SI-27: Predictions on: 1) the availability of information for different system components. These predictions were transformed into one score for the subsequent analysis using a weighted sum with different weights for the different system components. Predictions on: 2) the time of required for a response to resolve issues. These predictions result from averaging the min/max estimates by the expert. The total summed score and the predicted time of response were aggregated into one [0:1] value for the aggregated attribute 43_suppsys in the subsequent MCDA modelling (last column).

\begin{tabular}{|c|c|c|c|c|c|c|c|c|c|c|c|c|c|c|c|c|}
\hline $\begin{array}{l}\text { System configu- } \\
\text { rations }\end{array}$ & 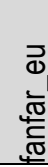 & $\stackrel{\cap}{\geq}$ & $\begin{array}{l}\overline{\overline{\widetilde{E}}} \\
\text { U్ }\end{array}$ & $\sum_{\infty}^{\infty}$ & 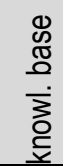 & 톨 & $\begin{array}{l}\text { 흠 } \\
\frac{0}{0} \\
\text { 을 } \\
\end{array}$ & 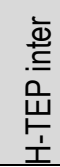 & $\begin{array}{l}\varepsilon \\
\text { ㅇ } \\
\text { 口 } \\
\stackrel{1}{I} \\
\end{array}$ & $\begin{array}{l}\text { 음 } \\
\text { 㟧 } \\
\stackrel{1}{1}\end{array}$ & 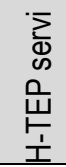 & $\begin{array}{l}\text { Total } \\
\text { summed } \\
\text { score }\end{array}$ & $\begin{array}{l}\text { Predicted } \\
\text { time of } \\
\text { response }\end{array}$ & Min & Max & $\begin{array}{l}\text { Aggregated } \\
\text { value of pre- } \\
\text { diction }\end{array}$ \\
\hline a_Fast-dev & 1 & 1 & 0 & 0 & 1 & 0 & 0 & 1 & 1 & 1 & 1 & 4.46 & 2.50 & 1.0 & 4.0 & 0.805 \\
\hline b_Res-user & 1 & 1 & 1 & 1 & 1 & 1 & 0 & 1 & 1 & 1 & 1 & 6.35 & 2.50 & 1.0 & 4.0 & 0.941 \\
\hline c_Easy-use & 1 & 1 & 0 & 0 & 1 & 1 & 0 & 1 & 1 & 1 & 1 & 4.91 & 2.50 & 1.0 & 4.0 & 0.837 \\
\hline d_Fast & 1 & 1 & 1 & 1 & 1 & 1 & 1 & 1 & 1 & 1 & 1 & 6.80 & 2.25 & 0.5 & 4.0 & 0.979 \\
\hline e_Consent & 1 & 1 & 1 & 1 & 1 & 1 & 1 & 1 & 1 & 1 & 1 & 6.80 & 2.25 & 0.5 & 4.0 & 0.979 \\
\hline f_Robust & 1 & 1 & 1 & 1 & 1 & 1 & 1 & 1 & 1 & 1 & 1 & 6.80 & 2.25 & 0.5 & 4.0 & 0.979 \\
\hline g_Attractve & 1 & 1 & 1 & 1 & 1 & 1 & 1 & 1 & 1 & 1 & 1 & 6.80 & 2.25 & 0.5 & 4.0 & 0.979 \\
\hline h_Equipp & 1 & 1 & 1 & 1 & 1 & 1 & 1 & 1 & 1 & 1 & 1 & 6.80 & 2.25 & 0.5 & 4.0 & 0.979 \\
\hline i_Calibr & 1 & 1 & 1 & 1 & 1 & 1 & 1 & 1 & 1 & 1 & 1 & 6.80 & 2.50 & 1.0 & 4.0 & 0.975 \\
\hline j_Cal-EO & 1 & 1 & 1 & 1 & 1 & 1 & 1 & 1 & 1 & 1 & 1 & 6.80 & 2.50 & 1.0 & 4.0 & 0.975 \\
\hline k_Cal-EO-situ & 1 & 1 & 1 & 1 & 1 & 1 & 1 & 1 & 1 & 1 & 1 & 6.80 & 2.50 & 1.0 & 4.0 & 0.975 \\
\hline
\end{tabular}
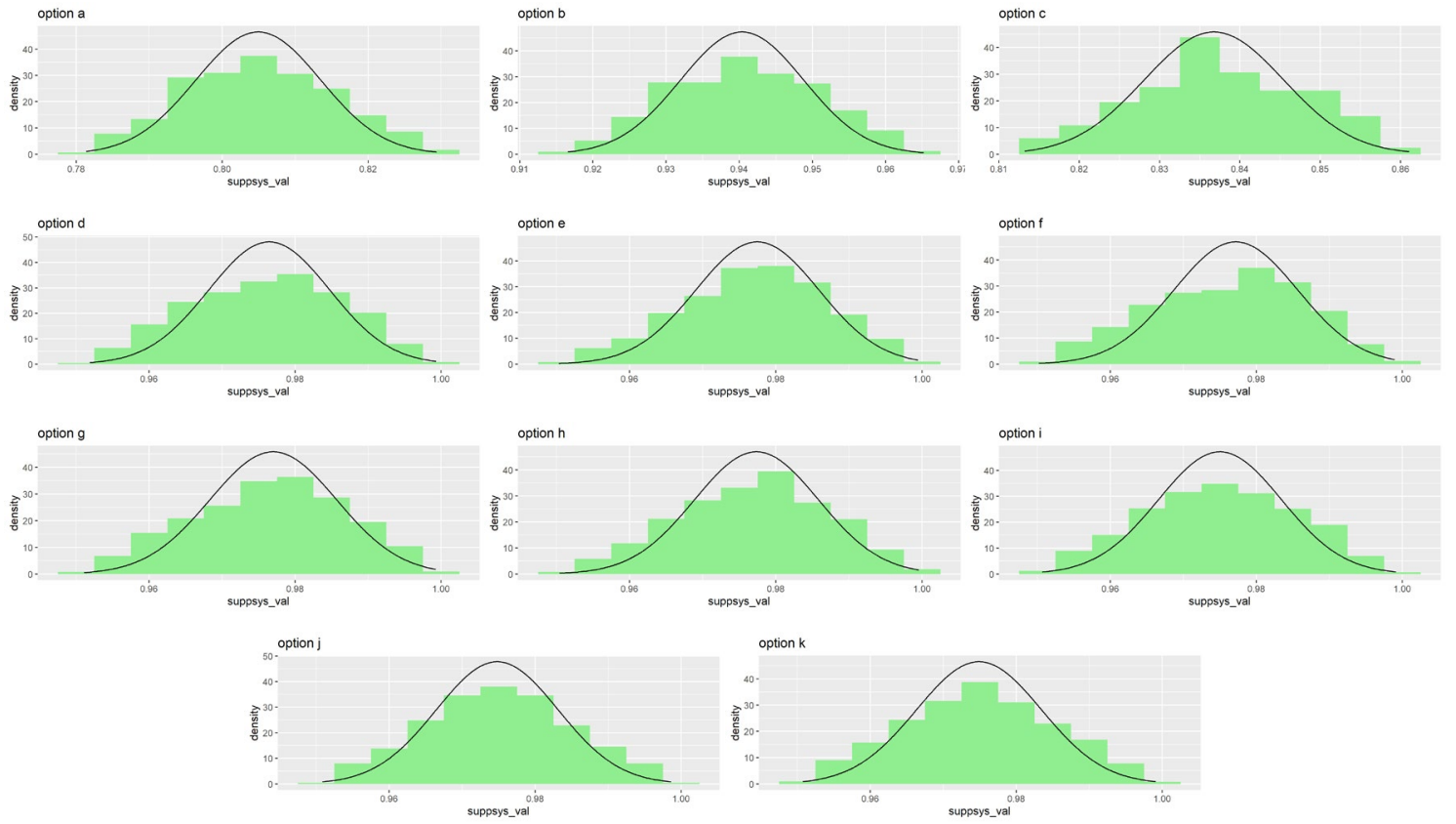

Figure SI-28: Distribution of resulting uncertainties (green) after aggregating the two sub-attributes and 1'000 Monte Carlo simulation runs. Results for the 11 FANFAR system configurations $\mathbf{a}-\mathbf{k}$. A normal distribution with mean $=$ mean of Monte Carlo simulation, and $\mathbf{s d}=1 / 4$ of the $95 \%$ confidence interval (black curve) fits the resulting uncertainty well. A normal distribution is thus used as input of the uncertainty of the aggregated prediction of the attribute 43 suppsys in the subsequent MCDA. 

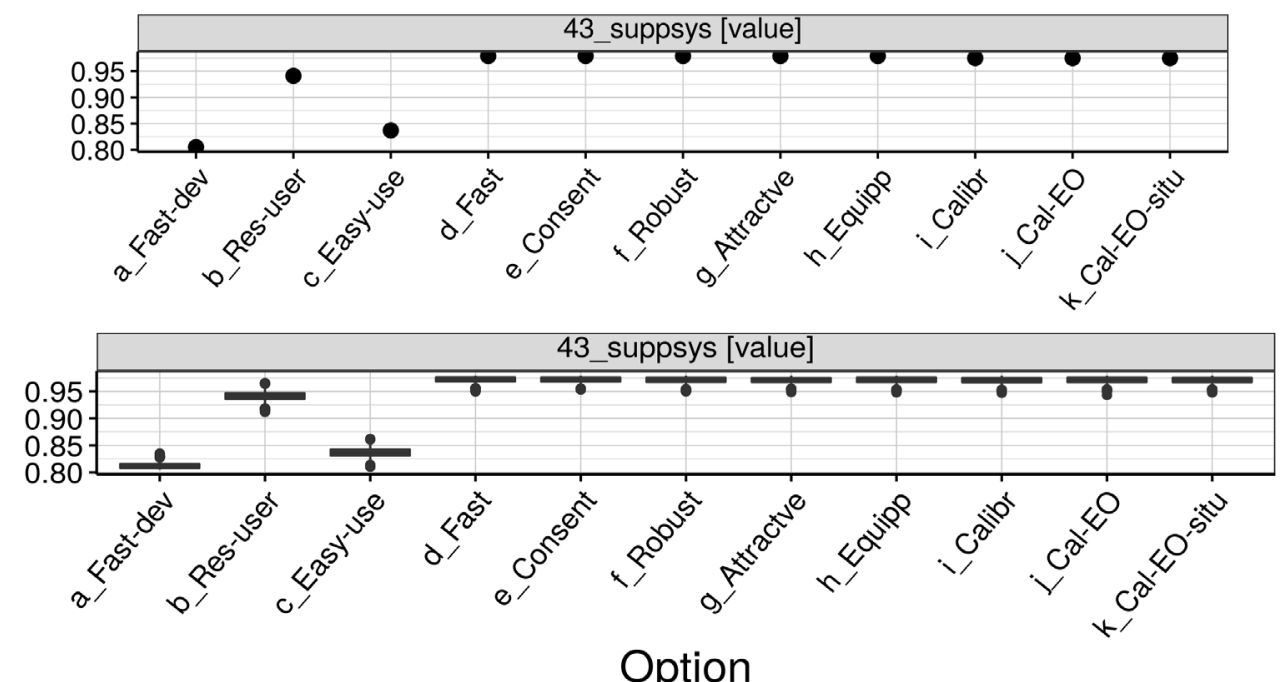

Figure SI-29: Visualization of the aggregated value of predictions of all system configurations for the attribute 43_suppsys. The plot on top represents the predictions without uncertainty, while the plot below shows the uncertainty distribution resulting from the Monte Carlo Simulation with 1'000 runs. 


\subsubsection{Summary of predictions}

We summarized the information on how the predictions were calculated for each attribute given above (sect. SI-2.4.1) in overview tables: overview for the attributes belonging to the objectives High information accuracy and clarity, and Good information access (Table SI-28), and for the attributes of the remaining objectives of Low costs, and High sustainability (Table SI-29). To be able to model the MCDA, e.g., in the ValueDecisions app, this information has to be summarized again, and uncertainty needs to be included. These input data of the predicted performance of each FANFAR system configuration, including uncertainty, are presented in Table SI-30. Furthermore, we visualized the predicted performance on each attribute with and without uncertainty in the output graphs from the ValueDecisions app (Figure SI-30).

Table SI-28: Summary raw data of how predictions were calculated for each FANFAR system configuration (left column) based on sub-attributes for the objectives High information accuracy and clarity, and Good information access. Indicated bold are the aggregated values $(v)$, ranging from [0,1], or the aggregated score for each attribute, stemming from the predictions on sub-attributes.

\begin{tabular}{|c|c|c|c|c|c|c|c|c|c|c|c|c|c|c|c|c|c|}
\hline \multirow[b]{4}{*}{ Configuration } & \multicolumn{17}{|c|}{ Objective } \\
\hline & \multicolumn{8}{|c|}{ High information accuracy and clarity } & \multicolumn{9}{|c|}{ Good information access } \\
\hline & \multicolumn{4}{|c|}{$\begin{array}{l}\text { High accuracy } \\
\text { of information }\end{array}$} & \multicolumn{4}{|c|}{$\begin{array}{l}\text { Clear flood risk } \\
\text { information }\end{array}$} & \multicolumn{4}{|c|}{$\begin{array}{l}\text { Reliable access } \\
\text { to information }\end{array}$} & \multicolumn{4}{|c|}{$\begin{array}{l}\text { Timely production, distribution, } \\
\text { and access to info }\end{array}$} & \multirow{2}{*}{$\begin{array}{l}\begin{array}{l}\text { Several } \\
\text { languages }\end{array} \\
\text { Weighted } \\
\text { sum } \\
\text { (score) }\end{array}$} \\
\hline & 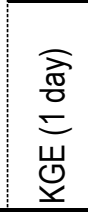 & 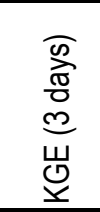 & 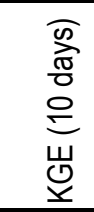 & $\begin{array}{l}\text { Aggre- } \\
\text { gated } \\
\text { value } \\
\text { (v) }\end{array}$ & $\begin{array}{l}\text { Place } \\
\text { where flood } \\
\text { risk is de- } \\
\text { fined }\end{array}$ & $\begin{array}{l}\text { Possible } \\
\text { to calcu- } \\
\text { late flood } \\
\text { risk? }\end{array}$ & $\begin{array}{l}\text { Possible } \\
\text { to apply } \\
\text { to flood } \\
\text { manage- } \\
\text { ment? }\end{array}$ & $\begin{array}{l}\text { Aggrega- } \\
\text { ted value } \\
\text { (v) }\end{array}$ & $\begin{array}{l}\text { Reliability } \\
\text { of SMS }\end{array}$ & $\begin{array}{l}\text { Reliability } \\
\text { of Email }\end{array}$ & $\begin{array}{l}\text { Reliability } \\
\text { of web } \\
\text { fanfar_eu }\end{array}$ & $\begin{array}{l}\text { Weighted } \\
\text { sum } \\
\text { (score) }\end{array}$ & $\begin{array}{l}\text { Pro- } \\
\text { duc- } \\
\text { tion } \\
\text { time } \\
\text { (hour) }\end{array}$ & $\begin{array}{l}\text { Access via } \\
\text { distribution } \\
\text { channel } \\
\text { (min) }\end{array}$ & $\begin{array}{l}\text { Ac- } \\
\text { cess } \\
\text { via H- } \\
\text { TEP } \\
\text { (min.) }\end{array}$ & $\begin{array}{l}\text { Aggregated } \\
\text { value } \\
\text { (v) }\end{array}$ & \\
\hline a_Fast-dev & 0.30 & 0.15 & -2.00 & 0.45 & Everywhere & Yes & No & 0.667 & none & none & sporadic & 0.35 & 4.00 & 2.00 & 10.00 & 0.511 & 10.10 \\
\hline b_Res-user & 0.79 & 0.49 & -1.94 & 0.65 & Everywhere & Yes & Yes & 1.000 & stable & stable & sporadic & 2.05 & 4.00 & 0.50 & 12.00 & 0.618 & 17.64 \\
\hline c_Easy-use & 0.42 & 0.24 & -1.99 & 0.48 & Everywhere & Yes & Yes & 1.000 & sporadic & sporadic & stable & 1.69 & 5.80 & 1.00 & 11.00 & 0.526 & 17.44 \\
\hline d_Fast & 0.46 & 0.26 & -1.98 & 0.50 & Everywhere & Yes & No & 0.667 & stable & stable & stable & 2.20 & 4.50 & 0.50 & 10.00 & 0.633 & 10.10 \\
\hline e_Consent & 0.86 & 0.58 & 0.40 & 0.74 & Everywhere & Yes & Yes & 1.000 & stable & stable & sporadic & 2.05 & 4.50 & 0.50 & 15.00 & 0.567 & 17.44 \\
\hline f_Robust & 0.46 & 0.26 & 0.40 & 0.54 & Everywhere & Yes & Yes & 1.000 & stable & stable & sporadic & 2.05 & 6.30 & 0.50 & 13.00 & 0.557 & 17.64 \\
\hline g_Attractve & 0.86 & 0.58 & 0.40 & 0.74 & Everywhere & Yes & Yes & 1.000 & stable & stable & sporadic & 2.05 & 5.50 & 0.50 & 15.00 & 0.544 & 17.64 \\
\hline h_Equipp & 0.86 & 0.58 & 0.40 & 0.74 & Everywhere & Yes & Yes & 1.000 & stable & stable & sporadic & 2.05 & 6.30 & 0.50 & 15.00 & 0.531 & 17.64 \\
\hline i_Calibr & 0.65 & 0.49 & 0.40 & 0.64 & Everywhere & Yes & No & 0.667 & stable & stable & sporadic & 2.05 & 6.30 & 0.50 & 12.00 & 0.571 & 16.80 \\
\hline j_Cal-EO & 0.72 & 0.58 & 0.40 & 0.68 & Everywhere & Yes & No & 0.667 & stable & stable & sporadic & 2.05 & 5.00 & 0.50 & 12.00 & 0.596 & 16.80 \\
\hline k_Cal-EO-situ & 0.93 & 0.75 & 0.40 & 0.83 & Everywhere & Yes & No & 0.667 & stable & stable & sporadic & 2.05 & 5.50 & 0.50 & 13.00 & 0.571 & 16.80 \\
\hline
\end{tabular}


Table SI-29: Summary raw data of how predictions were calculated for each FANFAR system configuration (left column) based on sub-attributes for the objectives Low costs, and High sustainability. Indicated bold are the aggregated values $(\mathrm{v})$, ranging from $[0,1]$, or the aggregated score for each attribute, stemming from the predictions on sub-attributes.

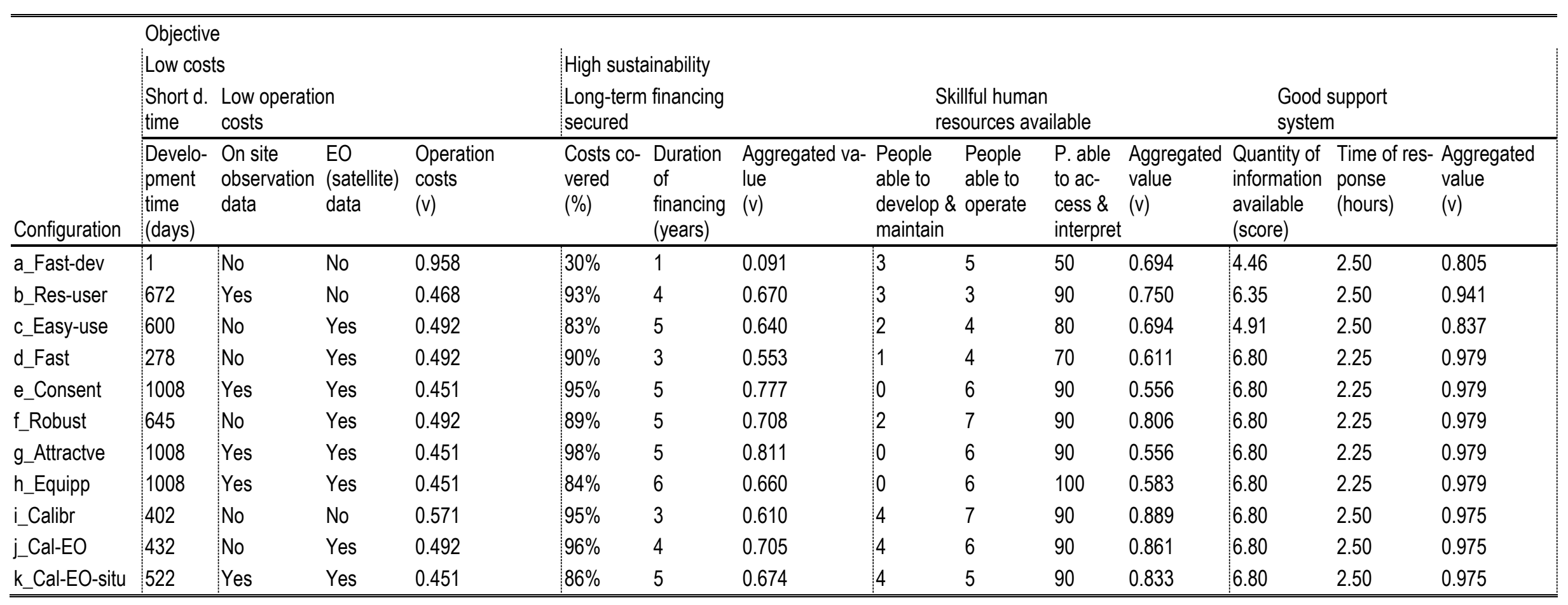


Table SI-30. Predicted performance of all 11 FANFAR system configurations (a-k, columns) on each objective, respectively attribute (first column), based on expert estimates. Prediction: predicted performance of each configuration; unc_distr: uncertainty distributions used for MCDA modeling with corresponding parameters: mean and standard deviation (SD) for normal distribution; mode, min, and max for triangular distribution (triang).

\begin{tabular}{|c|c|c|c|c|c|c|c|c|c|c|c|c|}
\hline Attribute & Parameter & $\begin{array}{l}\text { a_Fast- } \\
\text { dev }\end{array}$ & $\begin{array}{l}\text { b_Res- } \\
\text { user }\end{array}$ & $\begin{array}{l}\text { c_Easy- } \\
\text { use }\end{array}$ & d_Fast & $\begin{array}{l}\text { e_Con- } \\
\text { sent }\end{array}$ & f_Ro & $\begin{array}{l}\text { g_Attrac- } \\
\text { tve }\end{array}$ & $\begin{array}{l}\text { h_Equip } \\
\text { p }\end{array}$ & i_Calibr & j_Cal- & $\begin{array}{l}\text { k_Cal- } \\
\text { EO-situ }\end{array}$ \\
\hline \multirow{4}{*}{$\begin{array}{l}11 \_\mathrm{kge} \\
\text { value } \\
{[0: 1]}\end{array}$} & Prediction & 0.45 & 0.646 & 0.484 & 0.497 & 0.738 & 0.54 & 0.738 & 0.738 & 0.638 & 0.679 & 0.833 \\
\hline & unc_distr & normal & normal & normal & normal & normal & normal & normal & normal & normal & normal & normal \\
\hline & Mean & 0.437 & 0.554 & 0.461 & 0.471 & 0.588 & 0.485 & 0.587 & 0.588 & 0.542 & 0.564 & 0.625 \\
\hline & SD & 0.027 & 0.049 & 0.030 & 0.031 & 0.056 & 0.034 & 0.055 & 0.058 & 0.043 & 0.048 & 0.068 \\
\hline \multirow{2}{*}{$\begin{array}{l}12 \text { info } \\
\text { value } \\
{[0: 1]}\end{array}$} & Prediction & 0.667 & 1 & 1 & 0.667 & 1 & 1 & 1 & 1 & 0.667 & 0.667 & 0.667 \\
\hline & unc_distr & none & none & none & none & none & none & none & none & none & none & none \\
\hline \multirow{4}{*}{$\begin{array}{l}\text { 21_channel } \\
\text { score } \\
{[0: 2.2]}\end{array}$} & Prediction & 0.35 & 2.05 & 1.69 & 2.20 & 2.05 & 2.05 & 2.05 & 2.05 & 2.05 & 2.05 & 2.05 \\
\hline & unc_distr & normal & normal & normal & normal & normal & normal & normal & normal & normal & normal & normal \\
\hline & Mean & 0.35 & 2.05 & 1.69 & 2.2 & 2.05 & 2.05 & 2.05 & 2.05 & 2.05 & 2.05 & 2.05 \\
\hline & SD & 0.035 & 0.205 & 0.169 & 0.22 & 0.205 & 0.205 & 0.205 & 0.205 & 0.205 & 0.205 & 0.205 \\
\hline \multirow{4}{*}{$\begin{array}{l}22 \text { 2_time } \\
\text { value } \\
{[0: 1]}\end{array}$} & Prediction & 0.511 & 0.618 & 0.526 & 0.633 & 0.567 & 0.557 & 0.544 & 0.531 & 0.571 & 0.596 & 0.571 \\
\hline & unc_distr & normal & normal & normal & normal & normal & normal & normal & normal & normal & normal & normal \\
\hline & Mean & 0.499 & 0.611 & 0.528 & 0.612 & 0.579 & 0.558 & 0.558 & 0.546 & 0.564 & 0.587 & 0.571 \\
\hline & SD & 0.040 & 0.031 & 0.031 & 0.031 & 0.028 & 0.027 & 0.027 & 0.026 & 0.027 & 0.029 & 0.029 \\
\hline \multirow{3}{*}{$\begin{array}{l}\text { 23_langue } \\
\text { score } \\
{[0: 17.64]}\end{array}$} & Prediction & 10.1 & 17.64 & 17.44 & 10.1 & 17.44 & 17.64 & 17.64 & 17.64 & 16.8 & 16.8 & 16.8 \\
\hline & unc_distr & none & none & none & none & none & none & none & none & none & none & none \\
\hline & Prediction & 1 & 672 & 600 & 278 & 1008 & 645 & 1008 & 1008 & 402 & 432 & 522 \\
\hline \multirow{4}{*}{$\begin{array}{l}31 \text { 31_devlptime } \\
\text { days } \\
{[0: 1095]}\end{array}$} & unc_distr & triang & triang & triang & triang & triang & triang & triang & triang & triang & triang & triang \\
\hline & Mode & 1 & 537 & 480 & 222 & 806 & 516 & 806 & 806 & 321 & 345 & 417 \\
\hline & Min & 1.01 & 806 & 720 & 333 & 1095 & 774 & 1095 & 1095 & 482 & 518 & 626 \\
\hline & Max & 1.001 & 672 & 600 & 278 & 1008 & 645 & 1008 & 1008 & 402 & 432 & 522 \\
\hline \multirow{5}{*}{$\begin{array}{l}32 \text { costs } \\
\text { Value } \\
{[0: 1]}\end{array}$} & Prediction & 0.958 & 0.468 & 0.492 & 0.492 & 0.451 & 0.492 & 0.451 & 0.451 & 0.571 & 0.492 & 0.451 \\
\hline & unc_distr & triang & triang & triang & triang & triang & triang & triang & triang & triang & triang & triang \\
\hline & Mode & 0.933 & 0.441 & 0.472 & 0.472 & 0.416 & 0.472 & 0.416 & 0.416 & 0.497 & 0.472 & 0.416 \\
\hline & Min & 0.983 & 0.495 & 0.587 & 0.587 & 0.486 & 0.587 & 0.486 & 0.486 & 0.662 & 0.587 & 0.486 \\
\hline & Max & 0.958 & 0.468 & 0.492 & 0.492 & 0.451 & 0.492 & 0.451 & 0.451 & 0.571 & 0.492 & 0.451 \\
\hline \multirow{4}{*}{$\begin{array}{l}\text { 41_finance } \\
\text { value } \\
{[0: 1]}\end{array}$} & Prediction & 0.091 & 0.67 & 0.64 & 0.553 & 0.777 & 0.708 & 0.811 & 0.66 & 0.61 & 0.705 & 0.674 \\
\hline & unc_distr & normal & normal & normal & normal & normal & normal & normal & normal & normal & normal & normal \\
\hline & Mean & 0.073 & 0.632 & 0.606 & 0.534 & 0.731 & 0.676 & 0.754 & 0.653 & 0.572 & 0.654 & 0.641 \\
\hline & SD & 0.01025 & 0.036 & 0.038 & 0.037 & 0.033 & 0.038 & 0.028 & 0.034 & 0.032 & 0.032 & 0.035 \\
\hline \multirow{4}{*}{$\begin{array}{l}\text { 42_experts } \\
\text { value } \\
{[0: 1]}\end{array}$} & Prediction & 0.694 & 0.75 & 0.694 & 0.611 & 0.556 & 0.806 & 0.556 & 0.583 & 0.889 & 0.861 & 0.833 \\
\hline & unc_distr & normal & normal & normal & normal & normal & normal & normal & normal & normal & normal & normal \\
\hline & Mean & 0.69 & 0.72 & 0.685 & 0.591 & 0.531 & 0.78 & 0.532 & 0.546 & 0.862 & 0.836 & 0.81 \\
\hline & SD & 0.025 & 0.029 & 0.032 & 0.048 & 0.028 & 0.034 & 0.030 & 0.029 & 0.029 & 0.031 & 0.025 \\
\hline \multirow{4}{*}{$\begin{array}{l}\text { 43_suppsys } \\
\text { value } \\
{[0: 1]}\end{array}$} & Prediction & 0.805 & 0.941 & 0.837 & 0.979 & 0.979 & 0.979 & 0.979 & 0.979 & 0.975 & 0.975 & 0.975 \\
\hline & unc_distr & normal & normal & normal & normal & normal & normal & normal & normal & normal & normal & normal \\
\hline & Mean & 0.806 & 0.941 & 0.837 & 0.977 & 0.977 & 0.976 & 0.976 & 0.976 & 0.975 & 0.975 & 0.975 \\
\hline & SD & 0.009 & 0.008 & 0.008 & 0.009 & 0.009 & 0.009 & 0.009 & 0.009 & 0.008 & 0.008 & 0.009 \\
\hline
\end{tabular}



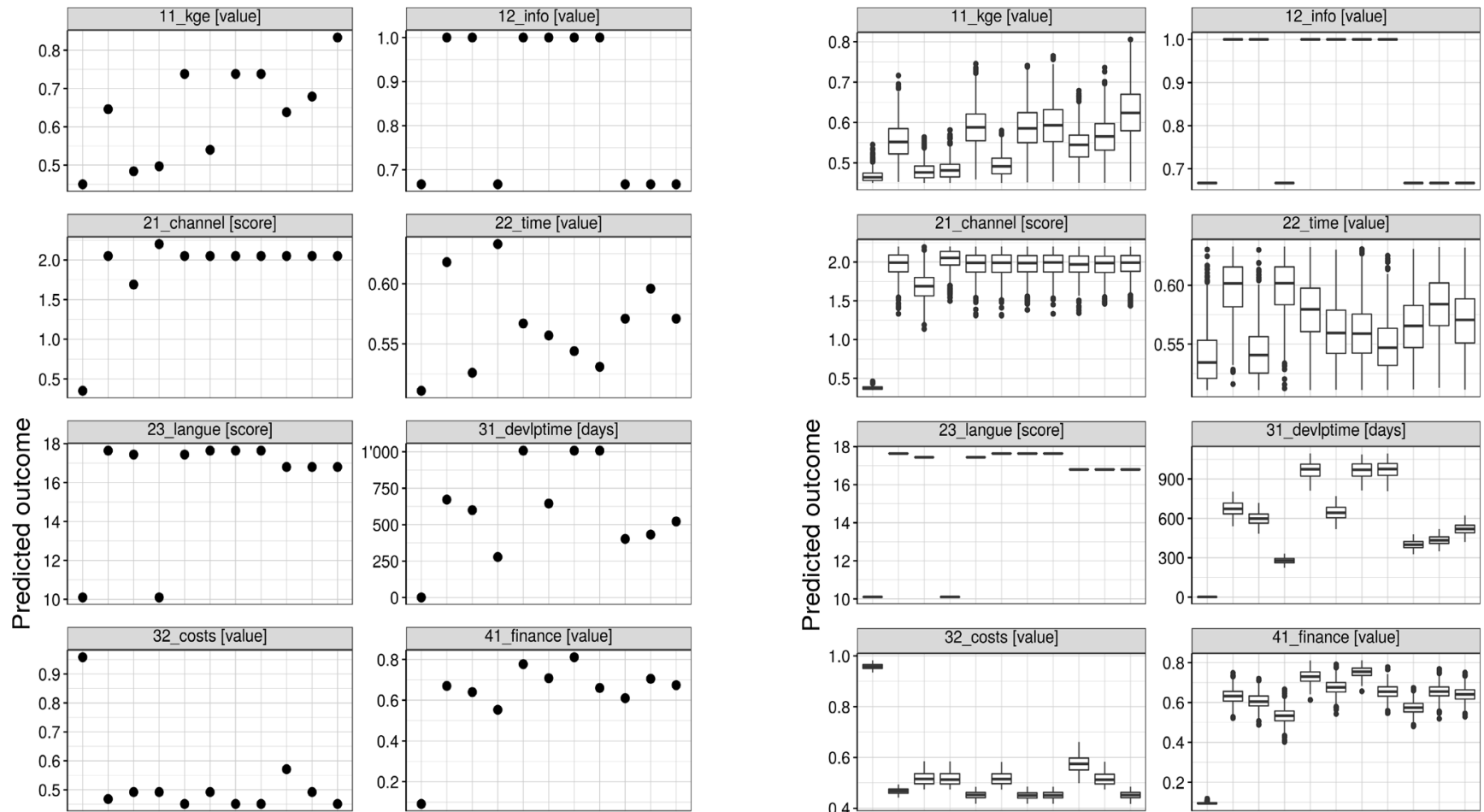

ถุ
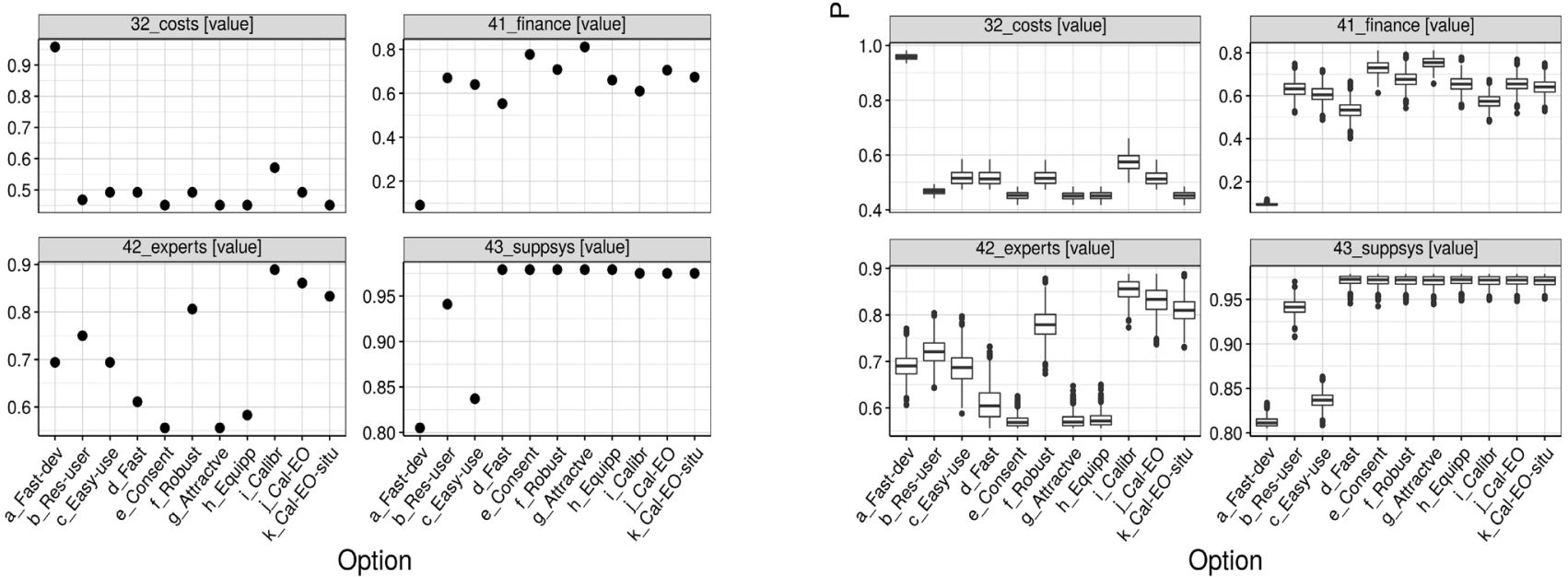

Figure SI-30. Predicted performance (y-axis) of each FANFAR system configuration (x-axis) on each attribute (boxes), according to the expert elicitation. Left panel: without uncertainty, right panel: with uncertainty). 


\subsection{Marginal value functions}
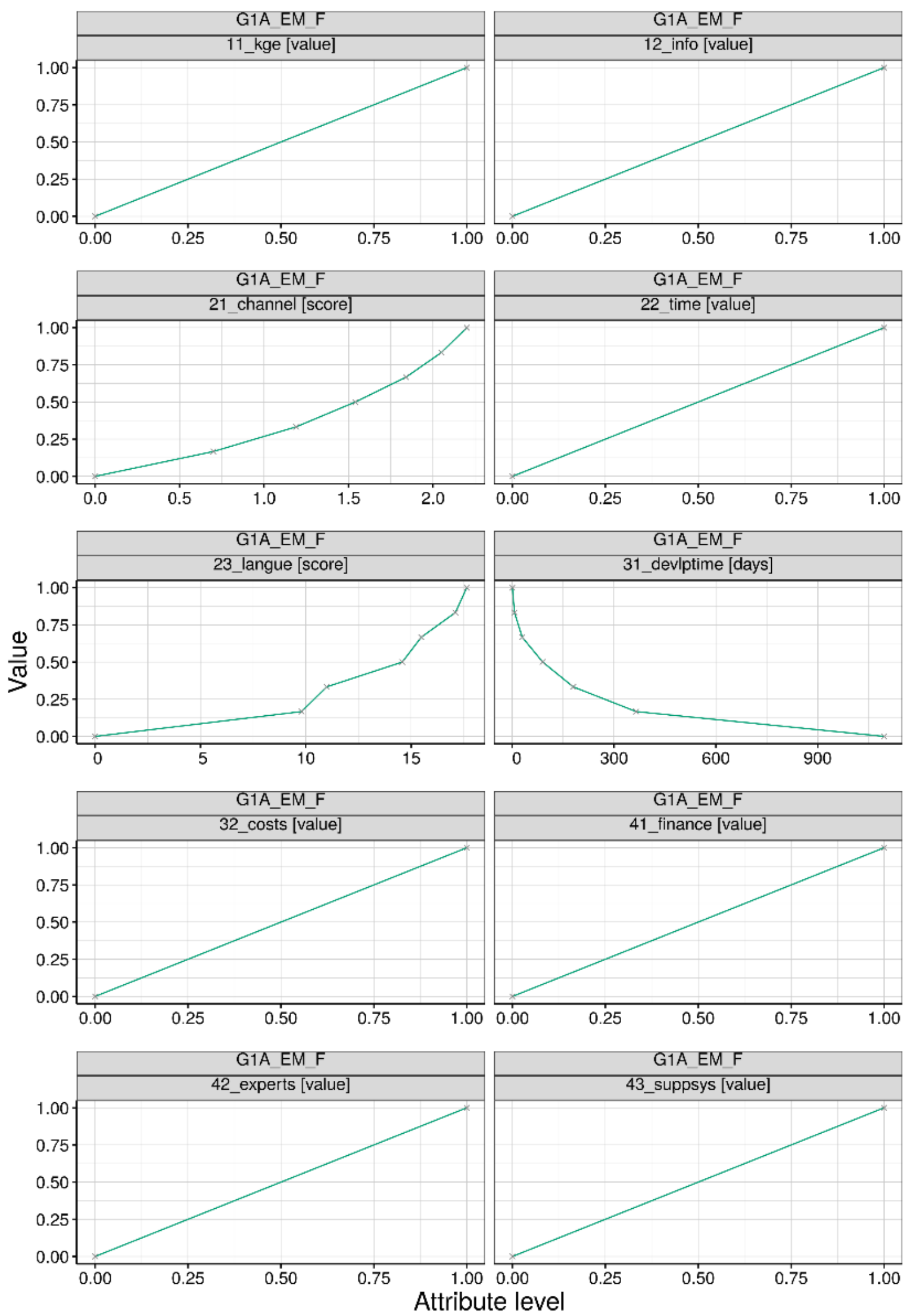

Figure SI-31. Marginal value functions used to transform predicted outcome of attributes into values. The example shows stakeholder group G1A_EM_F, but the marginal value functions were the same for all stakeholders in our case (see main text). 


\subsection{Weights}

Over all groups, highest weights were assigned to the two objectives within the branch 1 . High information accuracy and clarity: High accuracy of information (11_accur_info; median over all groups $=0.150 ; 0.25$ lower quartile $=0.134 ; 0.75$ upper quartile $=0.169$ ), and Clear flood risk information (12_clear_info; median=0.152; lower=0.134; upper=0.161; Figure SI-32). For 11_accur_info, there were two outliers: G1A_EM_F assigned an exceptionally low weight of 0.065, and G2A_HY_F assigned an exceptionally high weight of 0.251 . Generally, slightly lower weights were assigned to Timely production, distribution and access to info (22_timely_info; median $=0.138$; lower $=0.102$; upper $=0.150)$, closely followed by Reliable access to information (21_reliable_info; median $=0.116$; lower $=0.094$; upper $=0.142$ ), and Skillful human resources available (42_human_resour; median $=0.106$; lower $=0.102$; upper $=0.126$ ). Group G1A_EM_F, with an exceptionally high weight of 0.25 , formed the outlier in 42 _human_resour.

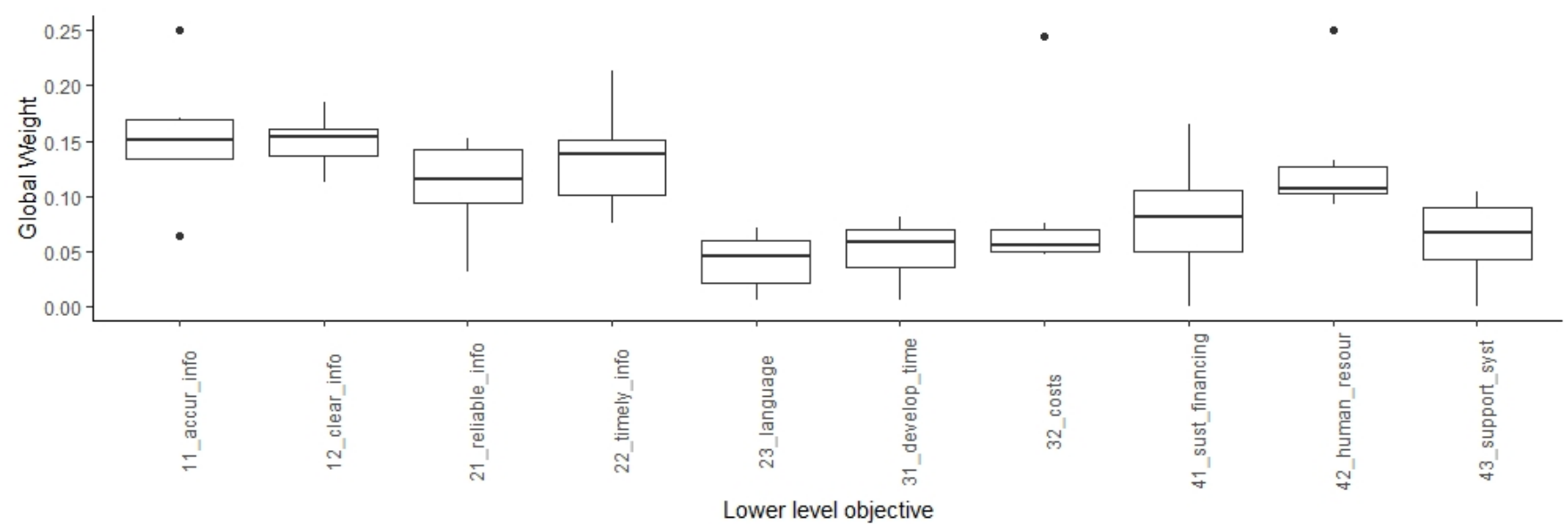

Figure SI-32. Global weight of each lower level objective over all stakeholder groups. The boxplot shows the $0.25,0.5$, and 0.75 quartiles of weights for each lower level objective. The whiskers extend to maximum and minimum points within 1.5 times the interquartile range. Points outside whiskers are outliers. Uncertainty of preference statements within groups are not represented.

Intermediate weights were assigned to Long-term financing secured (41_sust_financing; median $=0.081 ;$ lower $=0.050 ;$ upper $=0.105$ ), and Good support system (43_support_syst; median $=0,067$; lower $=0.042$; upper $=0.090$ ). Over all groups, low weights were assigned to Several languages (23_language; median $=0.045 ;$ lower $=0.021 ;$ upper $=0.060)$, Short development time (31_develop_time; median $=0.059$; lower $=0.035$; upper $=0.070)$, and Low acquisition and operation costs $\left(32 \_\right.$costs; (median $=0.056$; lower $=0.036$; upper $=0.070)$. G1A_EM_F was again an outlier for $32 \_$costs with an exceptionally high weight of 0.244 .

The difference between groups in the assigned importance of objectives was notably larger for some objectives, e.g., 21_reliable_info, 22_timely_info, 41_sust_finance, and 43_support_syst (large interquartile ranges in Figure SI-32). Moreover, the strongly differing preferences of group G1A_EM_F is visualized by outliers in the boxplot of the higher level objectives, namely for 11_accur_info, 32_costs, and 42_human_resour (Figure SI-33). 


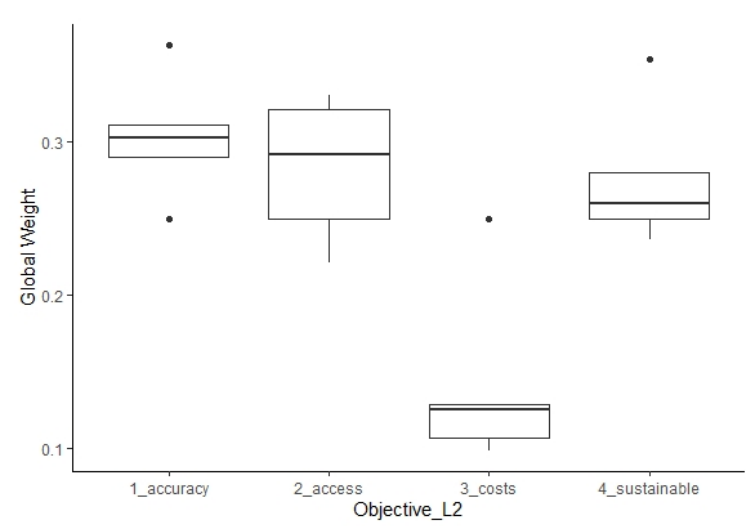

Figure SI-33. Global weight assigned to each higher level objective over all stakeholder groups. The boxplot shows the $0.25,0.5$, and 0.75 quartiles of weights for each lower level objective. The whiskers extend to the maximum and minimum points within 1.5 times the interquartile range. Points outside the whiskers are outliers. Uncertainty of preference statements within individual groups are not represented.

Table SI-31. Elicited weights and ranges for each stakeholder group (G1A_EM_F to G5A_AGRHYMET), elicited with the Swing or Simos' revised card procedure (see main text). We indicate the average local and global weights for each sub-objective, and the minimum and maximum range of the local and global weights. Note: the local weight is that weight assigned to a sub-objective within one branch of the hierarchy, which sums up to one. For instance, the local weights of sub-objective "1.1 High accuracy of information" and "1.2 Clear flood risk information" sum up to 1 for the higher level objective "1 High information accuracy and clarity". Over all ten sub-objectives of the hierarchy, the global weights sum up to 1 . We also indicate the minimum and maximum, respectively, of the weight ranges as elicited in the stakeholder workshops, and the deviation from the average in percent. Yellow highlights: deviation $>0.02$, which was tested in local sensitivity analyses. Green highlights: deviation $>0.02$, but not tested in sensitivity analyses because objective was not sensitive to weight changes (see Methods).

\begin{tabular}{|c|c|c|c|c|c|c|}
\hline \multicolumn{7}{|l|}{$\overline{\text { G1A_EM_F (Swing) }}$} \\
\hline Objective & $\begin{array}{l}\text { Average } \\
\text { local } \\
\text { weight (w) }\end{array}$ & $\begin{array}{l}\text { Local weight } \\
\text { range min } \\
\text { (wmin) }\end{array}$ & $\begin{array}{l}\text { Local weight } \\
\text { range max } \\
\text { (wmax) }\end{array}$ & $\begin{array}{l}\text { Average glo- } \\
\text { bal weight } \\
\text { (W)- }\end{array}$ & $\begin{array}{l}\text { Global weight } \\
\text { range min } \\
\text { (Wmin) }\end{array}$ & $\begin{array}{l}\text { Global weight } \\
\text { range max } \\
\text { (Wmax) }\end{array}$ \\
\hline 1.1 High accuracy of information & 0.259 & 0.167 & 0.333 & 0.065 & $0.042(-0.023)$ & $0.083(+0.019)$ \\
\hline 1.2 Clear flood risk information & 0.741 & 0.667 & 0.833 & 0.185 & $0.167(-0.019)$ & $0.208(+0.023)$ \\
\hline 2.1 Reliable access to information & 0.128 & 0.087 & 0.167 & 0.032 & $0.022(-0.010)$ & $0.042(+0.010)$ \\
\hline 2.2 Timely production, distribution, access to info & 0.851 & 0.800 & 0.909 & 0.213 & $0.200(-0.013)$ & $0.227(+0.015)$ \\
\hline 2.3 Several languages & 0.021 & 0.000 & 0.043 & 0.005 & $0.000(-0.005)$ & $0.011(+0.006)$ \\
\hline 3.1 Short development time & 0.024 & 0.000 & 0.048 & 0.006 & $0.000(-0.006)$ & $0.012(+0.006)$ \\
\hline 3.2 Low acquisition and operation costs & 0.976 & 0.952 & 1.000 & 0.244 & $0.238(-0.006)$ & $0.250(+0.006)$ \\
\hline 4.1 Long-term financing secured & 0.000 & 0.000 & 0.000 & 0.000 & $0.000(-0)$ & $0.000(+0)$ \\
\hline 4.2 Skillful human resources available & 1.000 & 1.000 & 1.000 & 0.250 & $0.250(-0)$ & $0.250(+0)$ \\
\hline 4.3 Good support system & 0.000 & 0.000 & 0.000 & 0.000 & $0.000(-0)$ & $0.000(+0)$ \\
\hline
\end{tabular}

G2A_HY_F (Swing)

\begin{tabular}{lllllll}
\hline Objective & $\begin{array}{l}\text { Average } \\
\text { local } \\
\text { weight }(\mathrm{w})\end{array}$ & $\begin{array}{l}\text { Local weight } \\
\text { range min } \\
\text { (wmin) }\end{array}$ & $\begin{array}{l}\text { Local weight Average glo- Global weight } \\
\text { range max } \\
\text { (wmax) }\end{array}$ & $\begin{array}{l}\text { Global weight } \\
\text { bal wht }\end{array}$ & $\begin{array}{l}\text { lange min } \\
\text { (Wmin) }\end{array}$ & $\begin{array}{l}\text { lange max } \\
\text { (Wmax) }\end{array}$ \\
\hline 1.1 High accuracy of information & 0.690 & 0.690 & 0.690 & 0.251 & $0.238(-0.013)$ & $0.265(+0.014)$ \\
1.2 Clear flood risk information & 0.310 & 0.310 & 0.310 & 0.113 & $0.107(-0.006)$ & $0.119(+0.007)$ \\
2.1 Reliable access to information & 0.400 & 0.385 & 0.417 & 0.109 & $0.096(-0.013)$ & $0.123(+0.014)$ \\
2.2 Timely production, distribution, access to info & 0.340 & 0.313 & 0.365 & 0.093 & $0.078(-0.015)$ & $0.108(+0.016)$ \\
2.3 Several languages & 0.260 & 0.250 & 0.271 & 0.071 & $0.063(-0.008)$ & $0.080(+0.009)$ \\
3.1 Short development time & 0.556 & 0.556 & 0.556 & 0.071 & $0.060(-0.011)$ & $0.082(+0.012)$ \\
3.2 Low acquisition and operation costs & 0.444 & 0.444 & 0.444 & 0.057 & $0.048(-0.009)$ & $0.066(+0.009)$ \\
4.1 Long-term financing secured & 0.449 & 0.435 & 0.465 & 0.106 & $0.093(-0.013)$ & $0.121(+0.014)$ \\
4.2 Skillful human resources available & 0.393 & 0.378 & 0.409 & 0.093 & $0.081(-0.012)$ & $0.106(+0.013)$ \\
4.3 Good support system & 0.157 & 0.136 & 0.178 & 0.037 & $0.029(-0.008)$ & $0.046(+0.009)$ \\
\hline
\end{tabular}


G2B_HY_F (Swing)

\begin{tabular}{|c|c|c|c|c|c|c|}
\hline Objective & $\begin{array}{l}\text { Average } \\
\text { local } \\
\text { weight }(w)\end{array}$ & $\begin{array}{l}\text { Local weight } \\
\text { range min } \\
\text { (wmin) }\end{array}$ & $\begin{array}{l}\text { Local weight } \\
\text { range max } \\
\text { (wmax) }\end{array}$ & $\begin{array}{l}\text { Average glo- } \\
\text { bal weight } \\
(\mathrm{W})\end{array}$ & $\begin{array}{l}\text { Global weight } \\
\text { range min } \\
\text { (Wmin) }\end{array}$ & $\begin{array}{l}\text { Global weight } \\
\text { range max } \\
\text { (Wmax) }\end{array}$ \\
\hline 1.1 High accuracy of information & 0.459 & 0.429 & 0.487 & 0.138 & $0.111(-0.027)$ & $0.168(+0.030)$ \\
\hline 1.2 Clear flood risk information & 0.541 & 0.513 & 0.571 & 0.163 & $0.133(-0.030)$ & $0.197(+0.035)$ \\
\hline 2.1 Reliable access to information & 0.400 & 0.385 & 0.417 & 0.088 & $0.067(-0.021)$ & $0.112(+0.023)$ \\
\hline 2.2 Timely production, distribution, access to info & 0.340 & 0.313 & 0.365 & 0.075 & $0.055(-0.020)$ & $0.098(+0.023)$ \\
\hline 2.3 Several languages & 0.260 & 0.250 & 0.271 & 0.058 & $0.044(-0.014)$ & $0.073(+0.015)$ \\
\hline 3.1 Short development time & 0.556 & 0.556 & 0.556 & 0.069 & $0.056(-0.013)$ & $0.084(+0.015)$ \\
\hline 3.2 Low acquisition and operation costs & 0.444 & 0.444 & 0.444 & 0.055 & $0.044(-0.011)$ & $0.067(+0.012)$ \\
\hline 4.1 Long-term financing secured & 0.465 & 0.455 & 0.476 & 0.165 & $0.147(-0.018)$ & $0.187(+0.022)$ \\
\hline 4.2 Skillful human resources available & 0.372 & 0.364 & 0.381 & 0.132 & $0.117(-0.014)$ & $0.149(+0.018)$ \\
\hline 4.3 Good support system & 0.163 & 0.143 & 0.182 & 0.058 & $0.046(-0.012)$ & $0.071(+0.014)$ \\
\hline
\end{tabular}

G3A_HY_E (Simos' card)

\begin{tabular}{lllll}
\hline Objective & rank & $\begin{array}{l}\text { Focus global } \\
\text { weight }(\mathrm{W}) \text { [Z=10] }\end{array}$ & $\begin{array}{l}\text { Global weight range } \\
\min (\text { Wmin) }\end{array}$ & $\begin{array}{l}\text { Global weight range } \\
\max (\text { Wmax })\end{array}$ \\
\hline 1.1 High accuracy of information & 1 & 0.171 & $0.159(-0.013)$ & $0.178(+0.007)$ \\
1.2 Clear flood risk information & 5 & 0.133 & $0.127(-0.006)$ & $0.136(+0.003)$ \\
2.1 Reliable access to information & 3 & 0.152 & $0.143(-0.009)$ & $0.157(+0.005)$ \\
2.2 Timely production, distribution, access to info & 3 & 0.152 & $0.143(-0.009)$ & $0.157(+0.005)$ \\
2.3 Several languages & 17 & 0.017 & $0.009(-0.008)$ & $0.032(+0.015)$ \\
3.1 Short development time & 15.5 & 0.032 & $0.025(-0.007)$ & $0.044(+0.012)$ \\
3.2 Low acquisition and operation costs & 11 & 0.075 & $0.072(-0.002)$ & $0.079(+0.004)$ \\
4.1 Long-term financing secured & 12.5 & 0.060 & $0.057(-0.004)$ & $0.067(+0.007)$ \\
4.2 Skillful human resources available & 8 & 0.104 & $0.103(-0.001)$ & $0.104(+0.0)$ \\
4.3 Good support system & 8 & 0.104 & $0.103(-0.001)$ & $0.104(+0.0)$ \\
\hline Max/Sum & 17 & 1 & - & - \\
\hline
\end{tabular}

G4A_EM_E (Simos' card)

\begin{tabular}{|c|c|c|c|c|}
\hline Objective & rank & $\begin{array}{l}\text { Focus global } \\
\text { weight }(W)[Z=3.33]\end{array}$ & $\begin{array}{l}\text { Global weight range } \\
\min (\text { Wmin) }\end{array}$ & $\begin{array}{l}\text { Global weight range } \\
\max (W \max )\end{array}$ \\
\hline 1.1 High accuracy of information & 4.5 & 0.133 & $0.126(-0.007)$ & $0.148(+0.0)$ \\
\hline 1.2 Clear flood risk information & 1 & 0.157 & $0.122(-0.012)$ & $0.157(+0.0)$ \\
\hline 2.1 Reliable access to information & 6 & 0.123 & $0.118(-0.005)$ & $0.135(+0.0)$ \\
\hline 2.2 Timely production, distribution, access to info & 2.5 & 0.147 & $0.135(-0.010)$ & $0.147(+0.0)$ \\
\hline 2.3 Several languages & 15 & 0.061 & $0.044(-0.0)$ & $0.069(+0.008)$ \\
\hline 3.1 Short development time & 12 & 0.081 & $0.054(-0.0)$ & $0.085(+0.004)$ \\
\hline 3.2 Low acquisition and operation costs & 17 & 0.047 & $0.047(-0.0)$ & $0.083(+0.011)$ \\
\hline 4.1 Long-term financing secured & 17 & 0.047 & $0.047(-0.0)$ & $0.073(+0.011)$ \\
\hline 4.2 Skillful human resources available & 8 & 0.109 & $0.103(-0.002)$ & $0.109(+0.0)$ \\
\hline 4.3 Good support system & 10 & 0.095 & $0.095(-0.0)$ & $0.103(+0.001)$ \\
\hline Max/Sum & 17 & 1 & - & - \\
\hline
\end{tabular}

G5A_AGRHYMET (Simos' card)

\begin{tabular}{lllll}
\hline Objective & rank & $\begin{array}{l}\text { Focus global } \\
\text { weight (W) [Z=5] }\end{array}$ & $\begin{array}{l}\text { Global weight range } \\
\min (\text { Wmin) }\end{array}$ & $\begin{array}{l}\text { Global weight range } \\
\max (\text { Wmax })\end{array}$ \\
\hline 1.1 High accuracy of information & 1 & 0.162 & $0.151(-0.012)$ & $0.162(+0.0)$ \\
1.2 Clear flood risk information & 3 & 0.149 & $0.140(-0.009)$ & $0.149(+0.0)$ \\
2.1 Reliable access to information & 3 & 0.149 & $0.140(-0.009)$ & $0.149(+0.0)$ \\
2.2 Timely production, distribution, access to info & 6 & 0.129 & $0.124(-0.005)$ & $0.129(+0.0)$ \\
2.3 Several languages & 20.5 & 0.032 & $0.032(-0.0)$ & $0.045(+0.013)$ \\
3.1 Short development time & 18 & 0.049 & $0.049(-0.0)$ & $0.059(+0.010)$ \\
3.2 Low acquisition and operation costs & 18 & 0.049 & $0.049(-0.0)$ & $0.059(+0.010)$ \\
4.1 Long-term financing secured & 10 & 0.102 & $0.102(-0.0)$ & $0.102(+0.0)$ \\
4.2 Skillful human resources available & 10 & 0.102 & $0.102(-0.0)$ & $0.102(+0.0)$ \\
4.3 Good support system & 14 & 0.076 & $0.076(-0.0)$ & $0.080(+0.005)$ \\
\hline Max/Sum & 20.5 & 0.999 & - & - \\
\hline
\end{tabular}




\subsection{MCDA results: values and ranks}

Table SI-32. Total aggregated value and rank of all 11 FANFAR system configurations (first column) for each stakeholder group (G1A_EM_F, G2A_HY_F, etc.), without uncertainty of predictions. The higher the value, and the lower the rank, the better the configuration achieves the objectives, given the expert predictions and the stakeholders' preferences. A total value of $v=1$ indicates that for this configuration (and stakeholder group), all objectives were able to achieve the best level (given the available system configurations). In contrast, $v=0$ indicates that only the worst level of all objectives was achieved by this configuration and this stakeholder group. Rank 1 is the best rank, i.e., the best performing configuration, rank 2 the second best, etc., and rank 11 is the worst performing configuration.

\begin{tabular}{llllllllllllll}
\hline \multirow{2}{*}{$\begin{array}{l}\text { System Configu- } \\
\text { ration }\end{array}$} & \multicolumn{2}{l}{ G1A_EM_F } & \multicolumn{3}{c}{ G2A_HY_F } & \multicolumn{3}{c}{ G2B_HY_F } & \multicolumn{2}{c}{ G3A_HY_E } & \multicolumn{2}{c}{ G4A_EM_E } & \multicolumn{3}{c}{ G5A_AGRHYMET } \\
& value & rank & value & rank & value & rank & value & rank & value & rank & value & rank \\
\hline a_Fast-dev & 0.644 & 5 & 0.398 & 11 & 0.400 & 11 & 0.433 & 11 & 0.457 & 11 & 0.398 & 11 \\
b_Res-user & 0.671 & 1 & 0.653 & 2 & 0.674 & 1 & 0.704 & 1 & 0.671 & 1 & 0.694 & 1 \\
C_Easy-use & 0.625 & 7 & 0.569 & 9 & 0.609 & 9 & 0.609 & 10 & 0.594 & 9 & 0.605 & 10 \\
d_Fast & 0.590 & 11 & 0.545 & 10 & 0.565 & 10 & 0.641 & 9 & 0.588 & 10 & 0.616 & 9 \\
e_Consent & 0.608 & 8 & 0.617 & 7 & 0.629 & 7 & 0.676 & 6 & 0.609 & 7 & 0.660 & 7 \\
f_Robust & 0.669 & 2 & 0.631 & 5 & 0.671 & 2 & 0.686 & 4 & 0.659 & 2 & 0.680 & 3 \\
g_Attractve & 0.603 & 10 & 0.620 & 6 & 0.634 & 6 & 0.674 & 7 & 0.609 & 6 & 0.661 & 6 \\
h_Equipp & 0.607 & 9 & 0.608 & 8 & 0.615 & 8 & 0.667 & 8 & 0.604 & 8 & 0.648 & 8 \\
i_Calibr & 0.668 & 3 & 0.633 & 4 & 0.645 & 5 & 0.682 & 5 & 0.646 & 5 & 0.664 & 5 \\
j_Cal-EO & 0.648 & 4 & 0.647 & 3 & 0.658 & 4 & 0.689 & 3 & 0.651 & 4 & 0.676 & 4 \\
k_Cal-EO-situ & 0.632 & 6 & 0.665 & 1 & 0.660 & 3 & 0.698 & 2 & 0.654 & 3 & 0.684 & 2 \\
\hline \hline
\end{tabular}

Table SI-33. Results of the MCDA without uncertainty. For each FANFAR system configuration (e.g., a Fast-dev, etc.) and each stakeholder group (e.g., G1A_EM_F, etc.). We show the partial values of each lower level objective (partial), and the total value aggregated over all objectives (aggregated). Partial values were calculated with objectives' weights according to the aggregation model (attribute value $^{0.2} \times$ objective weight), and the total aggregated according to the aggregation model (sum of partial values ${ }^{1 / 0.2}$ ).

\begin{tabular}{|c|c|c|c|c|c|c|c|c|c|c|c|c|c|}
\hline \multirow[b]{2}{*}{ 高 } & \multirow[b]{2}{*}{ objective } & \multicolumn{2}{|c|}{ G1A_EM_F } & \multicolumn{2}{|c|}{ G2A_HY_F } & \multicolumn{2}{|c|}{ G2B_HY_F } & \multicolumn{2}{|c|}{ G3A_HY_E } & \multicolumn{2}{|c|}{ G4A_EM_E } & \multicolumn{2}{|c|}{ G5A_AGRHYMET } \\
\hline & & 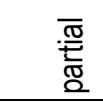 & 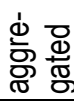 & 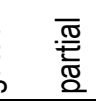 & 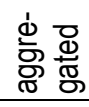 & 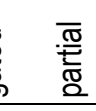 & 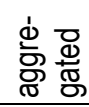 & 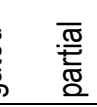 & 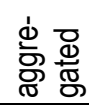 & 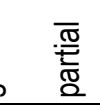 & 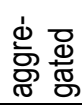 & & 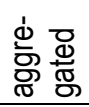 \\
\hline \multirow{10}{*}{ 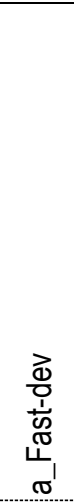 } & 11_accur_info & 0.055 & \multirow{10}{*}{ 㠻 } & 0.214 & \multirow{10}{*}{ ণ্ల్ల } & 0.118 & \multicolumn{2}{|r|}{0.146} & \multicolumn{2}{|r|}{0.113} & \multicolumn{2}{|r|}{0.138} & \\
\hline & 12_clear_info & 0.171 & & 0.104 & & 0.150 & & 0.123 & \multicolumn{2}{|r|}{0.145} & \multicolumn{2}{|r|}{0.137} & \\
\hline & 21_reliable_info & 0.019 & & 0.066 & & 0.054 & & 0.093 & \multicolumn{2}{|r|}{0.075} & \multicolumn{2}{|r|}{0.091} & \\
\hline & 22_timely_info & 0.186 & & 0.081 & & 0.066 & & 0.133 & \multicolumn{2}{|r|}{0.129} & \multicolumn{2}{|r|}{0.113} & \\
\hline & 23_language & 0.004 & & 0.052 & & 0.042 & & 0.012 & \multicolumn{2}{|r|}{0.045} & \multicolumn{2}{|r|}{0.023} & \\
\hline & 31_develop_time & 0.006 & & 0.071 & & 0.069 & & 0.032 & \multicolumn{2}{|r|}{0.081} & \multicolumn{2}{|r|}{0.049} & \\
\hline & 32_costs & 0.242 & & 0.057 & & 0.055 & & 0.074 & \multicolumn{2}{|r|}{0.047} & \multicolumn{2}{|r|}{0.049} & \\
\hline & 41_sust_financing & 0.000 & & 0.066 & & 0.102 & & 0.037 & \multicolumn{2}{|r|}{0.029} & \multicolumn{2}{|r|}{0.063} & \\
\hline & 42_human_resour & 0.232 & & 0.086 & & 0.123 & 8 & 0.097 & \multirow{2}{*}{$\stackrel{\mathscr{m}}{\stackrel{0}{0}}$} & 0.101 & \multirow{2}{*}{ 促 } & 0.095 & \& \\
\hline & 43_support_syst & 0.000 & & 0.035 & & 0.056 & f. & 0.100 & & 0.091 & & 0.073 & m. \\
\hline \multirow{10}{*}{ 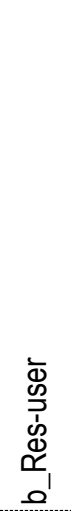 } & 11_accur_info & 0.060 & & 0.230 & & 0.126 & & 0.157 & & 0.122 & & 0.148 & \\
\hline & 12_clear_info & 0.185 & & 0.113 & & 0.163 & & 0.133 & & 0.157 & & 0.149 & \\
\hline & 21_reliable_info & 0.031 & & 0.105 & & 0.085 & & 0.147 & & 0.119 & & 0.144 & \\
\hline & 22_timely_info & 0.193 & & 0.084 & & 0.068 & & 0.138 & & 0.134 & & 0.117 & \\
\hline & 23_language & 0.005 & & 0.071 & & 0.058 & & 0.017 & & 0.061 & & 0.032 & \\
\hline & 31_develop_time & 0.004 & & 0.045 & & 0.043 & & 0.020 & & 0.051 & & 0.031 & \\
\hline & 32_costs & 0.210 & & 0.049 & & 0.047 & & 0.064 & & 0.040 & & 0.042 & \\
\hline & 41_sust_financing & 0.000 & & 0.098 & & 0.152 & & 0.055 & & 0.043 & & 0.094 & \\
\hline & 42_human_resour & 0.236 & $\Sigma$ & 0.088 & ח & 0.125 & N & 0.098 & \& & 0.103 & $\Sigma$ & 0.096 & \\
\hline & 43_support_syst & 0.000 & $\stackrel{6}{\circ}$ & 0.037 & ڤं & 0.057 & $\stackrel{0}{0}$ & 0.103 & $\tilde{\sigma}$ & 0.094 & ๕ִ & 0.075 & 0 \\
\hline
\end{tabular}




\begin{tabular}{|c|c|c|c|c|c|c|c|c|c|c|c|c|c|}
\hline \multirow[b]{2}{*}{ 동 } & \multirow[b]{2}{*}{ objective } & \multicolumn{2}{|c|}{ G1A_EM_F } & \multicolumn{2}{|c|}{ G2A_HY_F } & \multicolumn{2}{|c|}{ G2B_HY_F } & \multicolumn{2}{|c|}{ G3A_HY_E } & \multicolumn{2}{|c|}{ G4A_EM_E } & \multicolumn{2}{|c|}{ G5A_AGRHYMET } \\
\hline & & 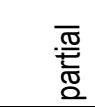 & 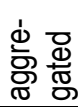 & 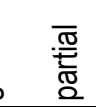 & 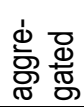 & 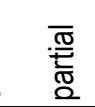 & 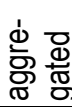 & 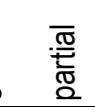 & 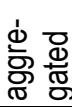 & 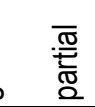 & 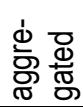 & 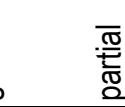 & 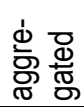 \\
\hline \multirow{10}{*}{ 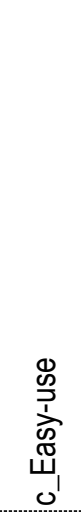 } & 11_accur_info & 0.056 & \multirow{10}{*}{ ญ్రి } & 0.217 & \multirow{10}{*}{$\begin{array}{l}8 \\
0 \\
0 \\
0\end{array}$} & 0.119 & \multicolumn{2}{|r|}{0.148} & \multicolumn{2}{|r|}{0.115} & \multicolumn{2}{|r|}{0.140} & \\
\hline & 12_clear_info & 0.185 & & 0.113 & & 0.163 & & 0.133 & \multicolumn{2}{|r|}{0.157} & \multicolumn{2}{|r|}{0.149} & \\
\hline & 21_reliable_info & 0.029 & & 0.098 & & 0.079 & & 0.136 & \multicolumn{2}{|r|}{0.110} & \multicolumn{2}{|r|}{0.134} & \\
\hline & 22_timely_info & 0.187 & & 0.082 & & 0.066 & & 0.134 & \multicolumn{2}{|r|}{0.129} & \multicolumn{2}{|r|}{0.113} & \\
\hline & 23_language & 0.005 & & 0.070 & & 0.057 & & 0.017 & \multicolumn{2}{|r|}{0.060} & & 0.032 & \\
\hline & 31_develop_time & 0.004 & & 0.046 & & 0.045 & & 0.021 & & 0.052 & & 0.032 & \\
\hline & 32_costs & 0.212 & & 0.049 & & 0.048 & & 0.065 & & 0.041 & & 0.043 & \\
\hline & 41_sust_financing & 0.000 & & 0.097 & & 0.151 & & 0.055 & & 0.043 & & 0.093 & \\
\hline & 42_human_resour & 0.232 & & 0.086 & & 0.123 & প & 0.097 & & 0.101 & ষ & 0.095 & 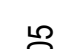 \\
\hline & 43_support_syst & 0.000 & & 0.036 & & 0.056 & 它 & 0.100 & 官 & 0.092 & م & 0.073 & 官 \\
\hline & 11_accur_info & 0.057 & & 0.218 & & 0.120 & & 0.149 & & 0.116 & & 0.141 & \\
\hline & 12_clear_info & 0.171 & & 0.104 & & 0.150 & & 0.123 & & 0.145 & & 0.137 & \\
\hline & 21_reliable_info & 0.032 & & 0.109 & & 0.088 & & 0.152 & & 0.123 & & 0.149 & \\
\hline & 22_timely_info & 0.194 & & 0.085 & & 0.068 & & 0.139 & & 0.134 & & 0.118 & \\
\hline & 23_language & 0.004 & & 0.052 & & 0.042 & & 0.012 & & 0.045 & & 0.023 & \\
\hline & 31_develop_time & 0.005 & & 0.054 & & 0.052 & & 0.024 & & 0.061 & & 0.037 & \\
\hline & 32_costs & 0.212 & & 0.049 & & 0.048 & & 0.065 & & 0.041 & & 0.043 & \\
\hline & 41_sust_financing & 0.000 & & 0.094 & & 0.147 & & 0.053 & & 0.042 & & 0.091 & \\
\hline 苑 & 42_human_resour & 0.227 & & 0.084 & & 0.120 & & 0.094 & $\check{x}$ & 0.099 & $\infty$ & 0.092 & 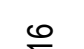 \\
\hline - & 43_support_syst & 0.000 & 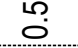 & 0.037 & مُ & 0.058 & 0 & 0.104 & $\ddot{0}$ & 0.095 & 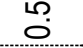 & 0.076 & $\mathscr{0}$ \\
\hline & 11_accur_info & 0.061 & & 0.236 & & 0.130 & & 0.161 & & 0.125 & & 0.152 & \\
\hline & 12_clear_info & 0.185 & & 0.113 & & 0.163 & & 0.133 & & 0.157 & & 0.149 & \\
\hline & 21_reliable_info & 0.031 & & 0.105 & & 0.085 & & 0.147 & & 0.119 & & 0.144 & \\
\hline & 22_timely_info & 0.190 & & 0.083 & & 0.067 & & 0.136 & & 0.131 & & 0.115 & \\
\hline & 23_language & 0.005 & & 0.070 & & 0.057 & & 0.017 & & 0.060 & & 0.032 & \\
\hline & 31_develop_time & 0.003 & & 0.032 & & 0.032 & & 0.015 & & 0.037 & & 0.022 & \\
\hline & 32_costs & 0.208 & & 0.049 & & 0.047 & & 0.064 & & 0.040 & & 0.042 & \\
\hline & 41_sust_financing & 0.000 & & 0.101 & & 0.157 & & 0.057 & & 0.045 & & 0.097 & \\
\hline ¿ & 42_human_resour & 0.222 & $\infty$ & 0.083 & $\bumpeq$ & 0.117 & న & 0.092 & 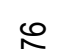 & 0.097 & 8 & 0.091 & ৫ \\
\hline $0_{1}^{\prime}$ & 43_support_syst & 0.000 & 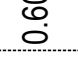 & 0.037 & $\stackrel{\circ}{\circ}$ & 0.058 & @ं & 0.104 & ש़ & 0.095 & Ð & 0.076 & : \\
\hline & 11_accur_info & 0.057 & & 0.222 & & 0.122 & & 0.151 & & 0.118 & & 0.143 & \\
\hline & 12_clear_info & 0.185 & & 0.113 & & 0.163 & & 0.133 & & 0.157 & & 0.149 & \\
\hline & 21_reliable_info & 0.031 & & 0.105 & & 0.085 & & 0.147 & & 0.119 & & 0.144 & \\
\hline & 22_timely_info & 0.189 & & 0.083 & & 0.067 & & 0.135 & & 0.131 & & 0.115 & \\
\hline & 23_language & 0.005 & & 0.071 & & 0.058 & & 0.017 & & 0.061 & & 0.032 & \\
\hline & 31_develop_time & 0.004 & & 0.045 & & 0.044 & & 0.020 & & 0.051 & & 0.031 & \\
\hline & 32_costs & 0.212 & & 0.049 & & 0.048 & & 0.065 & & 0.041 & & 0.043 & \\
\hline & 41_sust_financing & 0.000 & & 0.099 & & 0.154 & & 0.056 & & 0.044 & & 0.095 & \\
\hline 흥 & 42_human_resour & 0.239 & ৪ & 0.089 & $\bar{m}$ & 0.126 & $\mp$ & 0.100 & $œ$ & 0.104 & 용 & 0.098 & வ \\
\hline$\stackrel{q_{1}}{4}$ & 43_support_syst & 0.000 & 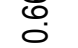 & 0.037 & @ִ & 0.058 & ڤே & 0.104 & $\stackrel{0}{0}$ & 0.095 & 0 & 0.076 & : \\
\hline
\end{tabular}




\begin{tabular}{|c|c|c|c|c|c|c|c|c|c|c|c|c|c|}
\hline \multirow[b]{2}{*}{ 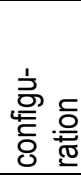 } & \multirow[b]{2}{*}{ objective } & \multicolumn{2}{|c|}{ G1A_EM_F } & \multicolumn{2}{|c|}{ G2A_HY_F } & \multicolumn{2}{|c|}{ G2B_HY_F } & \multicolumn{2}{|c|}{ G3A_HY_E } & \multicolumn{2}{|c|}{ G4A_EM_E } & \multicolumn{2}{|c|}{ G5A_AGRHYMET } \\
\hline & & 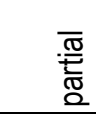 & 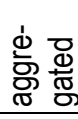 & 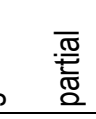 & 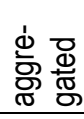 & 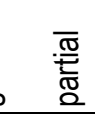 & 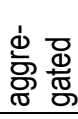 & 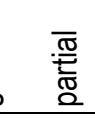 & 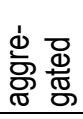 & 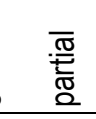 & 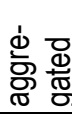 & 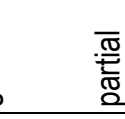 & 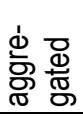 \\
\hline \multirow{10}{*}{ 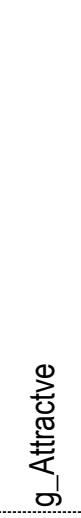 } & 11_accur_info & 0.061 & \multirow{10}{*}{$\begin{array}{l}8 \\
0 \\
0\end{array}$} & 0.236 & \multirow{10}{*}{$\begin{array}{l}\text { ণ্} \\
\text { ¿ }\end{array}$} & 0.130 & \multicolumn{2}{|r|}{0.161} & \multicolumn{2}{|r|}{0.125} & \multicolumn{2}{|r|}{0.152} & (ర) \\
\hline & 12_clear_info & 0.185 & & 0.113 & & 0.163 & \multicolumn{2}{|r|}{0.133} & \multicolumn{2}{|r|}{0.157} & \multicolumn{2}{|r|}{0.149} & \\
\hline & 21_reliable_info & 0.031 & & 0.105 & & 0.085 & \multicolumn{2}{|r|}{0.147} & \multicolumn{2}{|r|}{0.119} & & 0.144 & \\
\hline & 22_timely_info & 0.189 & & 0.082 & & 0.066 & & 0.135 & & 0.130 & & 0.114 & \\
\hline & 23_language & 0.005 & & 0.071 & & 0.058 & & 0.017 & & 0.061 & & 0.032 & \\
\hline & 31_develop_time & 0.003 & & 0.032 & & 0.032 & & 0.015 & & 0.037 & & 0.022 & \\
\hline & 32_costs & 0.208 & & 0.049 & & 0.047 & & 0.064 & & 0.040 & & 0.042 & \\
\hline & 41_sust_financing & 0.000 & & 0.102 & & 0.158 & & 0.058 & & 0.045 & & 0.098 & \\
\hline & 42_human_resour & 0.222 & & 0.083 & & 0.117 & ষ্ল & 0.092 & 浞 & 0.097 & & 0.091 & \\
\hline & 43_support_syst & 0.000 & & 0.037 & & 0.058 & بْ & 0.104 & 0 & 0.095 & 官 & 0.076 & ڤ્ \\
\hline & 11_accur_info & 0.061 & & 0.236 & & 0.130 & & 0.161 & & 0.125 & & 0.152 & \\
\hline & 12_clear_info & 0.185 & & 0.113 & & 0.163 & & 0.133 & & 0.157 & & 0.149 & \\
\hline & 21_reliable_info & 0.031 & & 0.105 & & 0.085 & & 0.147 & & 0.119 & & 0.144 & \\
\hline & 22_timely_info & 0.188 & & 0.082 & & 0.066 & & 0.134 & & 0.130 & & 0.114 & \\
\hline & 23_language & 0.005 & & 0.071 & & 0.058 & & 0.017 & & 0.061 & & 0.032 & \\
\hline & 31_develop_time & 0.003 & & 0.032 & & 0.032 & & 0.015 & & 0.037 & & 0.022 & \\
\hline & 32_costs & 0.208 & & 0.049 & & 0.047 & & 0.064 & & 0.040 & & 0.042 & \\
\hline & 41_sust_financing & 0.000 & & 0.098 & & 0.152 & & 0.055 & & 0.043 & & 0.094 & \\
\hline 言 & 42_human_resour & 0.224 & & 0.083 & & 0.118 & $\stackrel{2}{\longleftarrow}$ & 0.093 & $\hat{c}$ & 0.098 & & 0.092 & @ \\
\hline$c^{\prime}$ & 43_support_syst & 0.000 & ڤே. & 0.037 & @̈ & 0.058 & $\ddot{\circ}$ & 0.104 & ڤே. & 0.095 & ఝ. & 0.076 & 0 \\
\hline & 11_accur_info & 0.059 & & 0.229 & & 0.126 & & 0.156 & & 0.122 & & 0.148 & \\
\hline & 12_clear_info & 0.171 & & 0.104 & & 0.150 & & 0.123 & & 0.145 & & 0.137 & \\
\hline & 21_reliable_info & 0.031 & & 0.105 & & 0.085 & & 0.147 & & 0.119 & & 0.144 & \\
\hline & 22_timely_info & 0.190 & & 0.083 & & 0.067 & & 0.136 & & 0.131 & & 0.115 & \\
\hline & 23_language & 0.005 & & 0.068 & & 0.055 & & 0.016 & & 0.058 & & 0.031 & \\
\hline & 31_develop_time & 0.004 & & 0.049 & & 0.048 & & 0.022 & & 0.056 & & 0.034 & \\
\hline & 32_costs & 0.218 & & 0.051 & & 0.049 & & 0.067 & & 0.042 & & 0.044 & \\
\hline & 41_sust_financing & 0.000 & & 0.096 & & 0.149 & & 0.054 & & 0.043 & & 0.092 & \\
\hline 흠 & 42_human_resour & 0.244 & $\infty$ & 0.091 & m & 0.129 & 10 & 0.102 & $\approx$ & 0.106 & $\mathscr{O}$ & 0.100 & ষ্ণ \\
\hline$\underline{0}$ & 43_support_syst & 0.000 & ڤே & 0.037 & 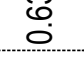 & 0.058 & 0 & 0.103 & 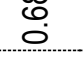 & 0.095 & 0 & 0.076 & 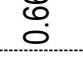 \\
\hline & 11_accur_info & 0.060 & & 0.232 & & 0.128 & & 0.158 & & 0.123 & & 0.150 & \\
\hline & 12_clear_info & 0.171 & & 0.104 & & 0.150 & & 0.123 & & 0.145 & & 0.137 & \\
\hline & 21_reliable_info & 0.031 & & 0.105 & & 0.085 & & 0.147 & & 0.119 & & 0.144 & \\
\hline & 22_timely_info & 0.192 & & 0.084 & & 0.068 & & 0.137 & & 0.133 & & 0.116 & \\
\hline & 23_language & 0.005 & & 0.068 & & 0.055 & & 0.016 & & 0.058 & & 0.031 & \\
\hline & 31_develop_time & 0.004 & & 0.049 & & 0.047 & & 0.022 & & 0.056 & & 0.034 & \\
\hline & 32_costs & 0.212 & & 0.049 & & 0.048 & & 0.065 & & 0.041 & & 0.043 & \\
\hline & 41_sust_financing & 0.000 & & 0.099 & & 0.154 & & 0.056 & & 0.044 & & 0.095 & \\
\hline 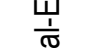 & 42_human_resour & 0.243 & $\infty$ & 0.090 & ₹ & 0.128 & $\infty$ & 0.101 & ৪ & 0.106 & 5 & 0.099 & $\mathscr{2}$ \\
\hline$u_{1}$ & 43_support_syst & 0.000 & @ே & 0.037 & @ே & 0.058 & @o & 0.103 & $\stackrel{0}{0}$ & 0.095 & @̋ & 0.076 & ڤ్ \\
\hline
\end{tabular}




\begin{tabular}{|c|c|c|c|c|c|c|c|c|c|c|c|c|c|}
\hline \multirow[b]{2}{*}{ 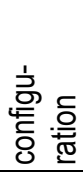 } & \multirow[b]{2}{*}{ objective } & \multicolumn{2}{|c|}{ G1A_EM_F } & \multicolumn{2}{|c|}{ G2A_HY_F } & \multicolumn{2}{|c|}{ G2B_HY_F } & \multicolumn{2}{|c|}{ G3A_HY_E } & \multicolumn{2}{|c|}{ G4A_EM_E } & \multicolumn{2}{|c|}{ G5A_AGRHYMET } \\
\hline & & 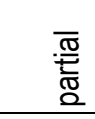 & 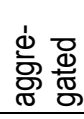 & 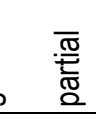 & 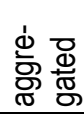 & 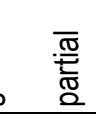 & 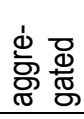 & 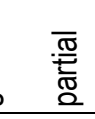 & $\begin{array}{l}d \\
\frac{d}{\sigma} \\
\frac{\Phi}{\pi} \\
\end{array}$ & 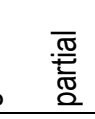 & 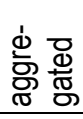 & 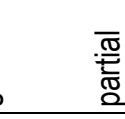 & 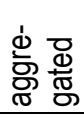 \\
\hline \multirow{10}{*}{ 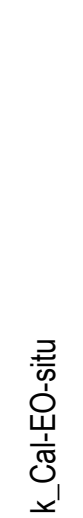 } & 11_accur_info & 0.063 & & 0.242 & & 0.133 & & 0.165 & & 0.128 & & 0.156 & \\
\hline & 12_clear_info & 0.171 & & 0.104 & & 0.150 & & 0.123 & & 0.145 & & 0.137 & \\
\hline & 21_reliable_info & 0.031 & & 0.105 & & 0.085 & & 0.147 & & 0.119 & & 0.144 & \\
\hline & 22_timely_info & 0.190 & & 0.083 & & 0.067 & & 0.136 & & 0.131 & & 0.115 & \\
\hline & 23_language & 0.005 & & 0.068 & & 0.055 & & 0.016 & & 0.058 & & 0.031 & \\
\hline & 31_develop_time & 0.004 & & 0.047 & & 0.046 & & 0.021 & & 0.054 & & 0.033 & \\
\hline & 32_costs & 0.208 & & 0.049 & & 0.047 & & 0.064 & & 0.040 & & 0.042 & \\
\hline & 41_sust_financing & 0.000 & & 0.098 & & 0.152 & & 0.055 & & 0.043 & & 0.094 & \\
\hline & 42_human_resour & 0.241 & \multirow{2}{*}{$\begin{array}{l}\widetilde{\widetilde{C}} \\
\stackrel{0}{0}\end{array}$} & 0.090 & هి & 0.127 & \multirow{2}{*}{$\begin{array}{l}8 \\
\bigotimes \\
0\end{array}$} & 0.100 & $\infty$ & 0.105 & \multirow{2}{*}{$\begin{array}{l}\text { U⿺ } \\
00 \\
0\end{array}$} & 0.098 & 寸 \\
\hline & 43_support_syst & 0.000 & & 0.037 & @્ & 0.058 & & 0.103 & @ & 0.095 & & 0.076 & @ \\
\hline
\end{tabular}

Table SI-34. Results of the MCDA with uncertainty. MCDA values resulting from Monte Carlo simulation of uncertainty on predictions with 1'000 runs. For each stakeholder (e.g., G1A_EM_F, etc.) and each configuration (a_Fast-dev, etc.), the calculated mean and median value are presented with parameters of the according uncertainty distribution.

\begin{tabular}{|c|c|c|c|c|c|c|c|c|c|c|c|c|}
\hline & & $\begin{array}{l}\frac{1}{\omega} \\
\stackrel{w}{\omega} \\
\stackrel{\omega}{\prime}^{\prime}\end{array}$ & 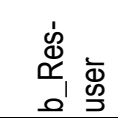 & 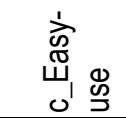 & 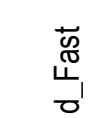 & 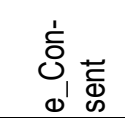 & $\begin{array}{l}\dot{2} \\
\frac{0}{4} \\
\end{array}$ & 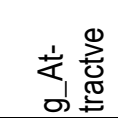 & $\begin{array}{l}\stackrel{0}{\bar{z}} \\
\stackrel{y^{\prime}}{c^{\prime}}\end{array}$ & 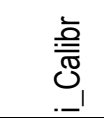 & 悥 & 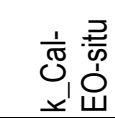 \\
\hline \multirow{6}{*}{ 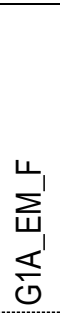 } & mean value & 0.652 & 0.651 & 0.633 & 0.584 & 0.604 & 0.666 & 0.600 & 0.599 & 0.652 & 0.638 & 0.615 \\
\hline & 0.05 quantile & 0.639 & 0.635 & 0.613 & 0.566 & 0.588 & 0.644 & 0.585 & 0.584 & 0.632 & 0.620 & 0.596 \\
\hline & 0.95 quantile & 0.666 & 0.666 & 0.653 & 0.606 & 0.619 & 0.688 & 0.615 & 0.615 & 0.674 & 0.658 & 0.632 \\
\hline & median value & 0.651 & 0.652 & 0.633 & 0.583 & 0.604 & 0.666 & 0.600 & 0.598 & 0.652 & 0.638 & 0.615 \\
\hline & 0.25 quartile & 0.646 & 0.645 & 0.624 & 0.576 & 0.597 & 0.657 & 0.594 & 0.593 & 0.644 & 0.630 & 0.607 \\
\hline & 0.75 quartile & 0.657 & 0.659 & 0.642 & 0.592 & 0.610 & 0.675 & 0.606 & 0.605 & 0.661 & 0.647 & 0.621 \\
\hline \multirow{6}{*}{ 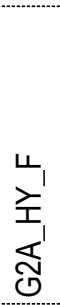 } & mean value & 0.408 & 0.616 & 0.566 & 0.525 & 0.581 & 0.609 & 0.584 & 0.574 & 0.596 & 0.606 & 0.608 \\
\hline & 0.05 quantile & 0.402 & 0.586 & 0.545 & 0.505 & 0.544 & 0.585 & 0.550 & 0.540 & 0.568 & 0.578 & 0.573 \\
\hline & 0.95 quantile & 0.415 & 0.646 & 0.588 & 0.545 & 0.617 & 0.633 & 0.618 & 0.609 & 0.622 & 0.634 & 0.642 \\
\hline & median value & 0.408 & 0.616 & 0.566 & 0.525 & 0.582 & 0.609 & 0.585 & 0.574 & 0.597 & 0.606 & 0.609 \\
\hline & 0.25 quartile & 0.405 & 0.604 & 0.557 & 0.516 & 0.567 & 0.599 & 0.570 & 0.559 & 0.585 & 0.592 & 0.594 \\
\hline & 0.75 quartile & 0.411 & 0.628 & 0.574 & 0.534 & 0.596 & 0.620 & 0.599 & 0.590 & 0.608 & 0.617 & 0.622 \\
\hline \multirow{6}{*}{ 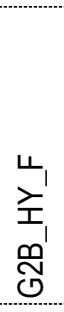 } & mean value & 0.408 & 0.645 & 0.604 & 0.547 & 0.607 & 0.652 & 0.612 & 0.597 & 0.616 & 0.627 & 0.622 \\
\hline & 0.05 quantile & 0.402 & 0.621 & 0.583 & 0.528 & 0.575 & 0.629 & 0.582 & 0.568 & 0.593 & 0.605 & 0.595 \\
\hline & 0.95 quantile & 0.414 & 0.667 & 0.625 & 0.566 & 0.636 & 0.673 & 0.639 & 0.625 & 0.636 & 0.649 & 0.646 \\
\hline & median value & 0.407 & 0.645 & 0.604 & 0.547 & 0.608 & 0.652 & 0.612 & 0.598 & 0.617 & 0.627 & 0.622 \\
\hline & 0.25 quartile & 0.405 & 0.636 & 0.596 & 0.539 & 0.596 & 0.643 & 0.600 & 0.584 & 0.607 & 0.618 & 0.612 \\
\hline & 0.75 quartile & 0.410 & 0.655 & 0.612 & 0.555 & 0.620 & 0.661 & 0.624 & 0.609 & 0.626 & 0.636 & 0.632 \\
\hline \multirow{6}{*}{ 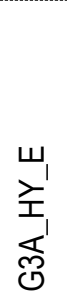 } & mean value & 0.444 & 0.670 & 0.609 & 0.612 & 0.646 & 0.666 & 0.645 & 0.638 & 0.650 & 0.654 & 0.653 \\
\hline & 0.05 quantile & 0.437 & 0.637 & 0.583 & 0.586 & 0.611 & 0.635 & 0.612 & 0.605 & 0.616 & 0.622 & 0.618 \\
\hline & 0.95 quantile & 0.453 & 0.701 & 0.637 & 0.636 & 0.680 & 0.695 & 0.677 & 0.670 & 0.678 & 0.686 & 0.686 \\
\hline & median value & 0.444 & 0.671 & 0.609 & 0.613 & 0.646 & 0.667 & 0.646 & 0.638 & 0.651 & 0.655 & 0.653 \\
\hline & 0.25 quartile & 0.441 & 0.657 & 0.597 & 0.602 & 0.633 & 0.653 & 0.631 & 0.623 & 0.637 & 0.640 & 0.638 \\
\hline & 0.75 quartile & 0.448 & 0.685 & 0.620 & 0.624 & 0.661 & 0.680 & 0.661 & 0.652 & 0.663 & 0.669 & 0.668 \\
\hline
\end{tabular}




\begin{tabular}{|c|c|c|c|c|c|c|c|c|c|c|c|c|}
\hline & & $\begin{array}{l}\frac{1}{\omega} \\
\underset{\sigma}{\sigma} \\
\leftarrow^{\prime} \\
\sigma\end{array}$ & 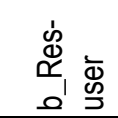 & 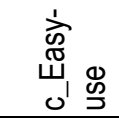 & 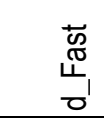 & 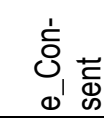 & 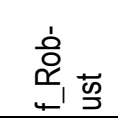 & 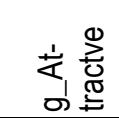 & $\begin{array}{l}\text { 음 } \\
\stackrel{\tilde{w}^{\prime}}{c^{\prime}}\end{array}$ & 产 & 递 & 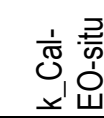 \\
\hline \multirow{6}{*}{$\begin{array}{l}\sum^{\prime} \\
\text { W' } \\
\text { 志 }\end{array}$} & mean value & 0.467 & 0.644 & 0.594 & 0.565 & 0.593 & 0.642 & 0.593 & 0.588 & 0.621 & 0.624 & 0.619 \\
\hline & 0.05 quantile & 0.460 & 0.616 & 0.573 & 0.544 & 0.556 & 0.617 & 0.559 & 0.555 & 0.594 & 0.599 & 0.591 \\
\hline & 0.95 quantile & 0.475 & 0.669 & 0.617 & 0.585 & 0.625 & 0.666 & 0.625 & 0.620 & 0.644 & 0.649 & 0.646 \\
\hline & median value & 0.466 & 0.645 & 0.594 & 0.565 & 0.594 & 0.643 & 0.594 & 0.588 & 0.622 & 0.624 & 0.619 \\
\hline & 0.25 quartile & 0.464 & 0.633 & 0.585 & 0.556 & 0.580 & 0.632 & 0.579 & 0.575 & 0.611 & 0.613 & 0.607 \\
\hline & 0.75 quartile & 0.470 & 0.656 & 0.603 & 0.575 & 0.607 & 0.653 & 0.608 & 0.602 & 0.632 & 0.636 & 0.631 \\
\hline \multirow{6}{*}{ 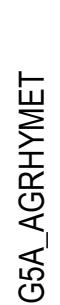 } & mean value & 0.407 & 0.661 & 0.603 & 0.588 & 0.632 & 0.658 & 0.633 & 0.622 & 0.632 & 0.641 & 0.639 \\
\hline & 0.05 quantile & 0.401 & 0.628 & 0.576 & 0.562 & 0.596 & 0.628 & 0.599 & 0.587 & 0.600 & 0.610 & 0.605 \\
\hline & 0.95 quantile & 0.415 & 0.690 & 0.631 & 0.611 & 0.666 & 0.686 & 0.666 & 0.655 & 0.659 & 0.671 & 0.672 \\
\hline & median value & 0.407 & 0.662 & 0.603 & 0.588 & 0.633 & 0.660 & 0.633 & 0.622 & 0.634 & 0.641 & 0.639 \\
\hline & 0.25 quartile & 0.404 & 0.648 & 0.592 & 0.577 & 0.619 & 0.646 & 0.619 & 0.608 & 0.620 & 0.627 & 0.625 \\
\hline & 0.75 quartile & 0.410 & 0.675 & 0.614 & 0.600 & 0.647 & 0.672 & 0.648 & 0.637 & 0.646 & 0.655 & 0.654 \\
\hline
\end{tabular}
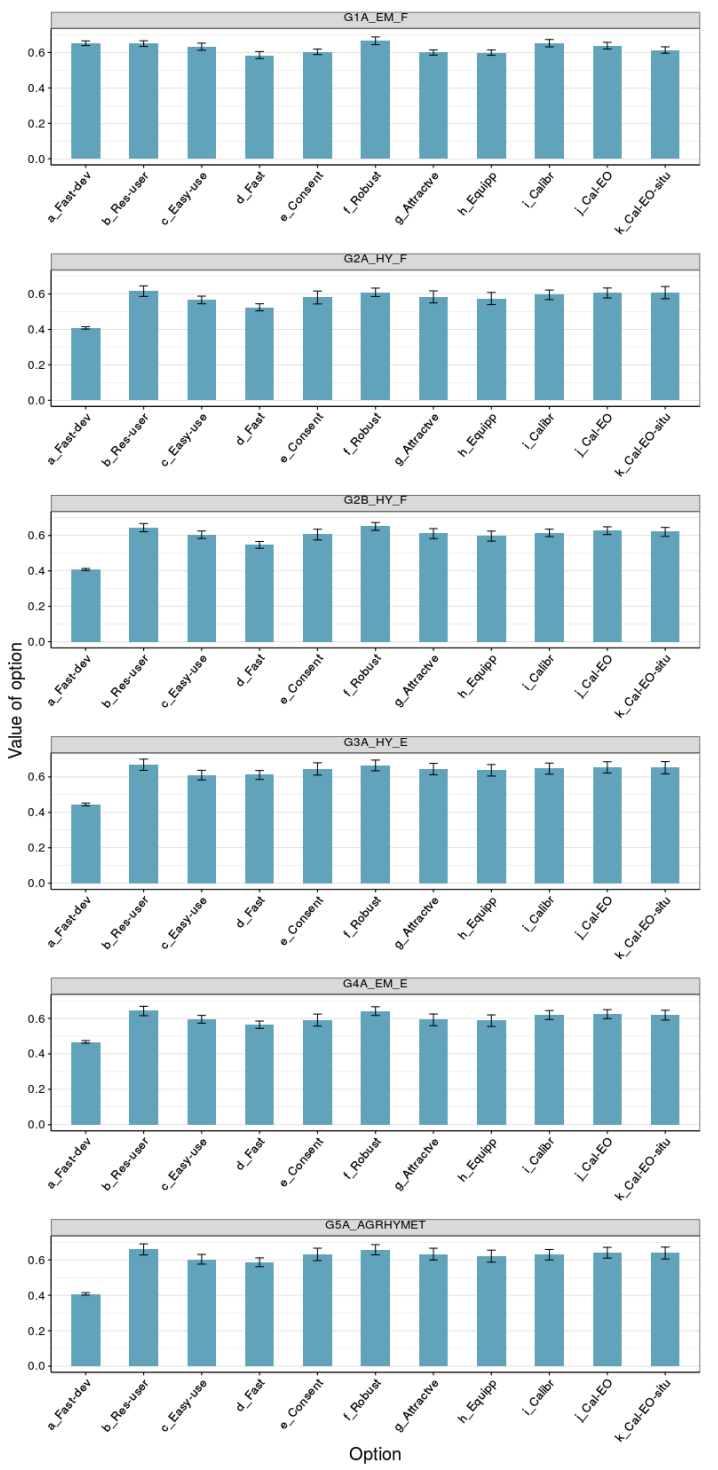

Figure SI-34. MCDA results with uncertainty. Mean (average) total value ( $y$-axis) calculated by the MCDA for each configuration ( $x$ axis) and each stakeholder (boxes). Uncertainty of the predictions is included with 1'000 Monte Carlo simulation runs of the MCDA for each stakeholder and each configuration. The mean overall value ( $y$-axis) can be anywhere between 0 (none of the objectives are achieved at all) and 1 (all objectives are fully achieved). Error bars show the 0.05 and 0.95 quantiles of the overall value. 

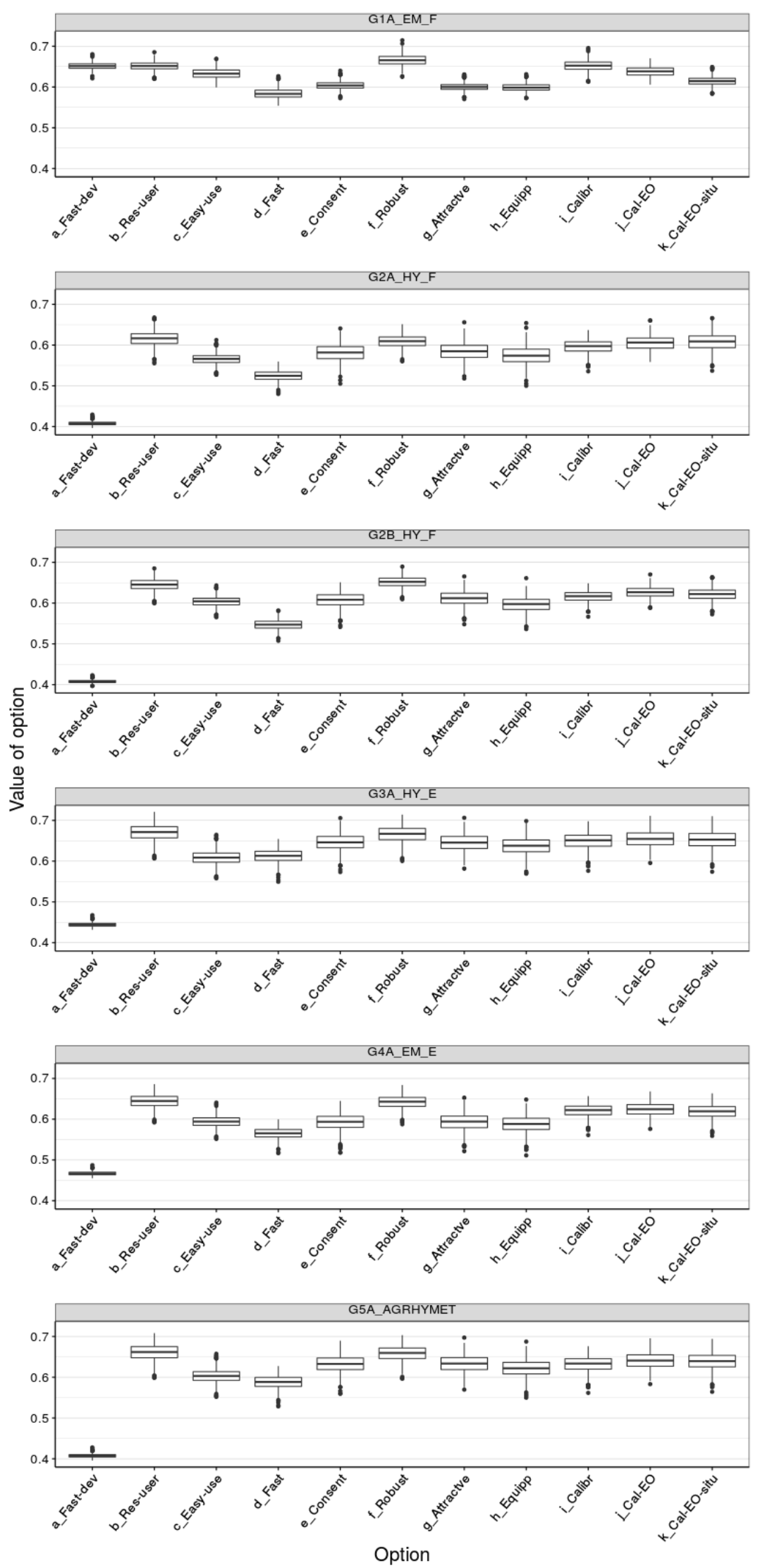

Figure SI-35. MCDA results with uncertainty: Median overall (total) value (y-axis) calculated by the MCDA for each configuration (xaxis) and each stakeholder (boxes). Uncertainty of the predictions is included with 1'000 Monte Carlo simulation runs of the MCDA calculation for each stakeholder and each configuration. The values (from 0 to 1 ) are displayed on the $y$-axis, where 0 means that none of the objectives are achieved and 1 that all objectives are fully achieved. The boxplots show the 0.25 (lower), 0.5 (median), and 0.75 (upper) quartiles of the values as result of the Monte Carlo simulation. 


\subsection{MCDA results: sensitivity analyses}

Table SI-35: Results of the sensitivity analyses. For each setting (column S0-S31), the mean total value and mean rank resulting from the 1'000 Monte Carlo simulation runs is provided for each stakeholder group (G1A_EM_F, etc.) and configuration (a_Fast-dev, etc.). Yellow highlight: ranking of our standard analysis (S0) compared with additive aggregation model (S11) for best-performing configurations across stakeholder groups $b_{-}$Res-user and $f$ _Robust; green highlight: rankings for the also well-performing configuration i_Calibr.

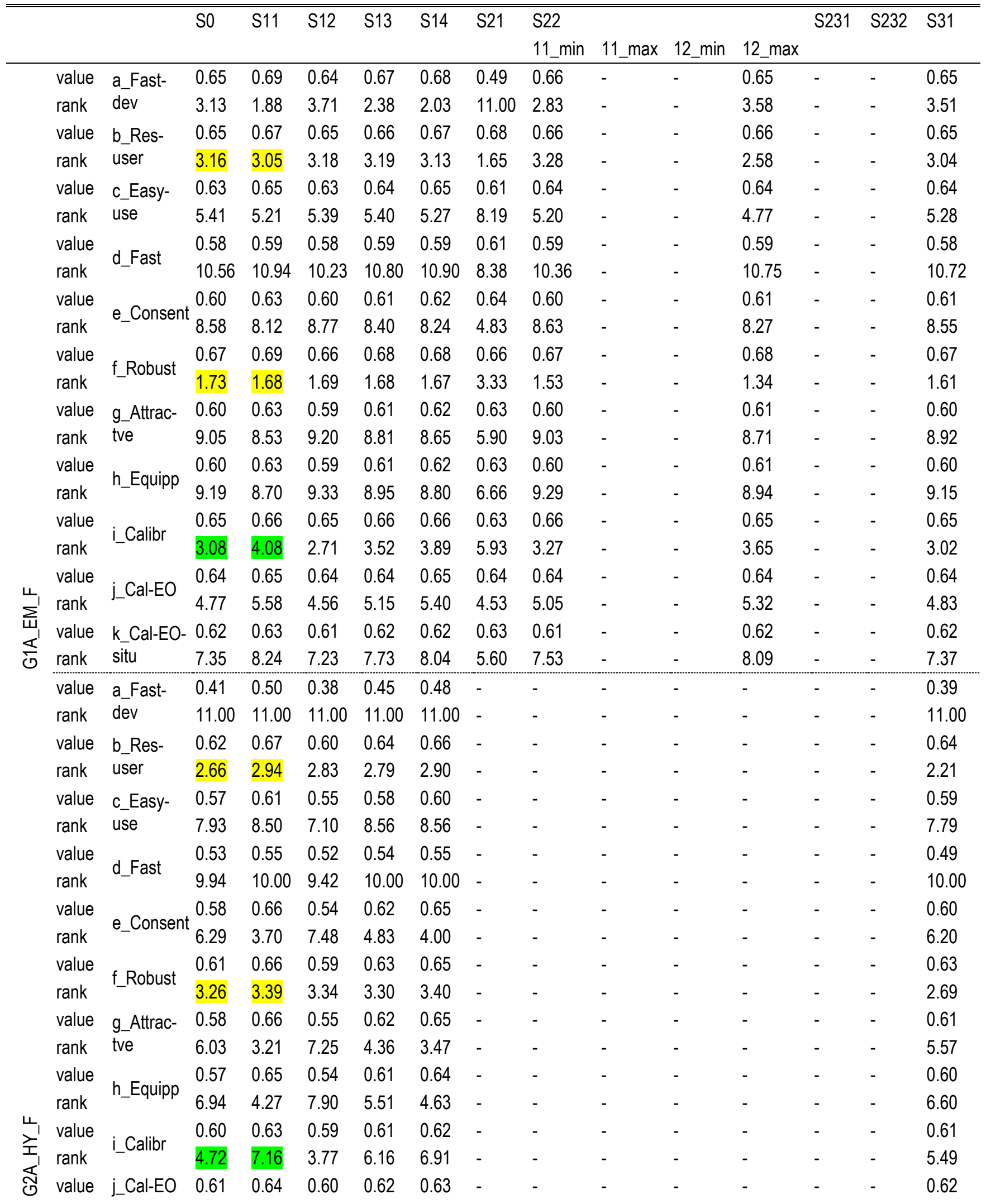




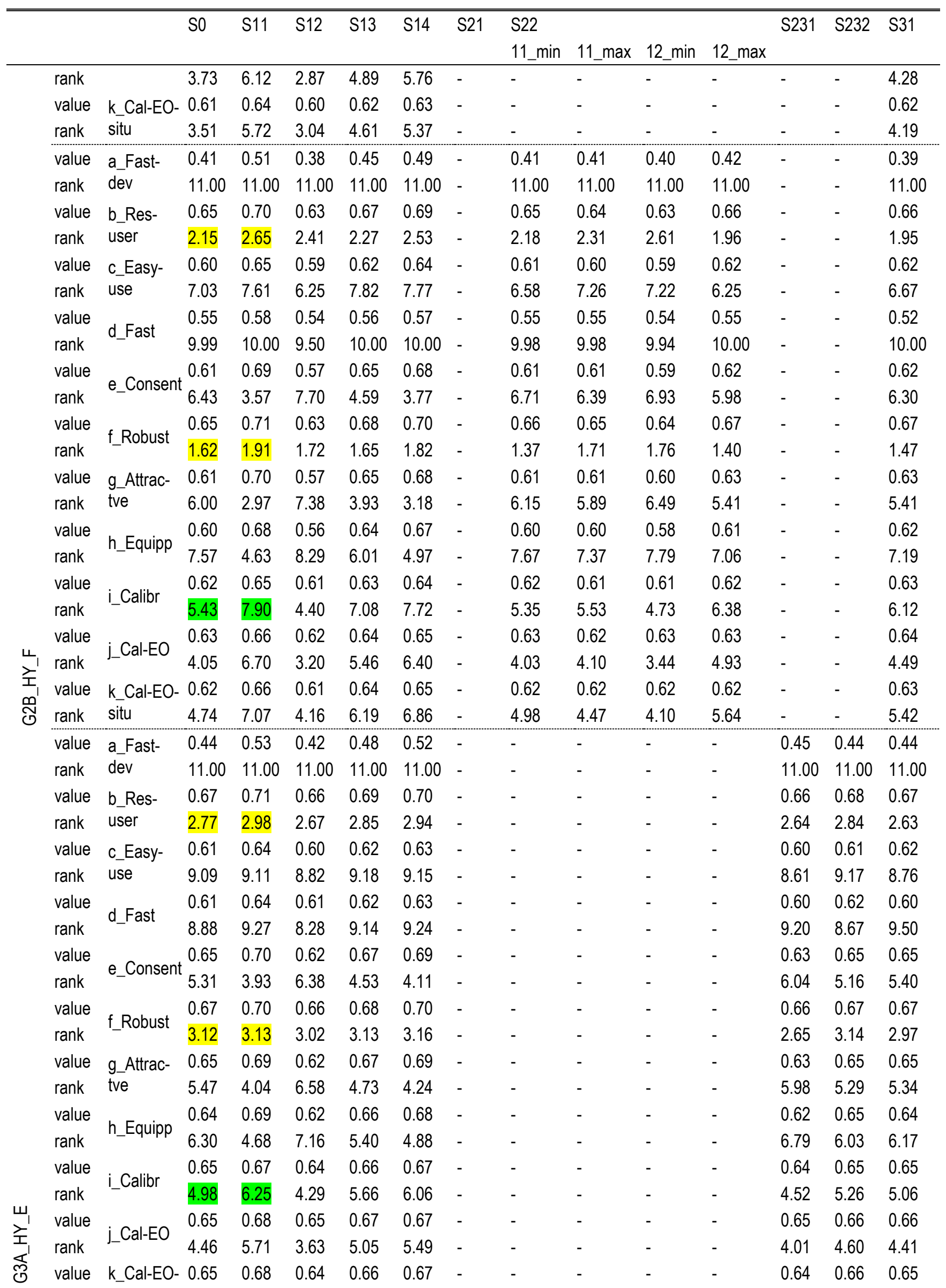




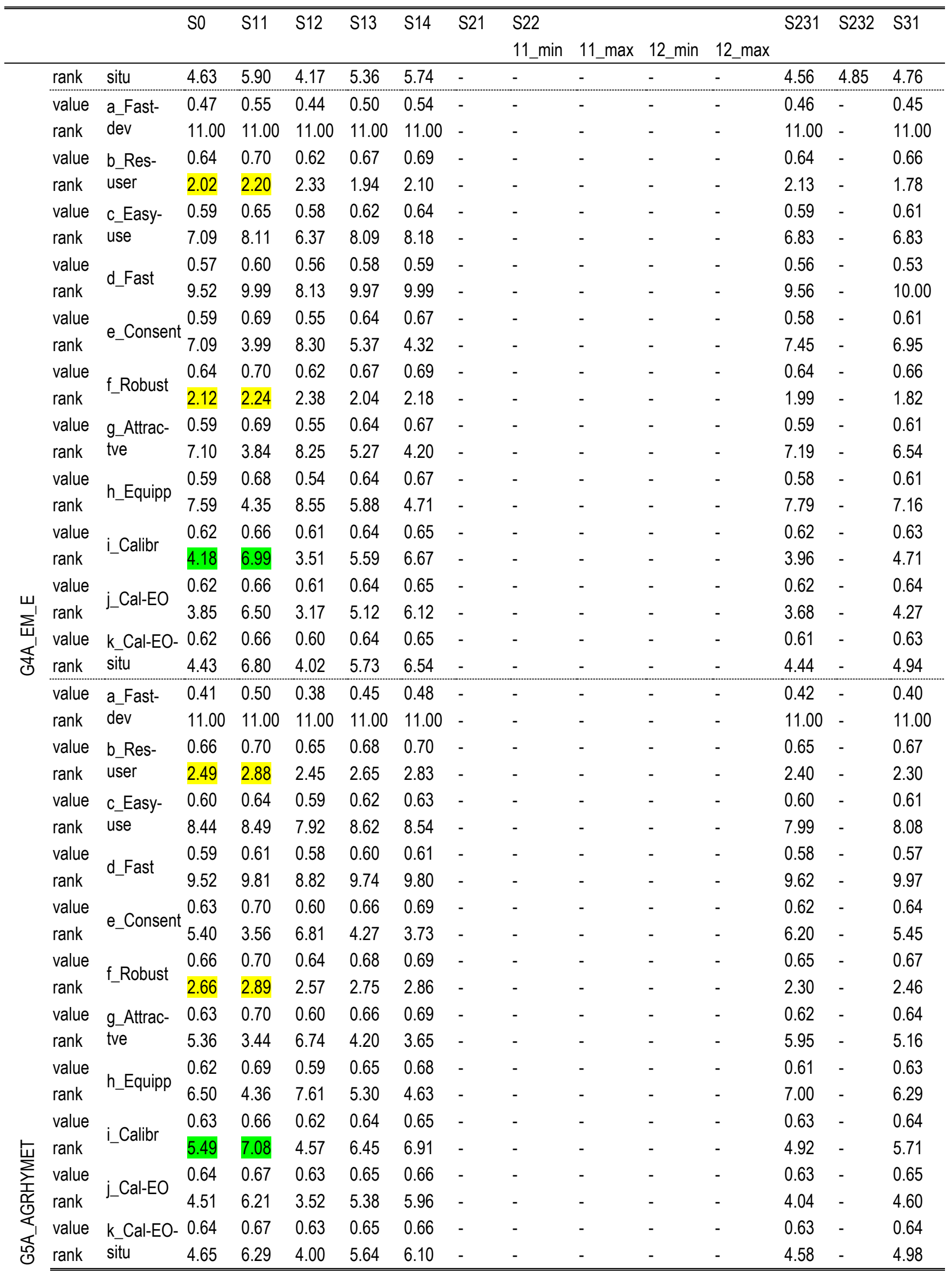


Table SI-36: Overview of Kendall's T rank correlation coefficient between ranks of configurations in the main MCDA (setting S0) and different settings for sensitivity analyses (S11 to S31), averaged (Aver) over all involved stakeholder groups (SH; e.g., group G1A).

\begin{tabular}{|c|c|c|c|c|}
\hline & & S11 (all SH) & S12 (all SH) & S13 (all SH) \\
\hline \multirow[t]{2}{*}{ Kendall's T } & Aver & 0.667 & 0.903 & 0.770 \\
\hline & & S14 (all SH) & $\mathrm{S} 21(\mathrm{G} 1 \mathrm{~A})$ & $S 22(G 1 A, G 2 A, G 2 B)$ \\
\hline \multirow[t]{2}{*}{ Kendall's T } & Aver & $\overline{0.685}$ & 0.309 & 0.955 \\
\hline & & S231 (G3A, G4A, G5A) & S232 (G3A, G4A, G5A) & S31 \\
\hline Kendall's T & Aver & 0.891 & 0.964 & 0.952 \\
\hline
\end{tabular}
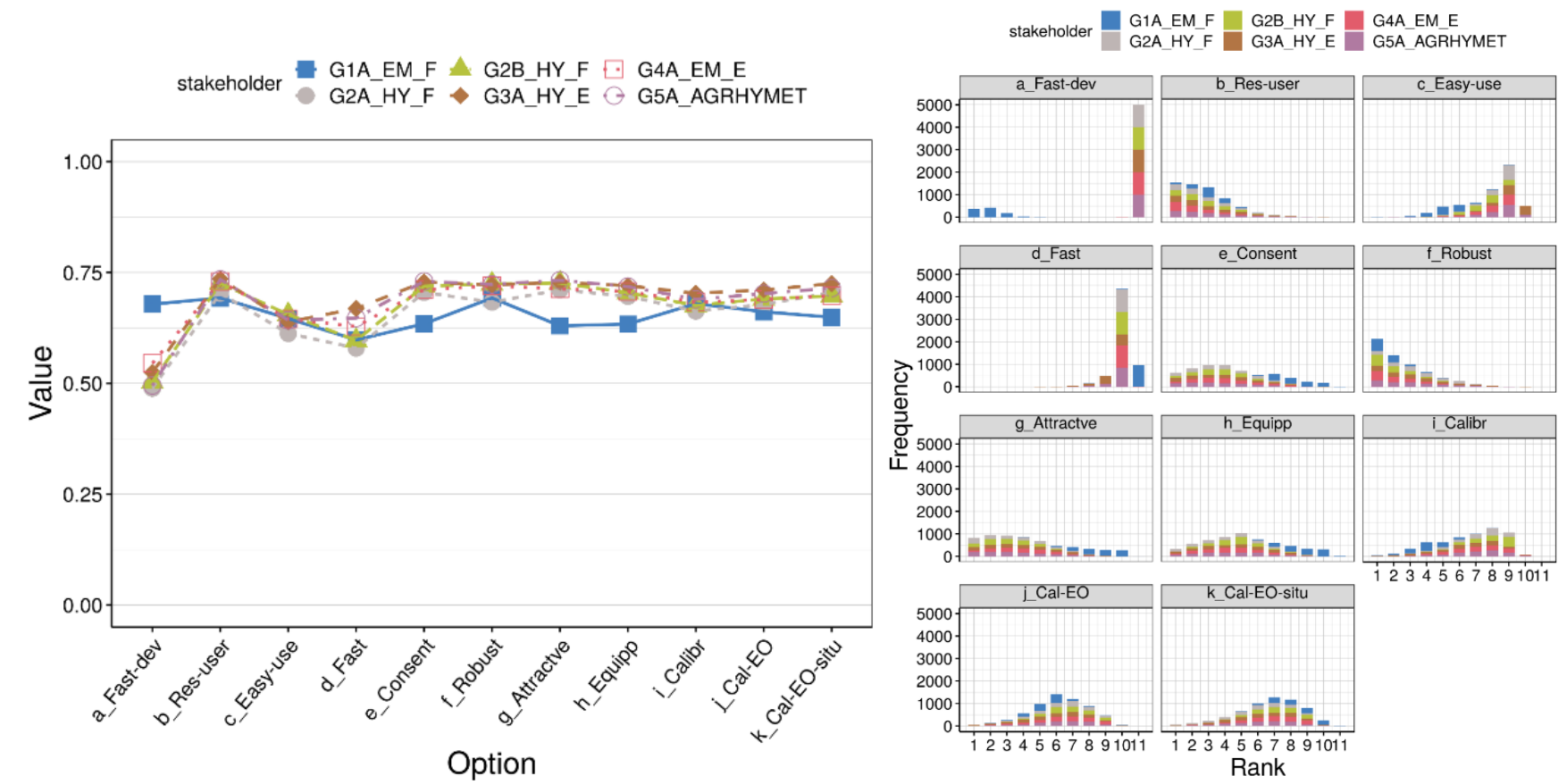

Figure SI-36: Results of the sensitivity analysis setting S11, additive model for all six stakeholder groups, $y=1$. 

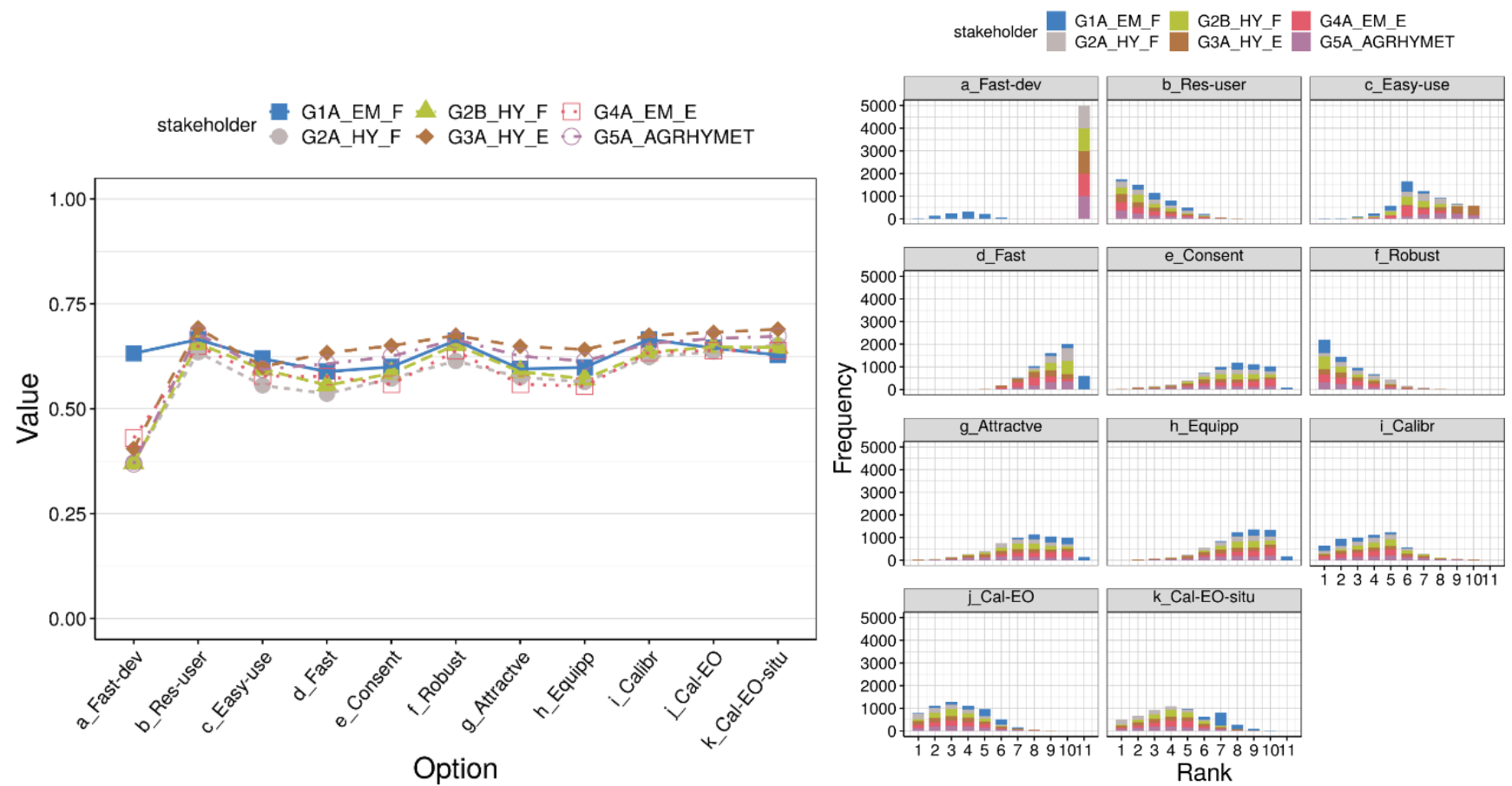

Figure SI-37: Results of the sensitivity analysis setting S12, weighted geometric mean for all six stakeholder groups, $y \rightarrow 0$.
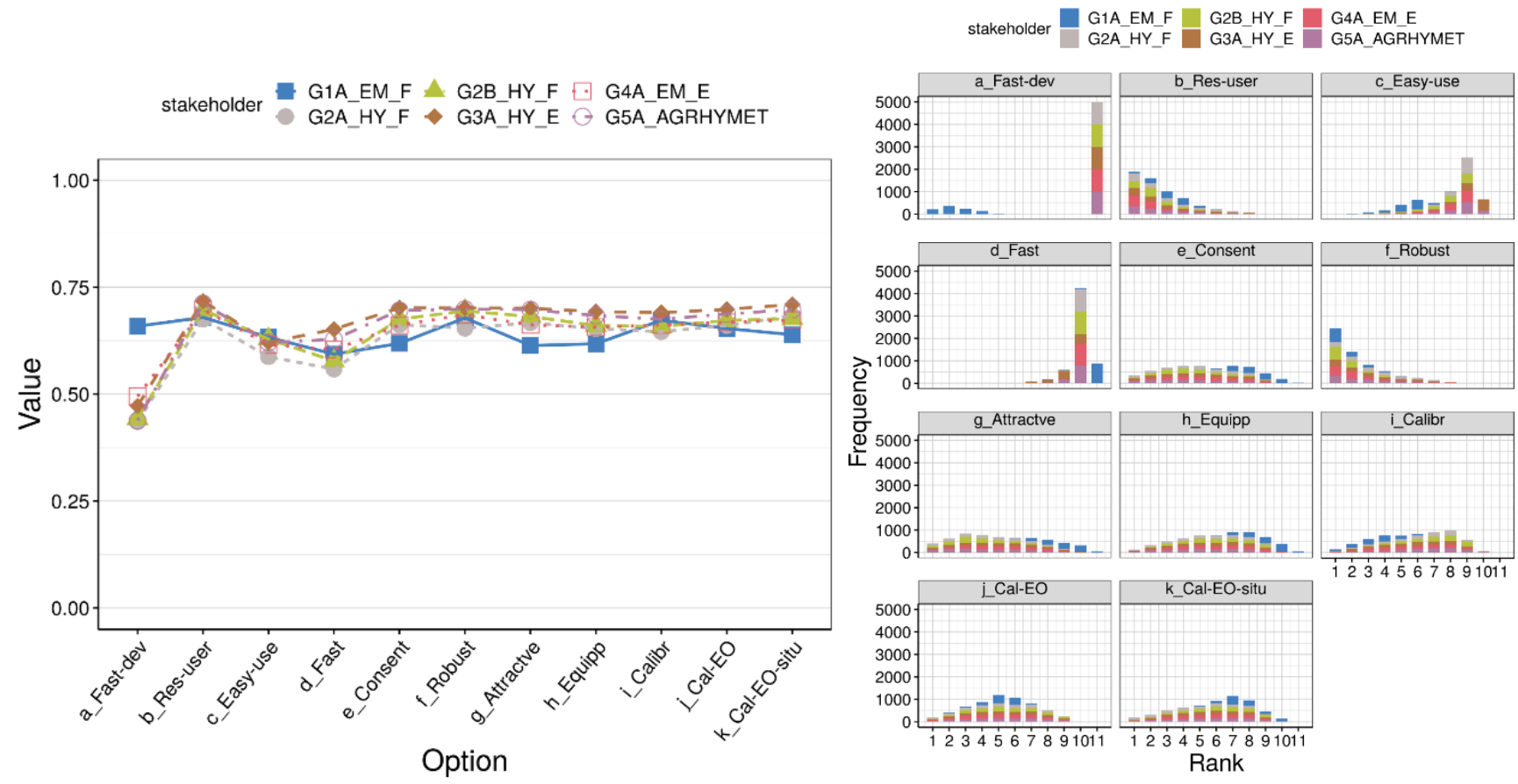

Figure SI-38: Results of the sensitivity analysis setting S13, mixture model for all six stakeholder groups, $y=0.5$ 

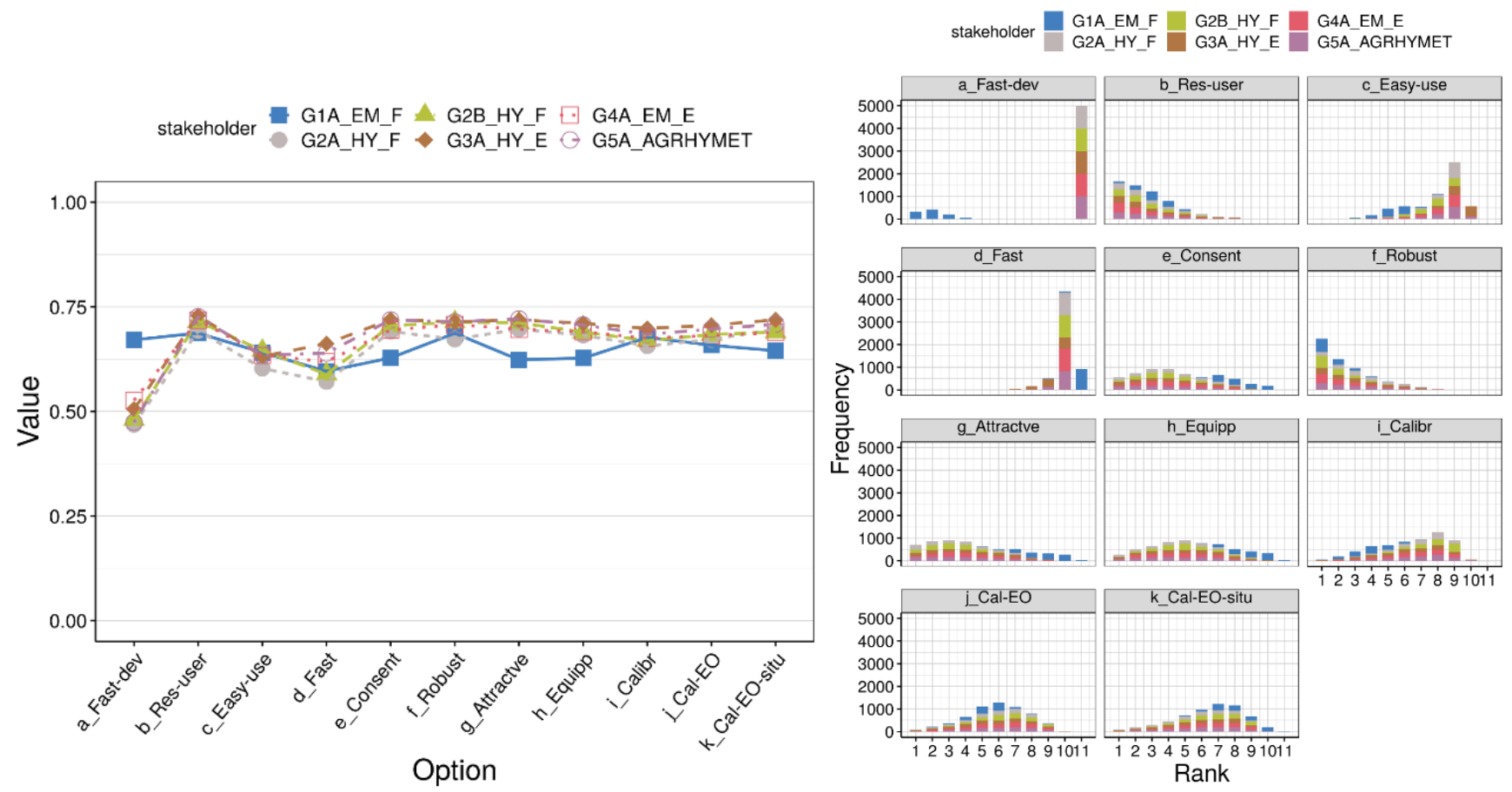

Figure SI-39: Results of sensitivity analysis setting S14, weighted power mean close to additive for all six stakeholder groups, $\mathrm{Y}=$ 0.8 .
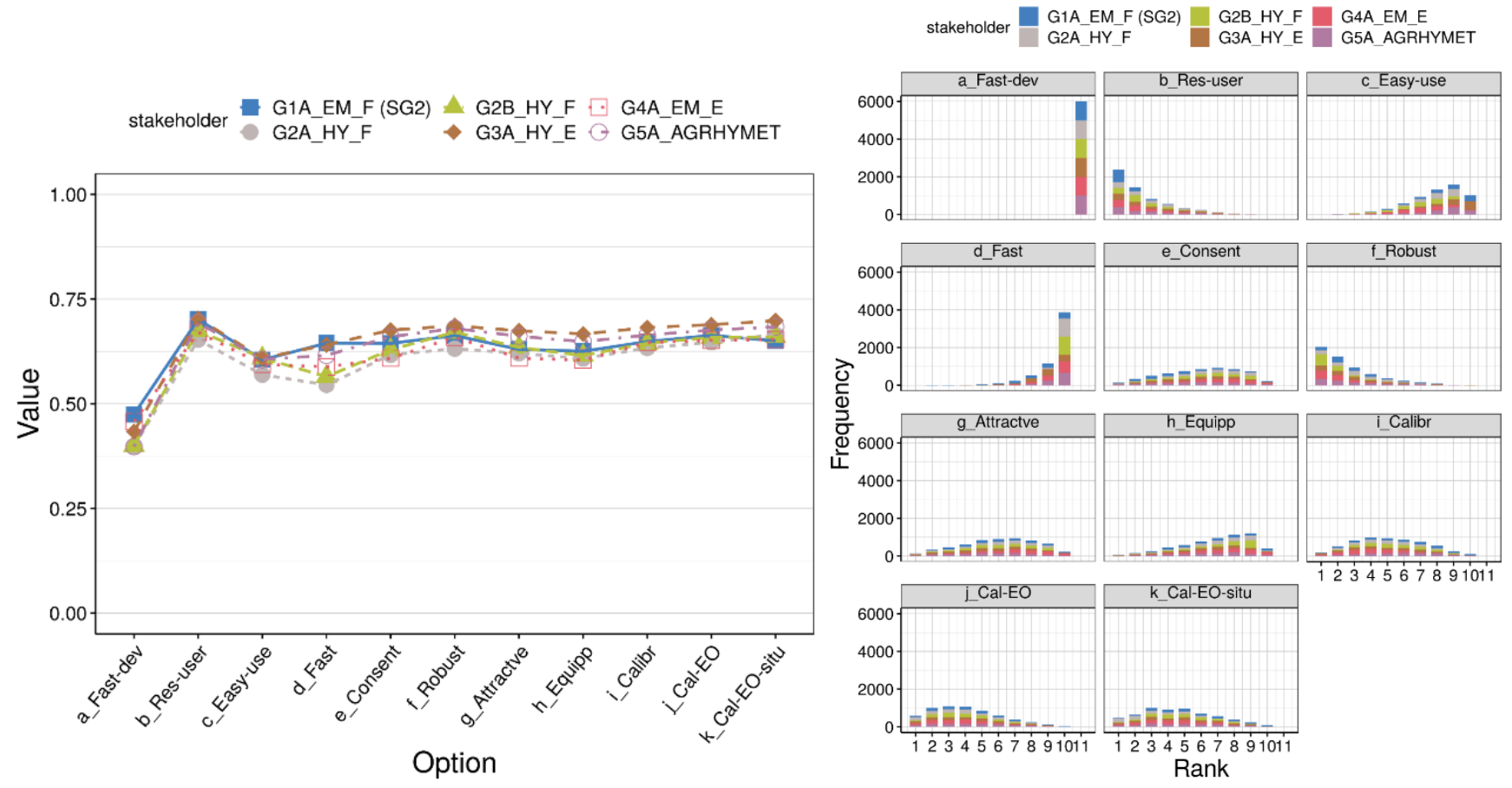

Figure SI-40: Results of the sensitivity analysis setting S21, alternative weight set for group G1A, resulting from consistency check. 

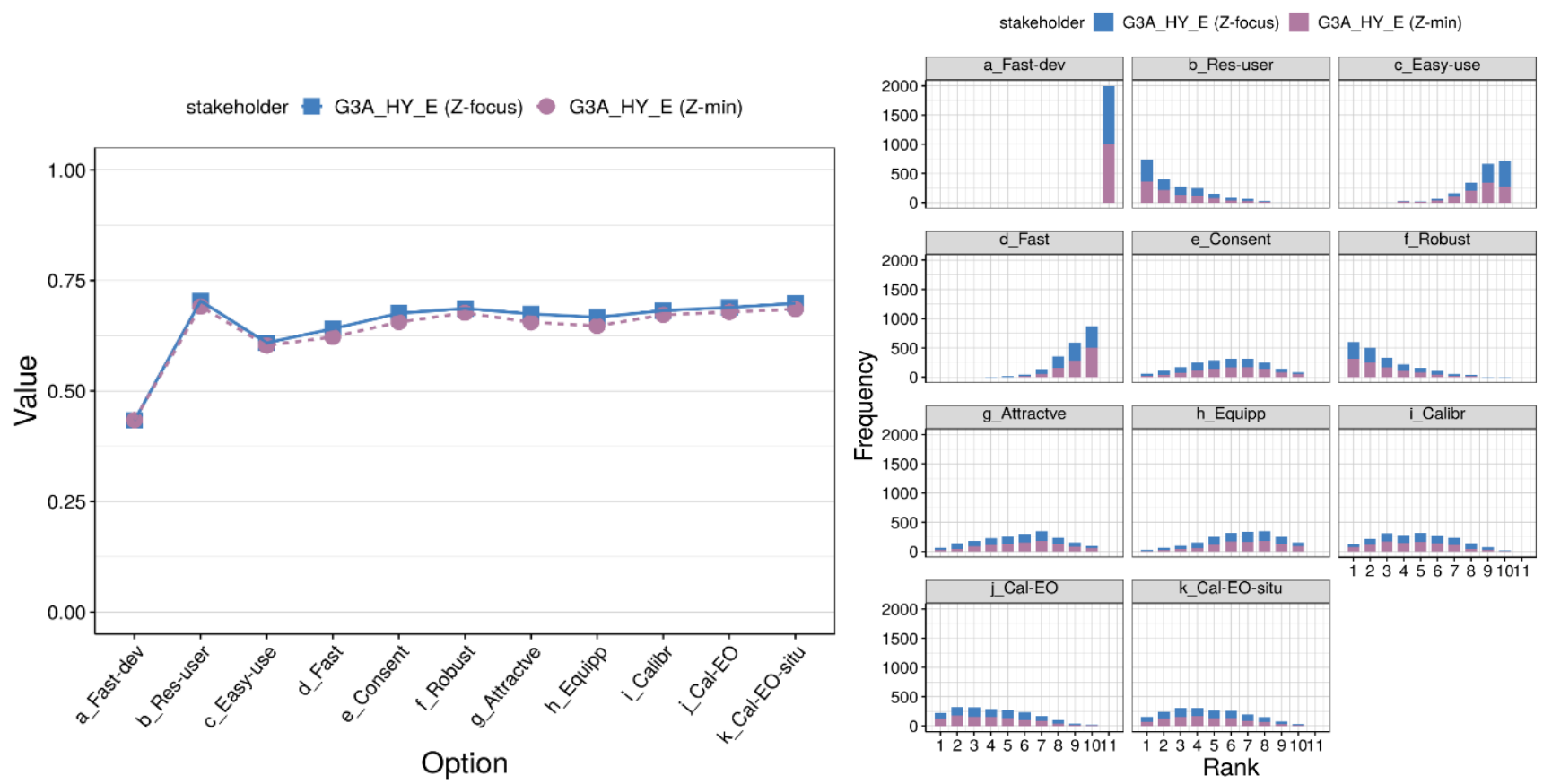

Figure SI-41: Results of the sensitivity analysis setting S231 for group G3A_HY_E, resulting from ranges assigned to Z min.
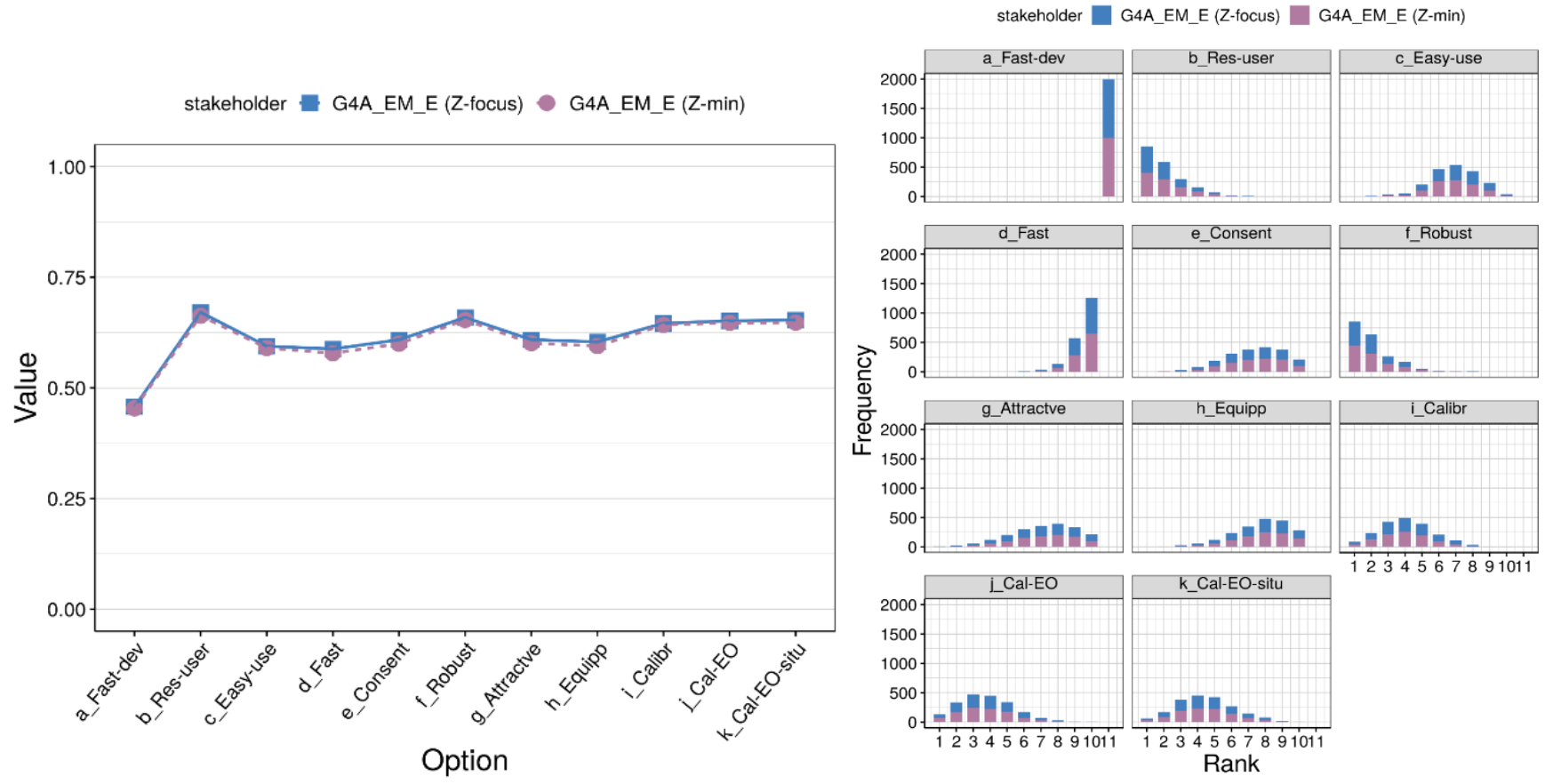

Figure SI-42: Results of the sensitivity analysis setting S231 for group G4A_EM_E, resulting from ranges assigned to Z min. 
stakeholder G5A AGRHYMET (Z-foucs) G5A AGRHYMET (Z-min)

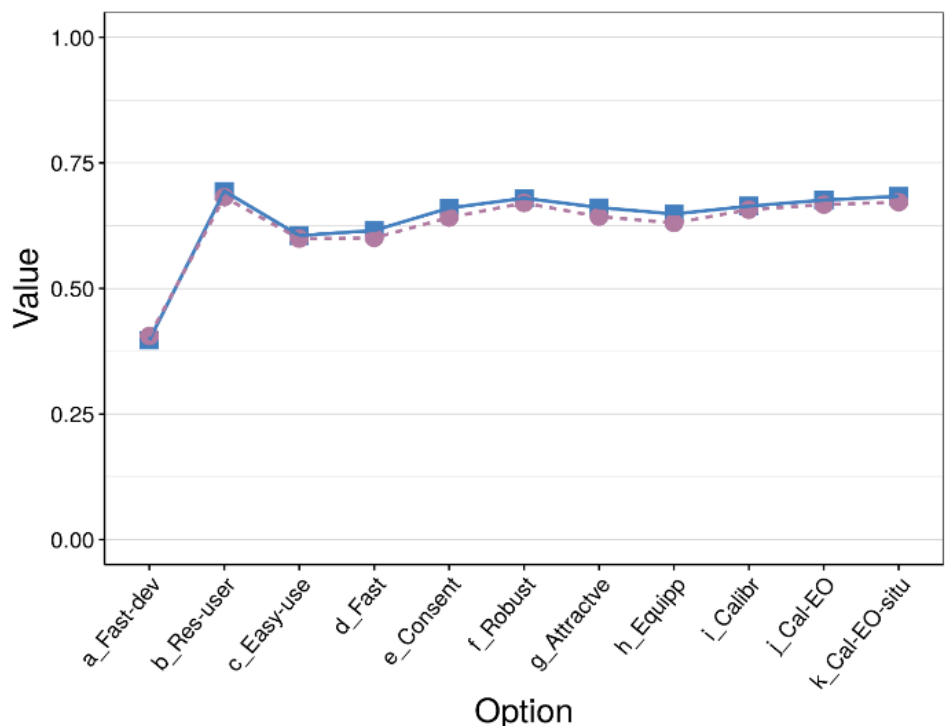

stakeholder G5A_AGRHYMET (Z-foucs) — G5A_AGRHYMET (Z-min)
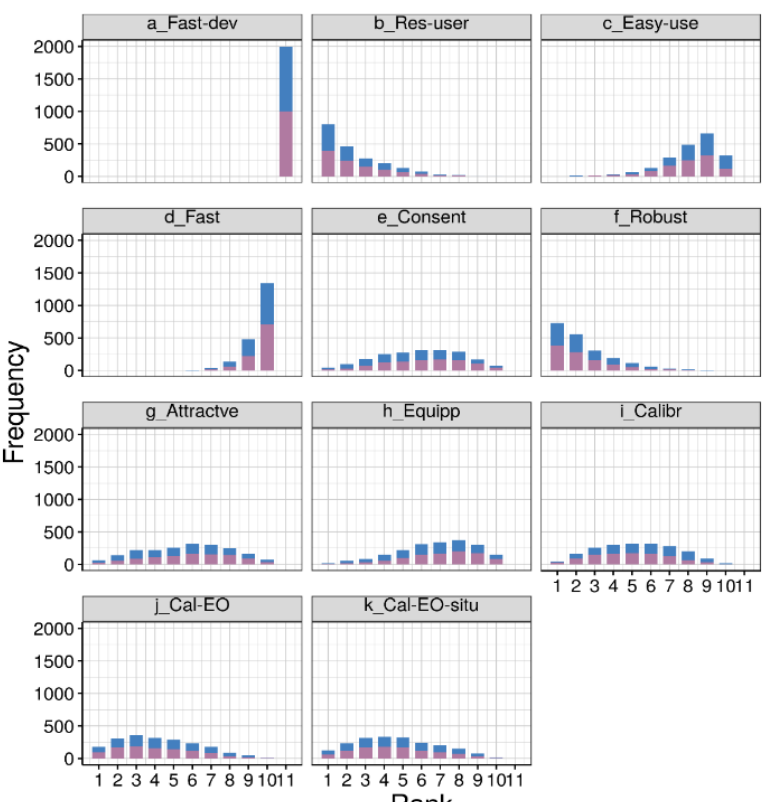

Rank

Figure SI-43: Results of the sensitivity analysis setting S231 for group G5A_AGRHYMET, resulting from ranges assigned to Z min.
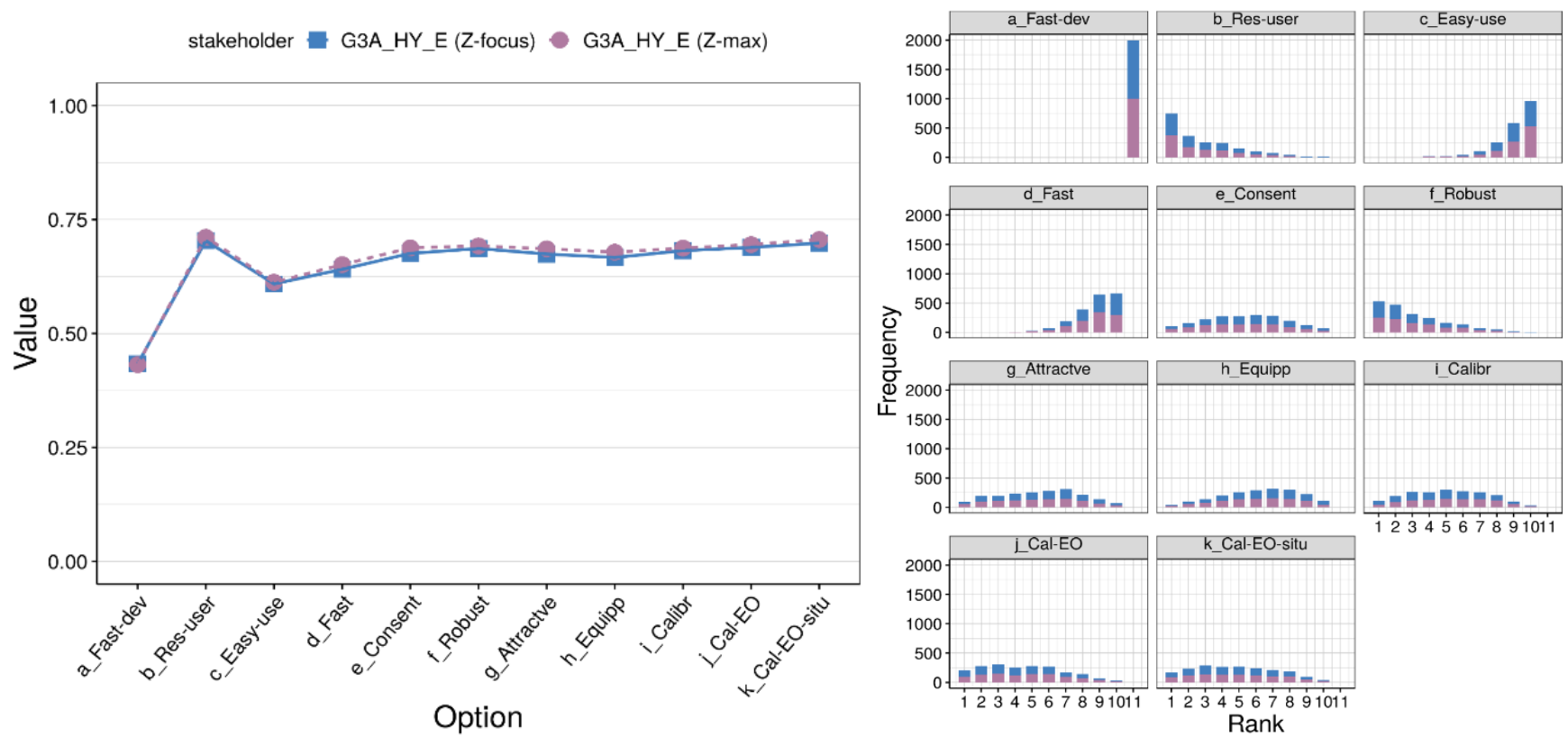

Rank

Figure SI-44: Results of the sensitivity analysis setting S232 for group G3A_HY_E, resulting from ranges assigned to Z max. 

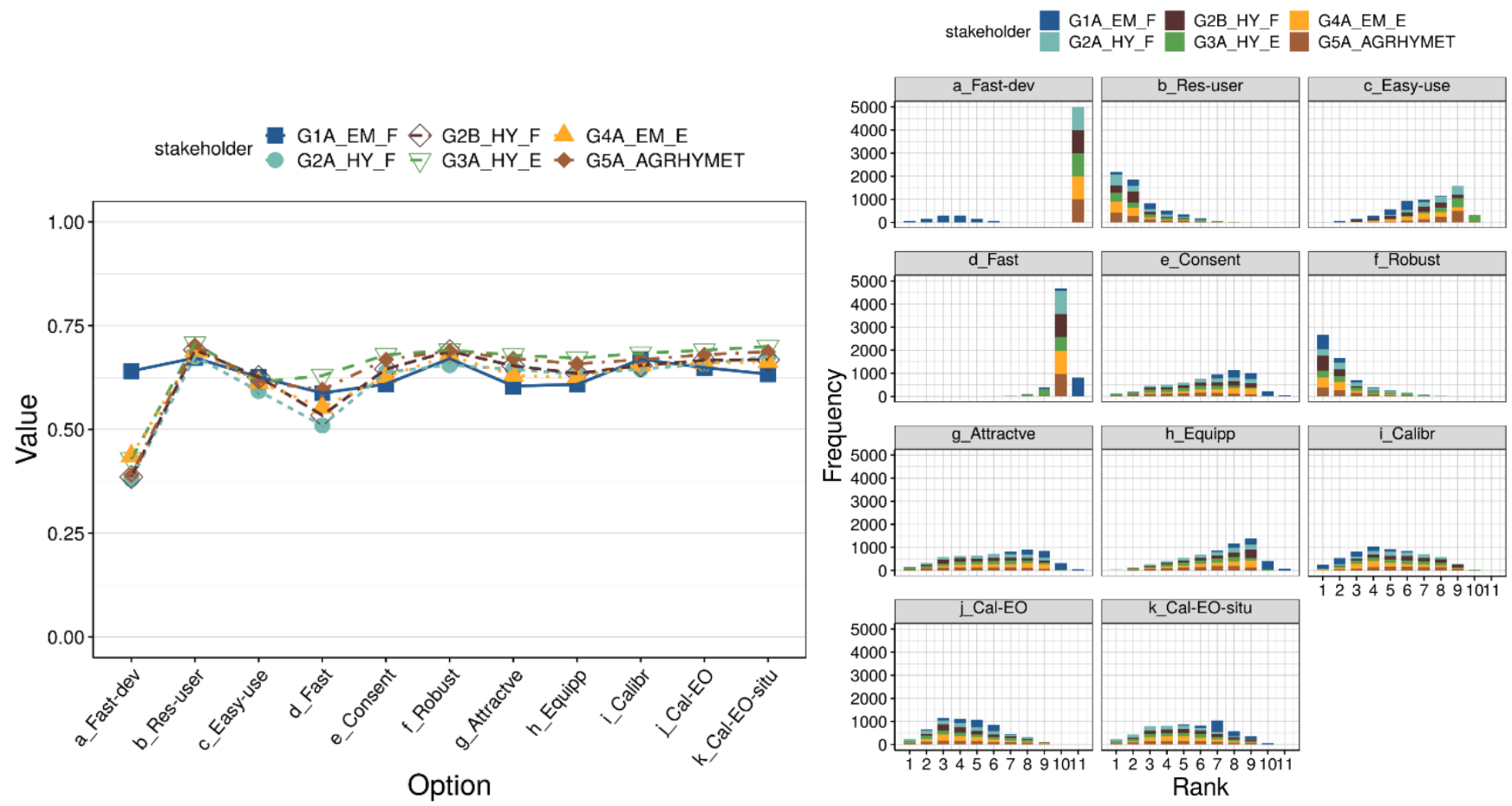

Figure SI-45: Results of the sensitivity analysis setting S31, doubling weight of "Several languages" for all six groups.

\subsection{MCDA results: cost-benefit visualizations}

A cost-benefit visualization using MCDA allows to check, whether configurations are outperformed by other configurations, given the underlying conditions of the MCDA model used. The ValueDecisions app easily allows to visualize such cost-benefit visualizations on chosen attributes with one mouse click. We illustrate three such analyses here. First, we carried out a "classic" cost-benefit visualization, where the total operation and maintenance costs (i.e., attribute 32 _costs) of each FANFAR system configuration were plotted against all other "benefits". In our case, the benefits were the total aggregated value that an configuration achieved for the other nine objectives when aggregating over all nine objectives, using our standard MCDA model (and in including the stakeholder preferences; Figure SI-46). The more to the left the configuration is situated, the more expensive it is (achieving a low value). The configurations with highest performance on both the $\mathrm{x}$ - and y-axis were then linearly connected. This interpolation visualizes a possible efficient frontier for the given configurations, and any configuration below this line is outperformed by one or more other configurations in this set. Note that we cannot show the estimated costs in $€$ as normally done for confidentiality reasons. Obviously, the cheapest configuration (to the far right) was $a_{-} F a s t-d e v$, the status quo configuration at the beginning of the FANFAR project. Not surprisingly, it also achieved the lowest value of all configurations for the other nine objectives. 
Potentially interesting configurations are those situated on the efficient frontier (solid black line), and strictly speaking, configurations below the efficient frontier should not be considered. To give some guidance: FANFAR system configuration $d_{\text {_Fast }}$ (green dot) should definitely not be chosen. It was similarly expensive for operation and maintenance as $c \_$Easy-use, $j_{-}$Cal-EO, and $f \_$Robust, which all achieved higher values for the other nine objectives, for all stakeholder groups. Comparing these four configurations, the best choice is $f_{-}$Robust, which was located on the efficient frontier for all stakeholder groups. Configuration $i_{-}$Calibr could also be a good choice, it was somewhat cheaper, but also achieved a high value on the other objectives for all stakeholder groups. Of the most expensive configurations in terms of operation and maintenance costs were b_Res-user, e_Consent, g_Attractve, $h$ Equipp, and k-Cal-EO-situ. For these, it is difficult to decide which is best, because of differences between stakeholder groups. For instance, $g \_$Attractve achieved high values on the nine other objectives for stakeholder groups G2A, G2B, G3A, and G5A, but was outperformed by configuration $b \_$Res-user and $f \_$Robust for group G4A, and clearly performed poorly for group G1A. For this group of the French speaking emergency managers, G1A, $b \_$Res-user would be the best performing of the expensive configurations.

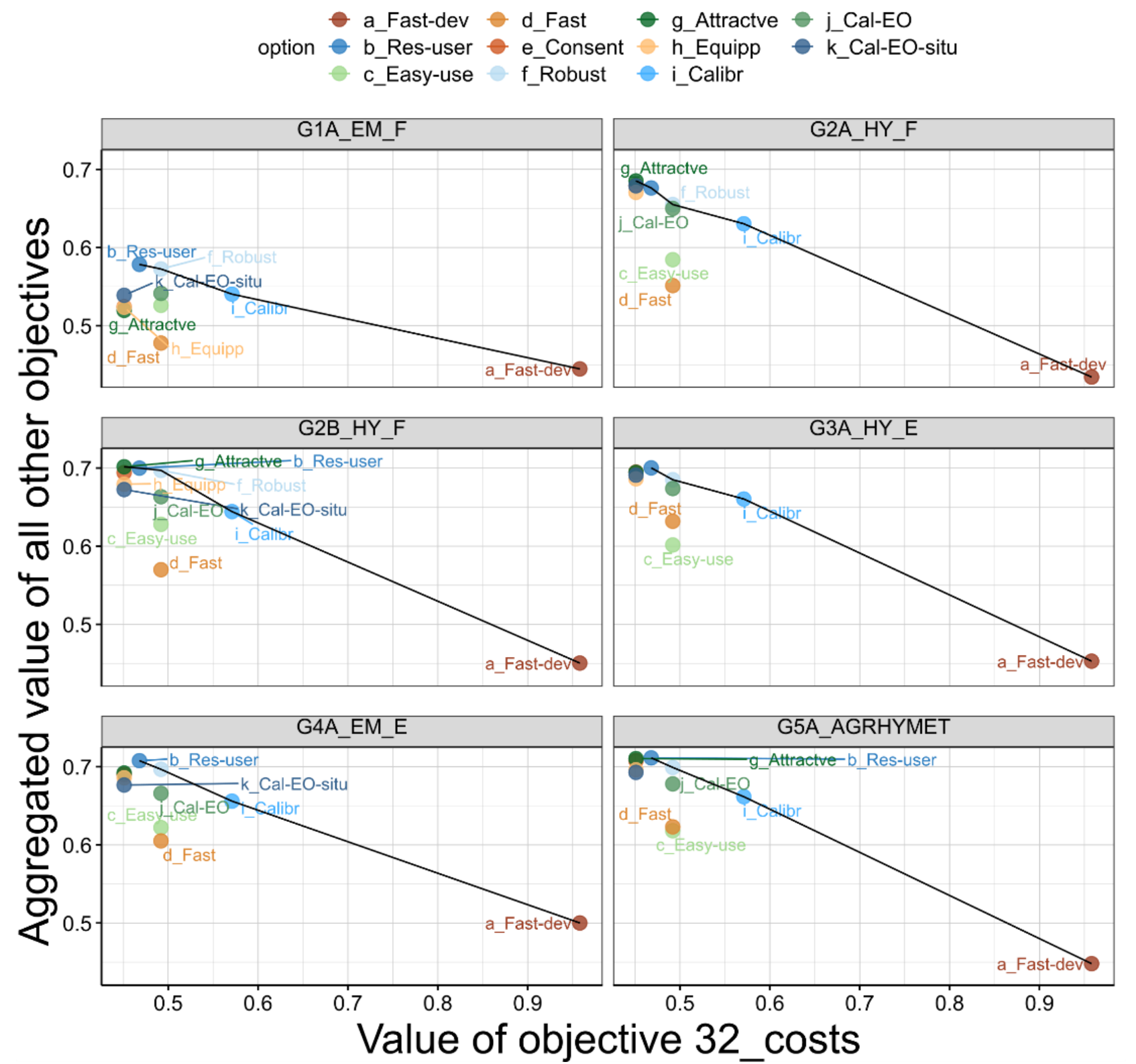

Figure SI-46. Cost-benefit visualization of 11 FANFAR system configurations (colored dots) for six stakeholder groups (blocks). The value of the total operation and maintenance costs (i.e., attribute 32_costs; $x$-axis) is plotted against the total aggregated value of all other nine objectives (y-axis). For both axes, values range from 0 (worst case) to 1 (best). This means that the most expensive configurations are to the left (i.e., achieving low values), and the cheaper configurations to the right (achieving high values). The efficient 
frontier (solid black line) visualizes the best performing configurations for a given cost level. Configurations situated below the efficient frontier are outperformed by better configurations, which achieve a higher performance on the other nine objectives for same level of operation and maintenance costs.

We repeated the cost-benefit visualization for both lower level objectives of the objective 3 Low costs, i.e., 3.1 Short development time, and 3.2 Low operation and maintenance costs (Figure SI-47) as costs, plotted against the total aggregated value of all other eight (respectively seven) objectives. Interestingly, configuration $d_{-}$Fast now appeared on the efficient frontier for four stakeholder groups, while it had been outperformed by other configurations in the previous analysis. Reason is that $d$-Fast is not only the fastest system for producing and distributing forecasts, but because it has no fancy features, it is also the fastest configuration in terms of development time (apart from the status quo configuration $a \_$Fast-dev). Thus, $d$-Fast performed quite well for those stakeholder groups who had given a relatively higher weight to the objective 31_develop_time, namely groups G2A, G2B, G4A, and G5A. Previously recommendable configurations remained on the efficient frontier for all stakeholder groups: $b \_$Res-user, f_Robust, and $i_{-}$Calibr.
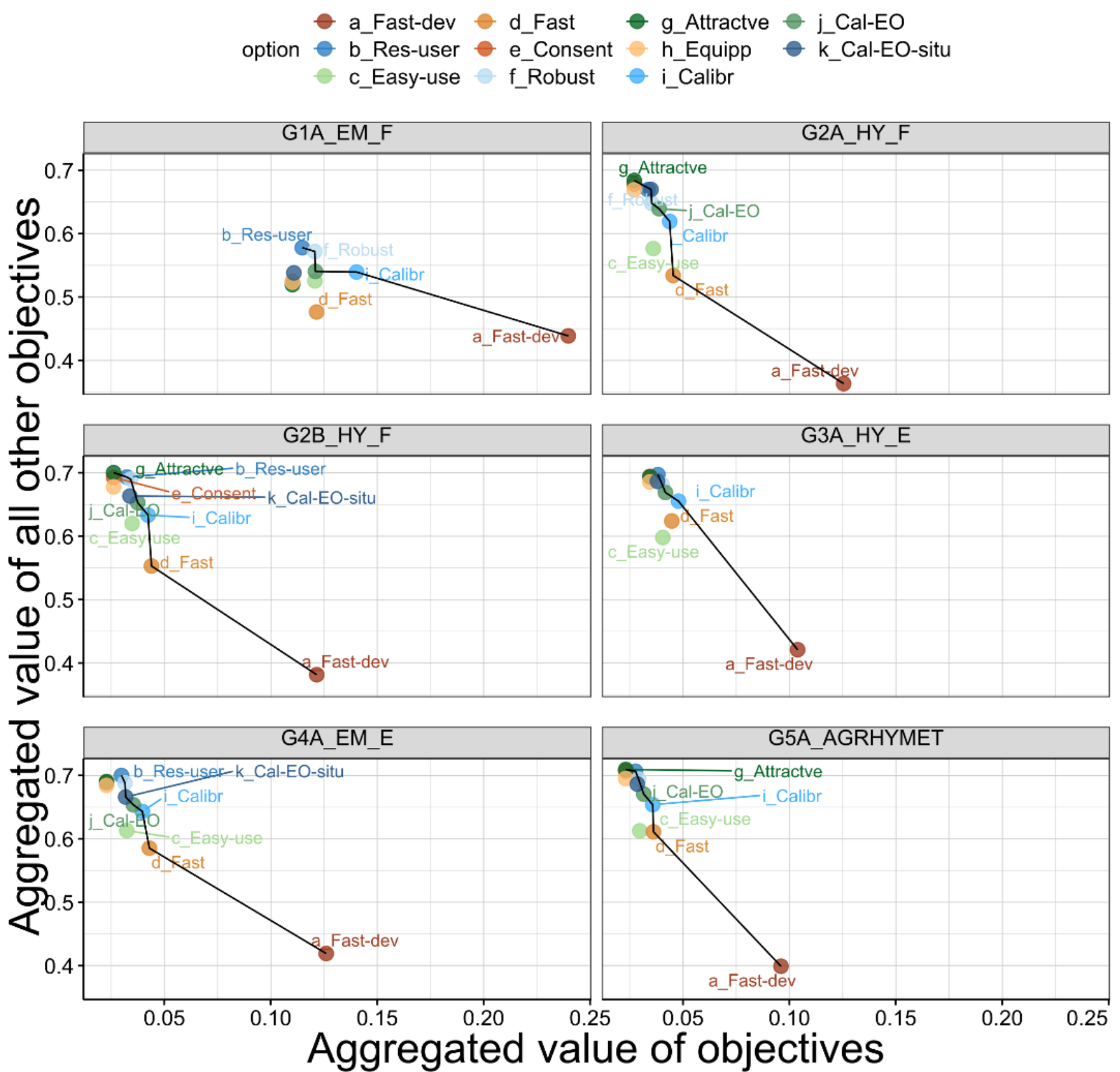

Aggregated value of objectives

32_costs and 31_develop_time 
Figure SI-47. Cost-benefit visualization of 11 FANFAR system configurations (colored dots) for six stakeholder groups (blocks). The costs ( $x$-axis) are the aggregated value of the total operation and maintenance costs (i.e., attribute 32_costs) and the time needed to develop the FANFAR system (31_develop_time). Costs are plotted against the total aggregated value of all other eight objectives ( $y$ axis). Solid black line: efficient frontier.

In the third cost-benefit visualization, we used the two previous cost attributes and included another attribute that concerns costs, namely whether long-term financing is secured (41_sust_financing; Figure SI-48). Thus, the "costs" displayed on the xaxis are the value achieved when aggregating over all three objectives, using our standard MCDA model (and including the stakeholder preferences). Of the previously recommendable configurations $b \_$Res-user, and $f \_$Robust are still (nearly) on the efficient frontier for all stakeholder groups. However, for groups G2A, G2B, and G5A, $i_{-}$Calibr were now outperformed. Configuration $g \_$Attractve performed well for five stakeholder groups, but - again - not for the French speaking emergency managers, group G1A. This can be explained in that they had given zero weight, i.e., zero importance to 41_sust_financing. Thus, if we are looking for a consensus configuration over all groups, $g \_$Attractve should not be chosen.
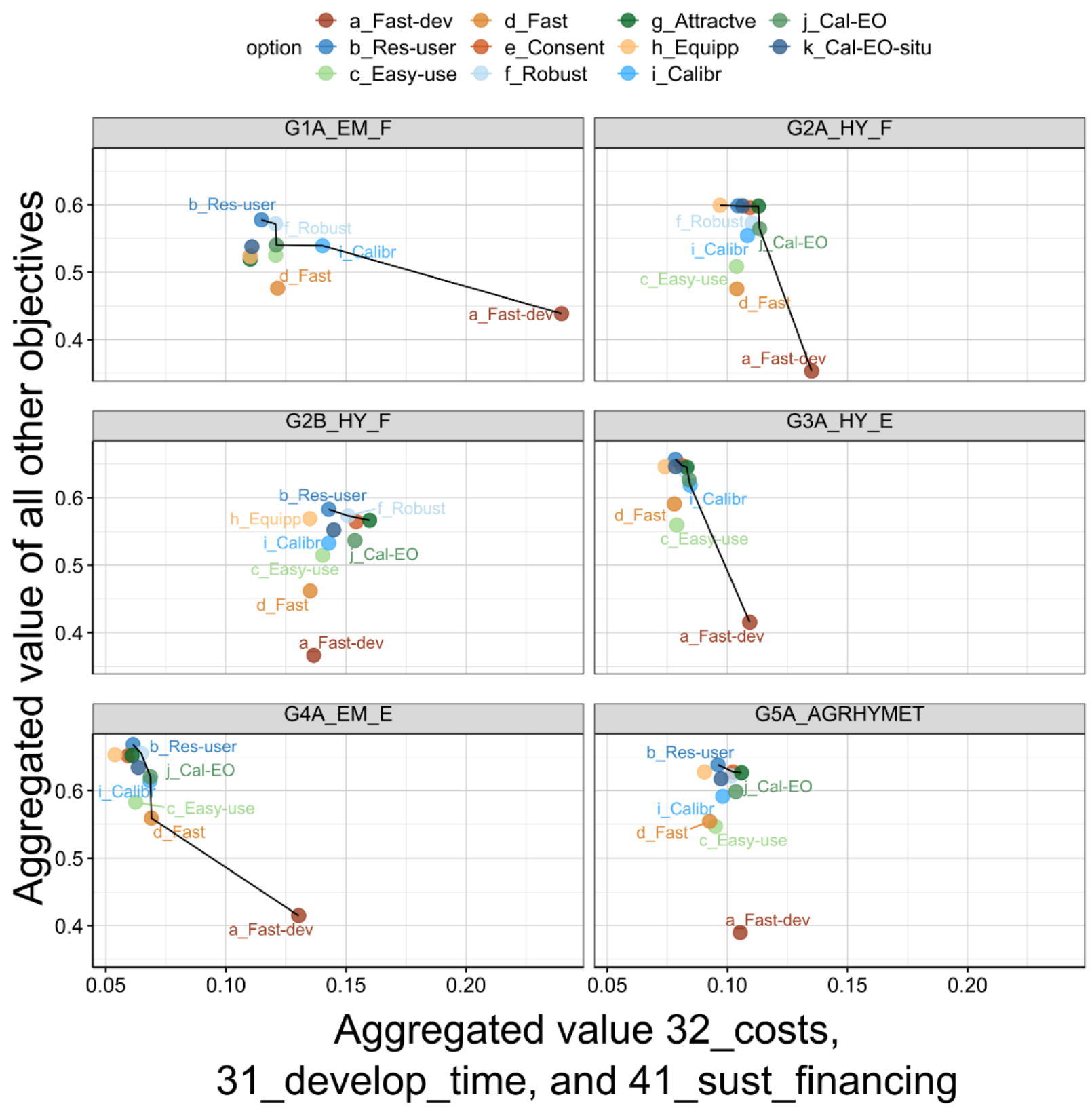

Figure SI-48. Cost-benefit visualization of 11 FANFAR system configurations (colored dots) for six stakeholder groups (blocks). The costs (x-axis) are the aggregated value of the total operation and maintenance costs (i.e., attribute 32_costs), the time needed to develop the FANFAR system (31_develop_time), and secured long-term financing (41_sust_financing). Costs are plotted against the total aggregated value of all other eight objectives ( $y$-axis). Solid black line: efficient frontier. 


\subsection{Results: stakeholders' perceived satisfaction with FANFAR system}

12 workshop participants filled out the survey, resulting for our 10 objectives in $10 \times 12=120$ responses to each question (Figure SI-49). Both from the directly asked question, whether respondents would use the FANFAR system in future if it remains as is (b), as well as the inferred difference between the acceptable level (c) and the perceived current level (a) of each objective $(c-a)$, the majority of respondents seemed to perceive FANFAR's current performance as sufficient for all objectives. Across all objectives, 79 responses were positive, 16 negative, and 25 did not respond to question $b$ (Figure SI-49). Regarding the difference $(c-a), 97$ responses indicated higher perceived current performance (a) than the minimum required performance (c), while 23 responses were opposite (Figure SI-49). However, although this inferred measure of sufficiency of the FANFAR system performance and question $b$ should measure the same opinion, only in four cases did negative responses to $\mathrm{b}$ correspond to inferred negative responses. This indicates that respondents were not always consistent in their answers. Differences between performances of our ten objectives were moderate. Objective 42_human_resour performed best with only two (out of 21, as three NA) combined negative responses to b and ( $\mathrm{c}-\mathrm{a}$ ). Objectives 21_reliable_info and 22_timely_info performed worst, with each six combined negative responses (out of 22, as two NA).

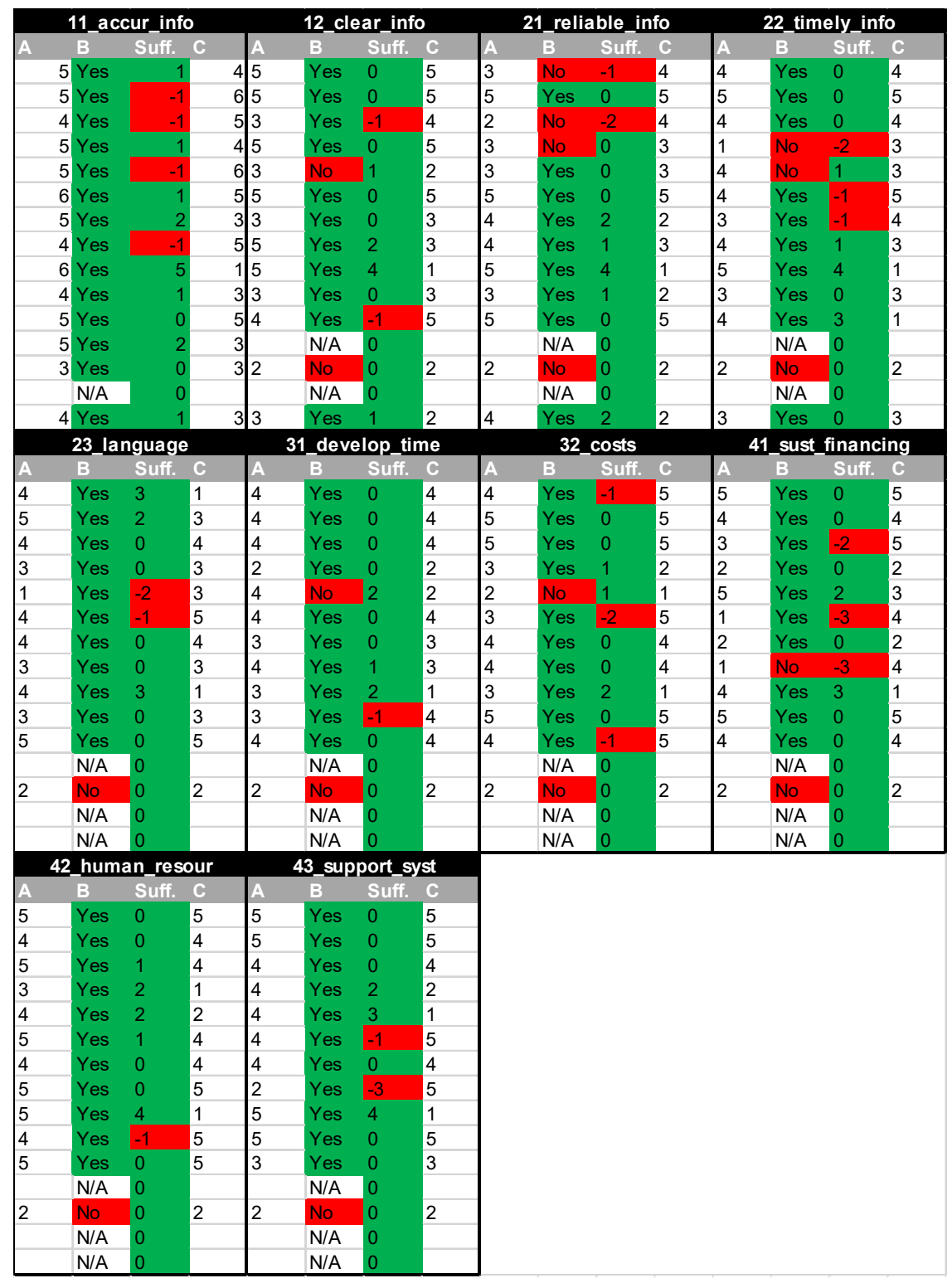

Figure SI-49. Stakeholder perceived satisfaction with the performance of the FANFAR system during the 2020 rainy season. Survey results for each objective and survey respondent $(\mathrm{N}=12)$. $A, B$ and $C$ refer to the three questions asked for each objective. Answers are represented by descending numbers from "best" to "worst" performance. 'Suff.' represents the calculated difference between the answer of $\mathrm{C}$ and $\mathrm{A}$. Green colored cells represent a positive response (respondent indicating their perceived performance is sufficient for use of FANFAR), while red indicates the opposite. 


\section{References}

Andersson, J. C. M., Arheimer, B., Traoré, F., Gustafsson, D., and Ali, A.: Process refinements improve a hydrological model concept applied to the Niger River basin, Hydrological Processes, 31, 4540-4554, https://doi.org/10.1002/hyp.11376, 2017.

Arheimer, B., Pimentel, R., Isberg, K., Crochemore, L., Andersson, J. C. M., Hasan, A., and Pineda, L.: Global catchment modelling using World-Wide HYPE (WWH), open data, and stepwise parameter estimation, Hydrol. Earth Syst. Sci., 24, 535559, 10.5194/hess-24-535-2020, 2020.

Berg, P., Donnelly, C., and Gustafsson, D.: Near-real-time adjusted reanalysis forcing data for hydrology, Hydrol. Earth Syst. Sci., 22, 989-1000, 10.5194/hess-22-989-2018, 2018.

Berg, P., Almén, F., and Bozhinova, D.: HydroGFD3.0: a $25 \mathrm{~km}$ global near real-time updated precipitation and temperature data set, Earth Syst. Sci. Data Discuss., 2020, 1-18, 10.5194/essd-2020-236, 2020.

Eisenführ, F., Weber, M., and Langer, T.: Rational Decision Making, Springer, Berlin, Heidelberg, New York, 2010.

Figueira, J., and Roy, B.: Determining the weights of criteria in the ELECTRE type methods with a revised Simos' procedure, European Journal of Operational Research, 139, 317-326, https://doi.org/10.1016/S0377-2217(01)00370-8, 2002.

Gregory, R., Long, G., Colligan, M., Geiger, J. G., and Laser, M.: When experts disagree (and better science won't help much): Using structured deliberations to support endangered species recovery planning, Journal of Environmental Management, 105, 30-43, 10.1016/j.jenvman.2012.03.001, 2012.

Gupta, H. V., Kling, H., Yilmaz, K. K., and Martinez, G. F.: Decomposition of the mean squared error and NSE performance criteria: Implications for improving hydrological modelling, J. Hydrol., 377, 80-91, 10.1016/j.jhydrol.2009.08.003, 2009.

Haag, F., Lienert, J., Schuwirth, N., and Reichert, P.: Identifying non-additive multi-attribute value functions based on uncertain indifference statements, Omega-International Journal of Management Science, 85, 49-67, 10.1016/j.omega.2018.05.011, 2019.

Haag, F., Aubert, A. H., and Lienert, J.: Supporting environmental decisions with multiple objectives, multiple stakeholders, and uncertainty with the ValueDecisions web app, Under review, subm.

Howard, R. A.: Decision-analysis - Practice and promise, Manage. Sci., 34, 679-695, 10.1287/mnsc.34.6.679, 1988.

Kendall, M. G.: A new measure of rank correlation, Biometrika, 30, 81-93, 10.2307/2332226, 1938.

Litcanu, M., Prostean, O., Oros, C., and Mnerie, A. V.: Brain-writing vs. brainstorming case study for power engineering education, in: Proceedings of 6th World Conference on Educational Sciences, edited by: Uzunboylu, H., Procedia Social and Behavioral Sciences, 387-390, 2015.

Marttunen, M., Belton, V., and Lienert, J.: Are objectives hierarchy related biases observed in practice? A meta-analysis of environmental and energy applications of Multi-Criteria Decision Analysis, European Journal of Operational Research, 265, 178-194, 10.1016/j.ejor.2017.02.038, 2018.

Paulus, P. B., and Yang, H. C.: Idea generation in groups: A basis for creativity in organizations, Organizational Behavior and Human Decision Processes, 82, 76-87, 10.1006/obhd.2000.2888, 2000.

Pictet, J., and Bollinger, D.: Extended use of the cards procedure as a simple elicitation technique for MAVT. Application to public procurement in Switzerland, European Journal of Operational Research, 185, 1300-1307, 10.1016/j.ejor.2006.05.051, 2008.

Silva Pinto, F., and Lienert, J.: Deliverable: D2.1. Report activities to establish co-design committee, stakeholder analysis, European Union, Horizon 2020, Innovation Action ICT programme; Project: 780118 FANFAR, Deliverable D2.1, $48,2018$. Zheng, J., Egger, C., and Lienert, J.: A scenario-based MCDA framework for wastewater infrastructure planning under uncertainty, Journal of Environmental Management, 183, Part 3, 895-908, http://dx.doi.org/10.1016/j.jenvman.2016.09.027, 2016. 